Supporting Information for

\title{
Expansion of Phosphane Treasure Box for Staudinger Peptide Ligation
}

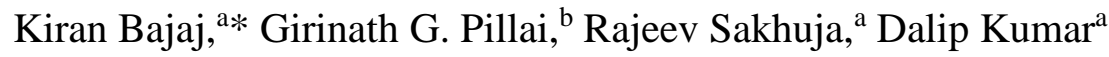

a Department of Chemistry, Birla Institute of Technology and Science, Pilani 333031, Rajasthan, India

${ }^{\mathrm{b}}$ Chemistry Division, Nyro Research India, Kochi 682021, Kerala, India

E-mail: kbajaj26@gmail.com

Contents:

1. Original NMR of 2

S02

2. Original NMR and HRMS spectra of $\mathbf{4 a}-\mathbf{4} \mathbf{i}, \mathbf{4} \mathbf{i}^{\prime}$

S03-S21

3. Original NMR spectra of $\mathbf{5 a - 5 i}$

S21-S33

4. Original NMR and HRMS spectra of $\mathbf{6 a a}-\mathbf{6 i} \mathbf{h} \mathbf{h}$

S34-S58

5. Comparison of ${ }^{1} \mathrm{H}$ NMR of Cbz-L-Ala-L-Ala- $p$-tol (6bc) with Cbz-L-Ala-DL-Ala- $p$-tol S59

6. Computational data: coordinates, imaginary frequency and energy data. S60-S91 
1. $\quad{ }^{1} \mathrm{H}$ NMR (400 MHz, Chloroform- $d$ ) spectra of compound 2

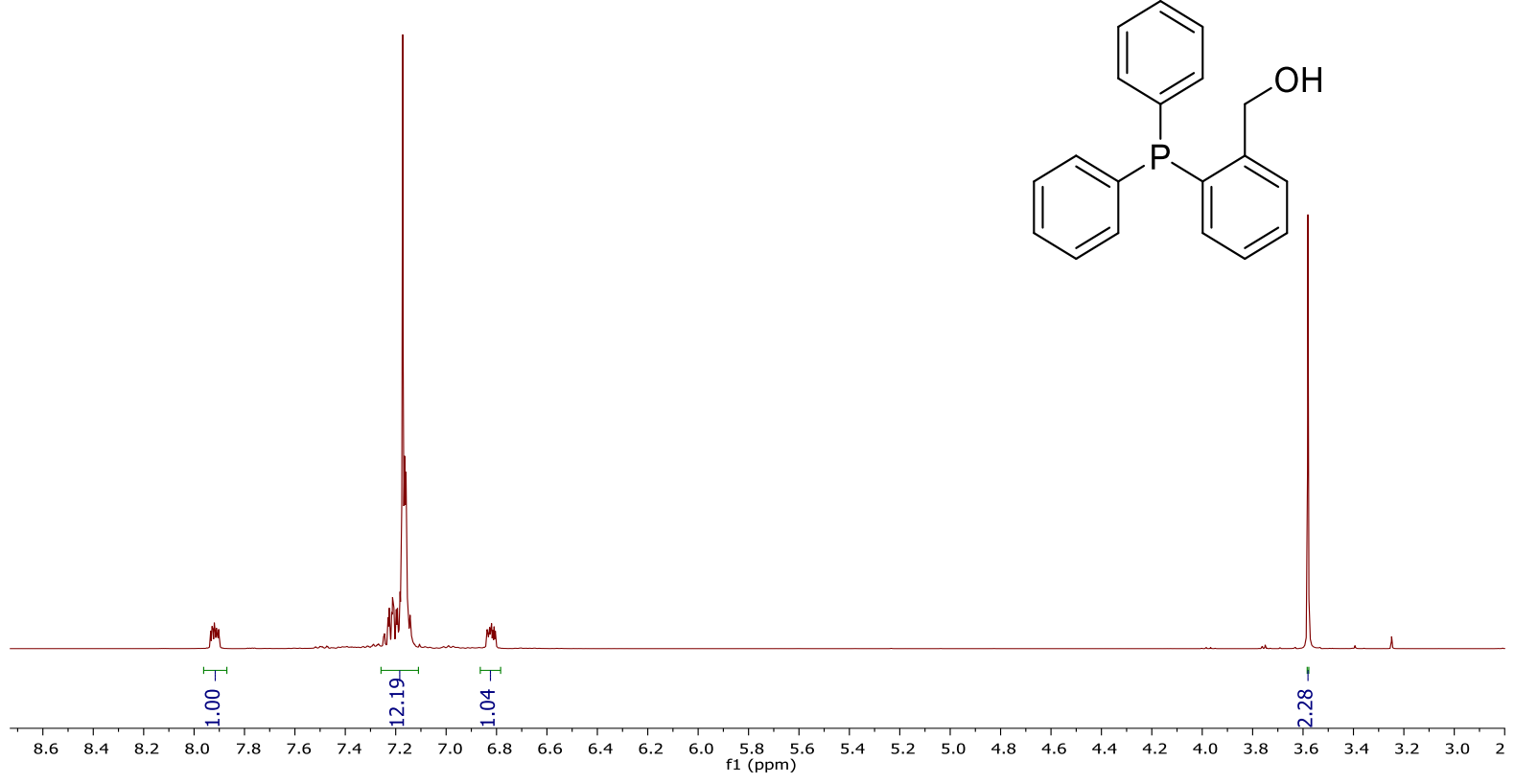

${ }^{31} \mathrm{P}\left\{{ }^{1} \mathrm{H}\right\}$ NMR (162 MHz, Chloroform- $d$ ) spectra of compound 2

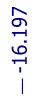<smiles>OCc1ccccc1P(c1ccccc1)c1ccccc1</smiles> 
2. Original NMR and HRMS spectra of $\mathbf{4 a - 4 i}, \mathbf{4} \mathbf{i}^{\prime}$

${ }^{1} \mathrm{H}$ NMR (400 MHz, Chloroform- $d$ ) spectra of $\mathbf{4 a}$

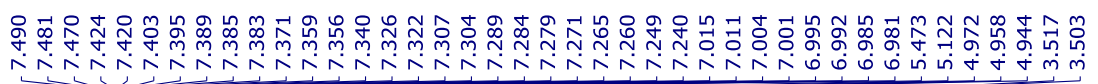<smiles>O=C(CNC(=O)OCc1ccccc1)OCc1ccccc1P(c1ccccc1)c1ccccc1</smiles>

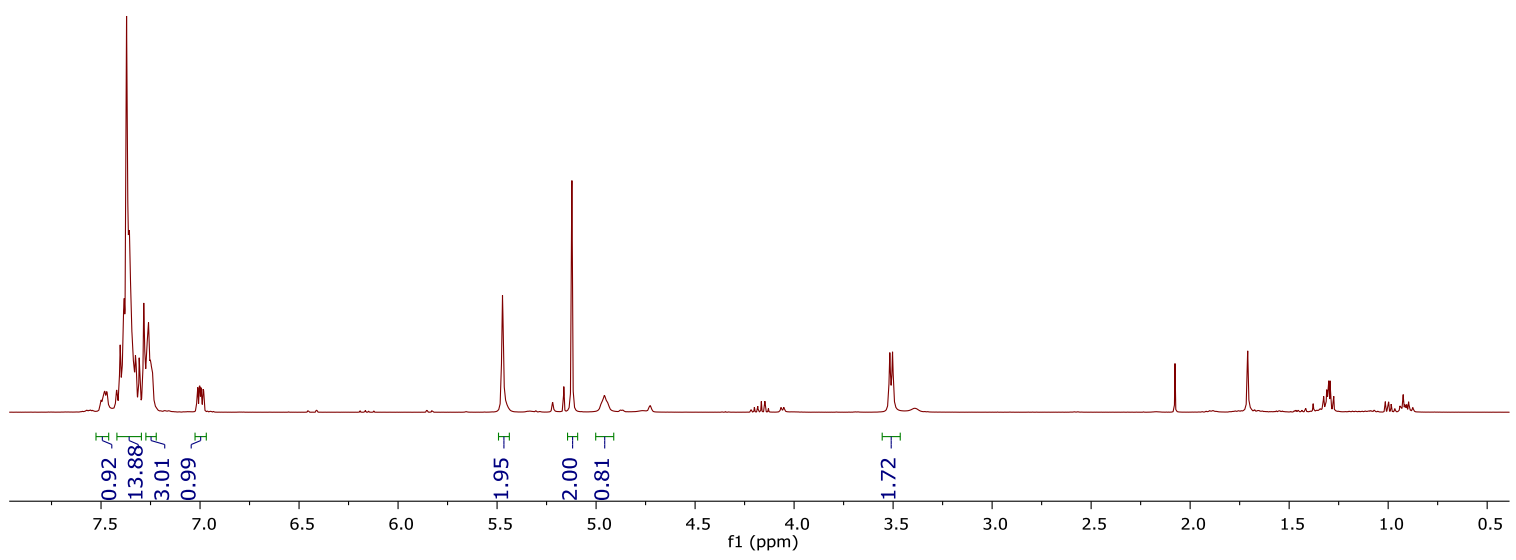

${ }^{13} \mathrm{C}\left\{{ }^{1} \mathrm{H}\right\}$ NMR (101 MHz, Chloroform- $d$ ) spectra of $4 \mathbf{a}$

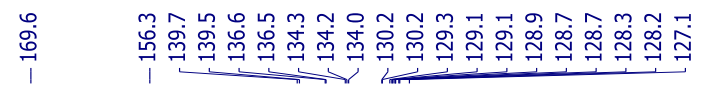

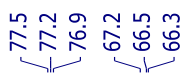

$\stackrel{\stackrel{2}{\dddot{q}}}{1}$
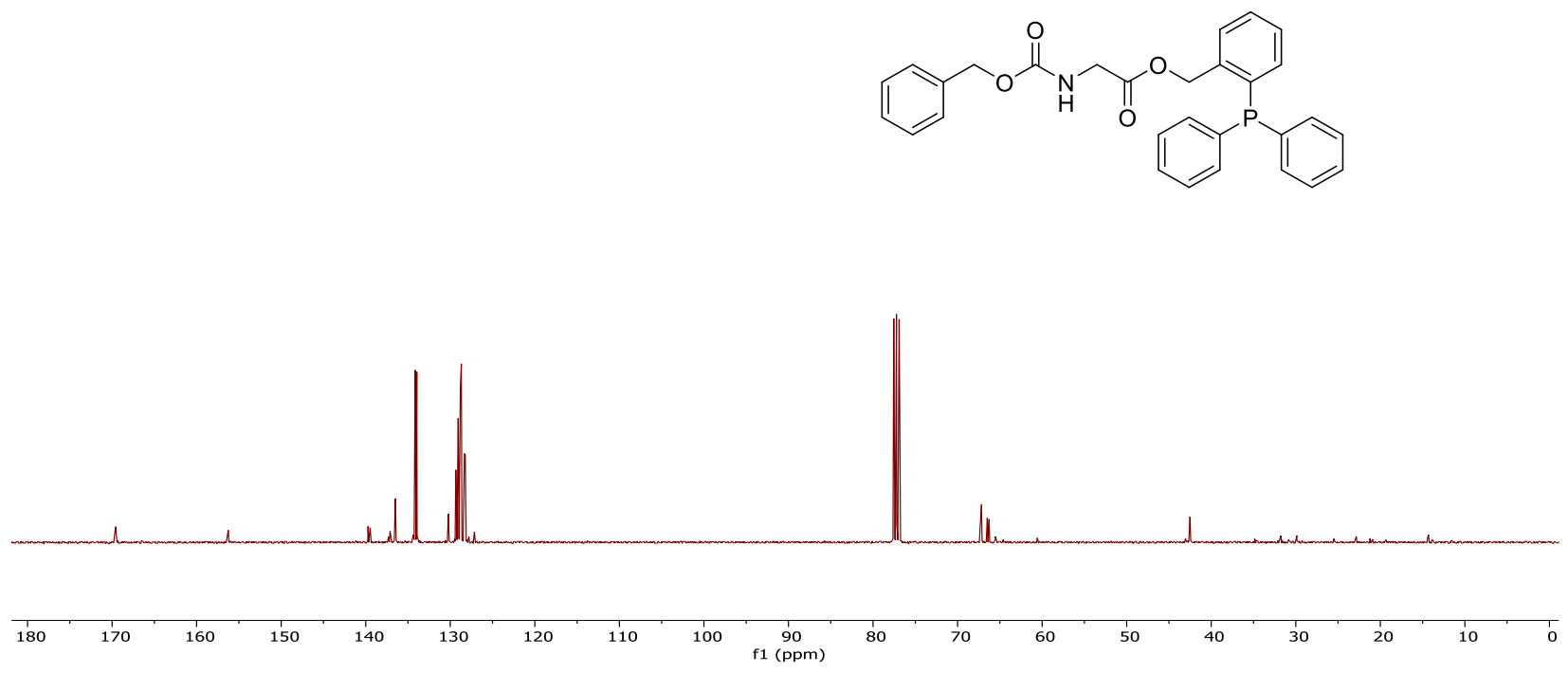

SB 
${ }^{31} \mathrm{P}\left\{{ }^{1} \mathrm{H}\right\}$ NMR (162 MHz, Chloroform- $d$ ) spectra of $\mathbf{4 a}$

9
7
0
1
1
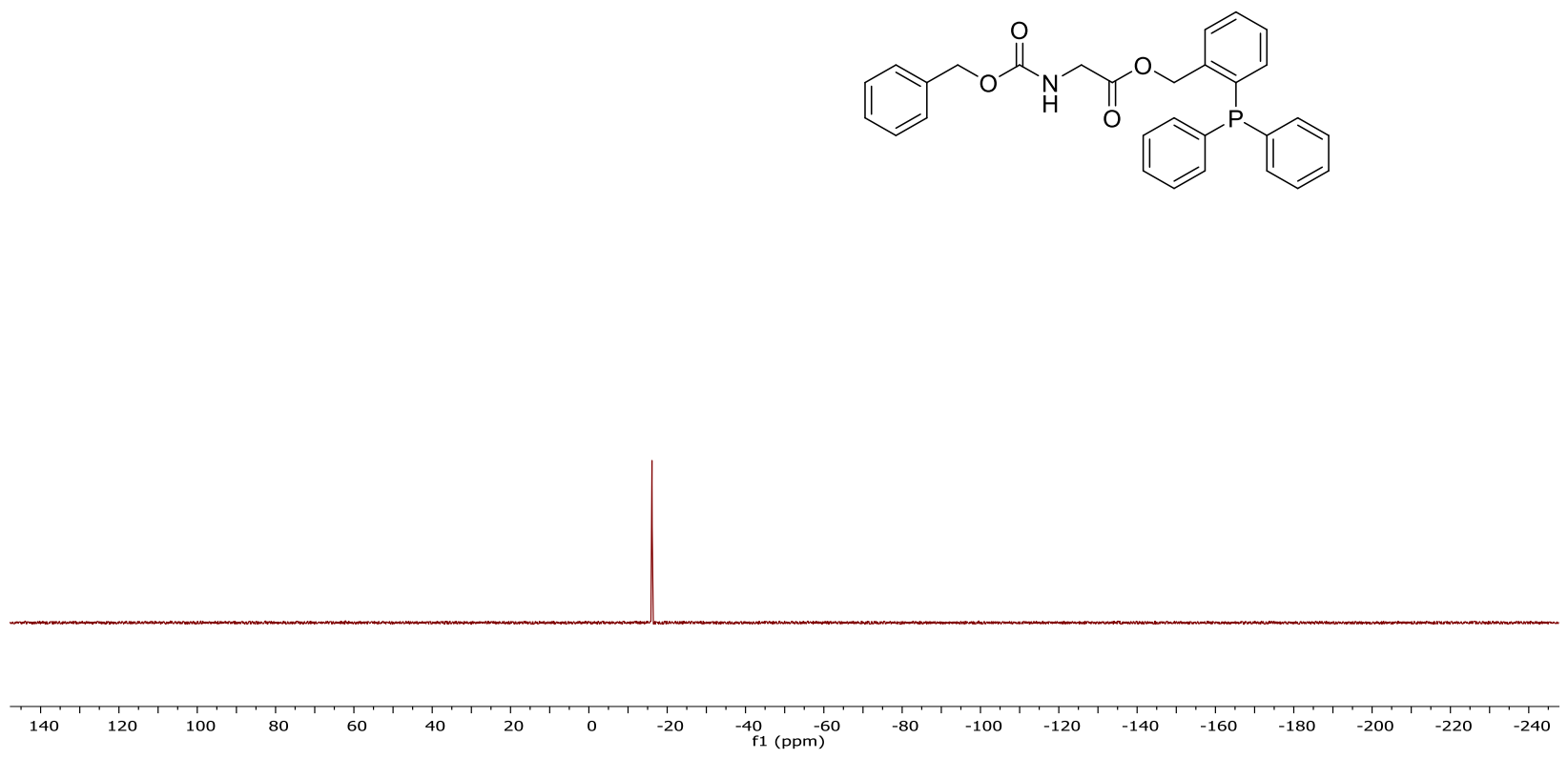

HRMS of $\mathbf{4 a}$

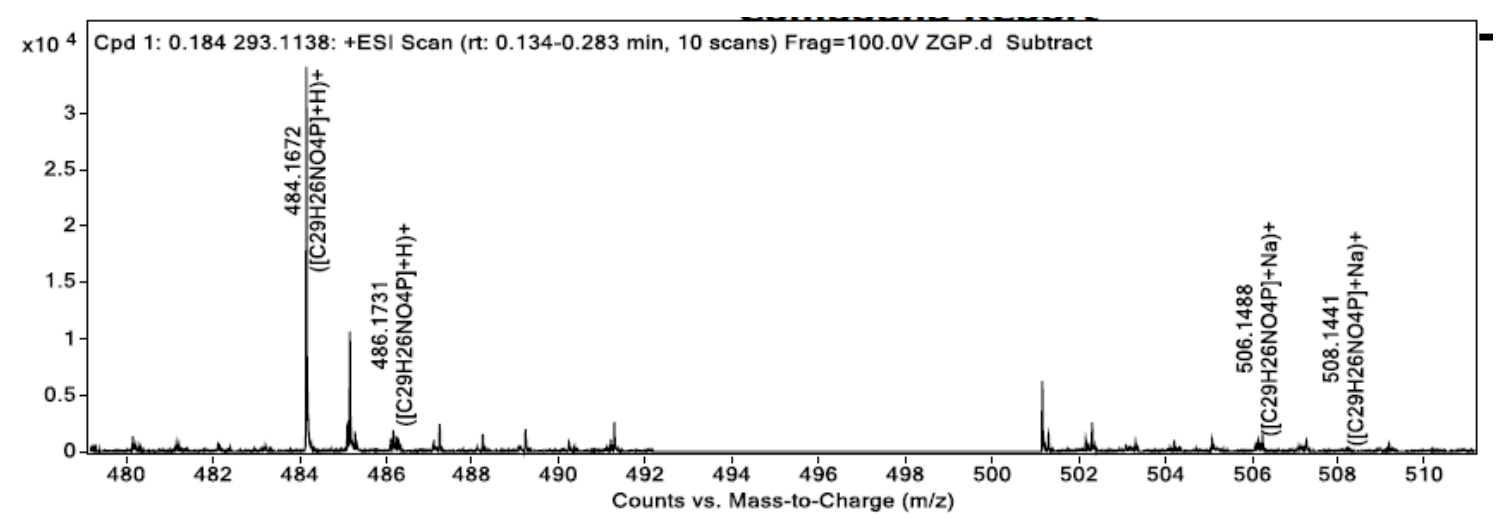



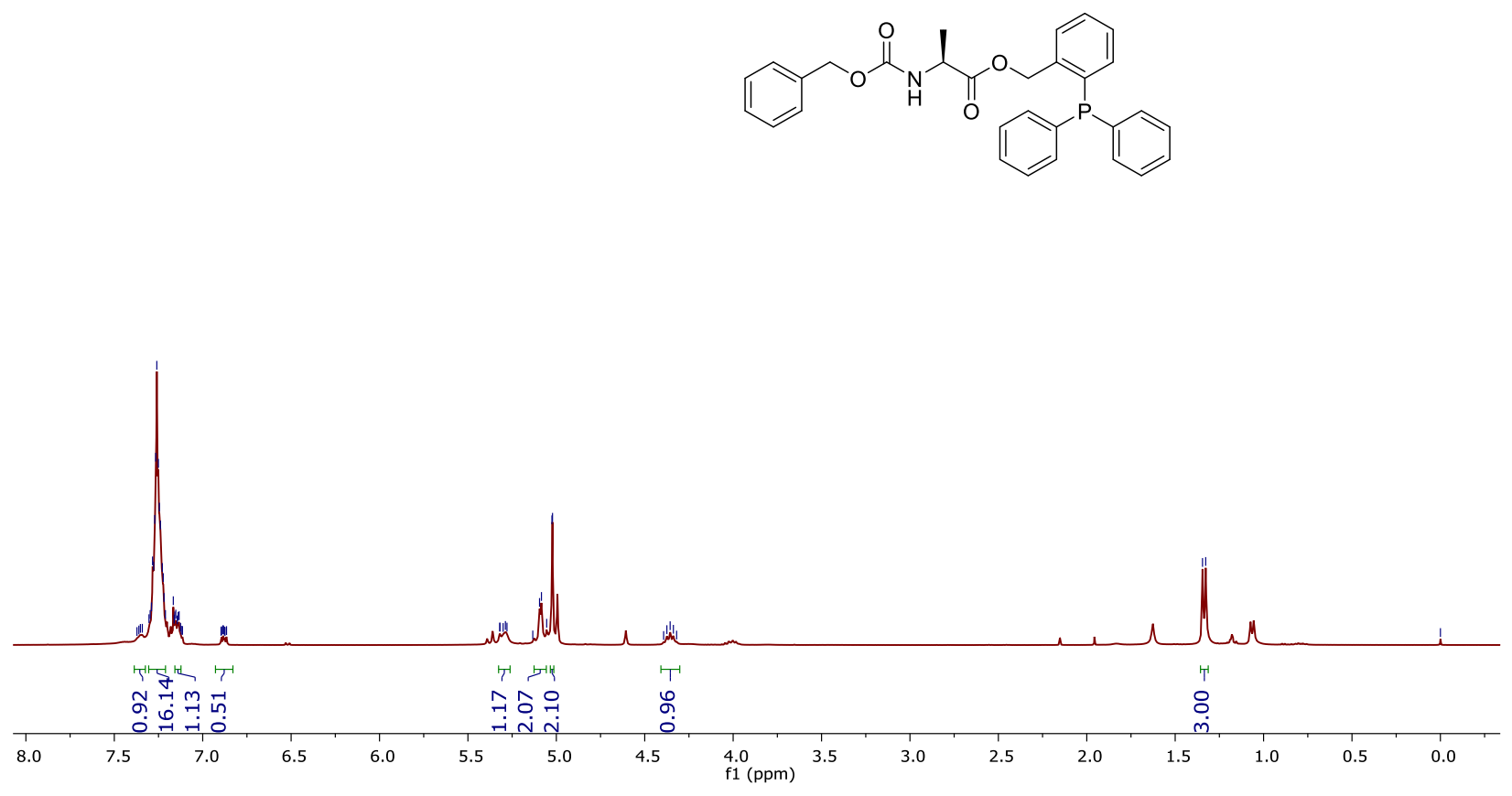

${ }^{13} \mathrm{C}\left\{{ }^{1} \mathrm{H}\right\}$ NMR (101 MHz, Chloroform- $d$ ) spectra of $\mathbf{4 b}$
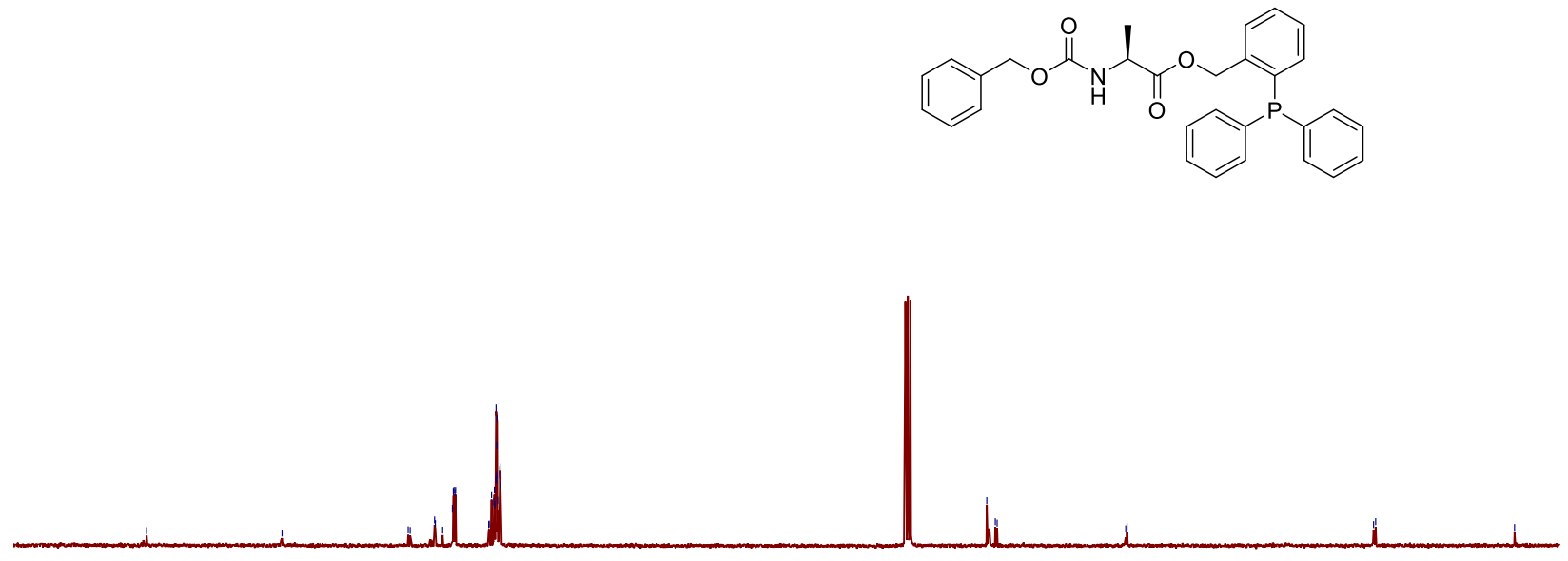
${ }^{31} \mathrm{P}\left\{{ }^{1} \mathrm{H}\right\}$ NMR (162 MHz, Chloroform- $d$ ) spectra of $\mathbf{4 b}$

㝏
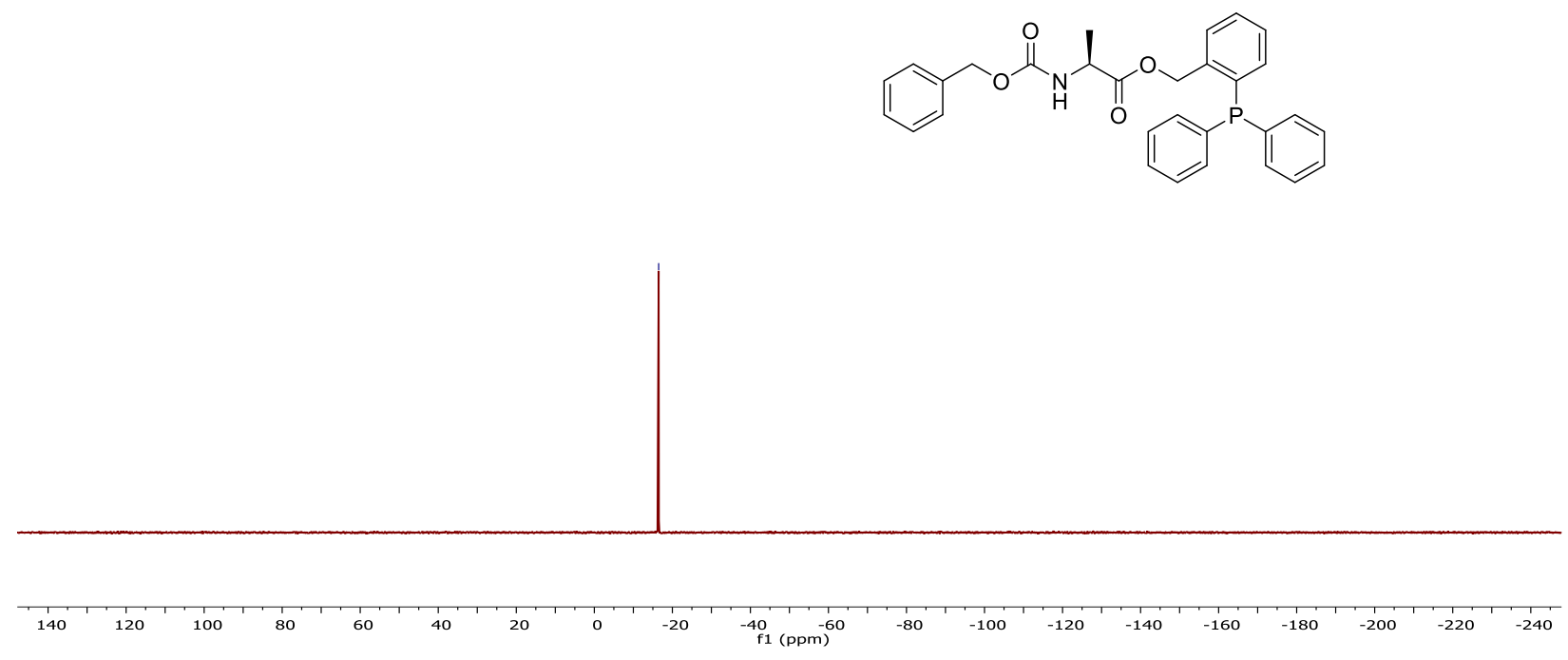

HRMS of $\mathbf{4 b}$

MS Zoomed Spectrum

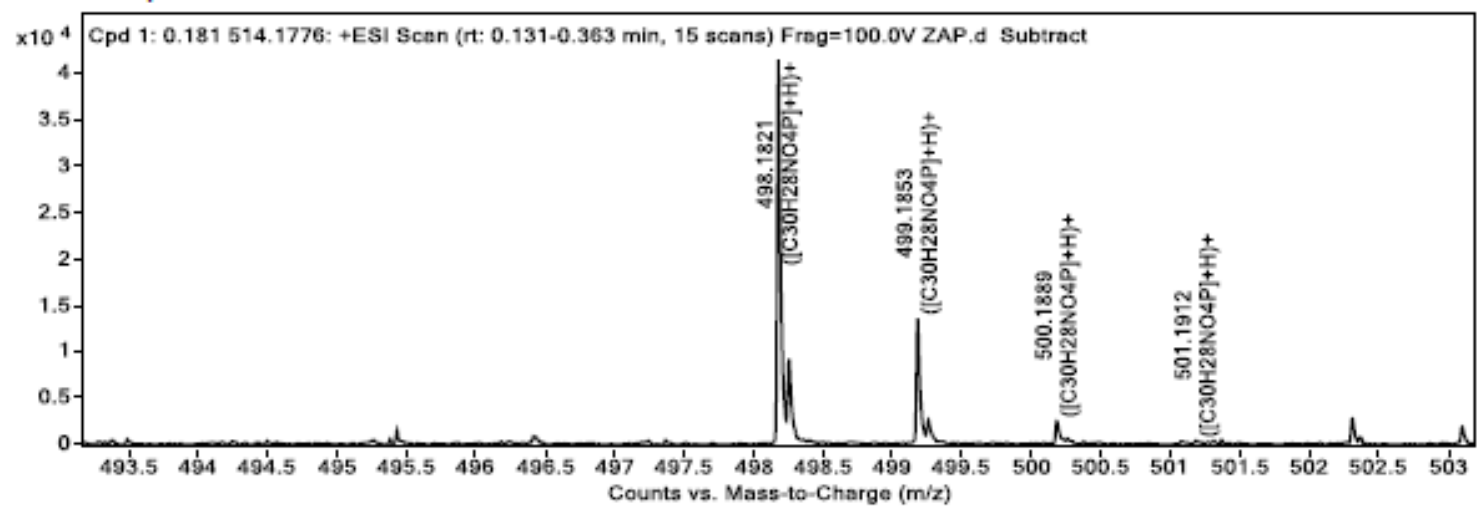


${ }^{1} \mathrm{H}$ NMR (400 MHz, Chloroform- $d$ ) spectra of $\mathbf{4 c}$

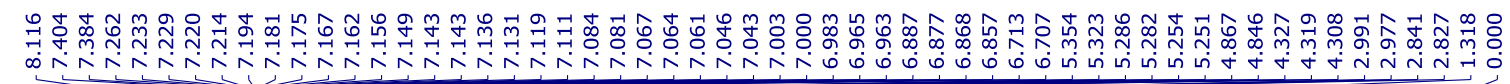

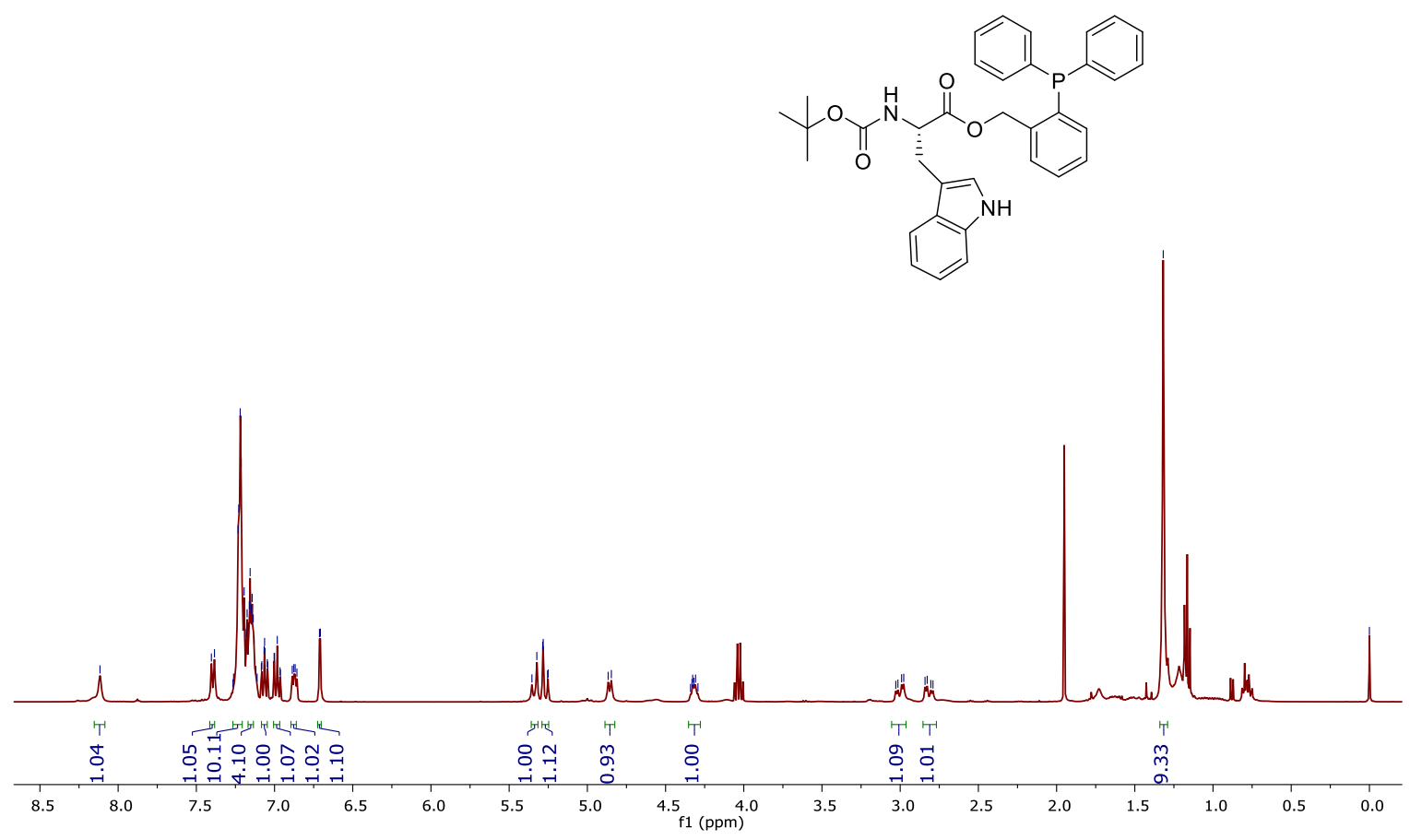

${ }^{13} \mathrm{C}\left\{{ }^{1} \mathrm{H}\right\}$ NMR (101 MHz, Chloroform- $d$ ) spectra of $\mathbf{4 c}$

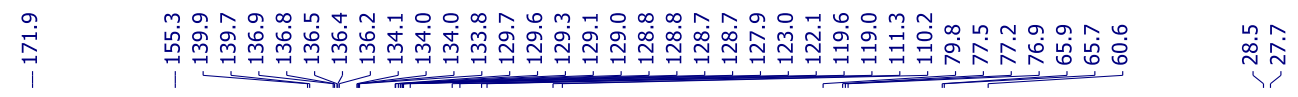
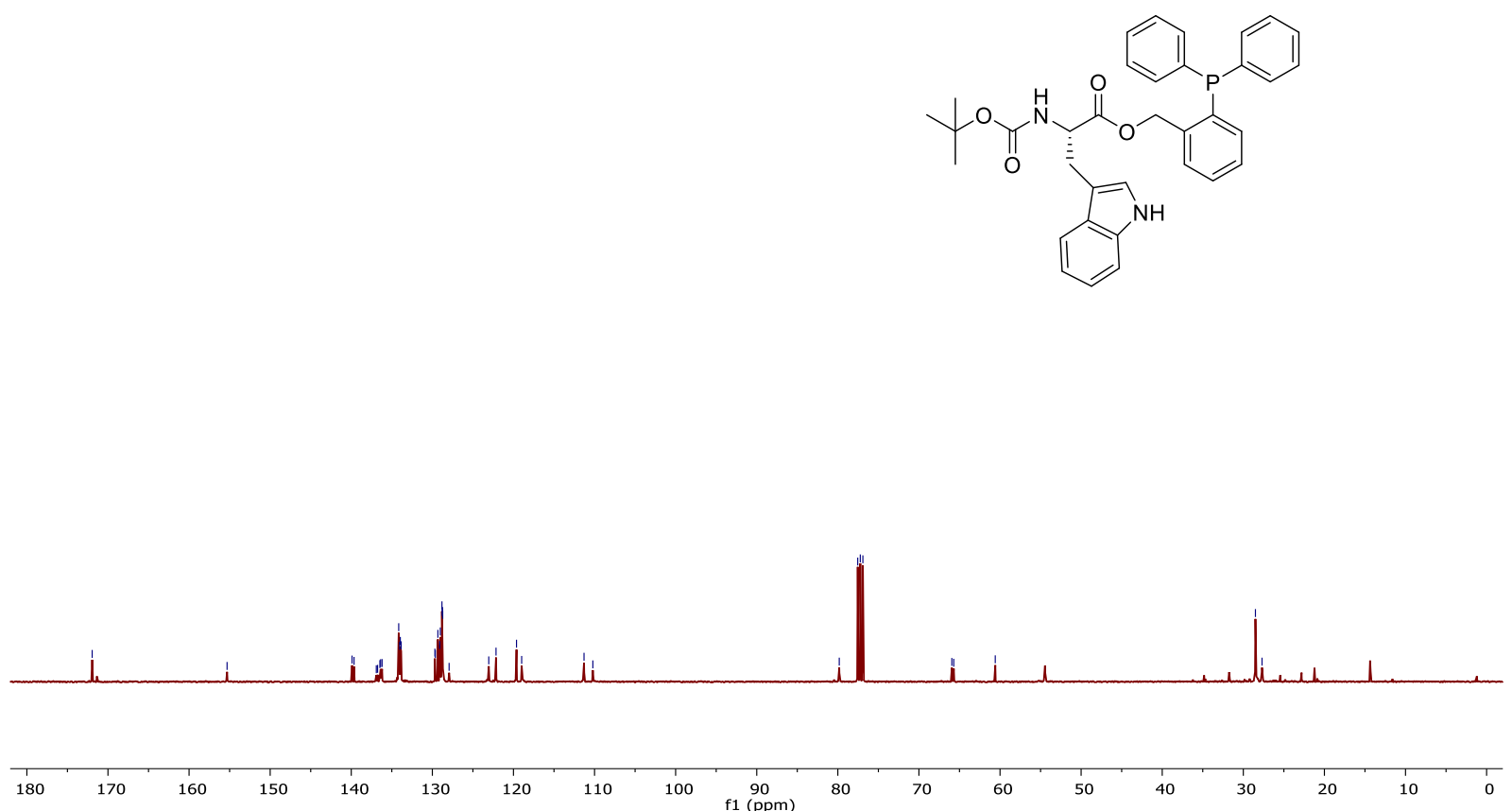


\section{${ }^{31} \mathrm{P}\left\{{ }^{1} \mathrm{H}\right\}$ NMR (162 MHz, Chloroform- $d$ ) spectra of $4 \mathbf{c}$}

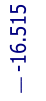
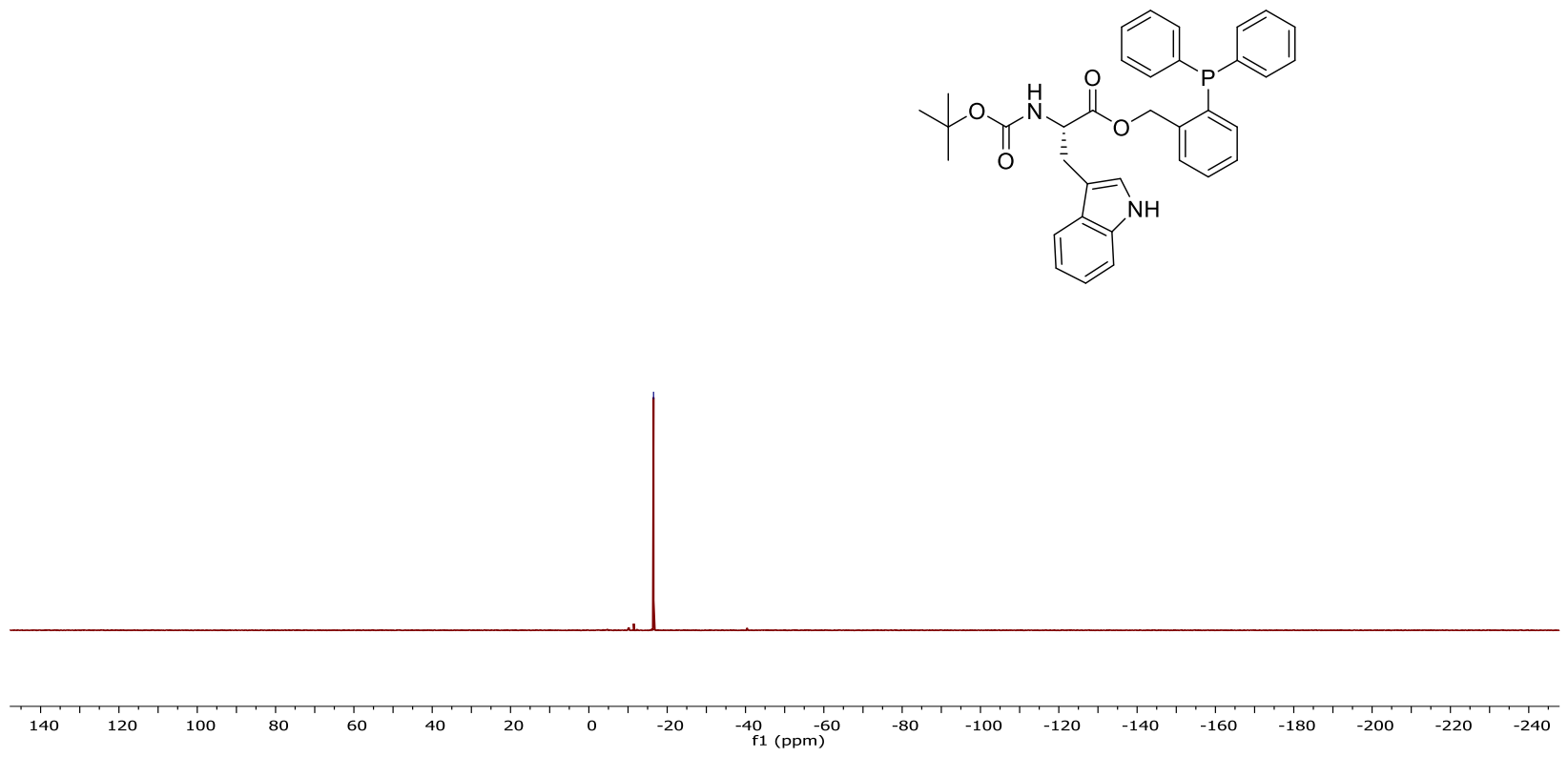

\section{HRMS of $\mathbf{4 c}$}

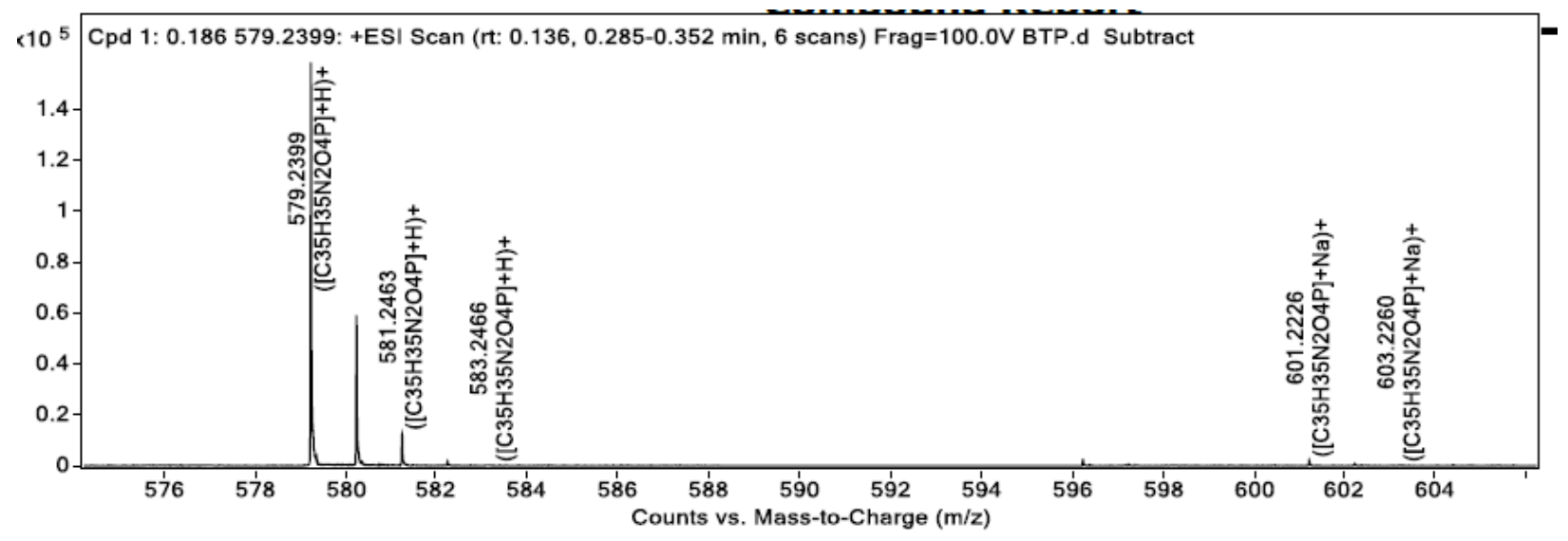




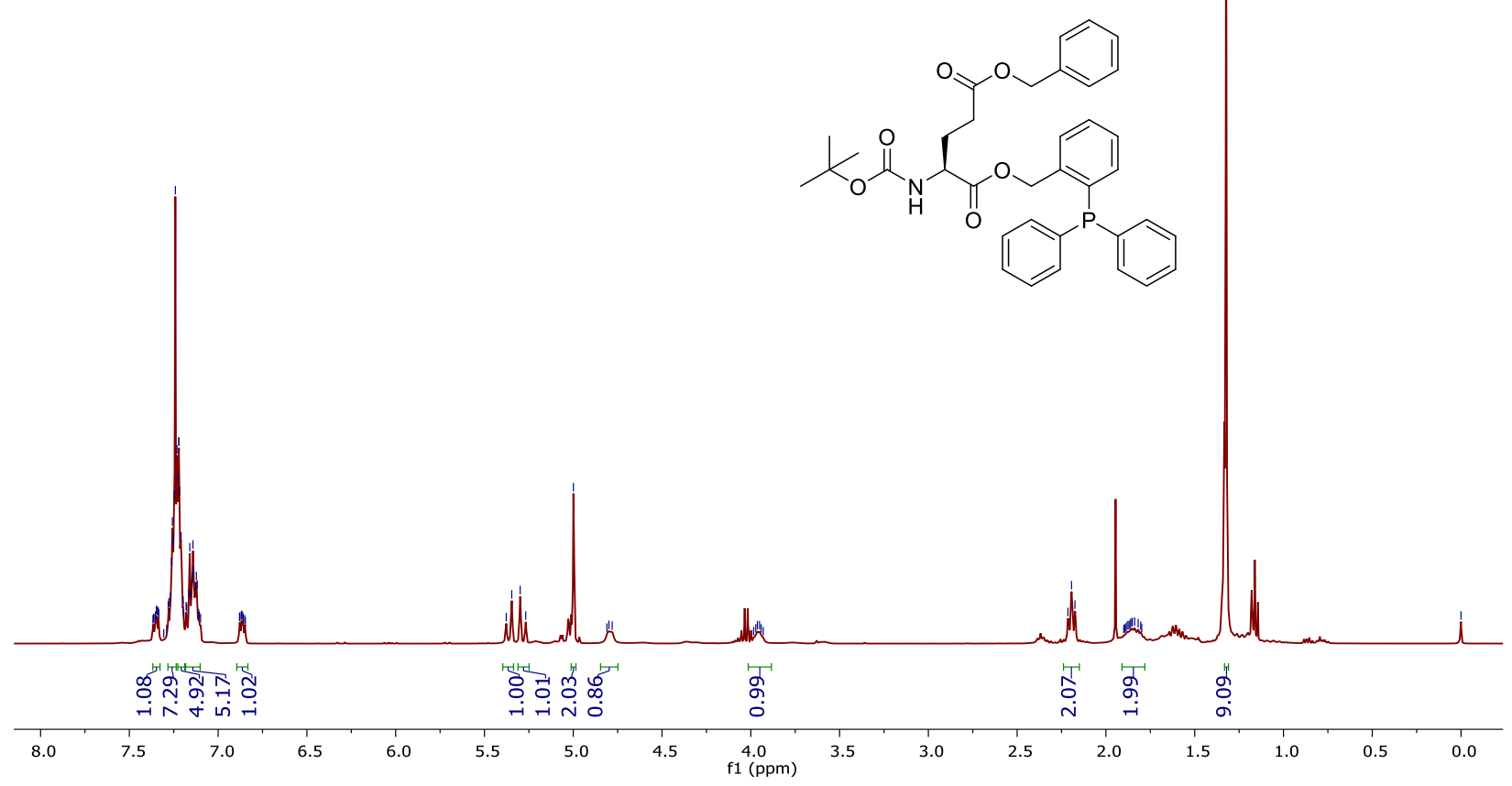

${ }^{13} \mathrm{C}\left\{{ }^{1} \mathrm{H}\right\}$ NMR (101 MHz, Chloroform- $\left.d\right)$ spectra of $\mathbf{4 d}$

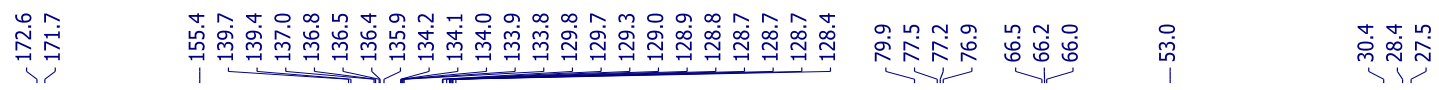
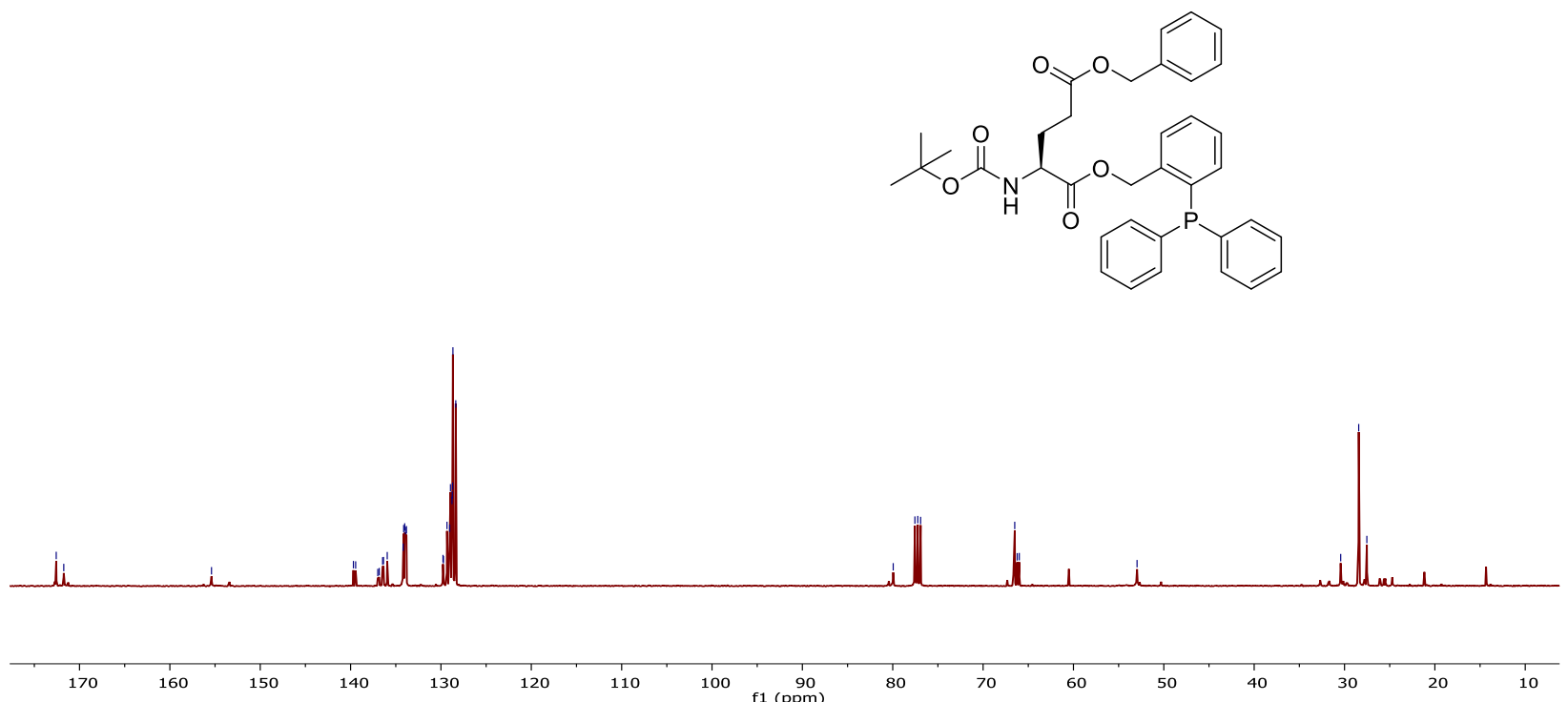
${ }^{31} \mathrm{P}\left\{{ }^{1} \mathrm{H}\right\}$ NMR (162 MHz, Chloroform- $d$ ) spectra of $\mathbf{4 d}$

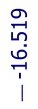

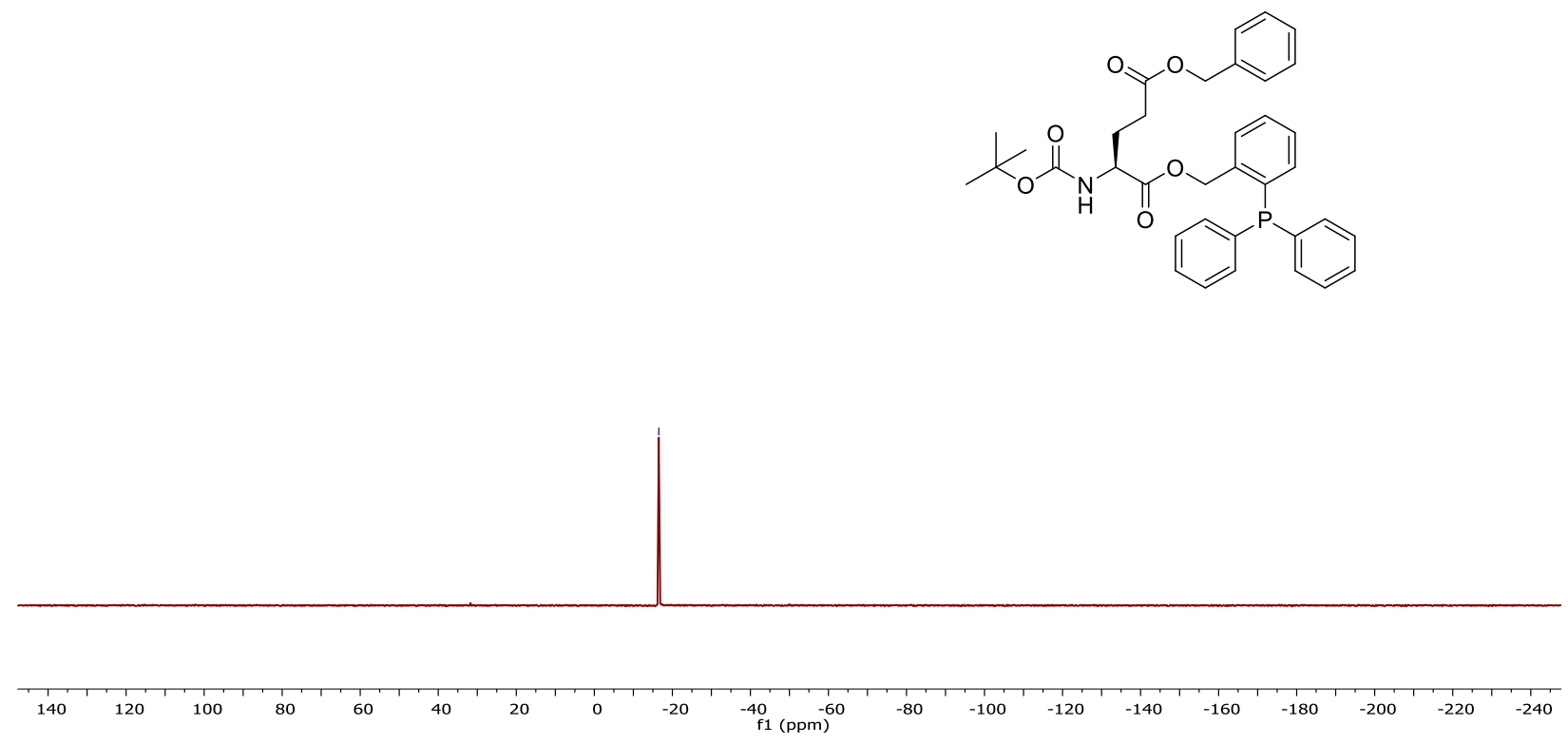

HRMS of $4 d$

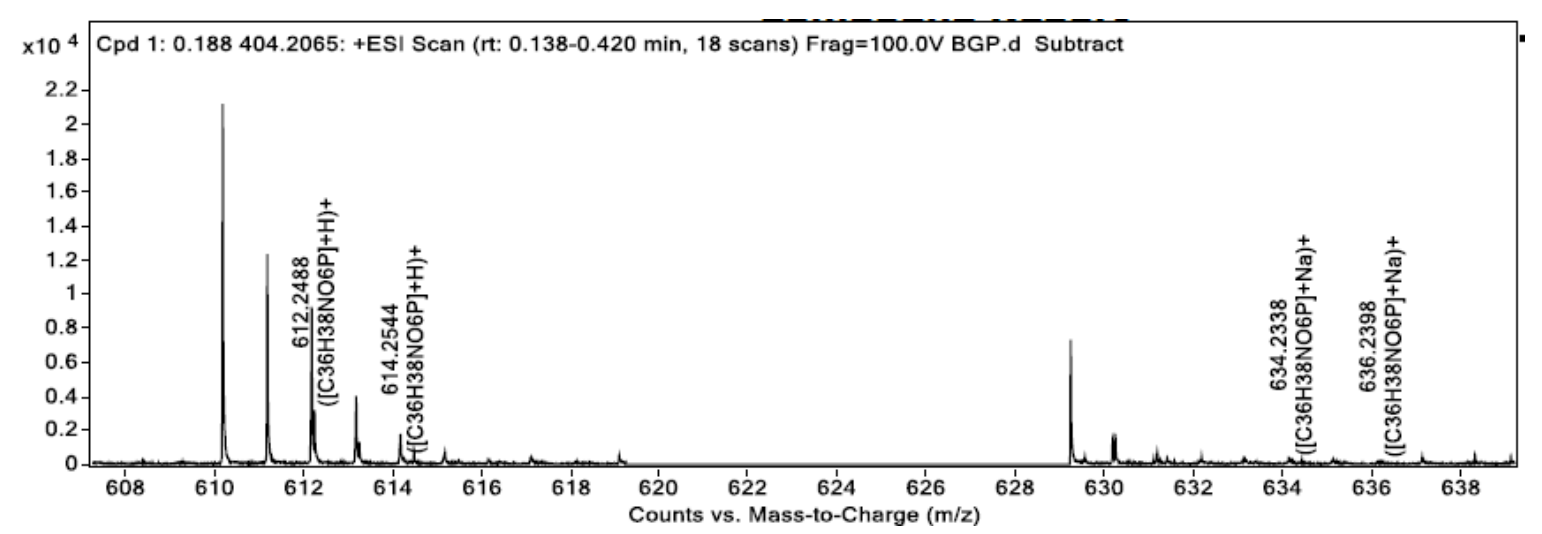


${ }^{1} \mathrm{H}$ NMR (400 MHz, Chloroform- $d$ ) spectra of $\mathbf{4 e}$

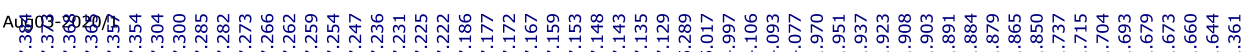<smiles>CC(C)(C)OC(=O)N[C@@H](CCC(=O)O)C(=O)OCc1ccccc1P(c1ccccc1)c1ccccc1</smiles>

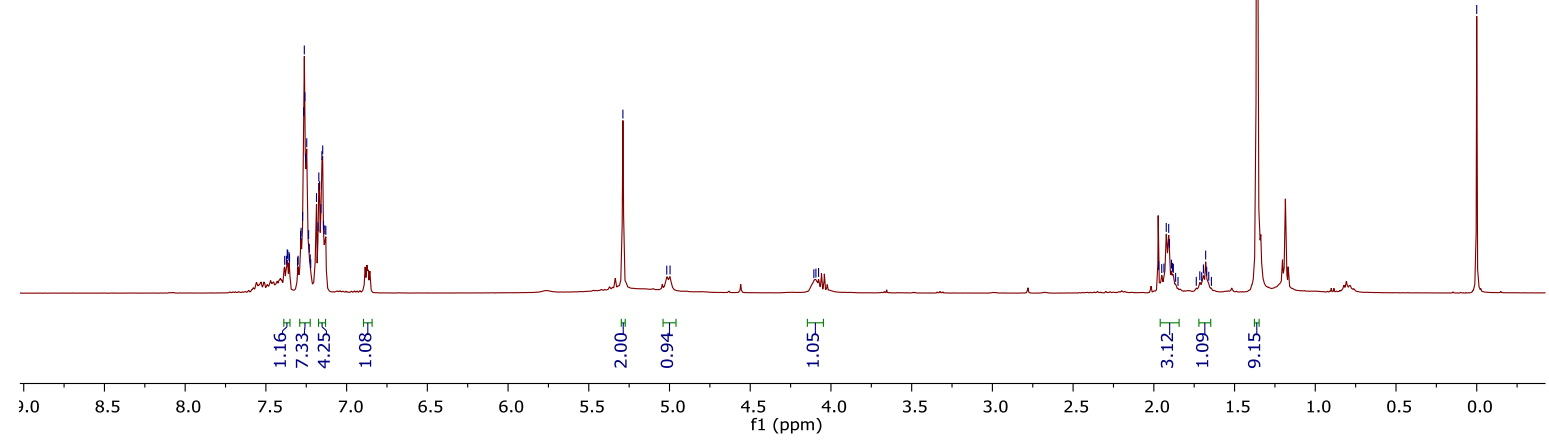

${ }^{13} \mathrm{C}\left\{{ }^{1} \mathrm{H}\right\}$ NMR $(101 \mathrm{MHz}$, Chloroform- $d$ ) spectra of $\mathbf{4 e}$ 等<smiles>CC(C)(C)OC(=O)N[C@@H](CCC(=O)O)C(=O)OCc1ccccc1P(c1ccccc1)c1ccccc1</smiles>
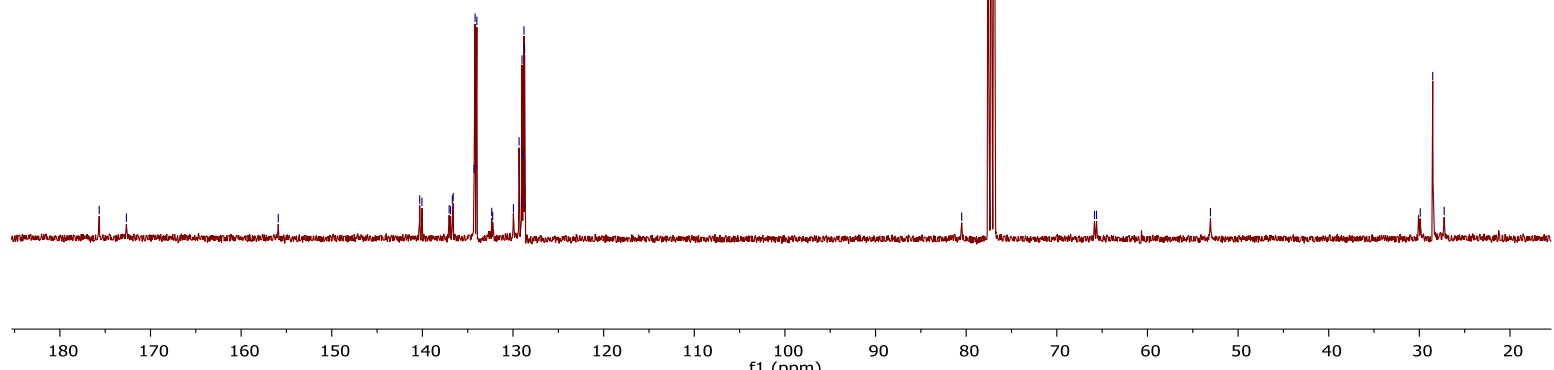
${ }^{31} \mathrm{P}\left\{{ }^{1} \mathrm{H}\right\}$ NMR $\left(162 \mathrm{MHz}\right.$, DMSO- $\left.d_{6}\right) \mathbf{4 e}$<smiles>CC(C)(C)OC(=O)N[C@@H](CCC(=O)O)C(=O)OCc1ccccc1P(c1ccccc1)c1ccccc1</smiles>

\section{$\stackrel{\infty}{\stackrel{0}{0}}$}

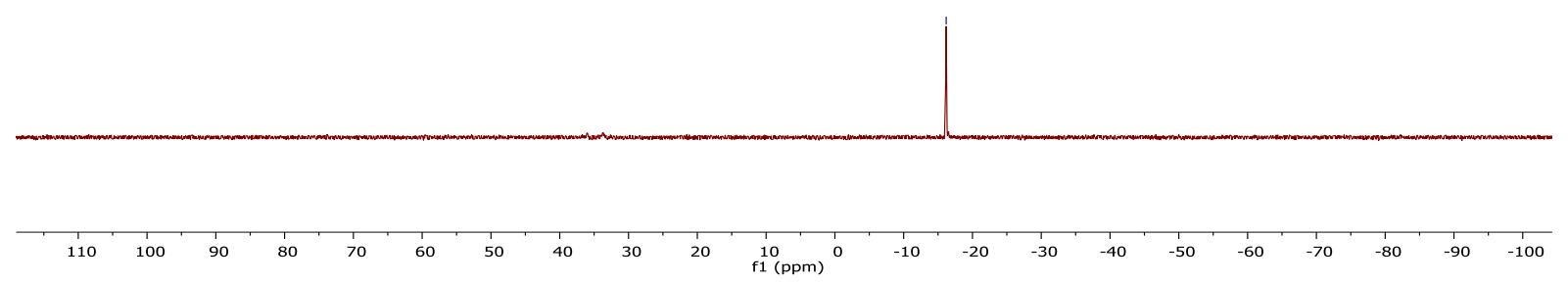

HRMS of 4e

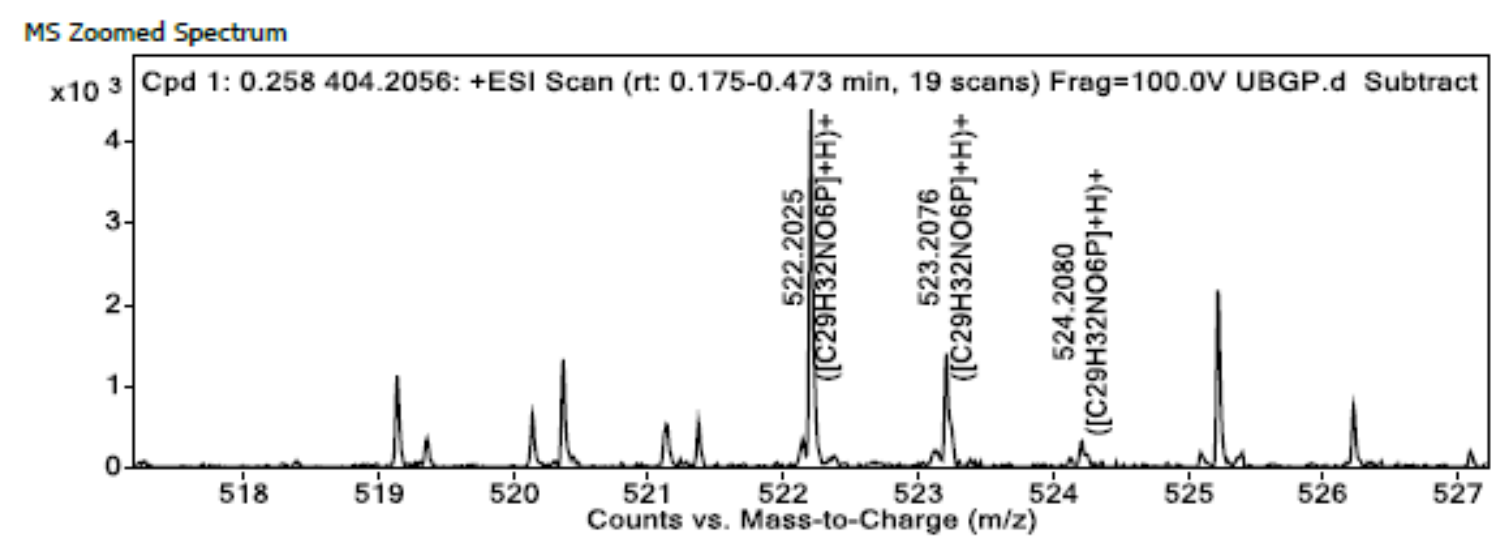




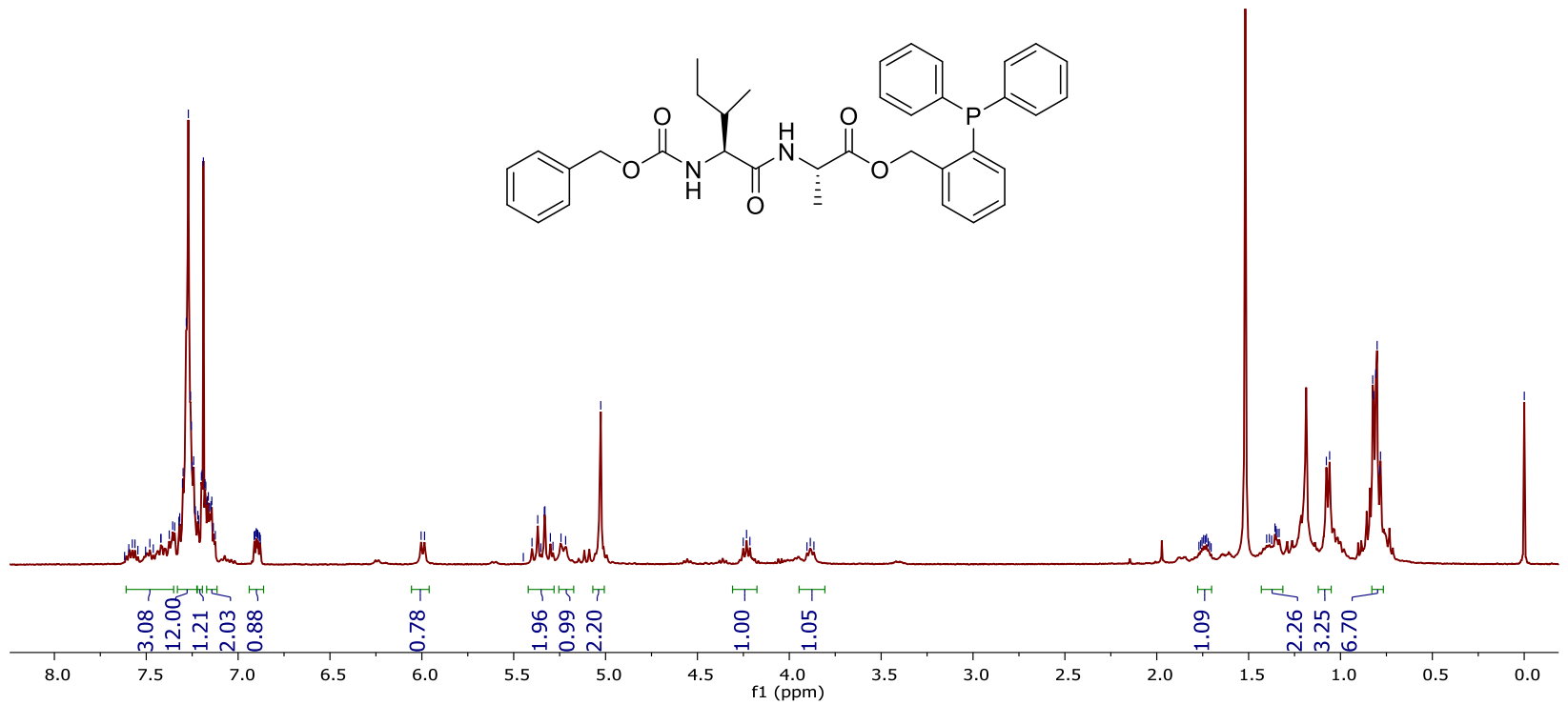

${ }^{13} \mathrm{C}\left\{{ }^{1} \mathrm{H}\right\}$ NMR (101 MHz, Chloroform- $d$ ) spectra of $\mathbf{4 f}$

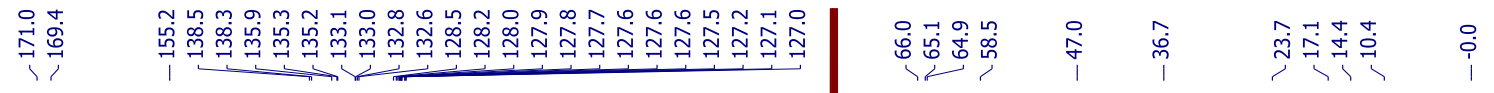
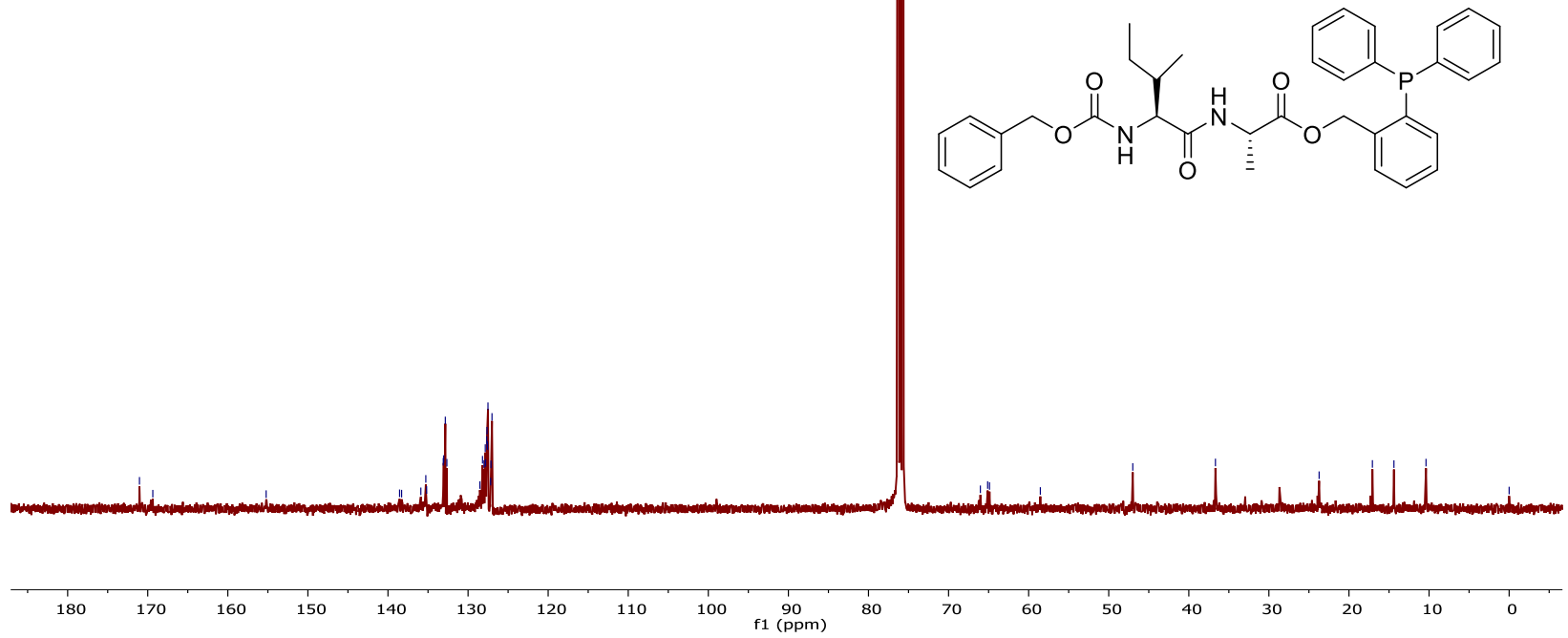
${ }^{31} \mathrm{P}\left\{{ }^{1} \mathrm{H}\right\}$ NMR (162 MHz, Chloroform- $d$ ) spectra of $\mathbf{4 f}$

喜
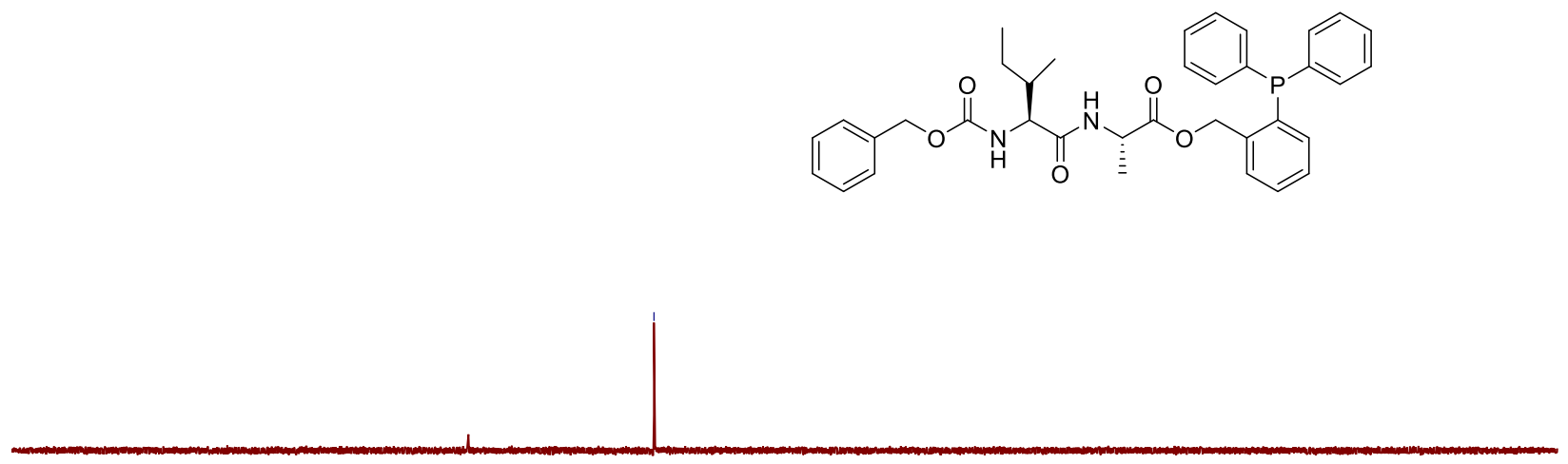

HRMS of $\mathbf{4 f}$

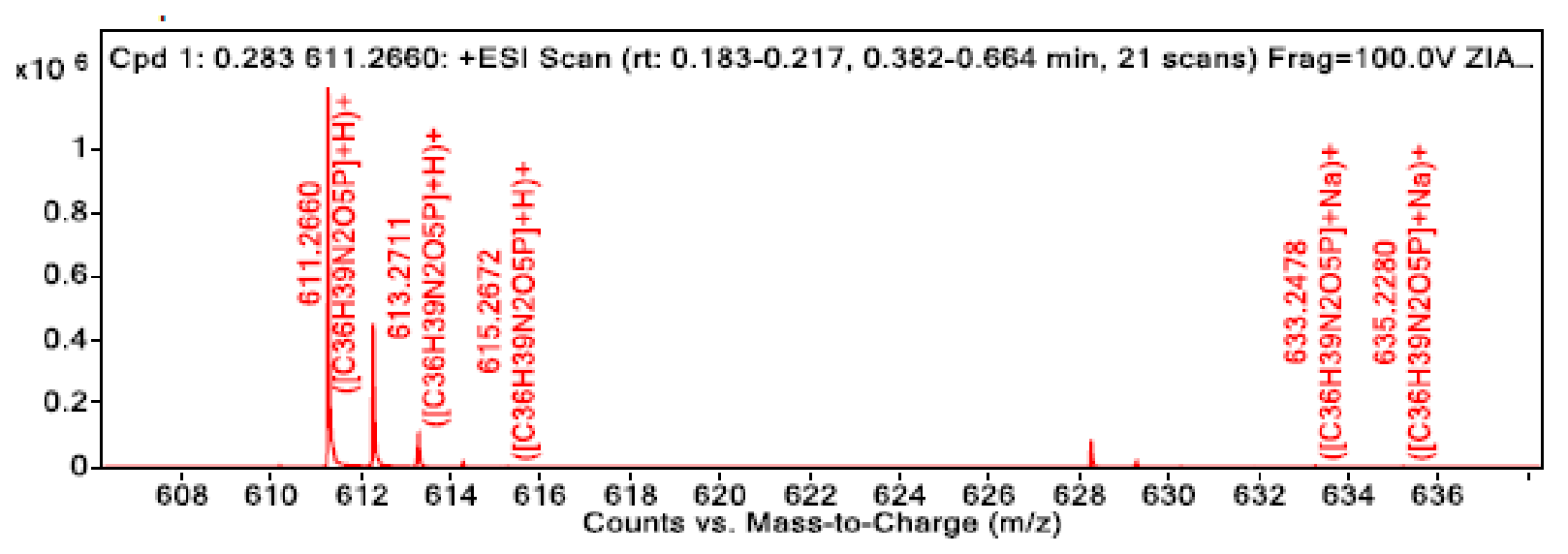


${ }^{1} \mathrm{H}$ NMR (400 MHz, Chloroform- $d$ ) spectra of $\mathbf{4 g}$ (Crude)

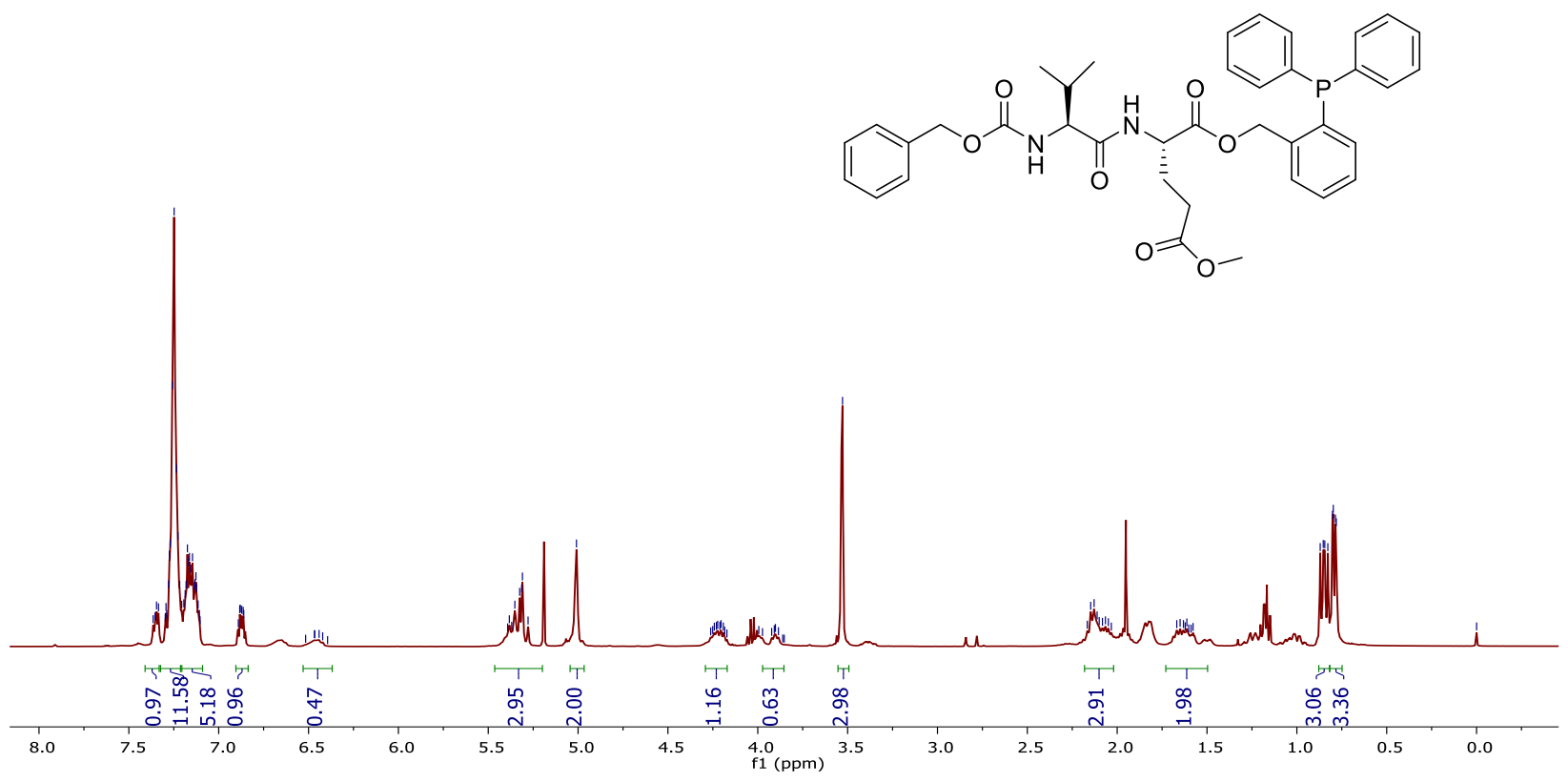

${ }^{13} \mathrm{C}\left\{{ }^{1} \mathrm{H}\right\}$ NMR (101 MHz, Chloroform- $d$ ) spectra of $\mathbf{4 g}$

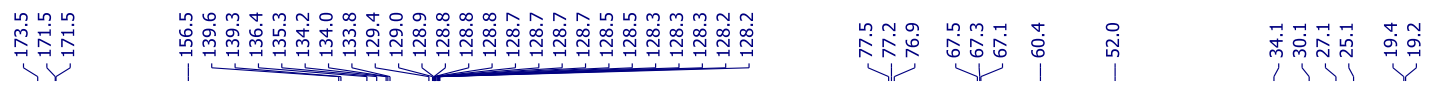
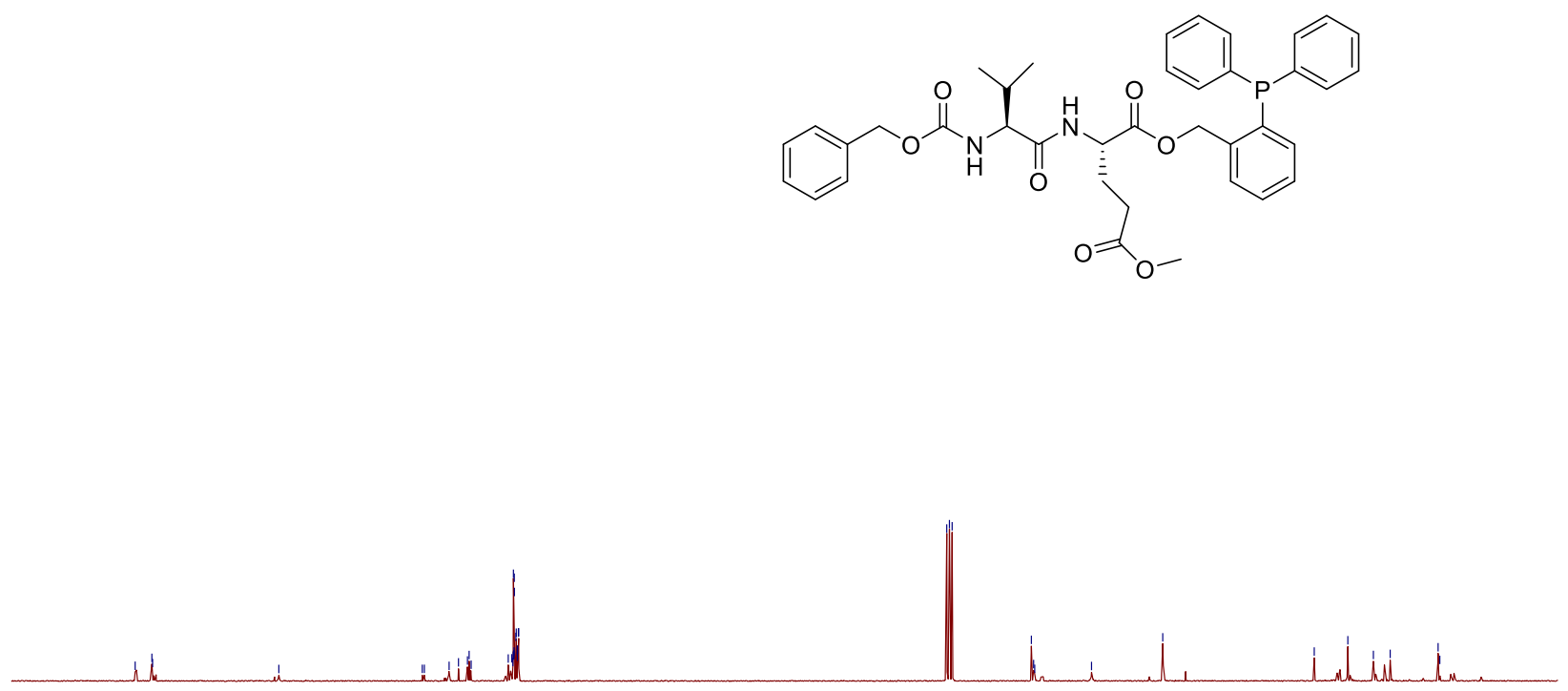

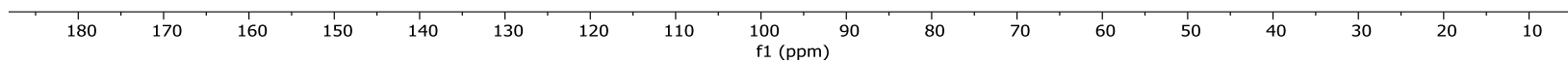


${ }^{31} \mathrm{P}\left\{{ }^{1} \mathrm{H}\right\}$ NMR (162 MHz, Chloroform- $d$ ) spectra of $4 \mathbf{g}$ (Crude)

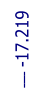<smiles>COC(=O)CC[C@H](NC(=O)C(NC(=O)OCc1ccccc1)C(C)C)C(=O)OCc1ccccc1P(c1ccccc1)c1ccccc1</smiles>

HRMS of $\mathbf{4 g}$

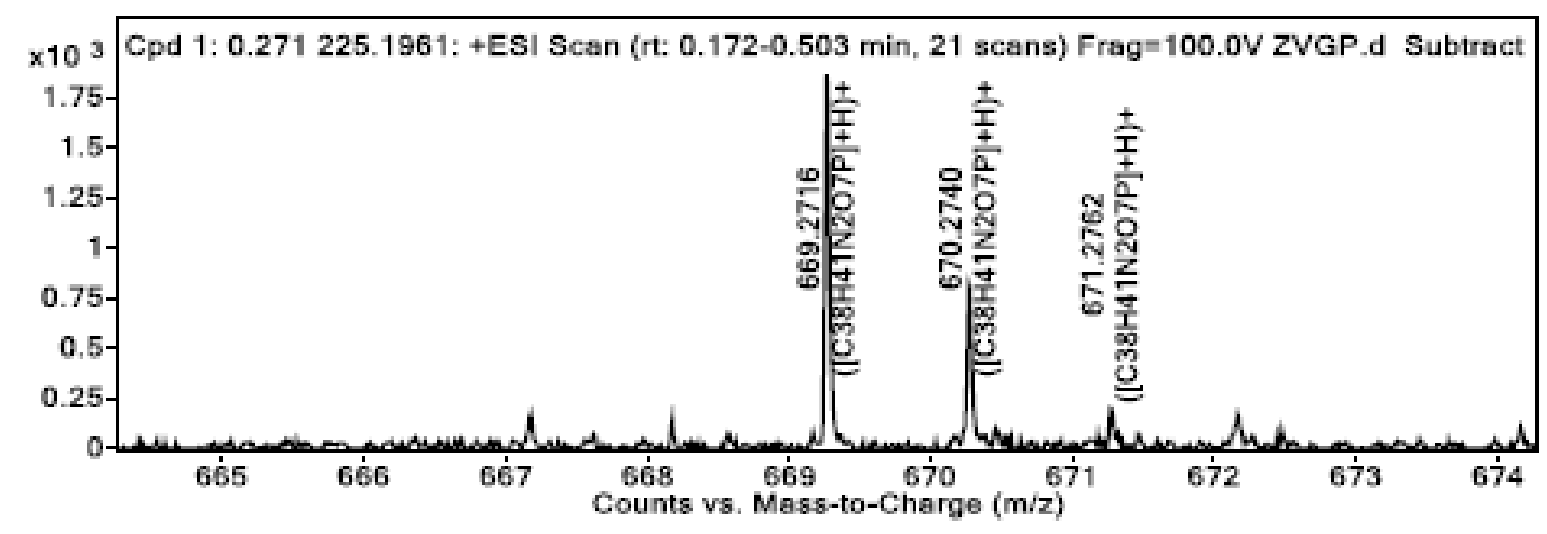


${ }^{1} \mathrm{H}$ NMR (400 MHz, Chloroform- $d$ ) spectra of $\mathbf{4 h}$ (Crude)

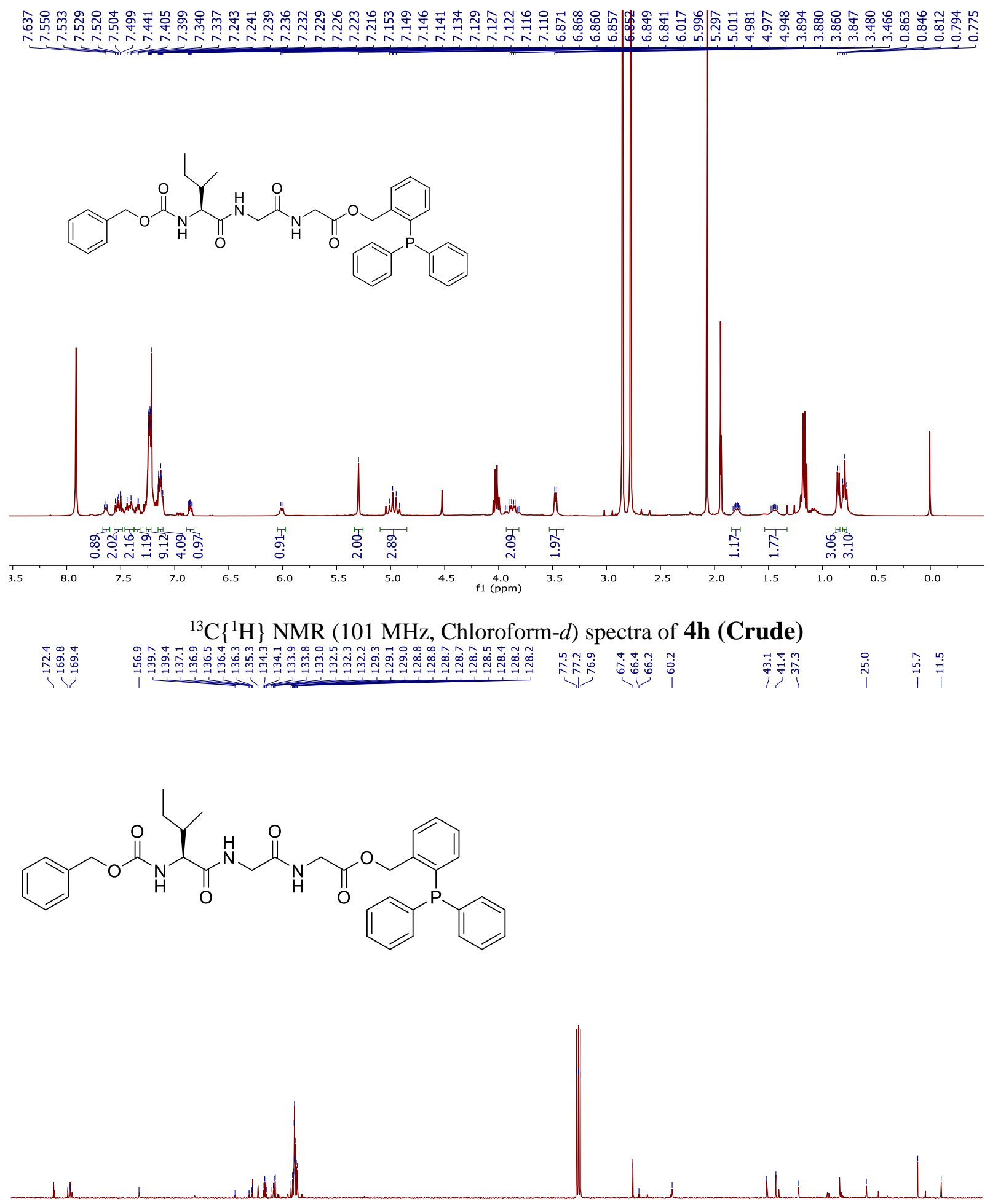

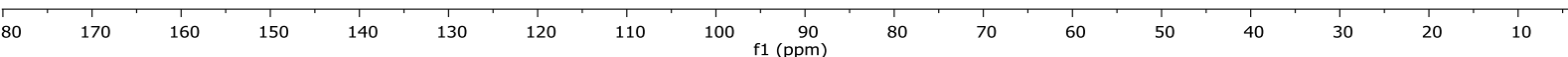




\section{HRMS of $\mathbf{4 h}$}

MS Zoomed Spectrum

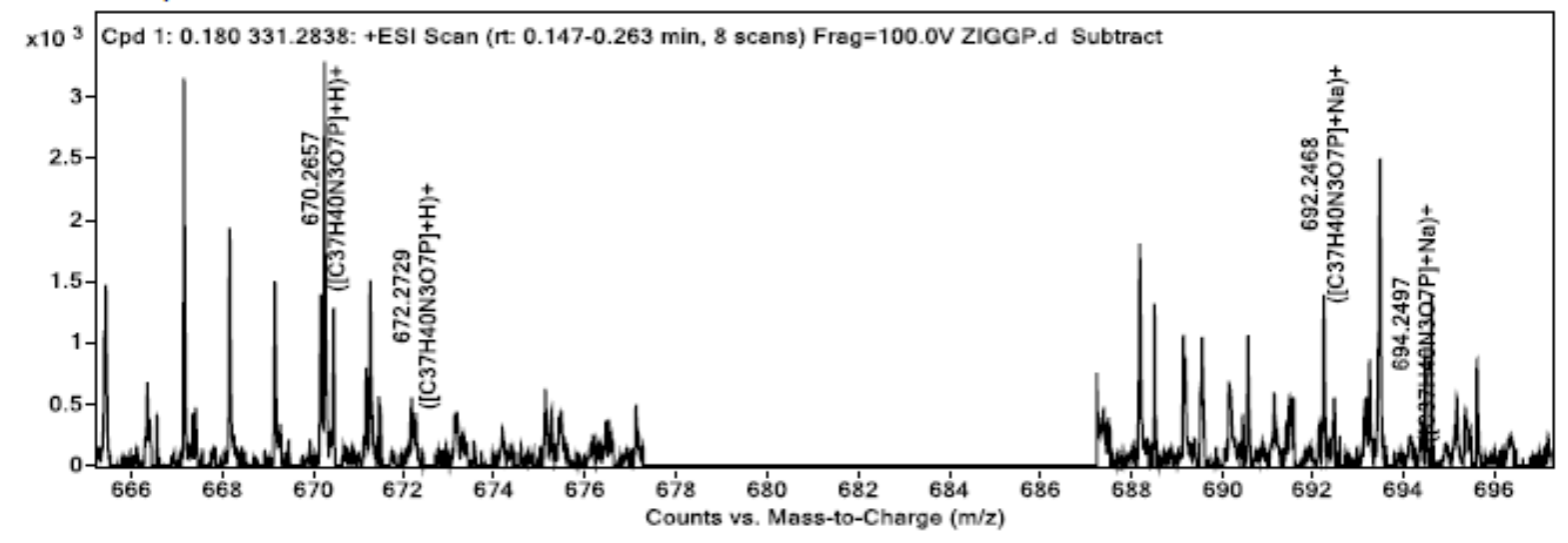

${ }^{1} \mathrm{H}$ NMR (400 MHz, Chloroform- $d$ ) spectra of $\mathbf{4 i}$
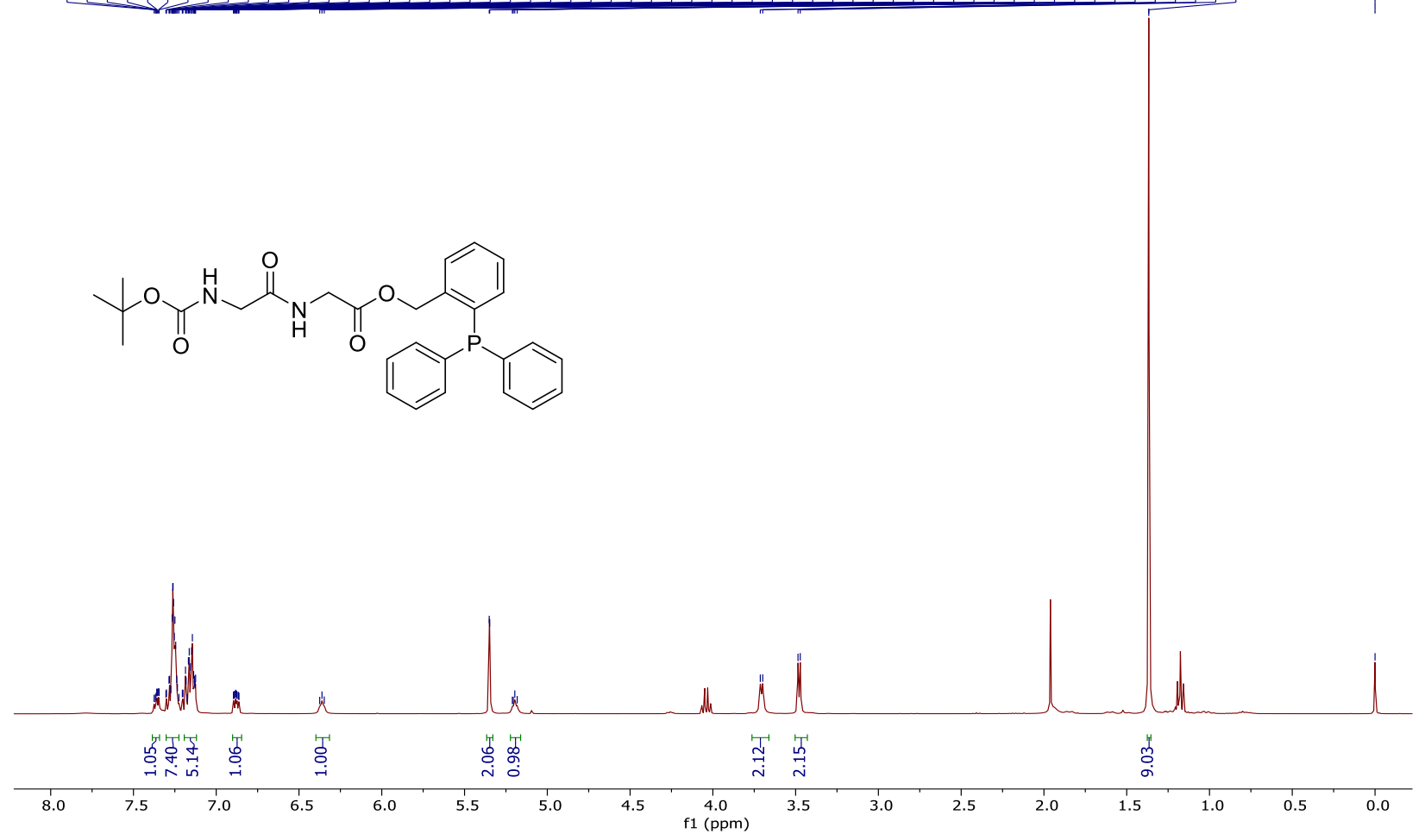
${ }^{13} \mathrm{C}\left\{{ }^{1} \mathrm{H}\right\}$ NMR (101 MHz, Chloroform- $d$ ) spectra of $\mathbf{4 i}$

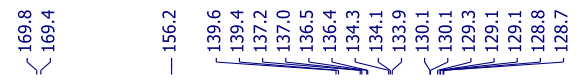

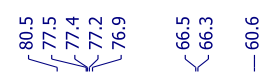

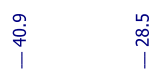<smiles>CC(C)(C)OC(=O)NCC(=O)NCC(=O)OCc1ccccc1P(c1ccccc1)c1ccccc1</smiles>
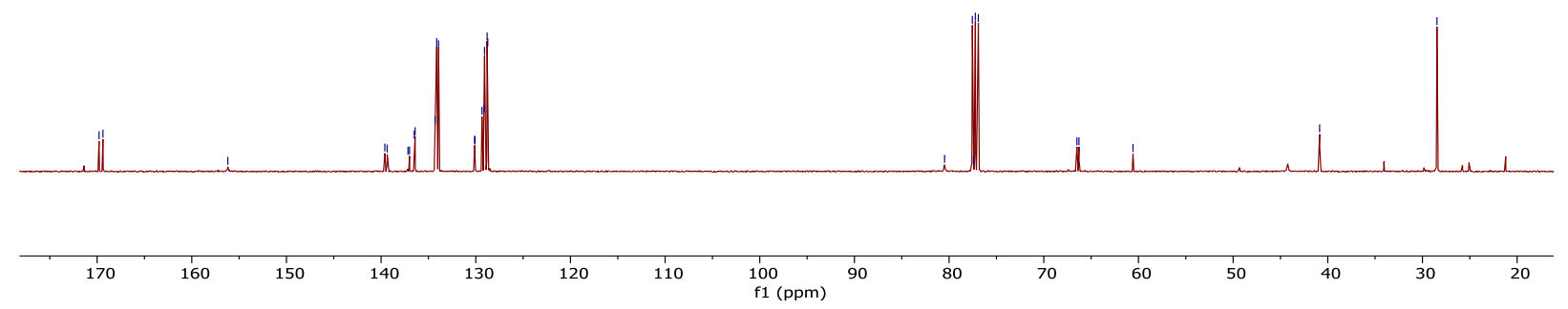

${ }^{31} \mathrm{P}\left\{{ }^{1} \mathrm{H}\right\}$ NMR (162 MHz, Chloroform- $d$ ) spectra of $\mathbf{4 i}$
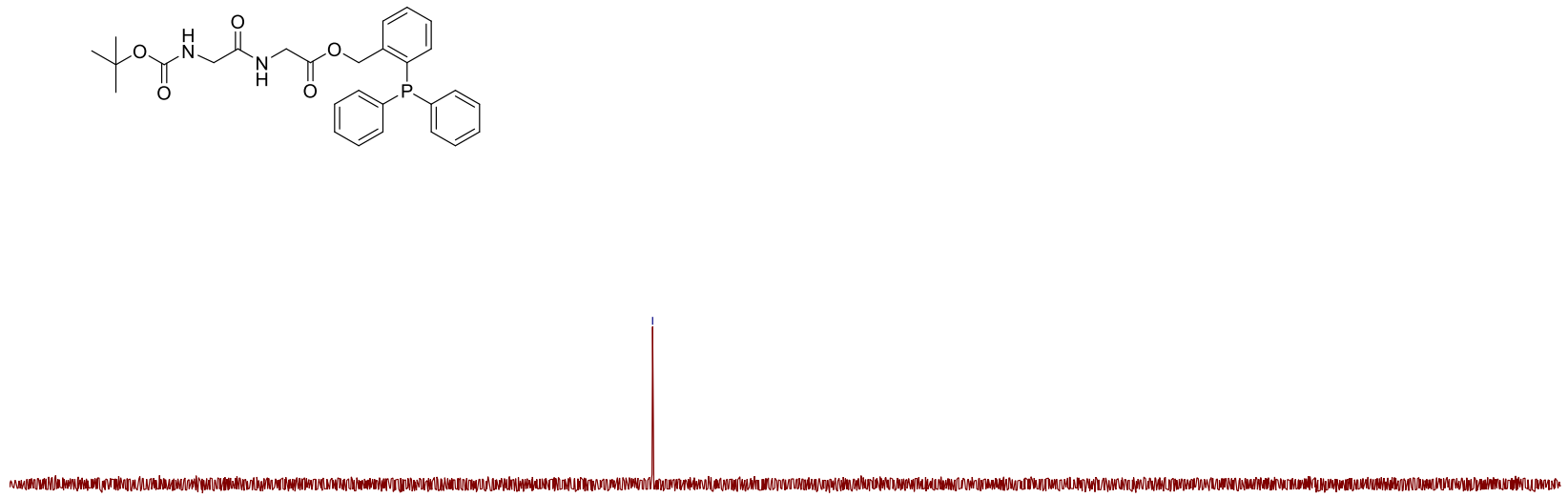

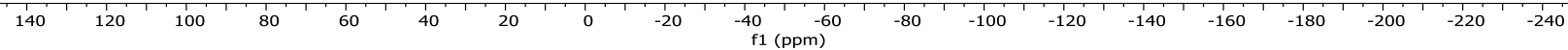




\section{HRMS of $\mathbf{4 i}$}

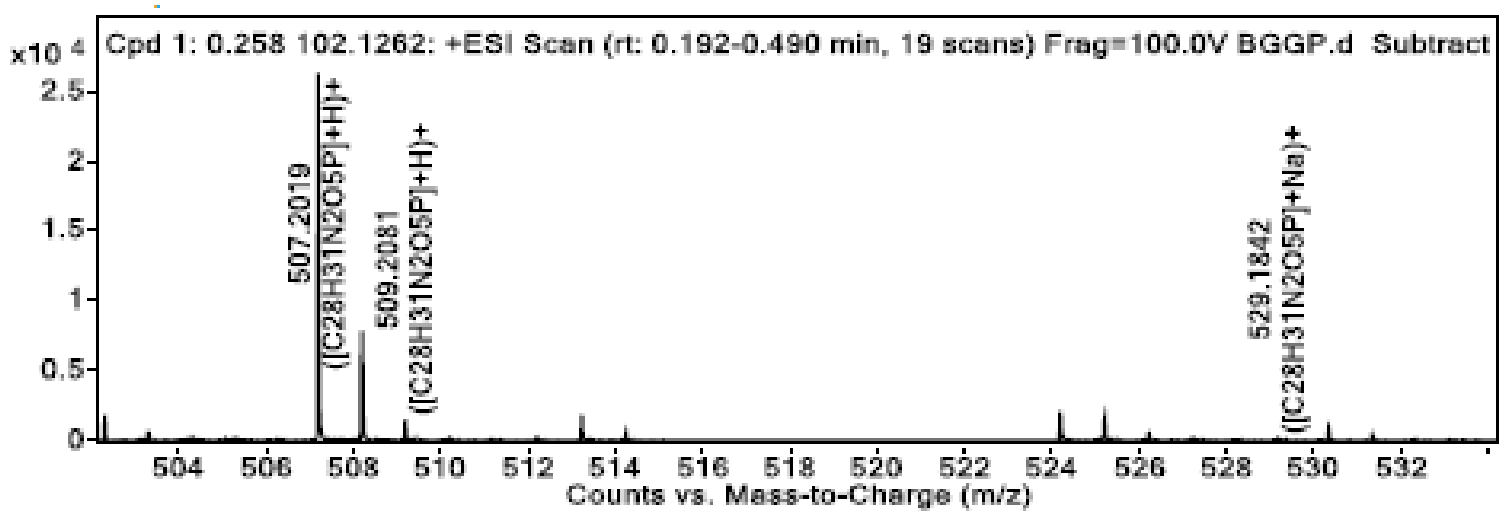

${ }^{1} \mathrm{H}$ NMR (400 MHz, DMSO- $d_{6}$ ) spectra of 4i' (Crude)

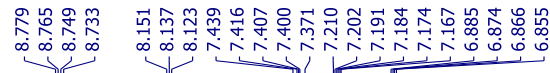

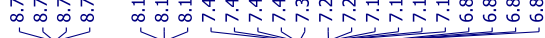

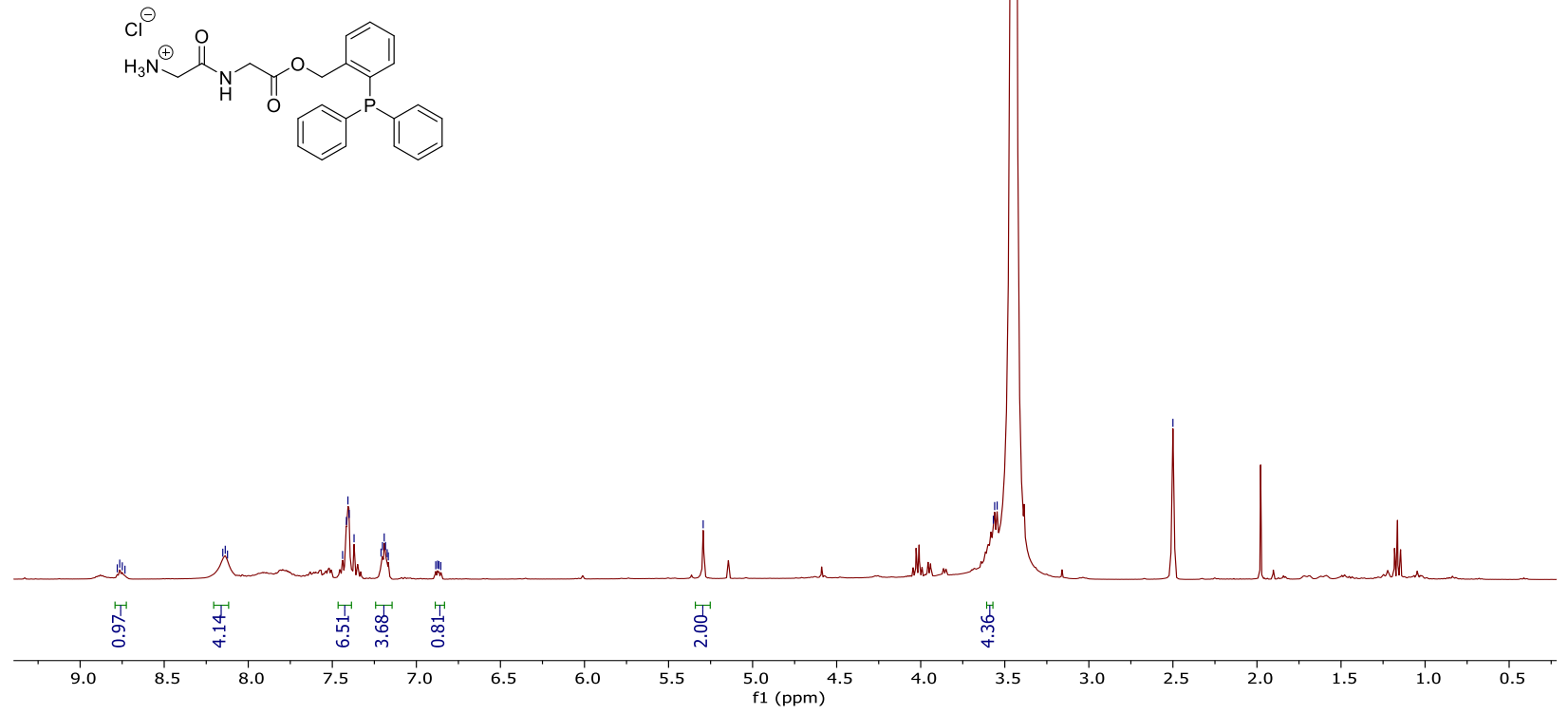

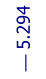

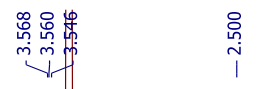


${ }^{31} \mathrm{P}\left\{{ }^{1} \mathrm{H}\right\}$ NMR (162 MHz, DMSO- $\left.d_{6}\right)$ 4i' (Crude)

离
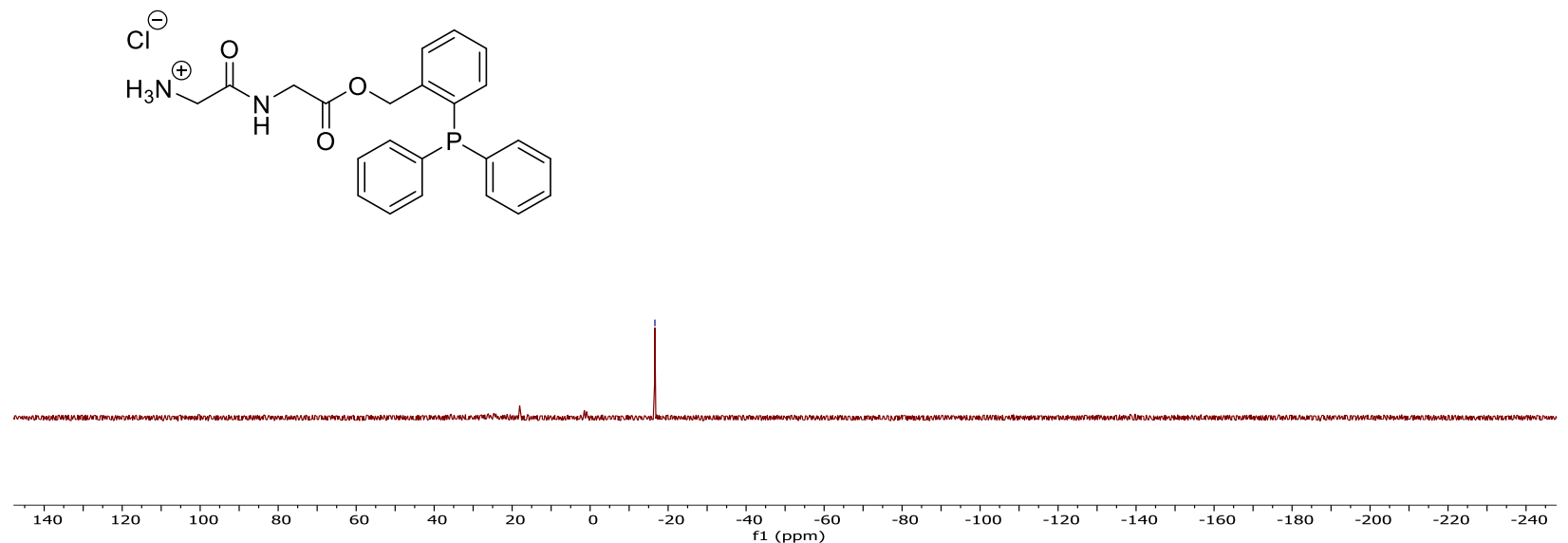

\section{Original NMR spectra of $\mathbf{5 a - 5 i}$}

${ }^{1} \mathrm{H}$ NMR (400 MHz, Chloroform- $d$ ) spectra of 5a

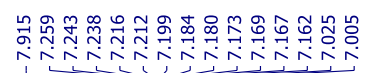

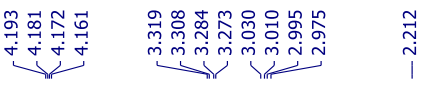
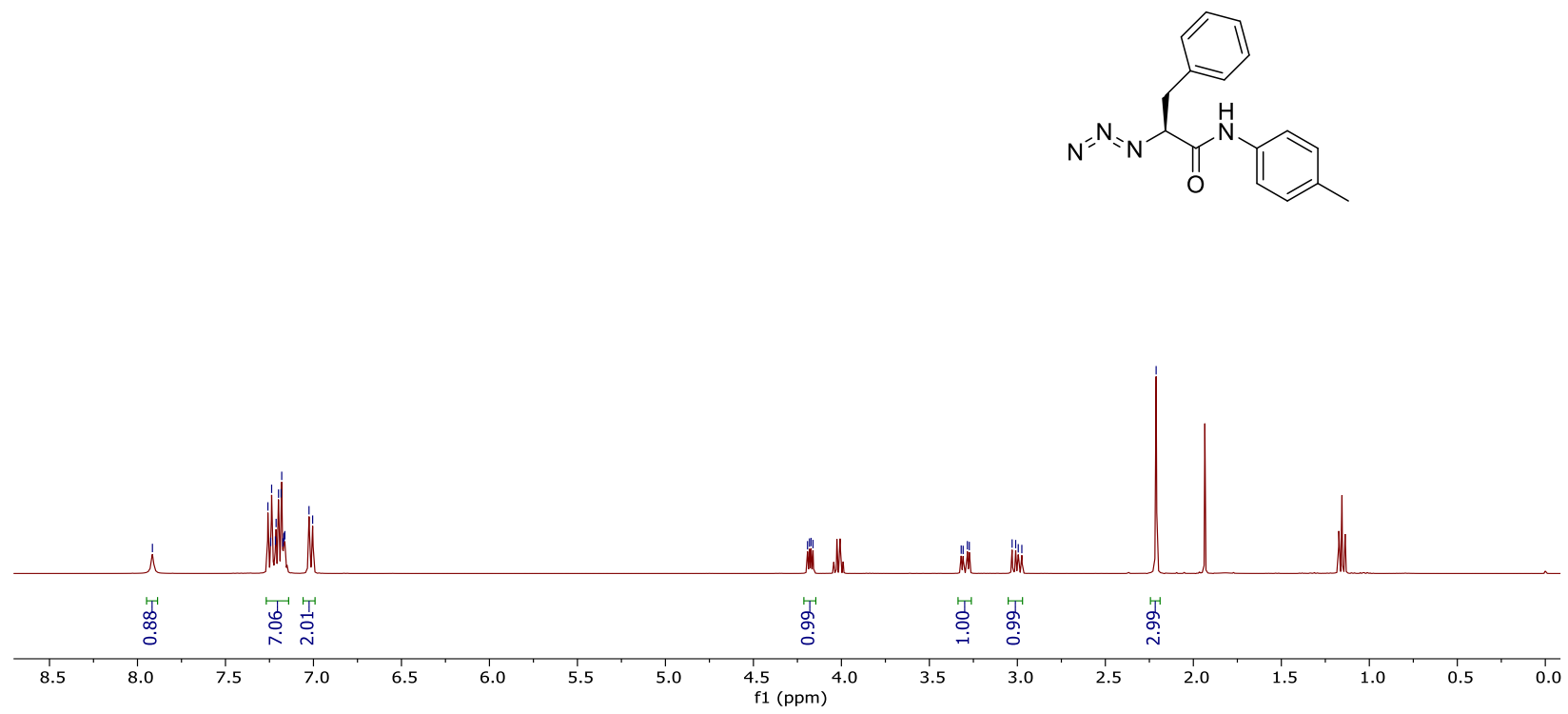
${ }^{13} \mathrm{C}\left\{{ }^{1} \mathrm{H}\right\}$ NMR (101 MHz, Chloroform- $d$ ) spectra of $\mathbf{5 a}$

每

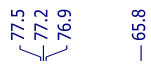

$\stackrel{\substack{\infty \\ i}}{i}$

$N^{=}=N^{2}$
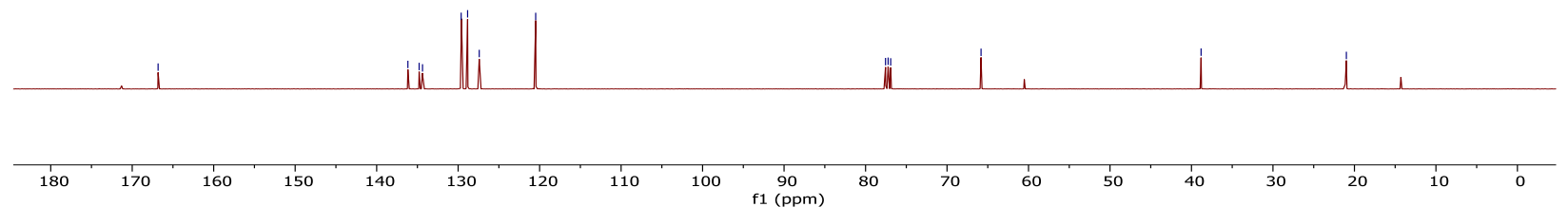

HRMS of 5a

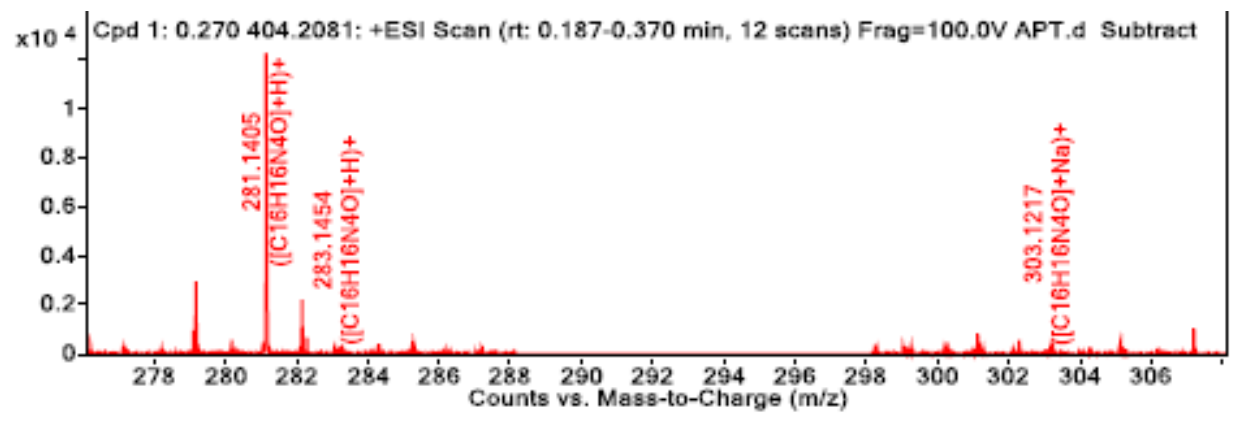


${ }^{1} \mathrm{H}$ NMR (400 MHz, Chloroform- $d$ ) spectra of $\mathbf{5 b}$
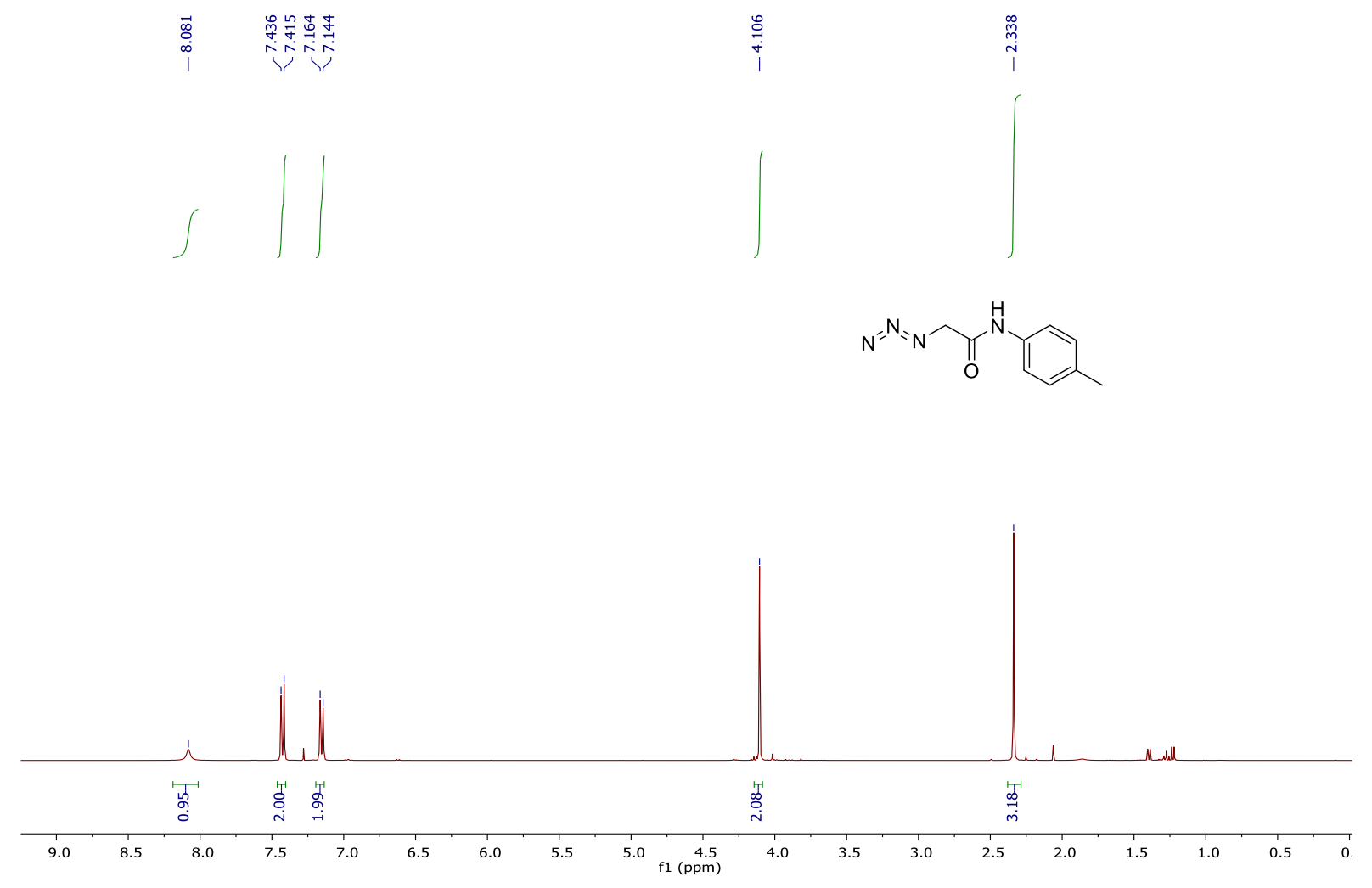

\section{${ }^{13} \mathrm{C}\left\{{ }^{1} \mathrm{H}\right\}$ NMR (101 MHz, Chloroform- $d$ ) spectra of $\mathbf{5 b}$}
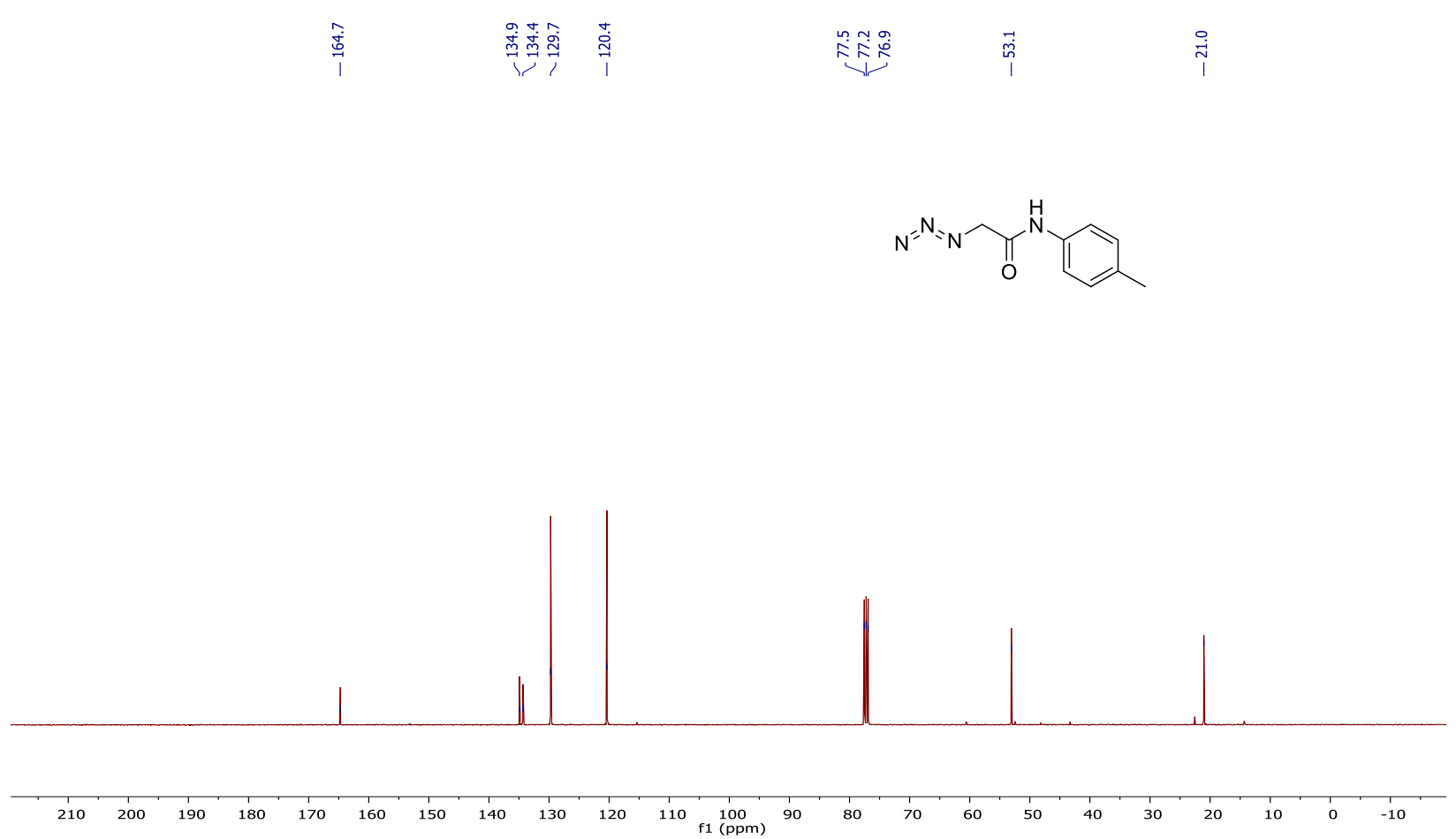
${ }^{1} \mathrm{H}$ NMR (400 MHz, Chloroform- $d$ ) spectra of 5c

家

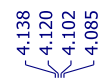

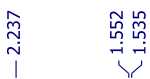
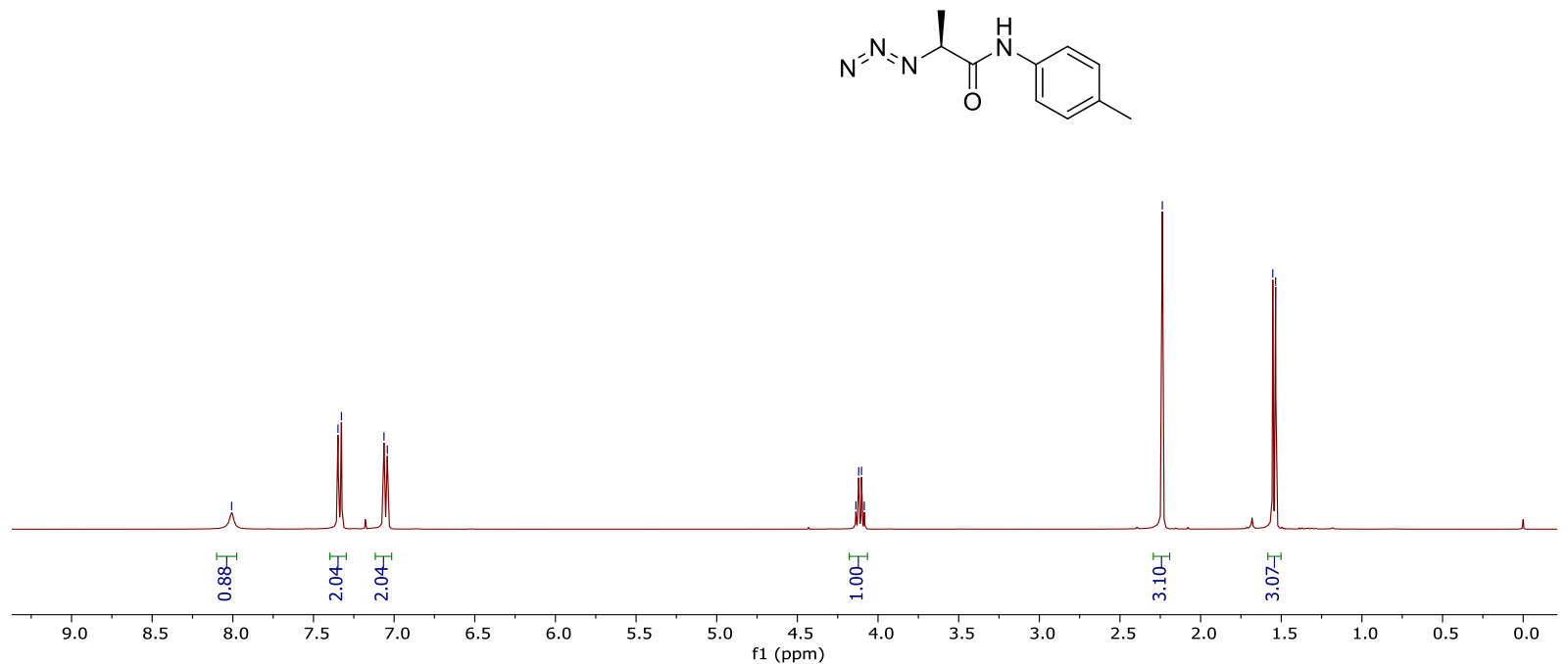

${ }^{13} \mathrm{C}\left\{{ }^{1} \mathrm{H}\right\}$ NMR (101 MHz, Chloroform- $d$ ) spectra of 5c
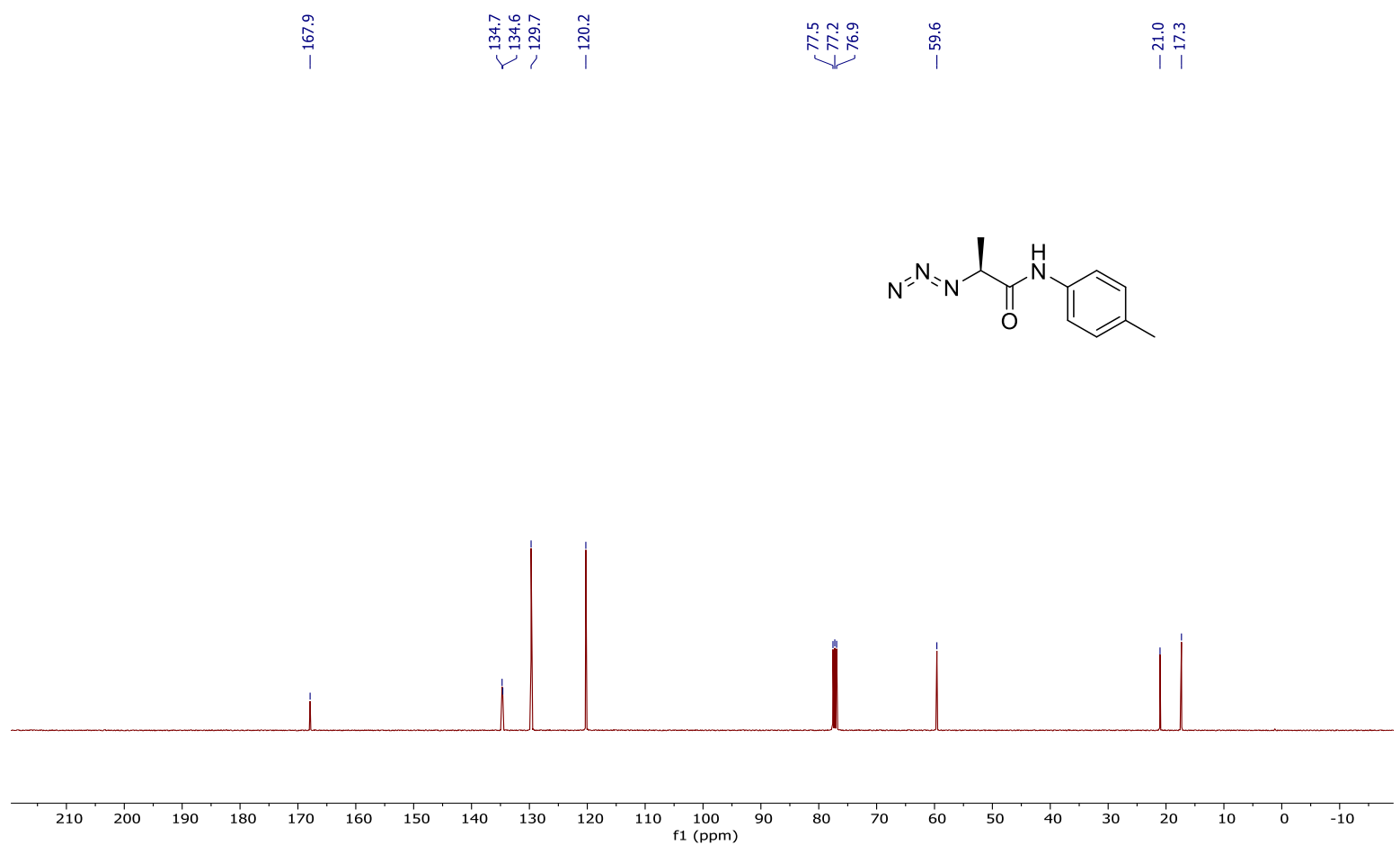


\section{HRMS of 5c}

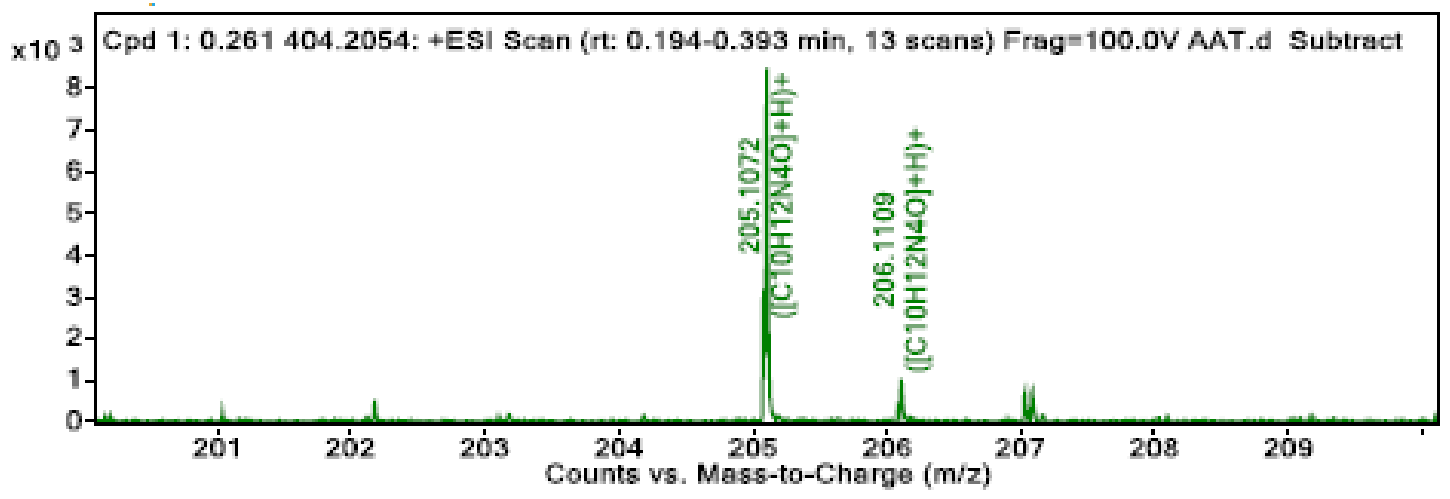

${ }^{1}$ H NMR (400 MHz, Chloroform- $d$ ) spectra of 5d

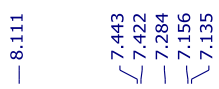

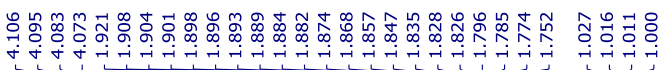
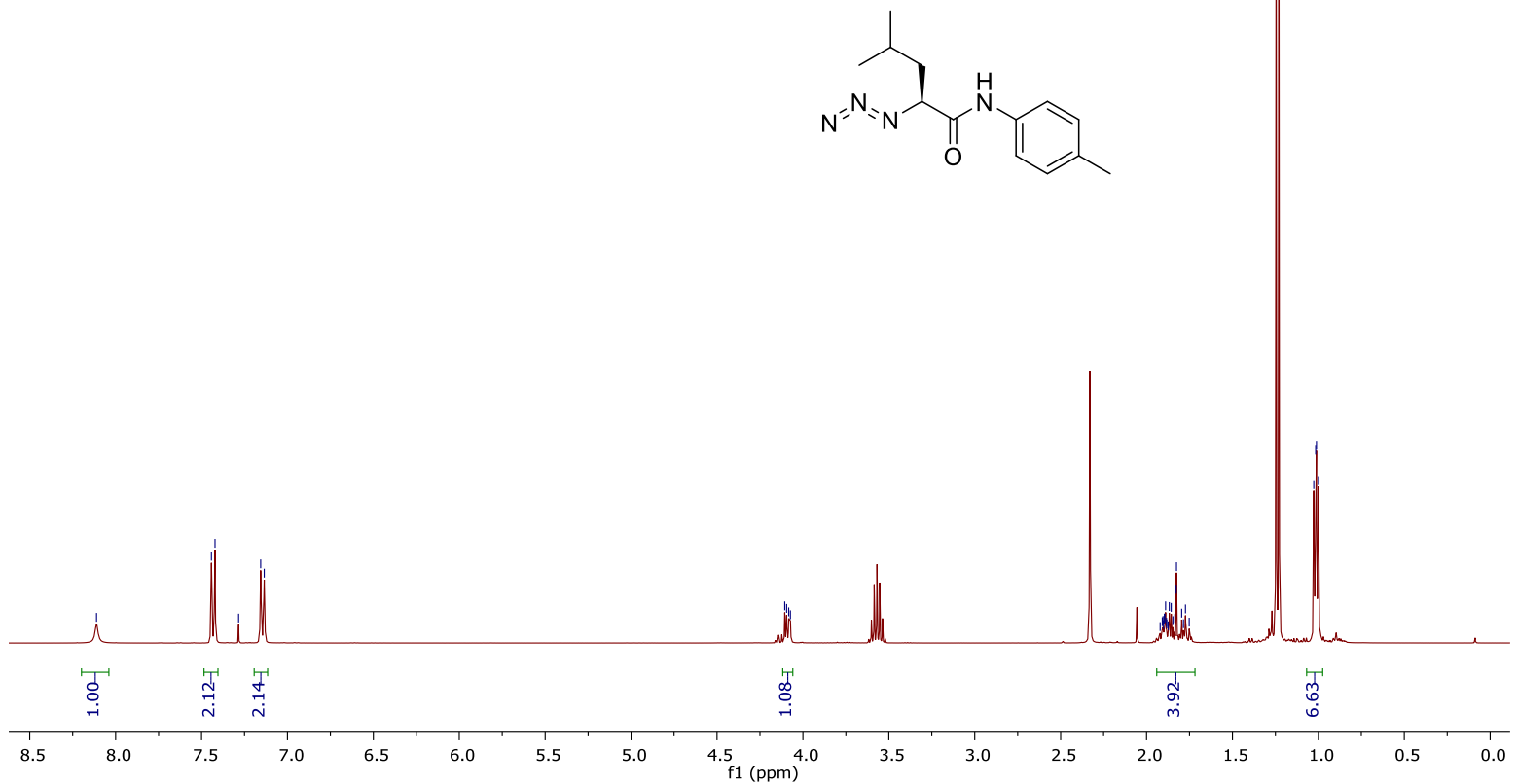
${ }^{13} \mathrm{C}\left\{{ }^{1} \mathrm{H}\right\}$ NMR (101 MHz, Chloroform- $d$ ) spectra of $\mathbf{5 d}$

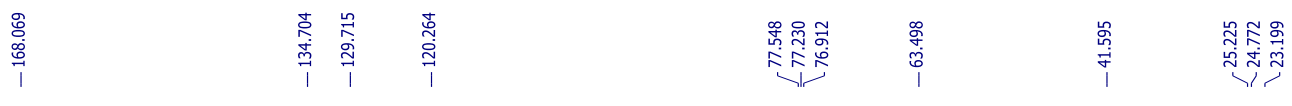
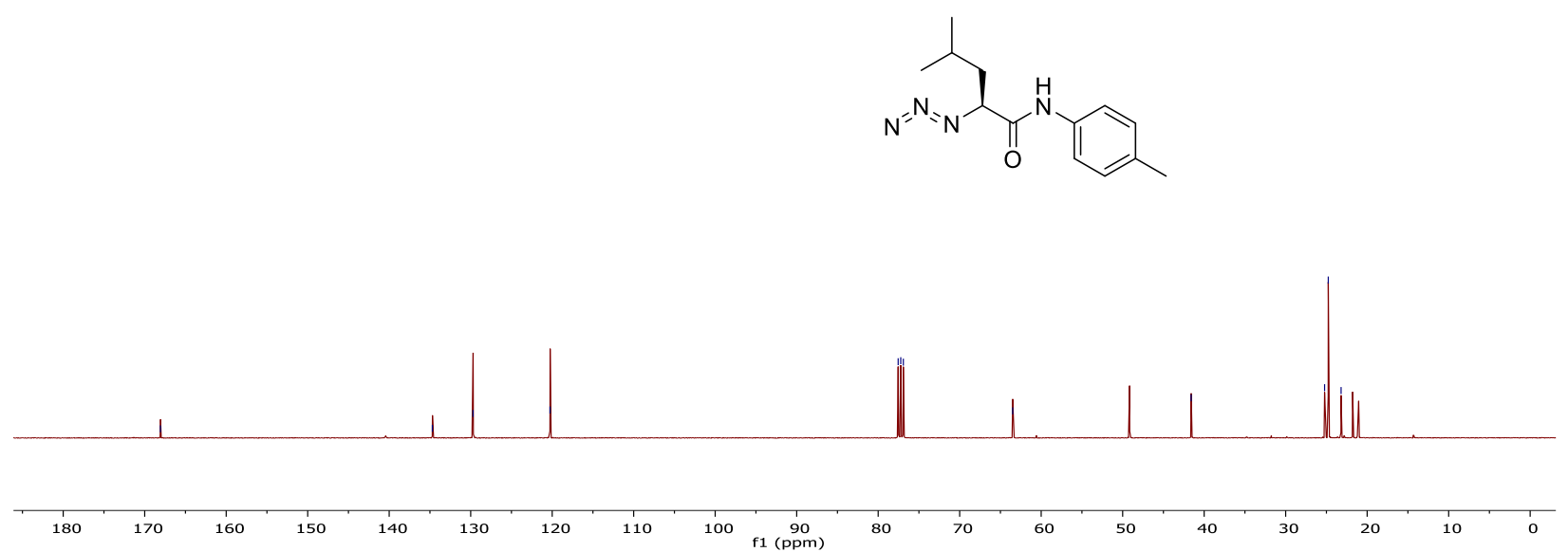

\section{HRMS of 5d}

\section{MS Zoomed Spectrum}

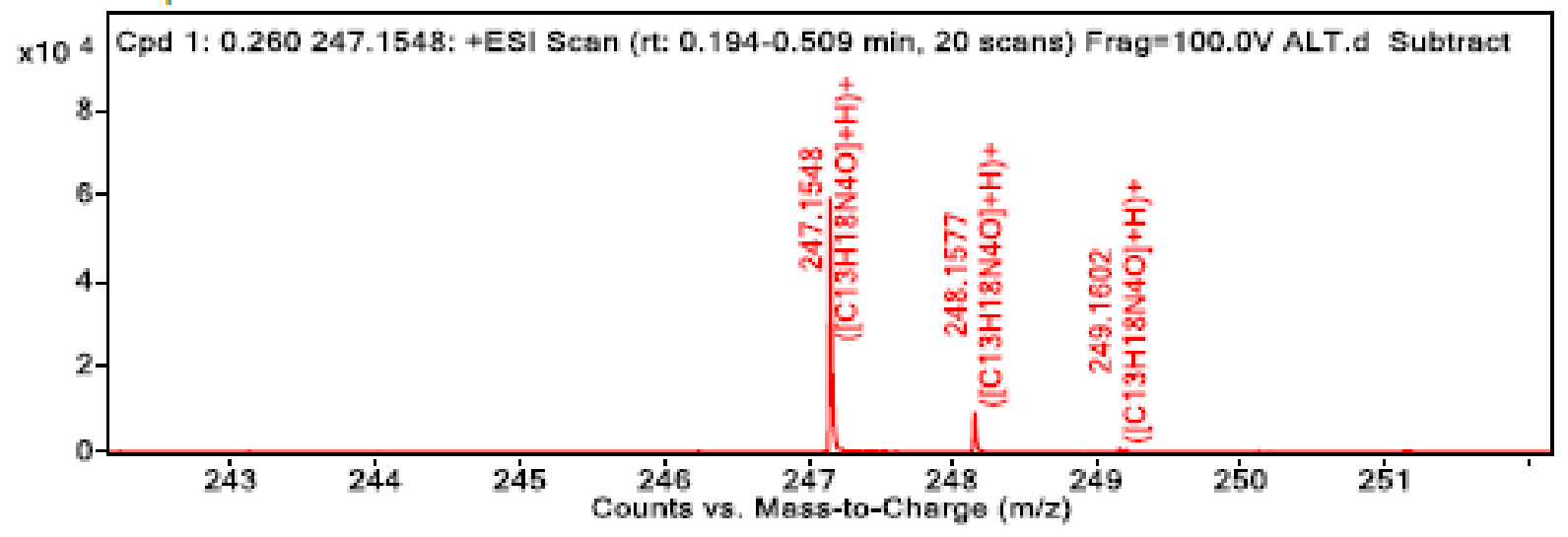


${ }^{1} \mathrm{H}$ NMR (400 MHz, Chloroform- $d$ ) spectra of $\mathbf{5 e}$
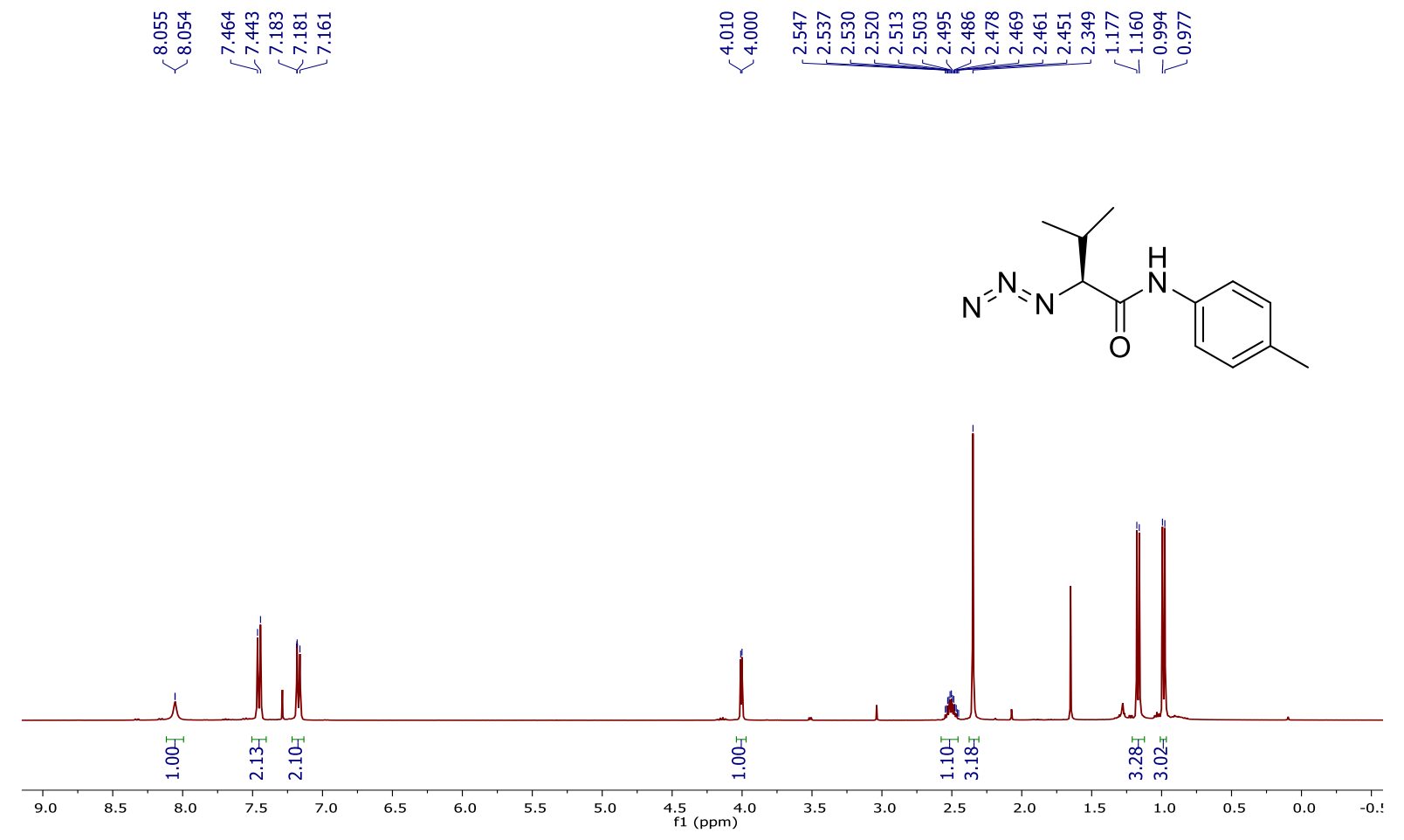

${ }^{13} \mathrm{C}\left\{{ }^{1} \mathrm{H}\right\}$ NMR (101 MHz, Chloroform- $d$ ) spectra of $\mathbf{5 e}$

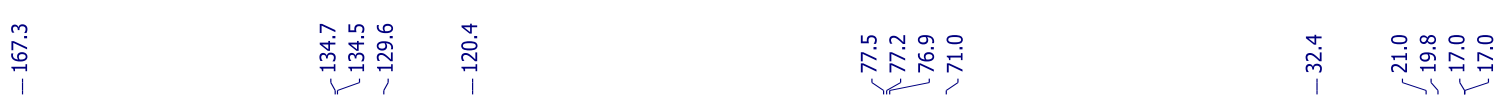
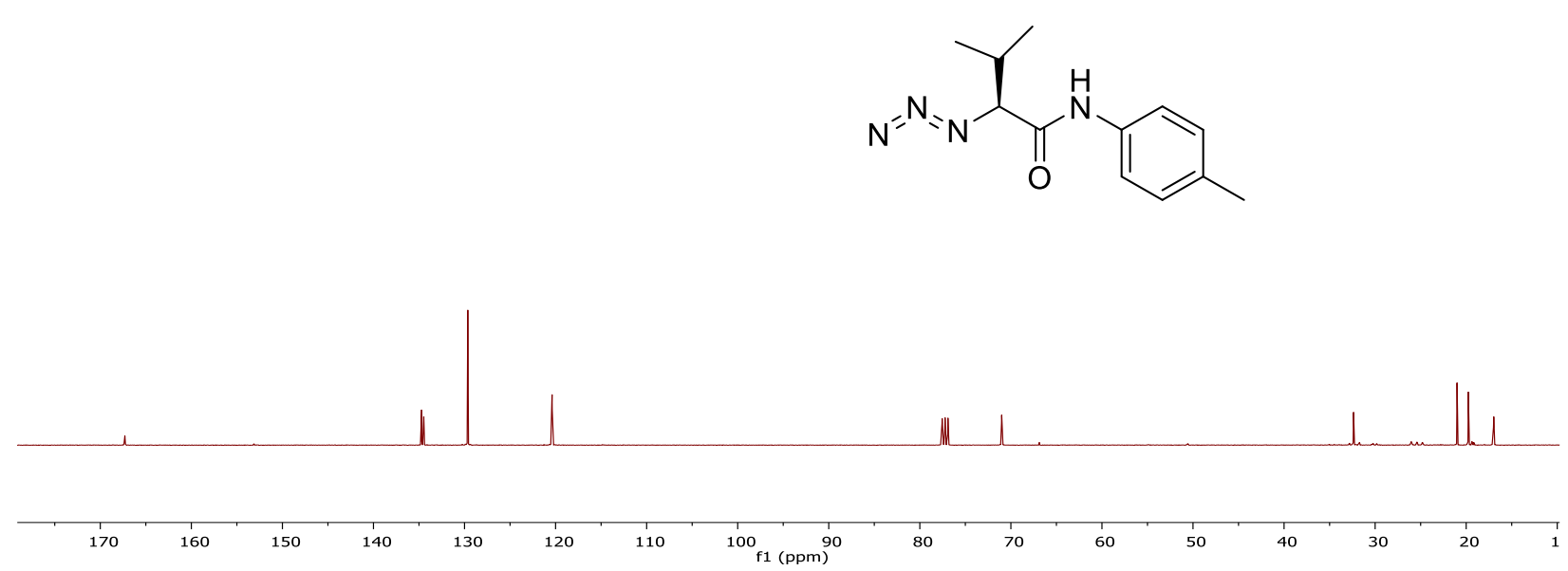


\section{HRMS of $\mathbf{5 e}$}

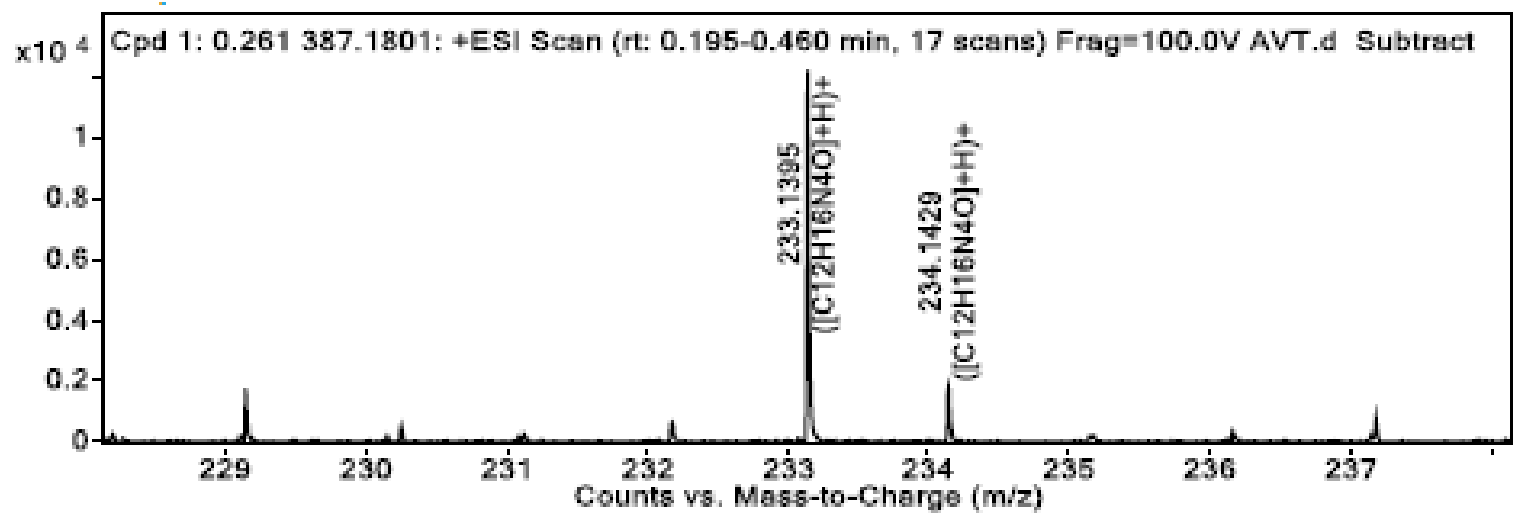

${ }^{1} \mathrm{H}$ NMR (400 MHz, Chloroform- $d$ ) spectra of $\mathbf{5 f}$

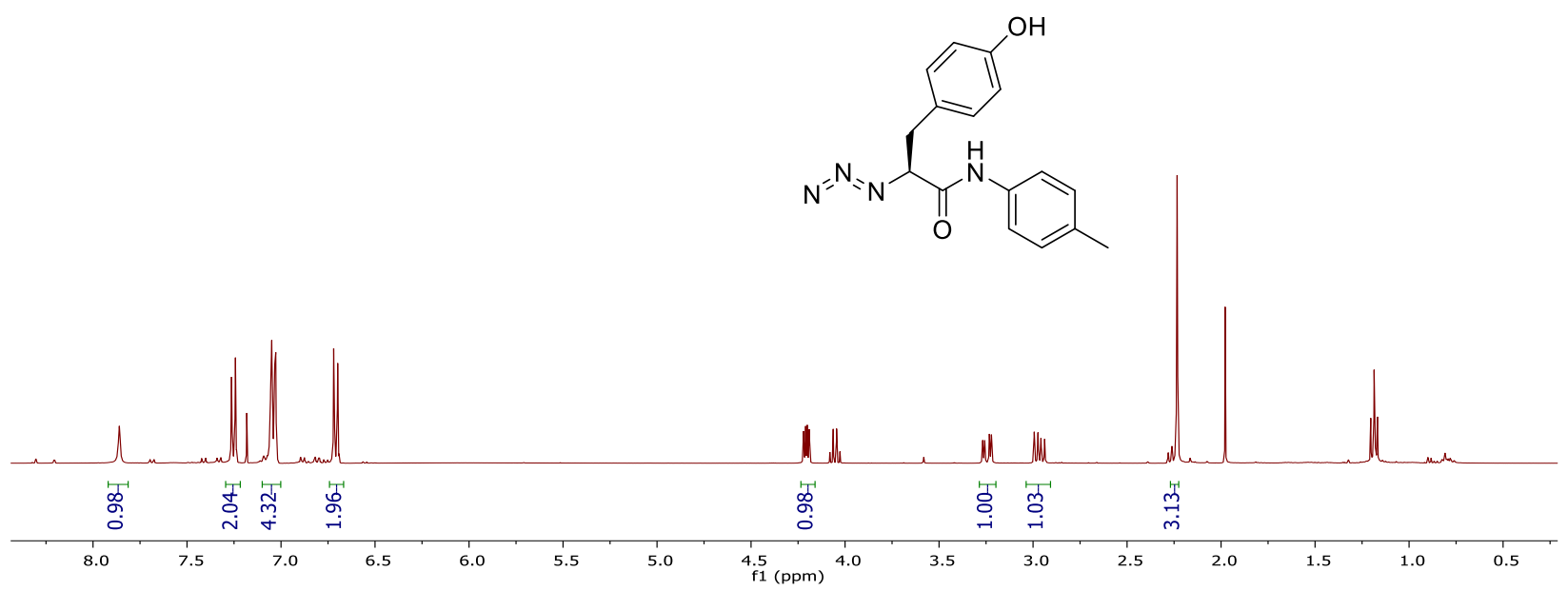


${ }^{13} \mathrm{C}\left\{{ }^{1} \mathrm{H}\right\}$ NMR (101 MHz, Chloroform- $d$ ) spectra of $\mathbf{5 f}$

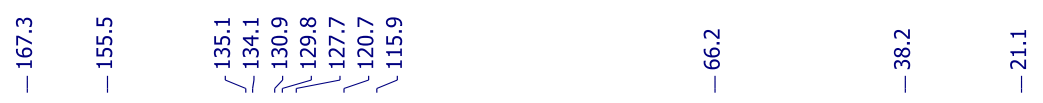
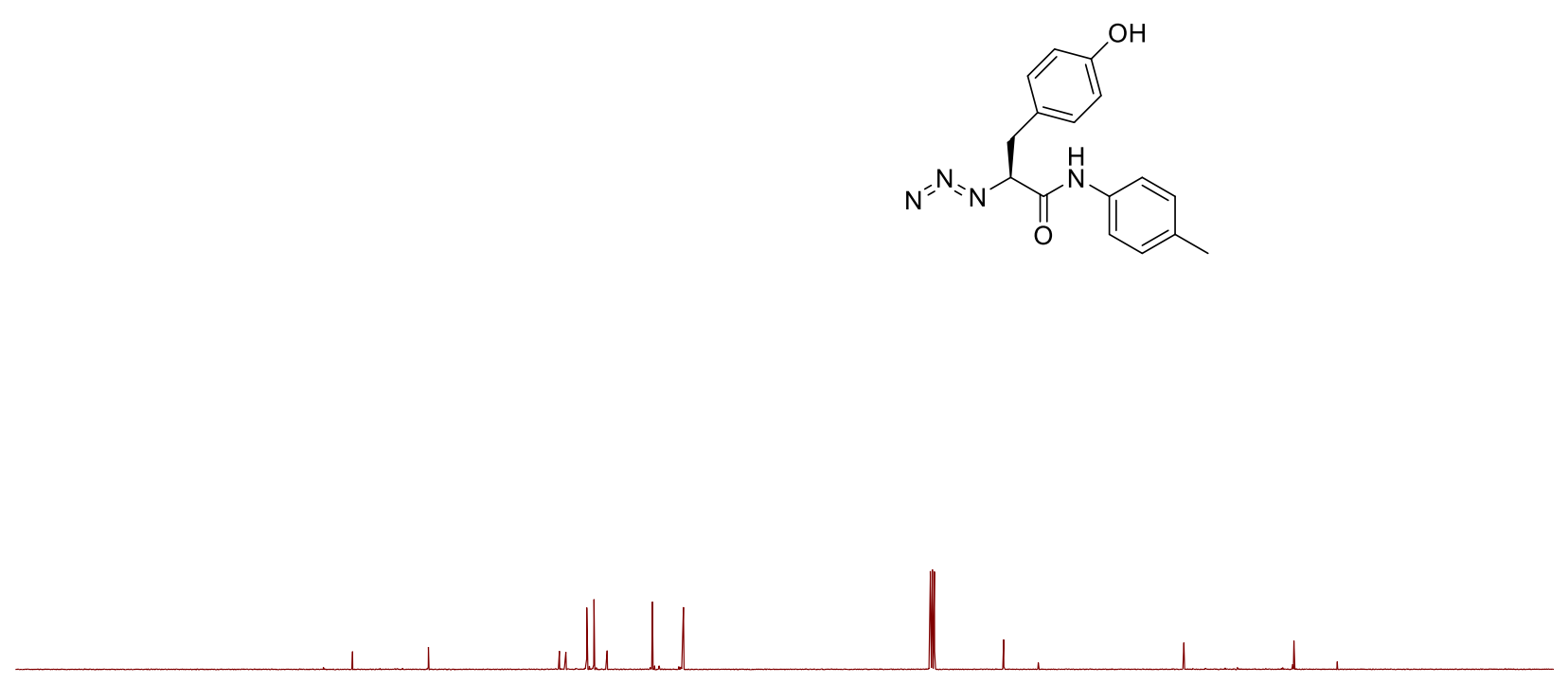

$\begin{array}{llllllllllllllllllllllll}1 & 210 & 200 & 190 & 180 & 170 & 160 & 150 & 140 & 130 & 120 & 110 & 100 & 90 & 80 & 70 & 60 & 50 & 40 & 30 & 20 & 10 & 0 & -10\end{array}$

\section{HRMS of $5 f$}

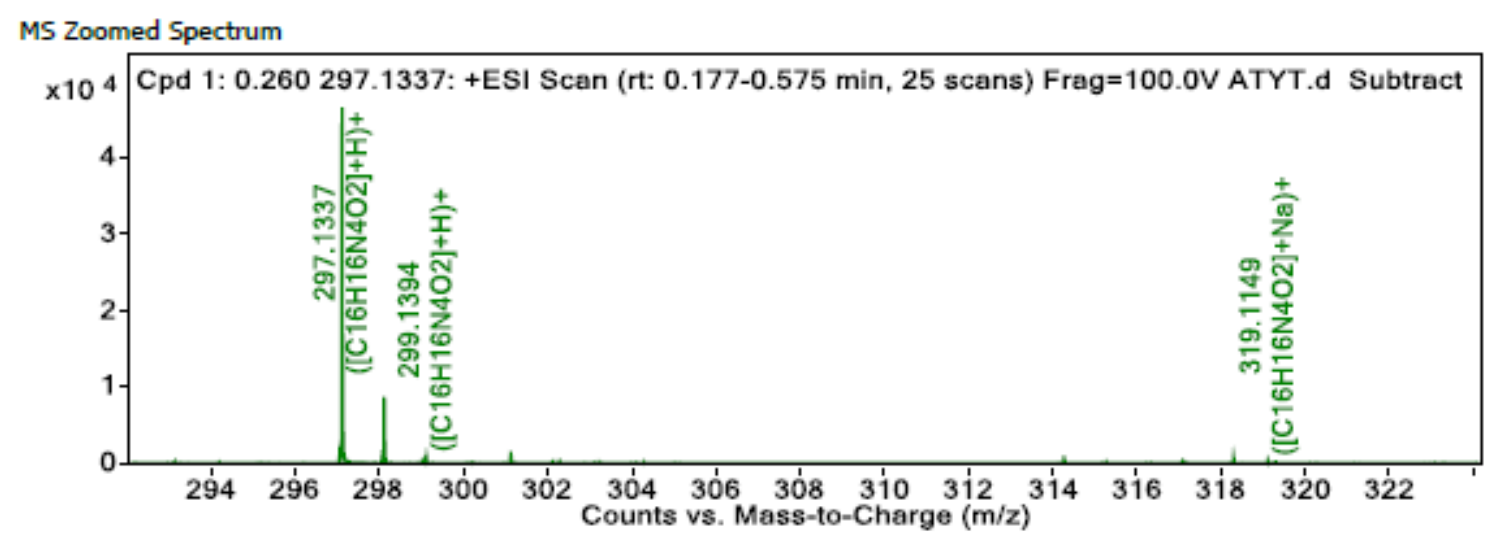


${ }^{1} \mathrm{H}$ NMR (400 MHz, Chloroform- $d$ ) spectra of $5 \mathbf{g}$

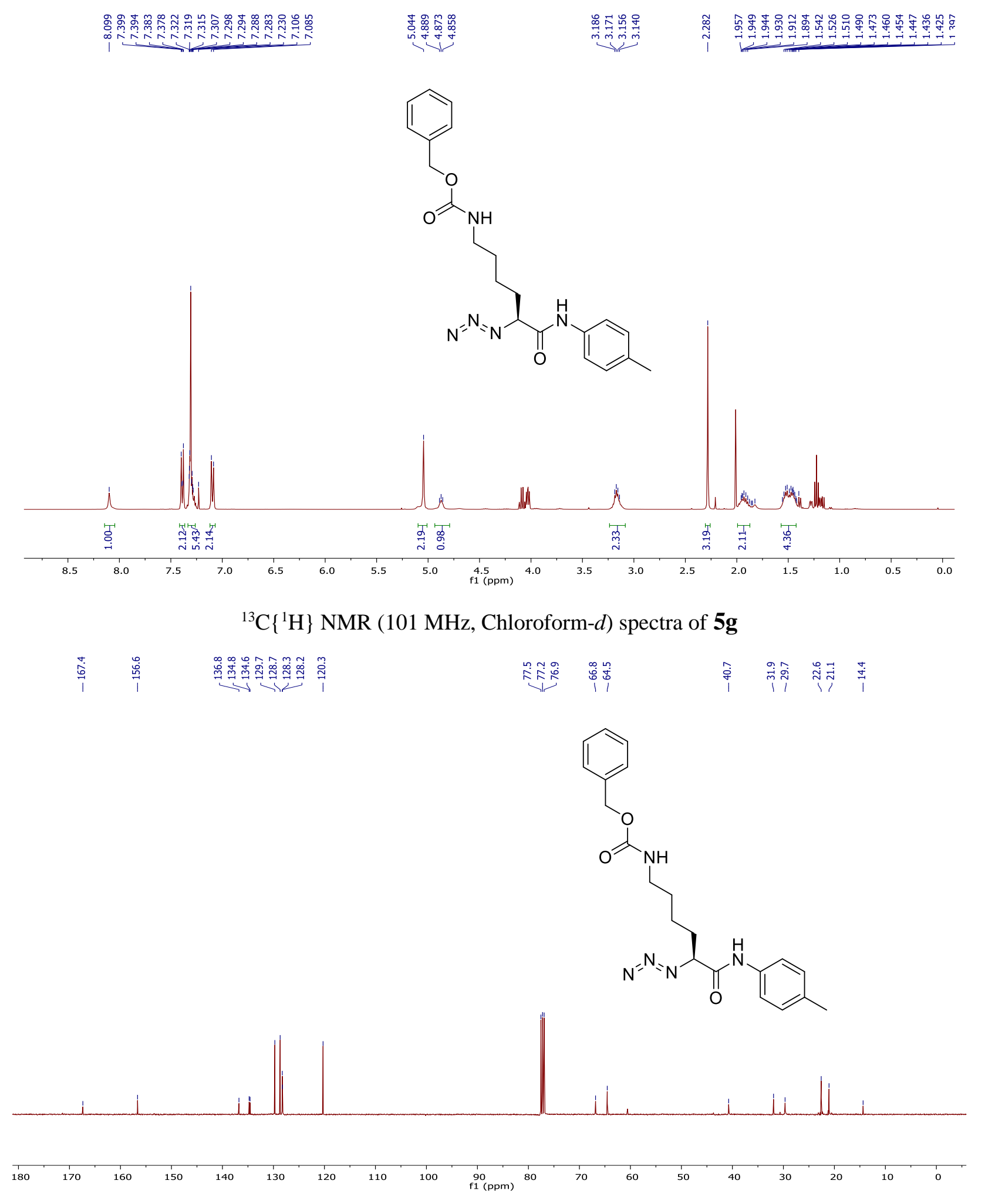




\section{HRMS of $\mathbf{5 g}$}

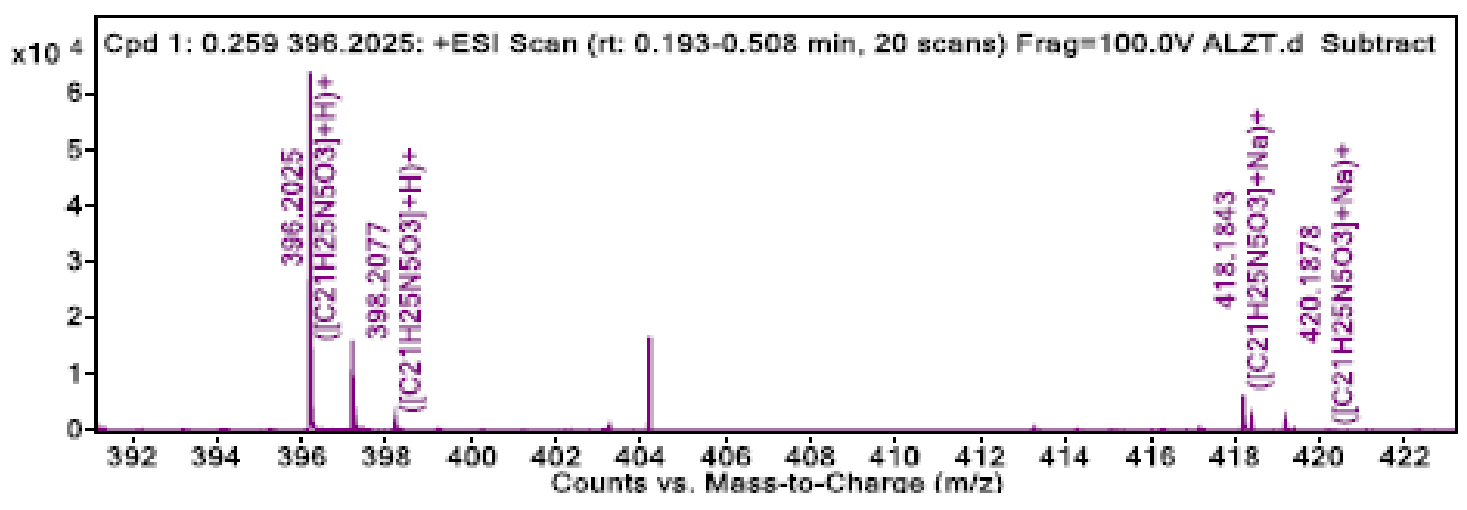

${ }^{1} \mathrm{H}$ NMR (400 MHz, Chloroform- $d$ ) spectra of $\mathbf{5 h}$

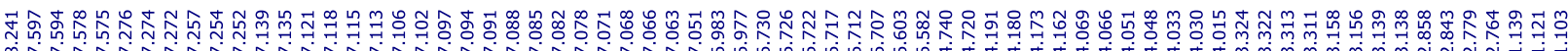

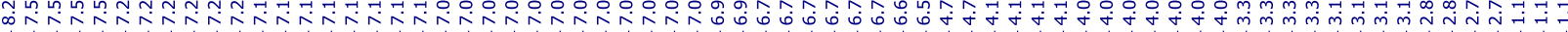<smiles></smiles>

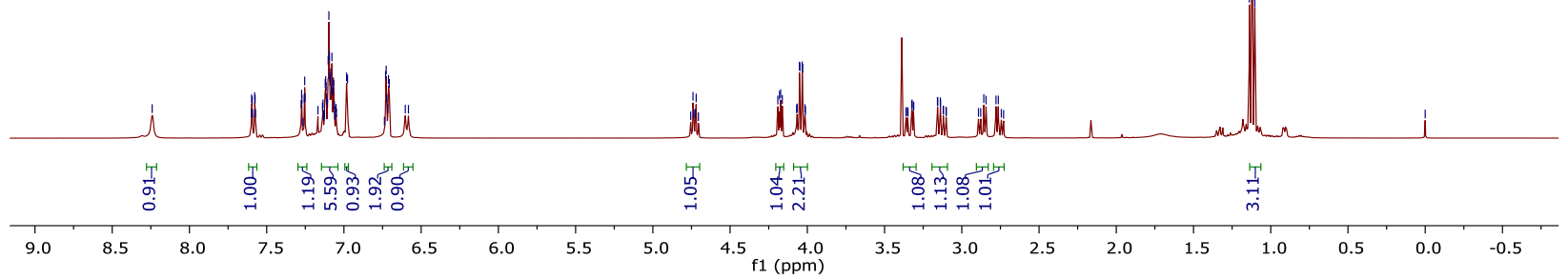


${ }^{13} \mathrm{C}\left\{{ }^{1} \mathrm{H}\right\}$ NMR (101 MHz, Chloroform- $d$ ) spectra of $\mathbf{5 h}$
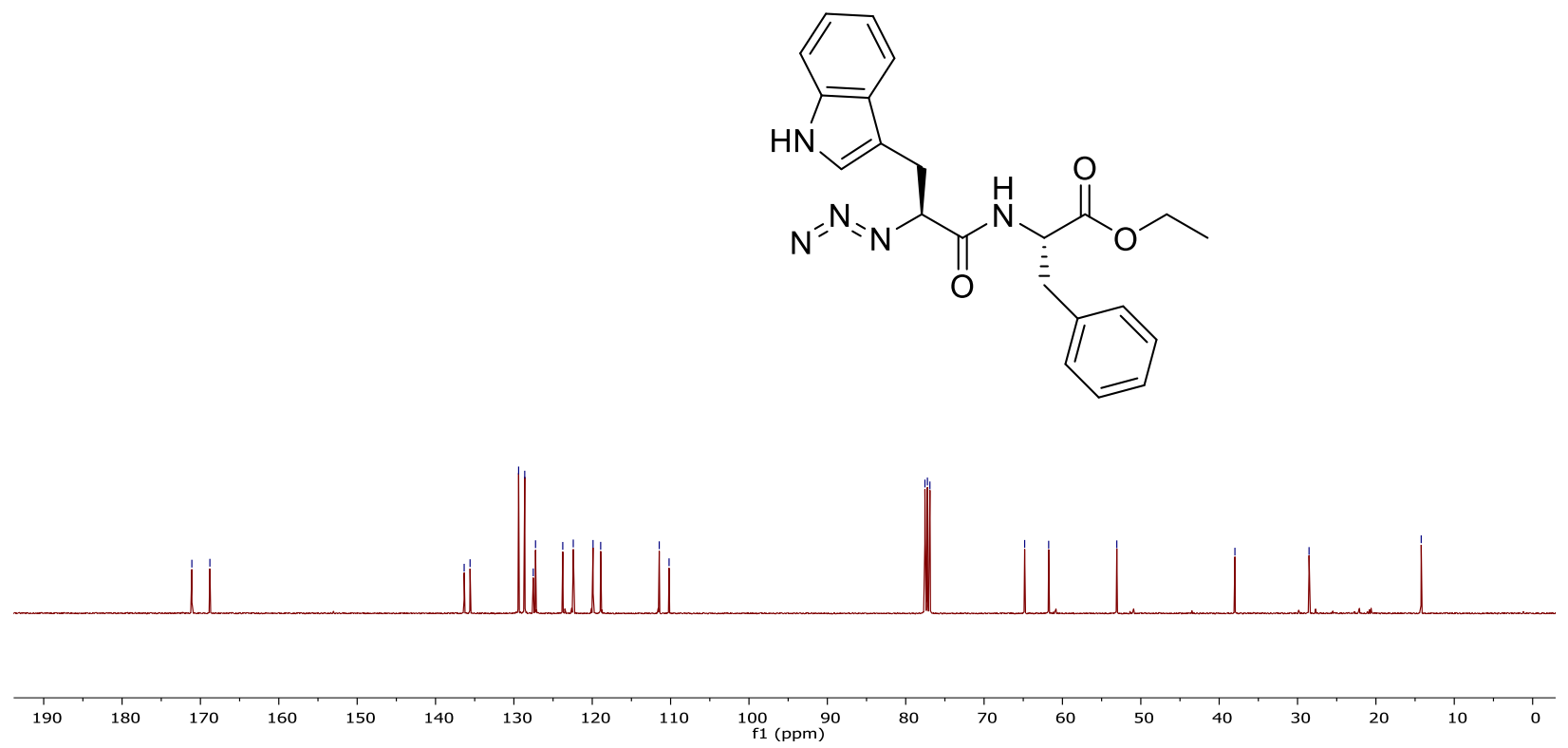

HRMS of $\mathbf{5 h}$

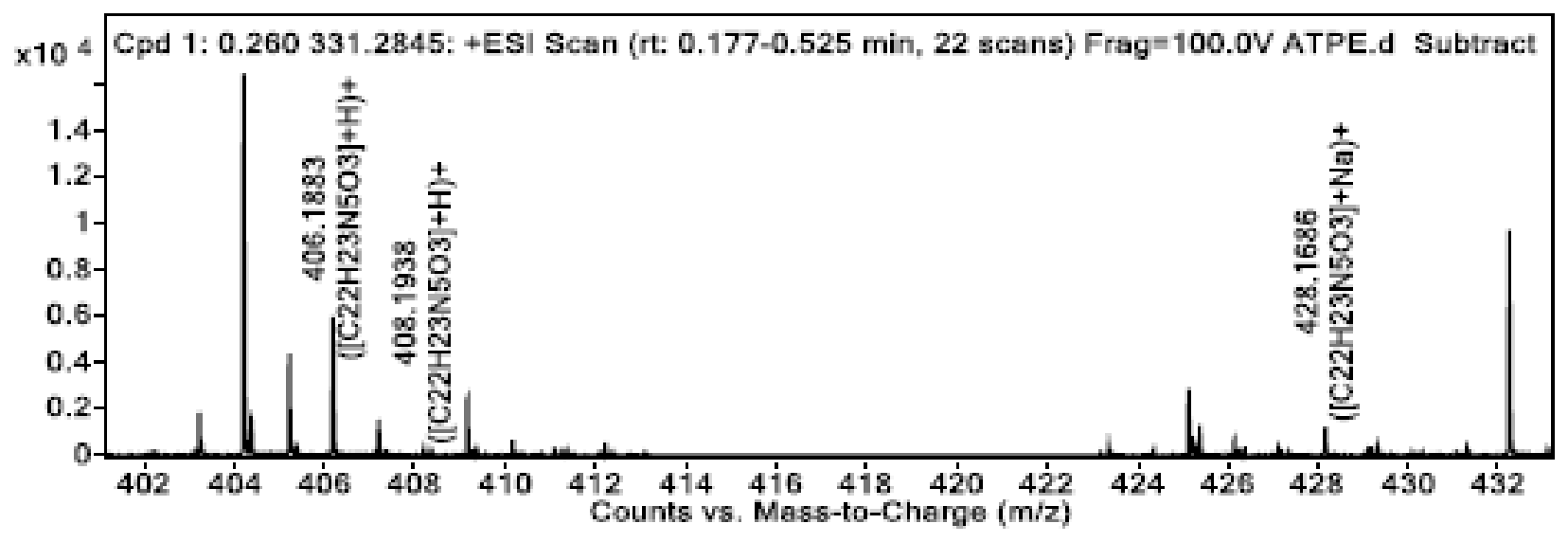


${ }^{1} \mathrm{H}$ NMR (400 MHz, Chloroform- $d$ ) spectra of $\mathbf{5 i}$

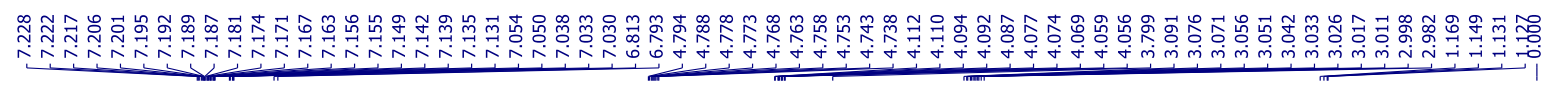

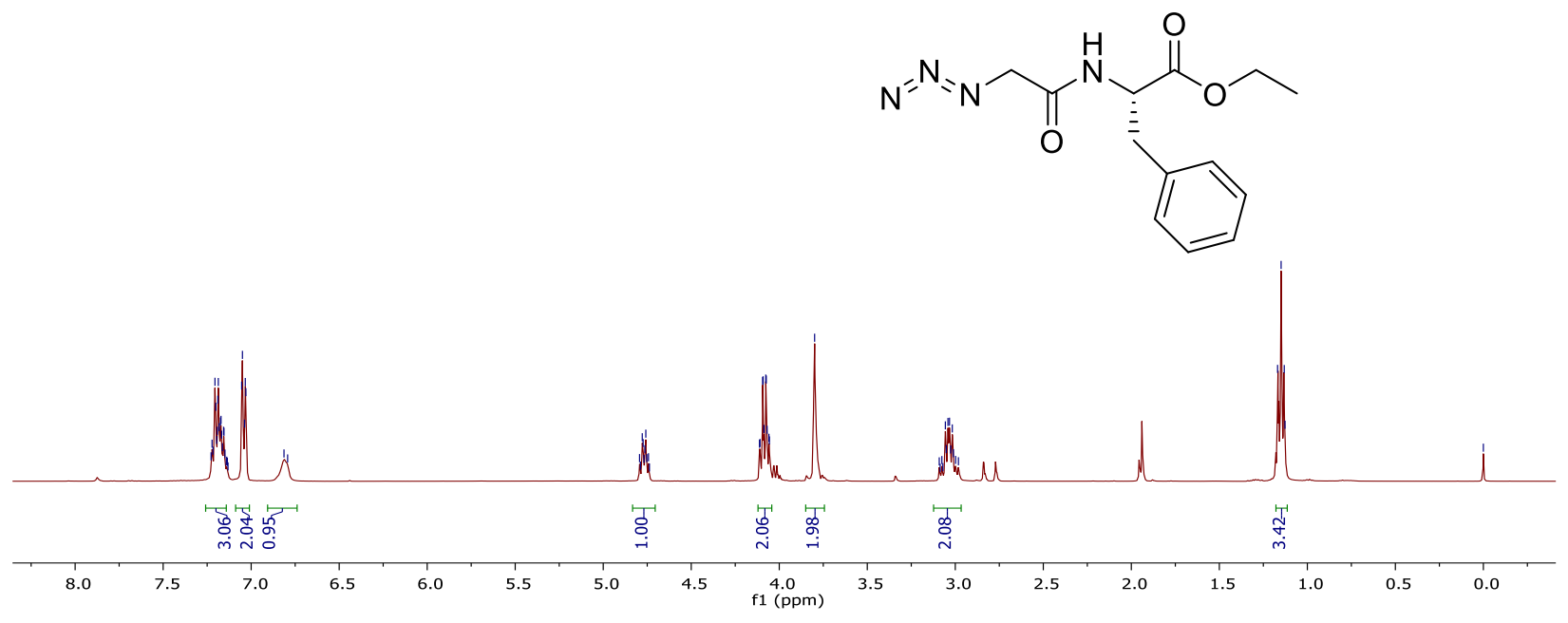

${ }^{13} \mathrm{C}\left\{{ }^{1} \mathrm{H}\right\}$ NMR (101 MHz, Chloroform- $d$ ) spectra of $\mathbf{5 i}$

\begin{tabular}{|c|}
\hline 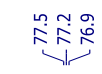 \\
\hline
\end{tabular}
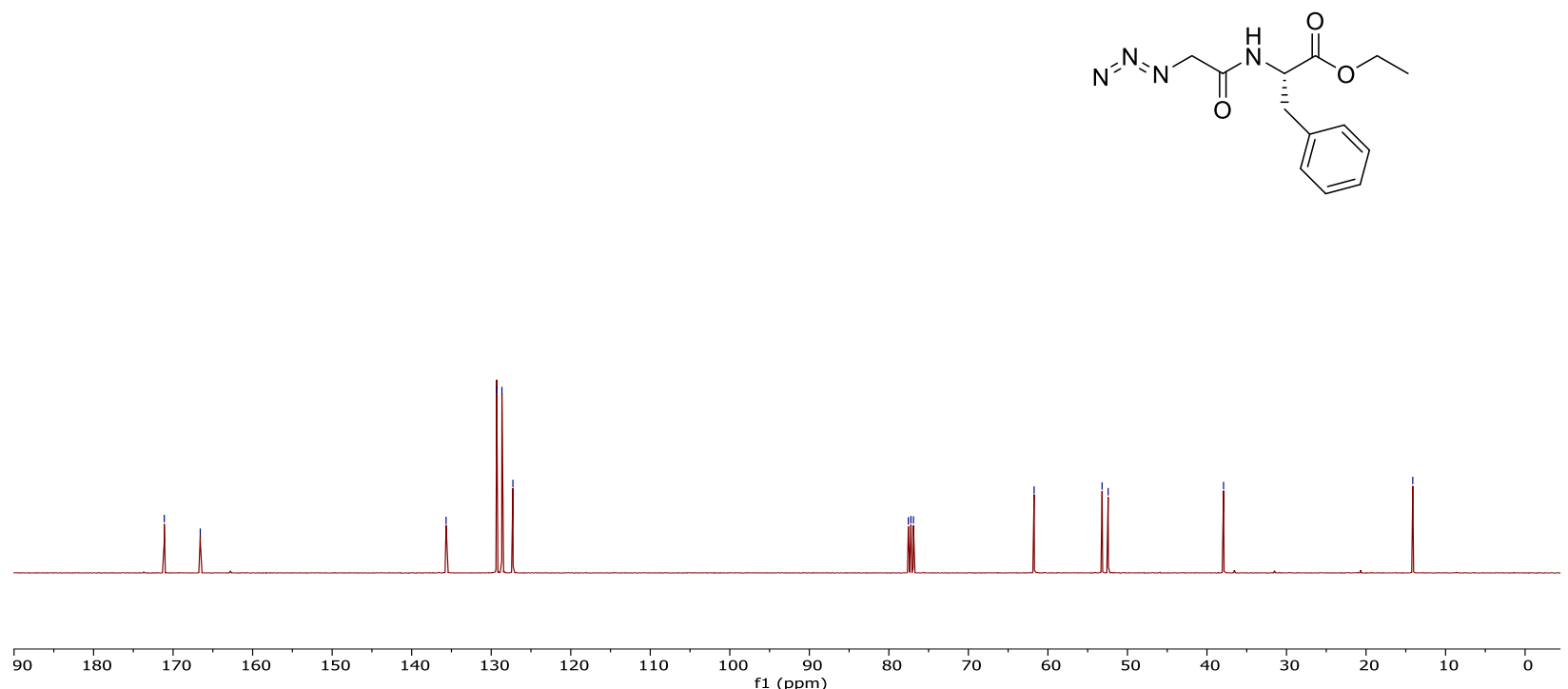
3. Original NMR and HRMS spectra of $\mathbf{6 a a - 6 i ' h ~}$

${ }^{1} \mathrm{H}$ NMR (400 MHz, DMSO- $d_{6}$ ) spectra of 6aa

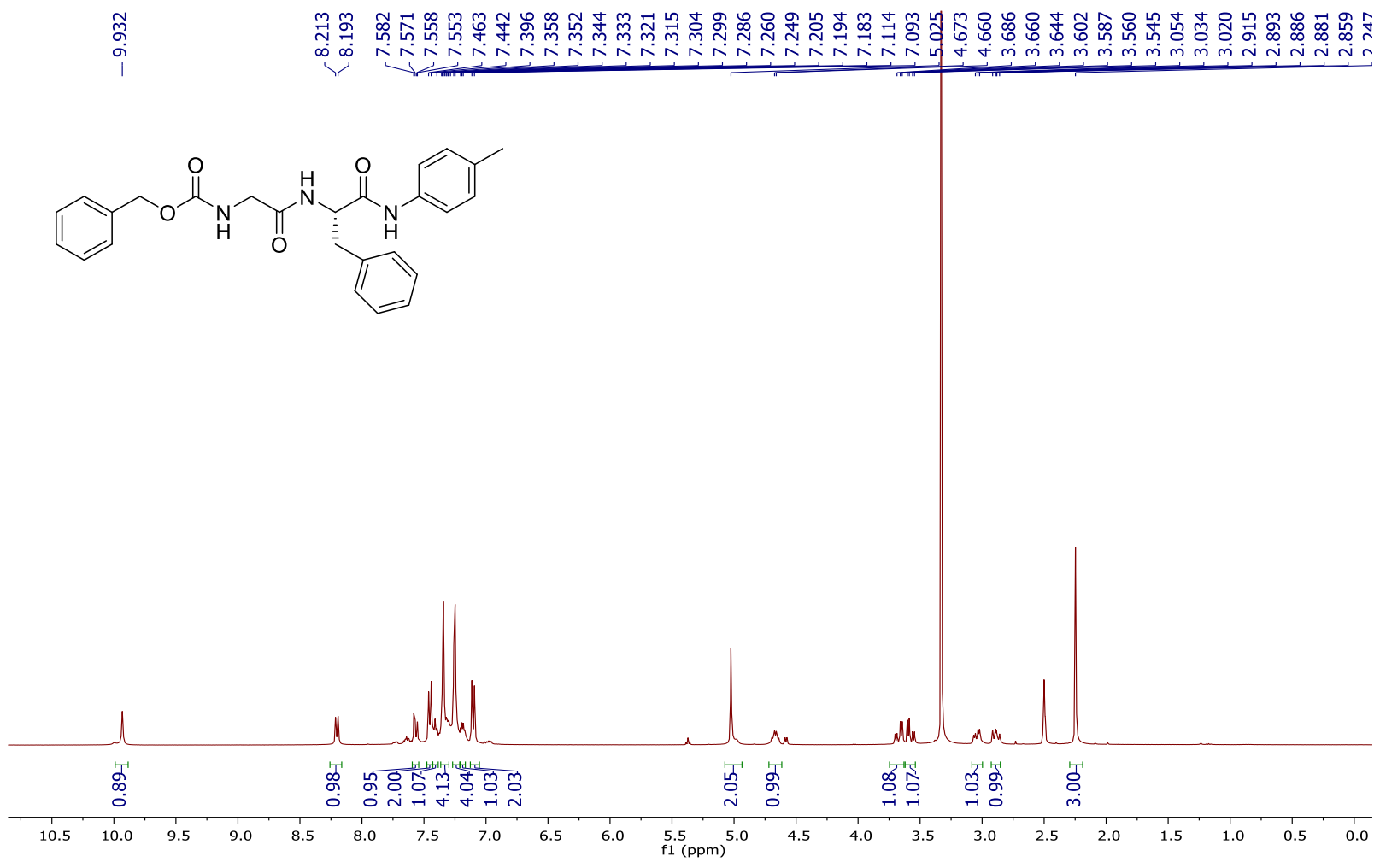

${ }^{13} \mathrm{C}\left\{{ }^{1} \mathrm{H}\right\}$ NMR (101 MHz, DMSO- $\left.d_{6}\right)$ spectra of 6aa

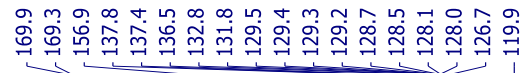

照<smiles>Cc1ccc(NC(=O)[C@H](Cc2ccccc2)NC(=O)CNC(=O)OCc2ccccc2)cc1</smiles>
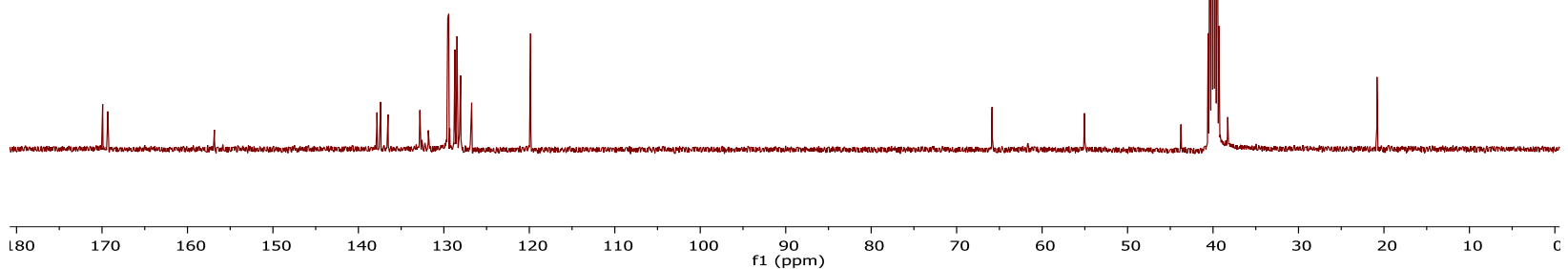


\section{HRMS of 6aa}

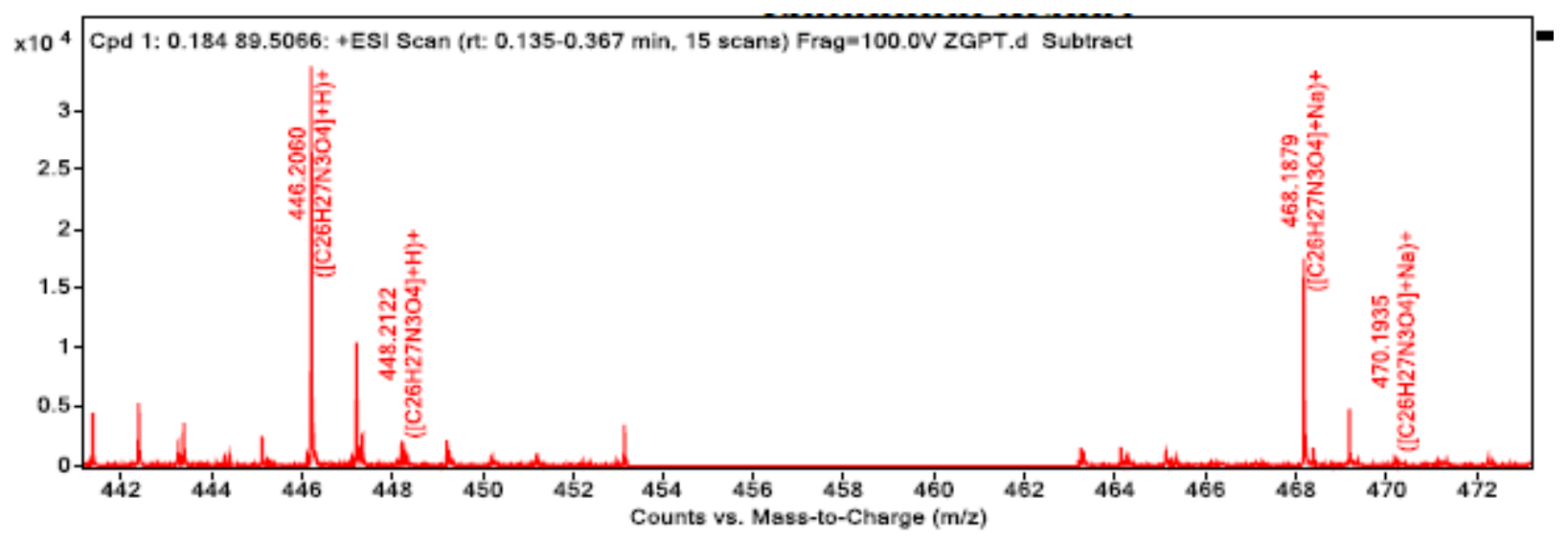

${ }^{1} \mathrm{H}$ NMR (400 MHz, DMSO-d $d_{6}$ ) spectra of $\mathbf{6 a b}$

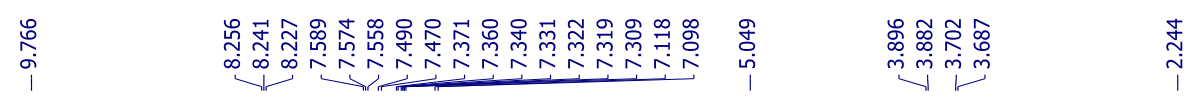<smiles>Cc1ccc(NC(=O)CNC(=O)CNC(=O)OCc2ccccc2)cc1</smiles>

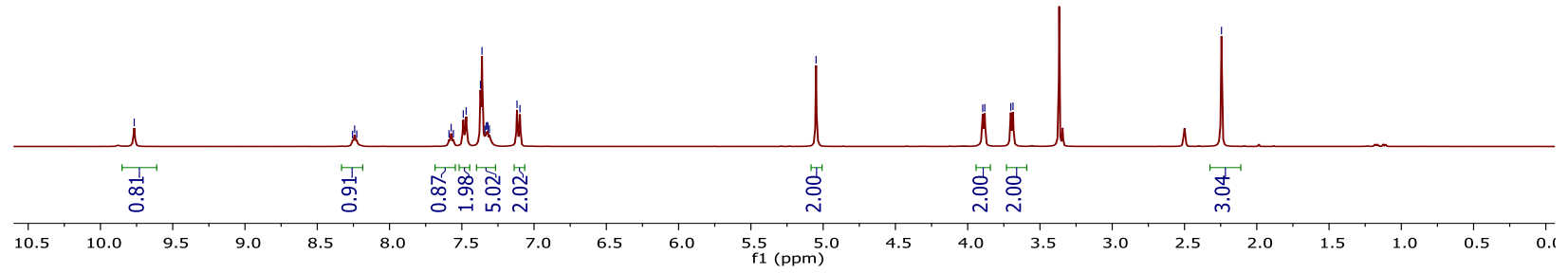




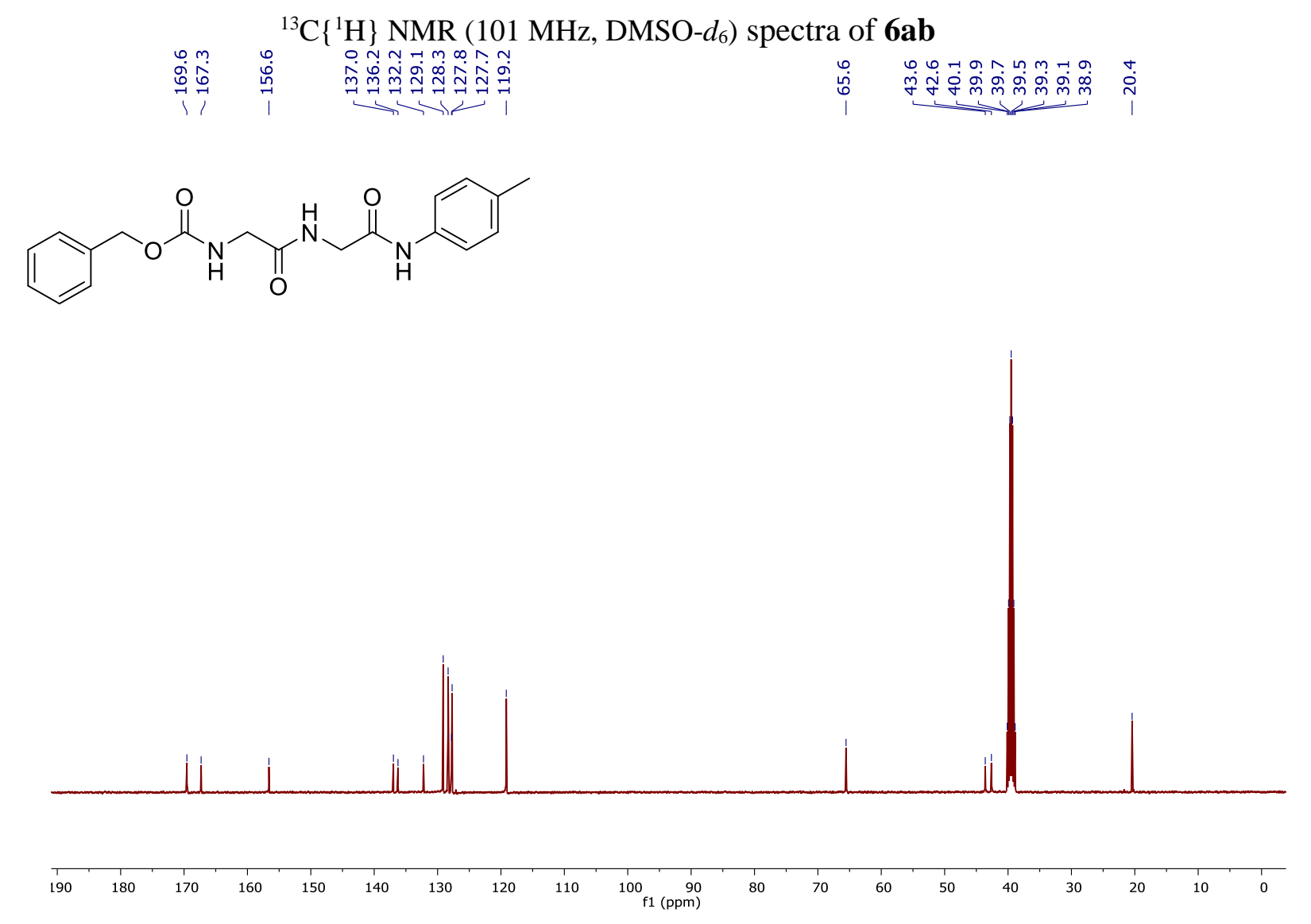

\section{HRMS of 6ab}

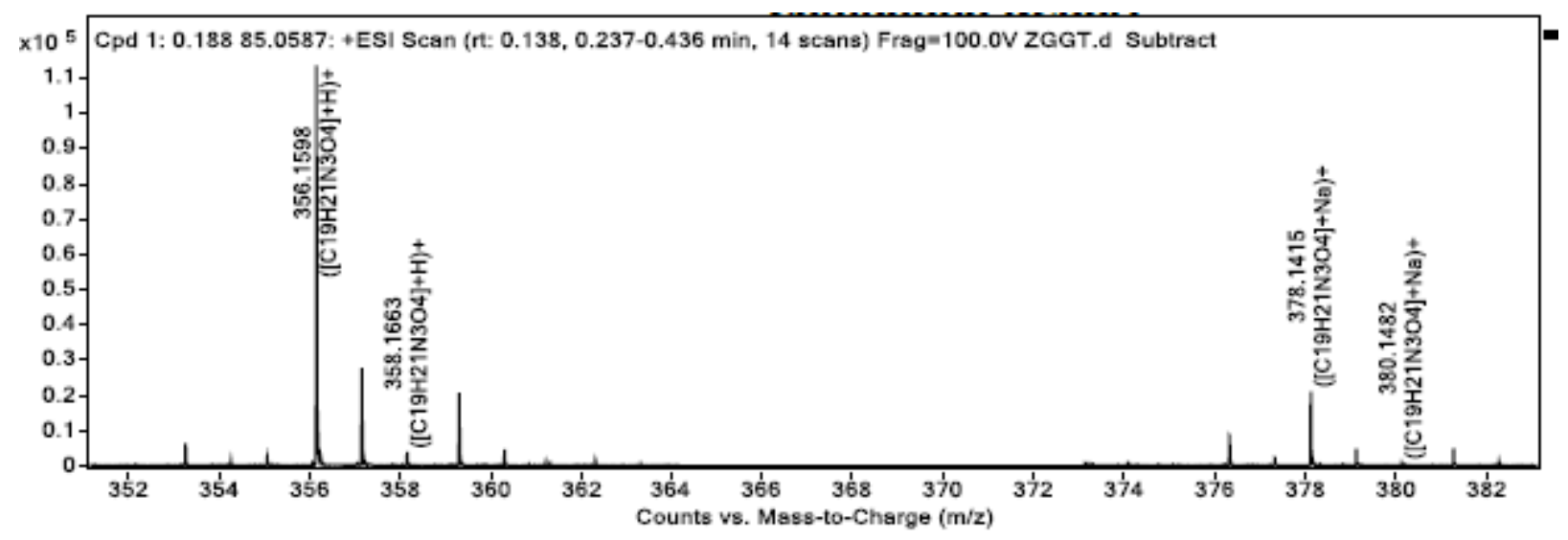


${ }^{1} \mathrm{H}$ NMR (400 MHz, DMSO- $d_{6}$ ) spectra of $\mathbf{6 a c}$

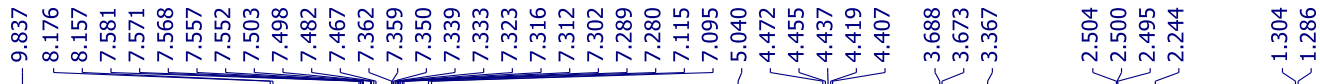<smiles>Cc1ccc(NC(=O)[C@H](C)NC(=O)CNC(=O)OCc2ccccc2)cc1</smiles>

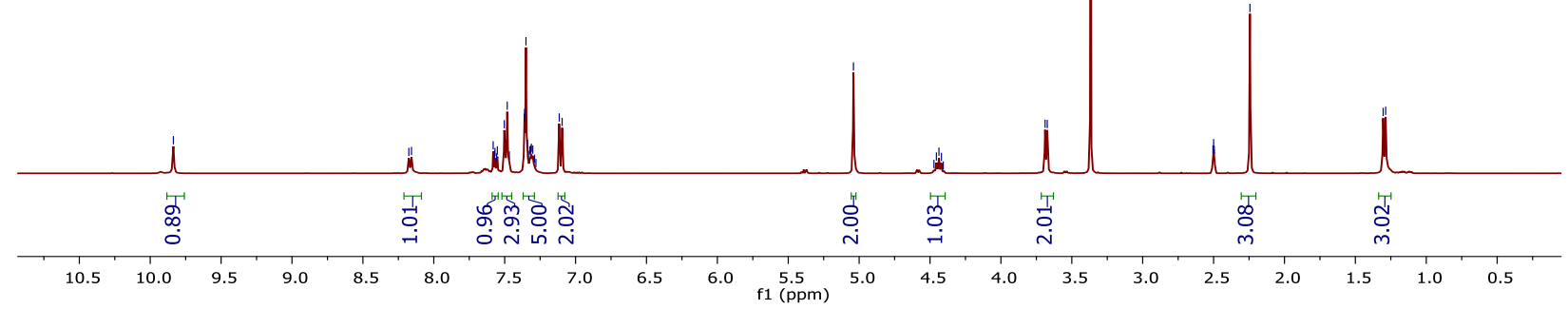

${ }^{13} \mathrm{C}\left\{{ }^{1} \mathrm{H}\right\}$ NMR (101 MHz, DMSO- $\left.d_{6}\right)$ spectra of $6 \mathbf{a c}$

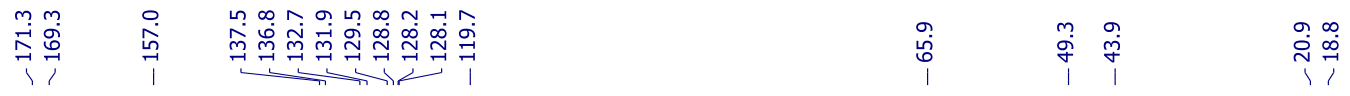<smiles>Cc1ccc(NC(=O)[C@H](C)NC(=O)CNC(=O)OCc2ccccc2)cc1</smiles>
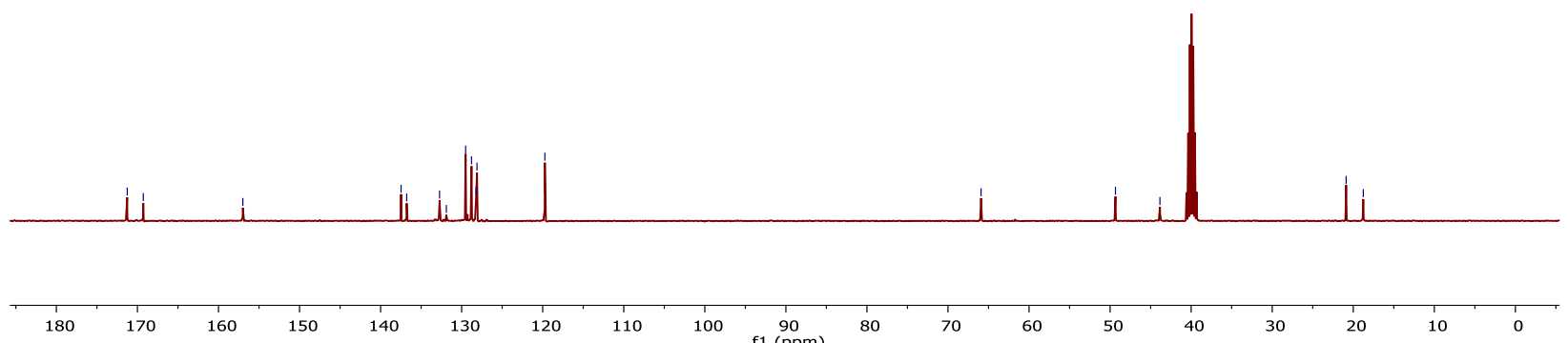


\section{HRMS of 6 ac}

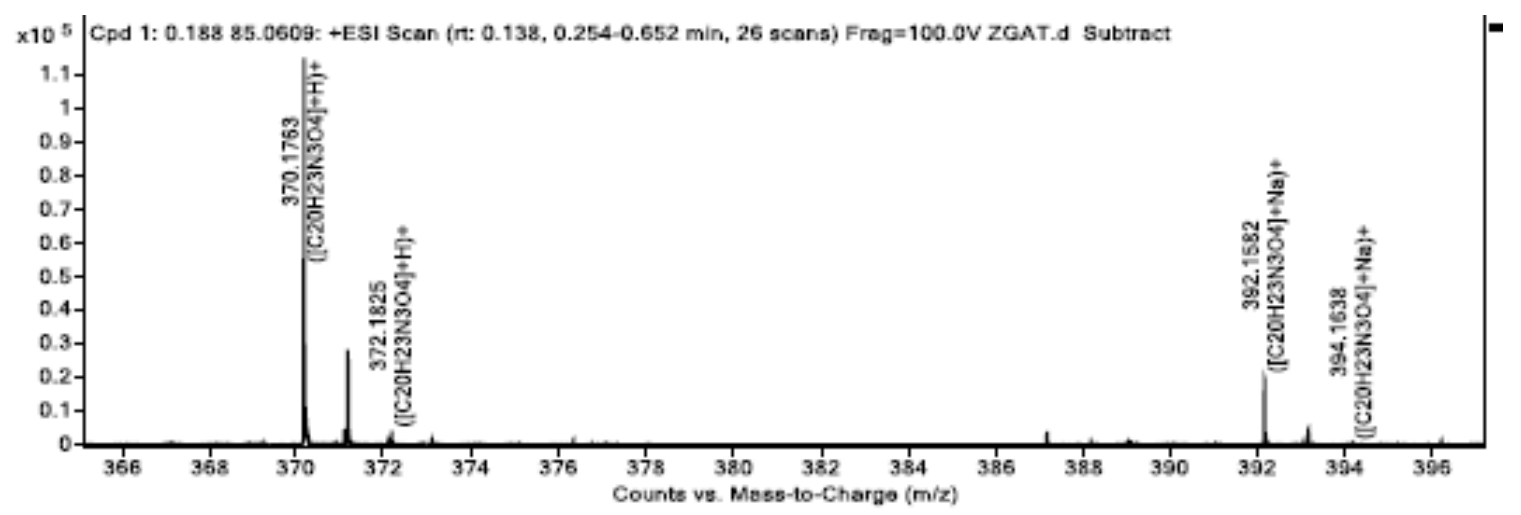

${ }^{1} \mathrm{H}$ NMR (400 MHz, Chloroform- $d$ ) spectra of $6 \mathbf{a d}$

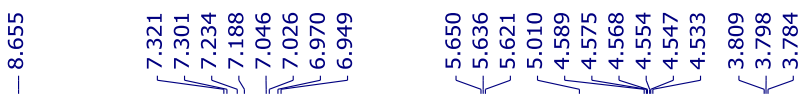

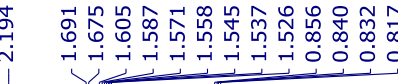
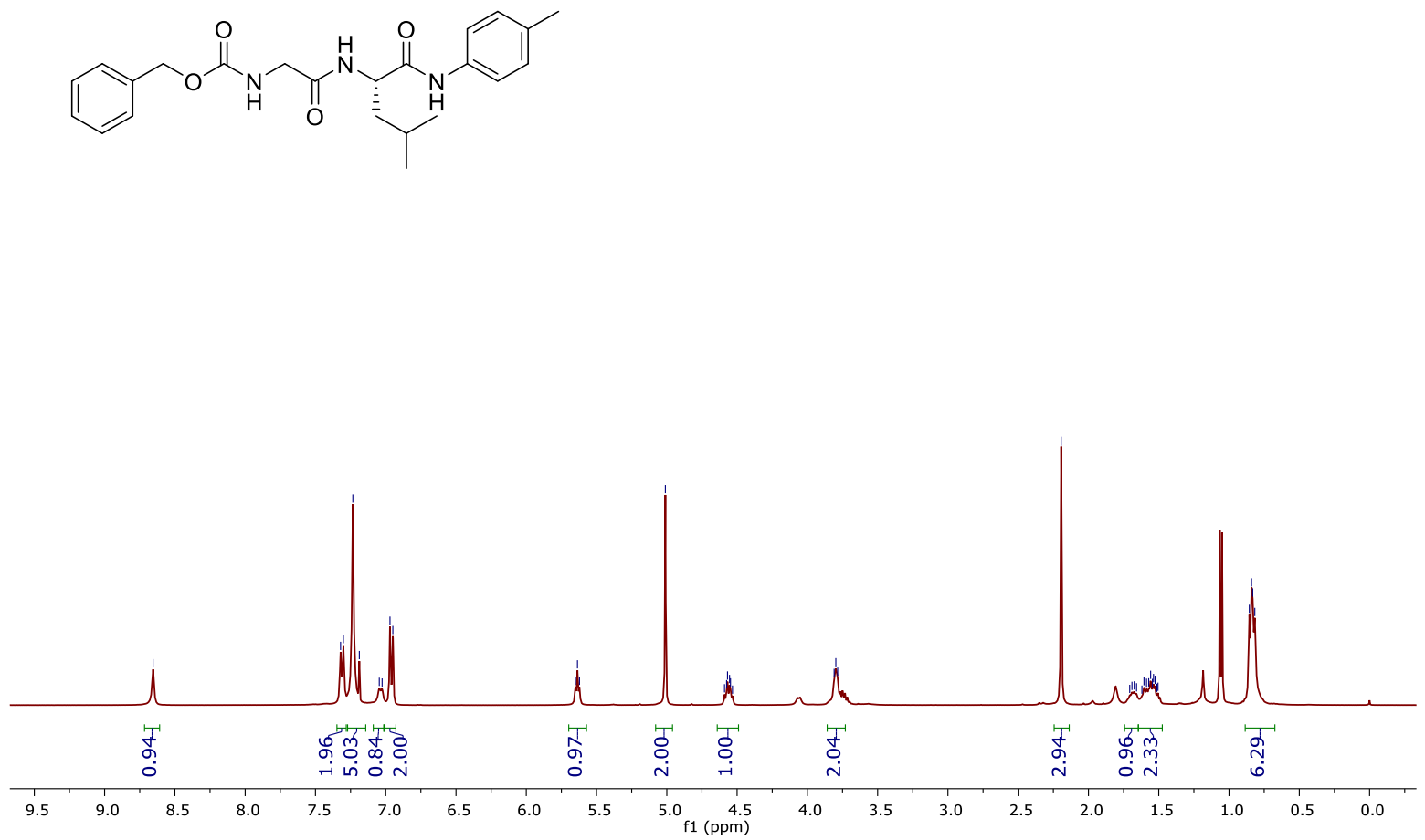


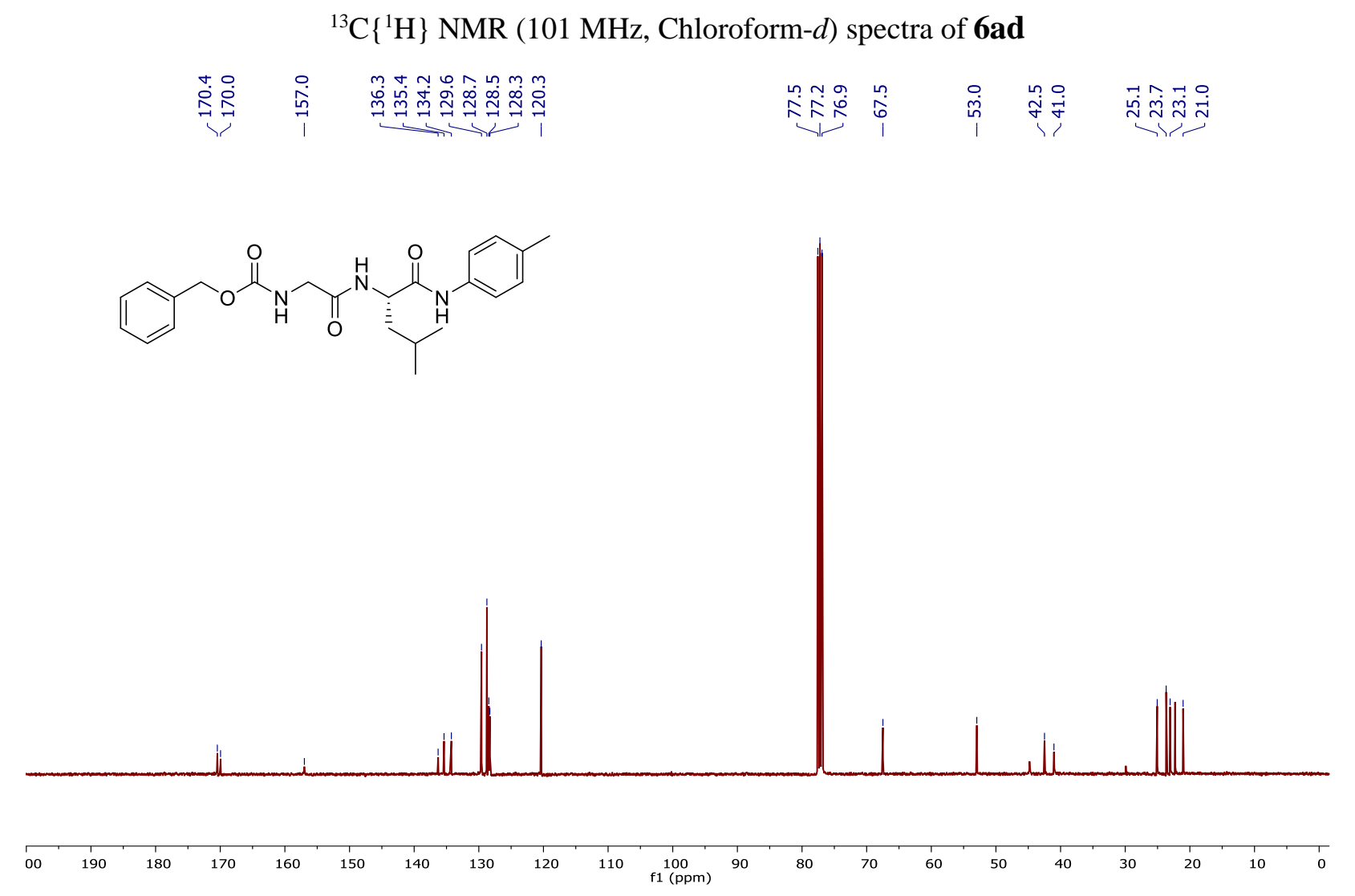

\section{HRMS of 6ad}

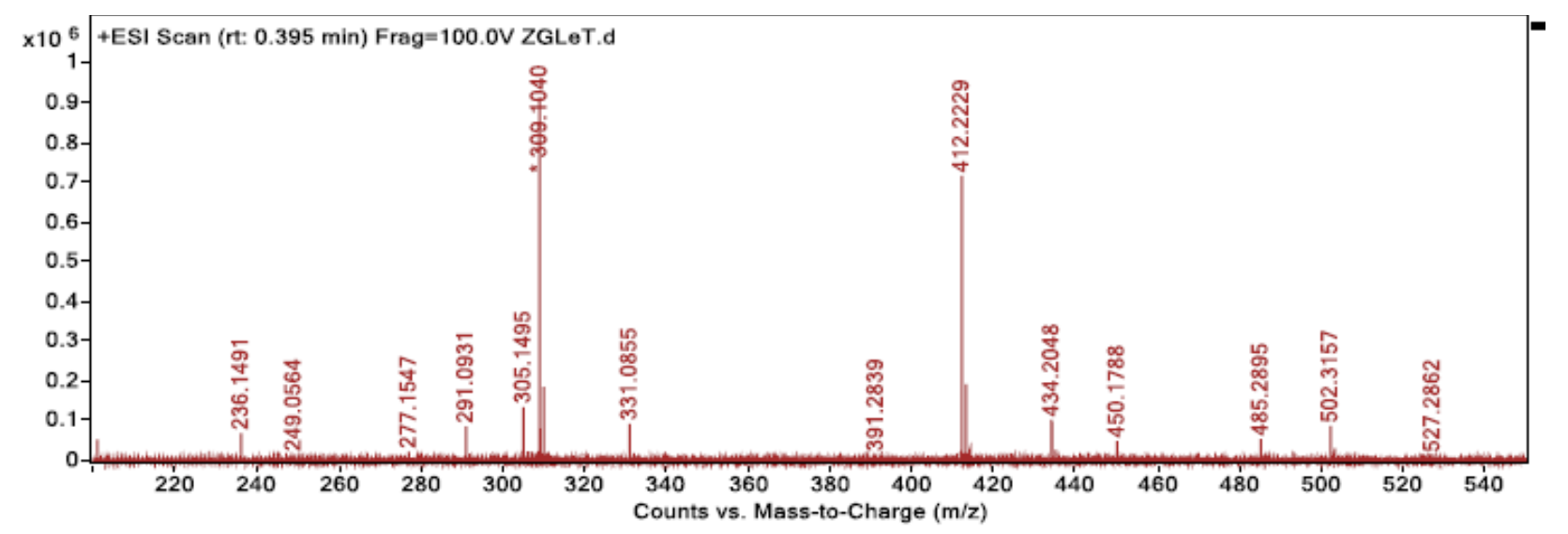


${ }^{1} \mathrm{H}$ NMR (400 MHz, DMSO- $d_{6}$ ) spectra of $\mathbf{6 a e}$

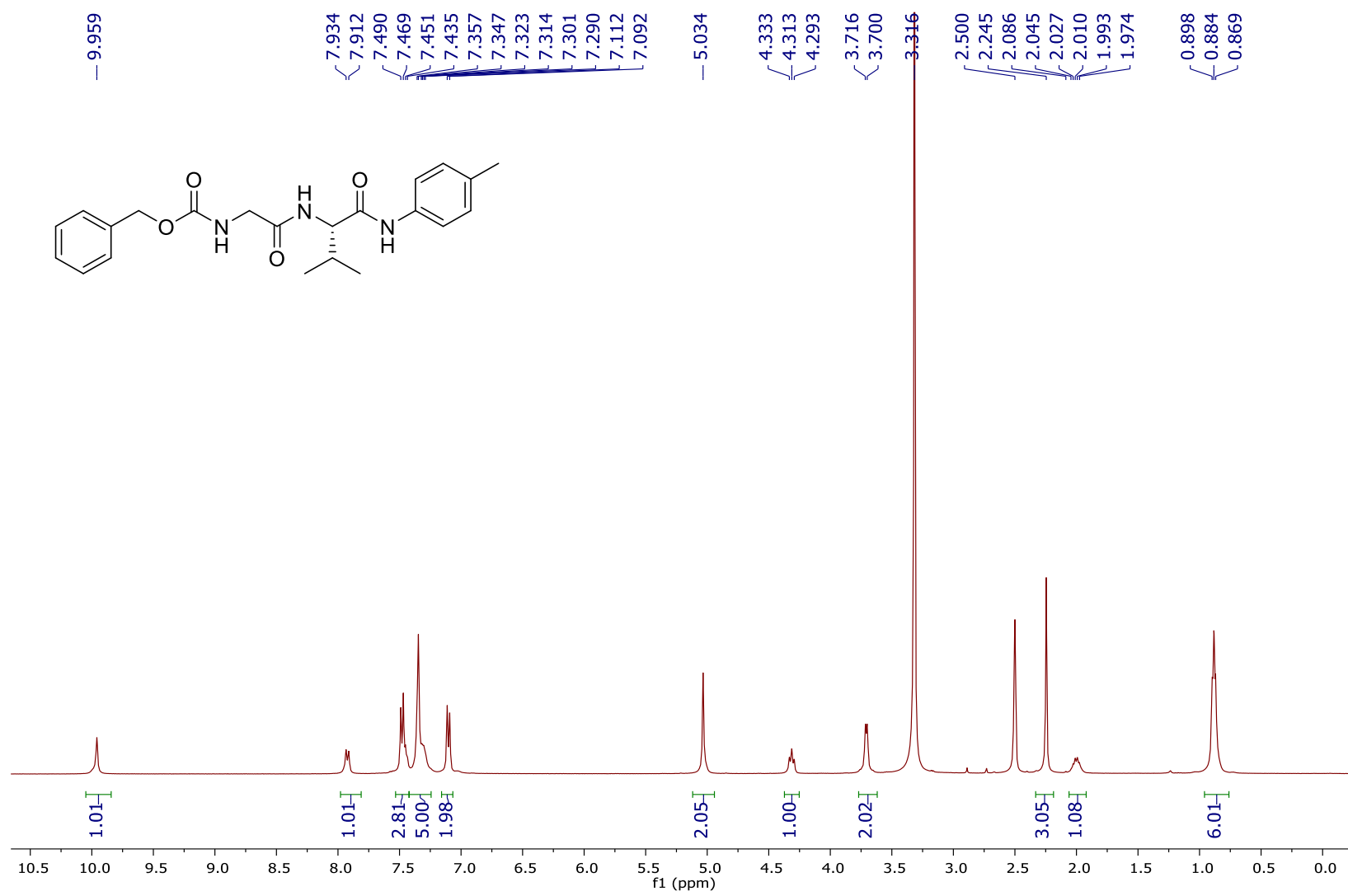

${ }^{13} \mathrm{C}\left\{{ }^{1} \mathrm{H}\right\}$ NMR (101 MHz, DMSO- $\left.d_{6}\right)$ spectra of $6 \mathbf{a e}$
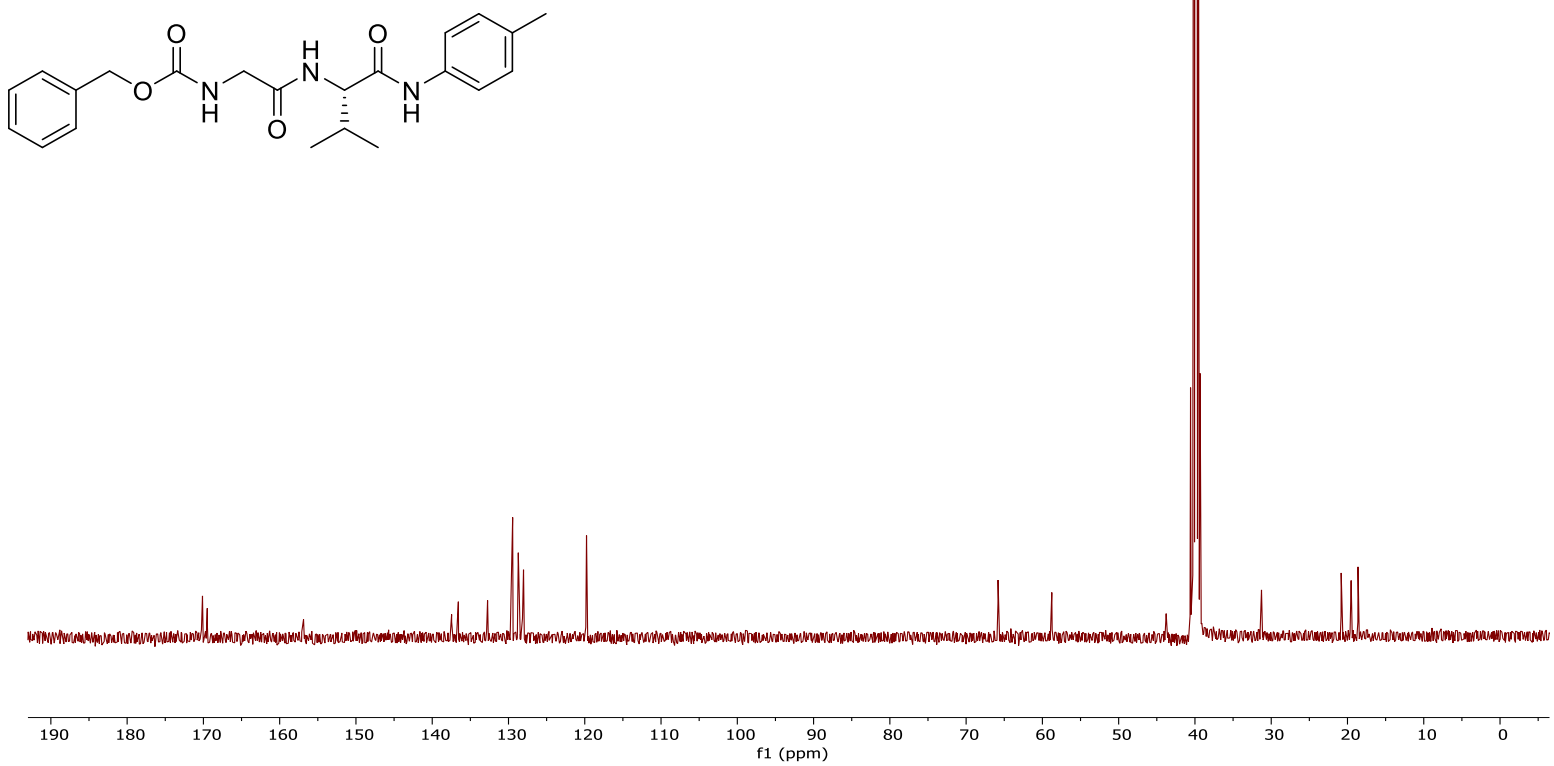


\section{HRMS of 6ae}

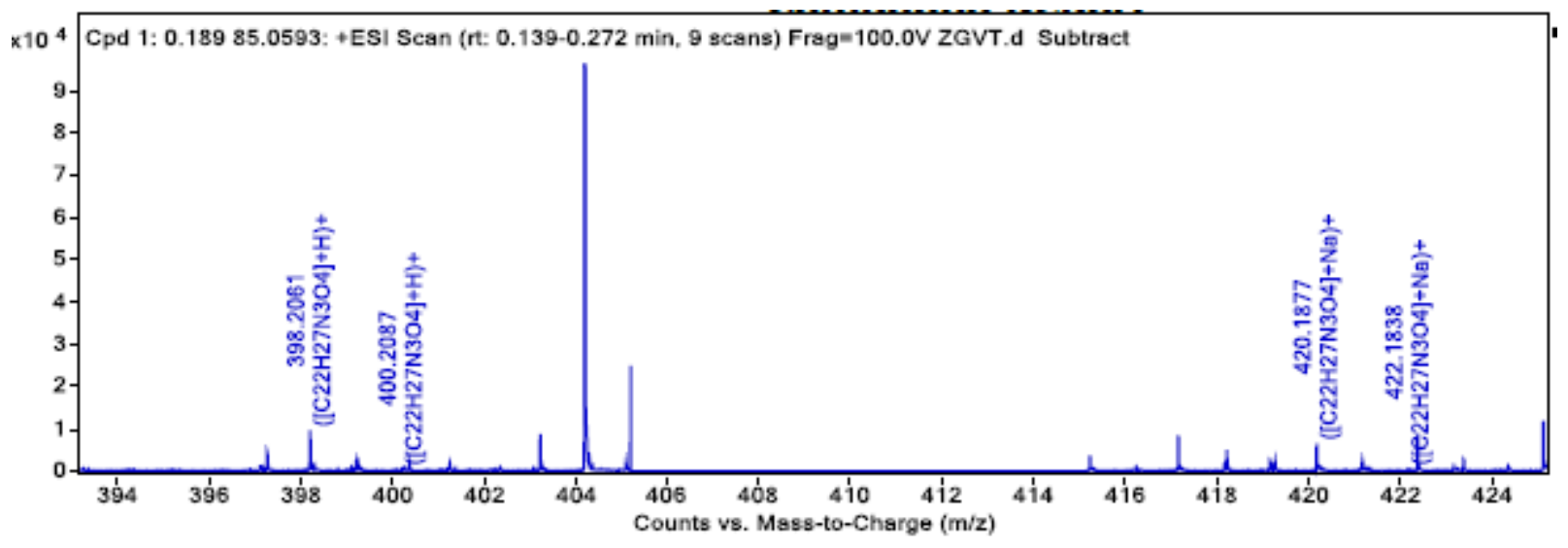

${ }^{1} \mathrm{H}$ NMR (400 MHz, DMSO- $d_{6}$ ) spectra of $\mathbf{6 a f}$

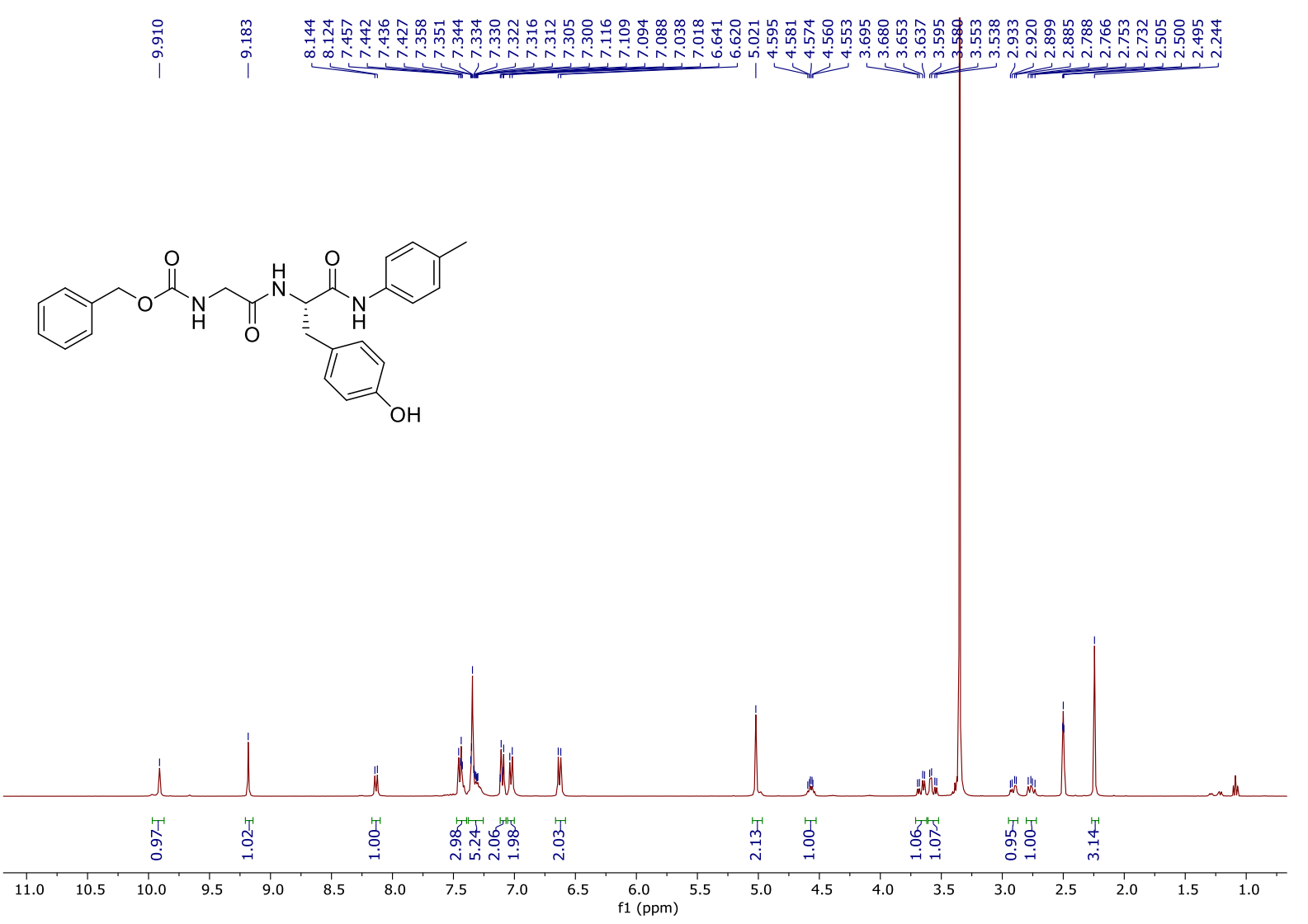


${ }^{13} \mathrm{C}\left\{{ }^{1} \mathrm{H}\right\}$ NMR (101 MHz, DMSO- $\left.d_{6}\right)$ spectra of $\mathbf{6 a f}$

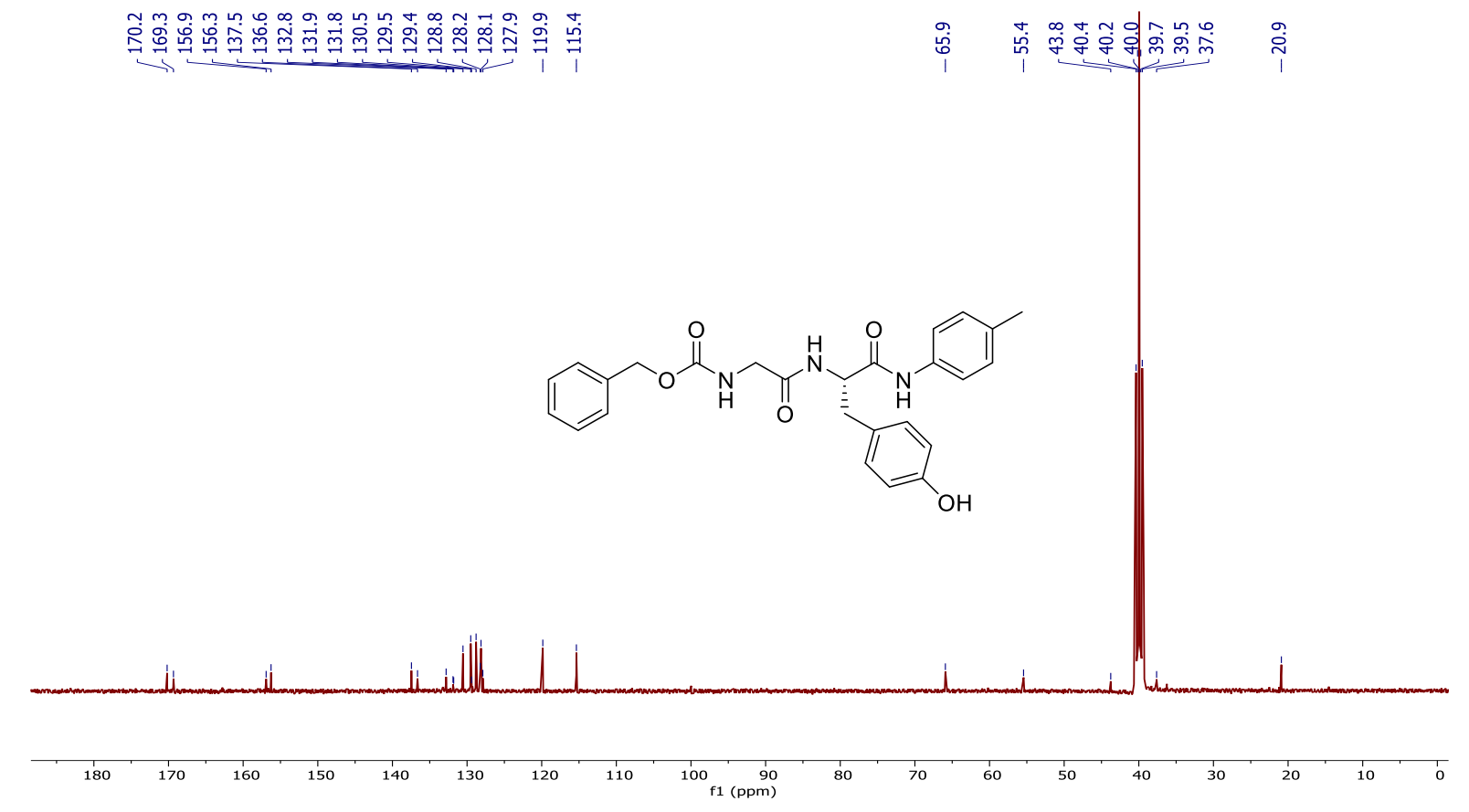

HRMS of 6 af

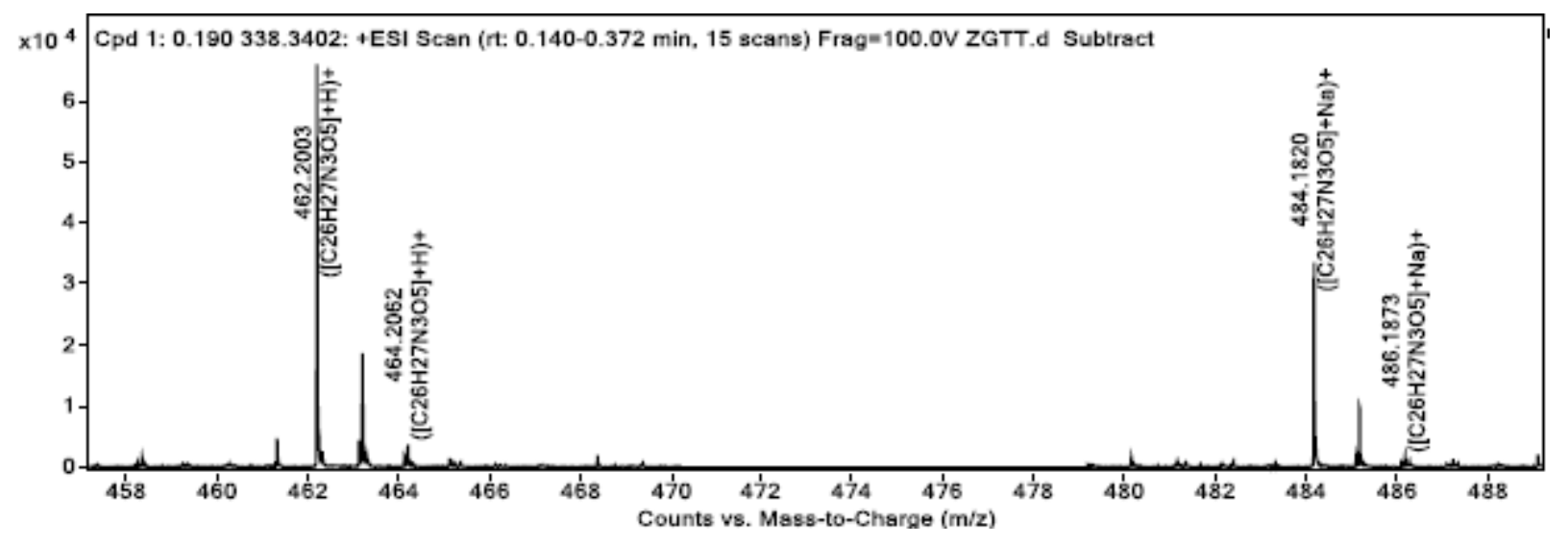




\section{${ }^{1} \mathrm{H}$ NMR (400 MHz, DMSO- $\left.d_{6}\right)$ spectra of $\mathbf{6 a g}$}

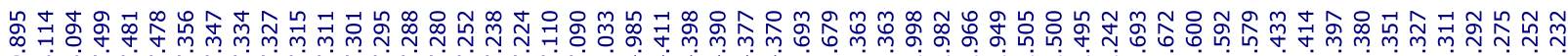
бં<smiles>Cc1ccc(NC(=O)C(CCCCNC(=O)OCc2ccccc2)NC(=O)CNC(=O)OCc2ccccc2)cc1</smiles>

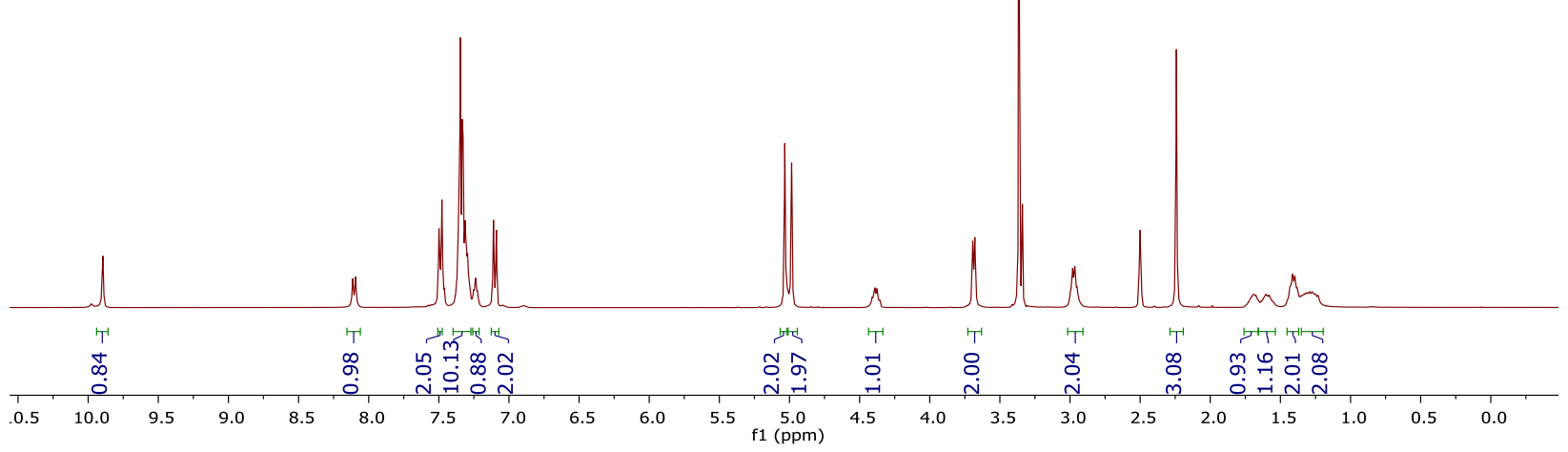

${ }^{13} \mathrm{C}\left\{{ }^{1} \mathrm{H}\right\}$ NMR (101 MHz, DMSO- $\left.d_{6}\right)$ spectra of $\mathbf{6 a g}$

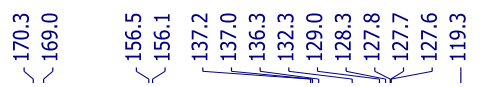

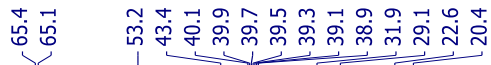<smiles>Cc1ccc(NC(=O)C(CCCCNC(=O)OCc2ccccc2)NC(=O)CNC(=O)OCc2ccccc2)cc1</smiles>

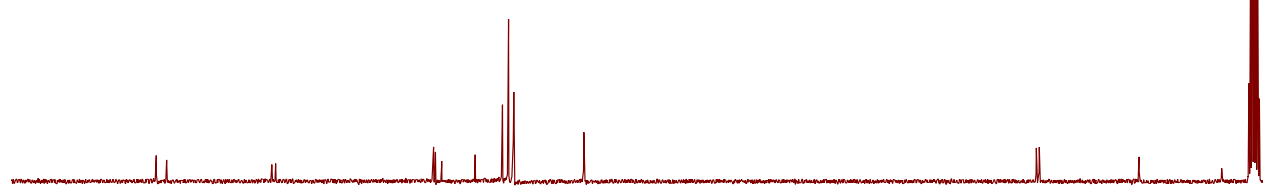




\section{HRMS of 6ag}

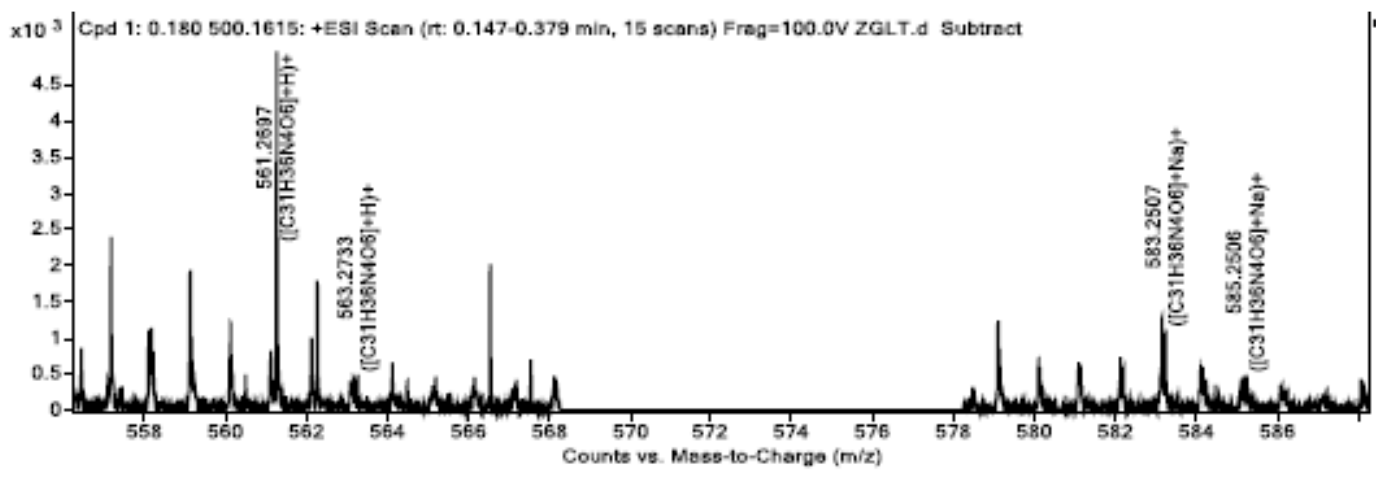

${ }^{1} \mathrm{H}$ NMR (400 MHz, DMSO- $d_{6}$ ) spectra of $\mathbf{6 b c}$

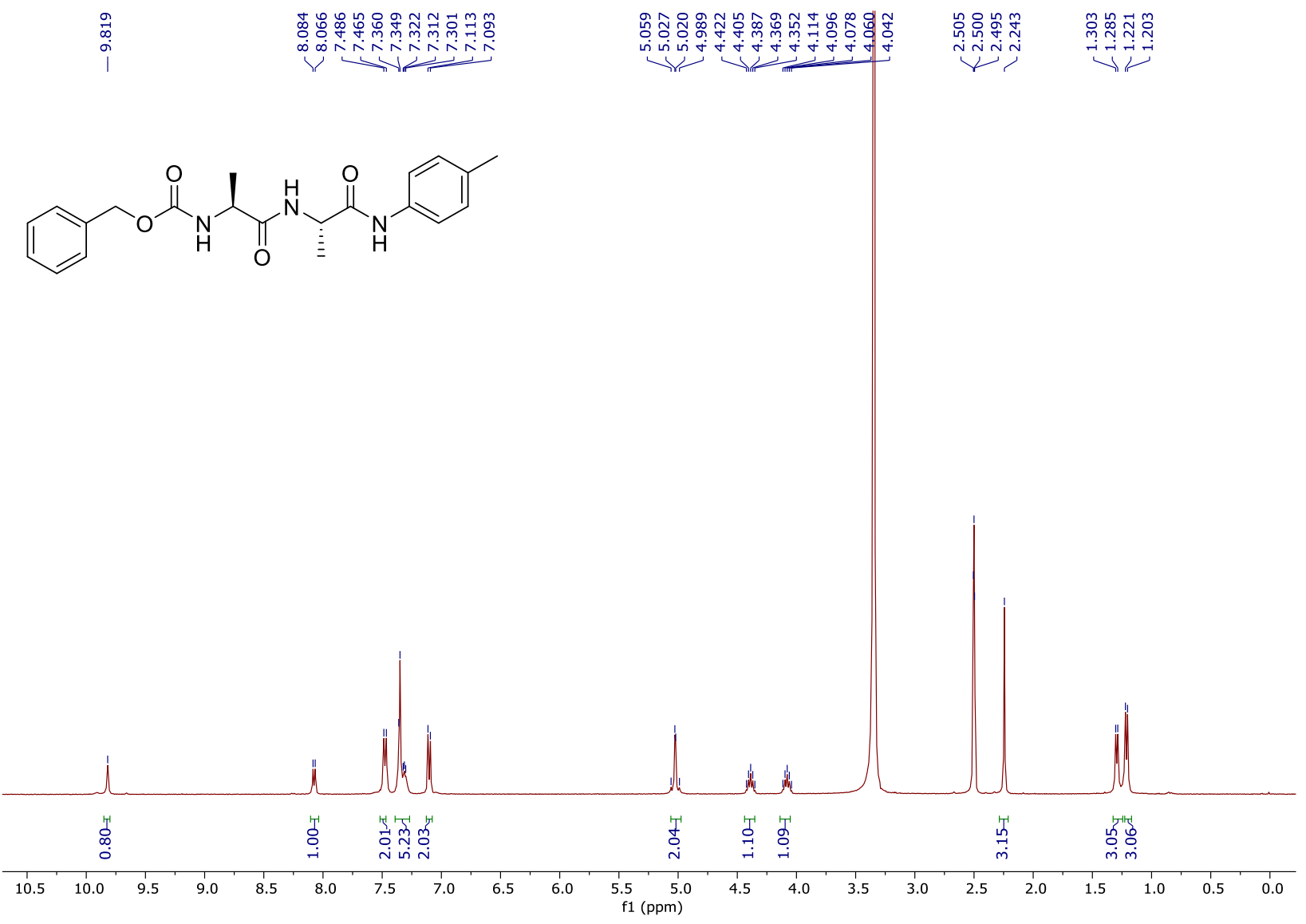


${ }^{13} \mathrm{C}\left\{{ }^{1} \mathrm{H}\right\}$ NMR (101 MHz, DMSO- $\left.d_{6}\right)$ spectra of $\mathbf{6 b c}$

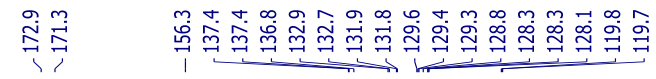

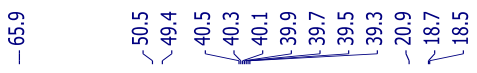

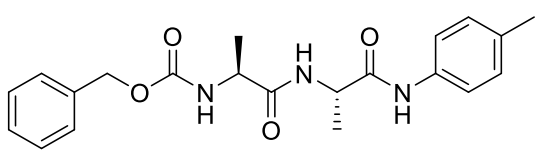
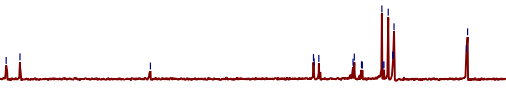

180

$170 \quad 160 \quad 150$

$140 \quad 130$

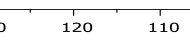

$100 \quad 90$

HRMS of $6 \mathrm{bc}$

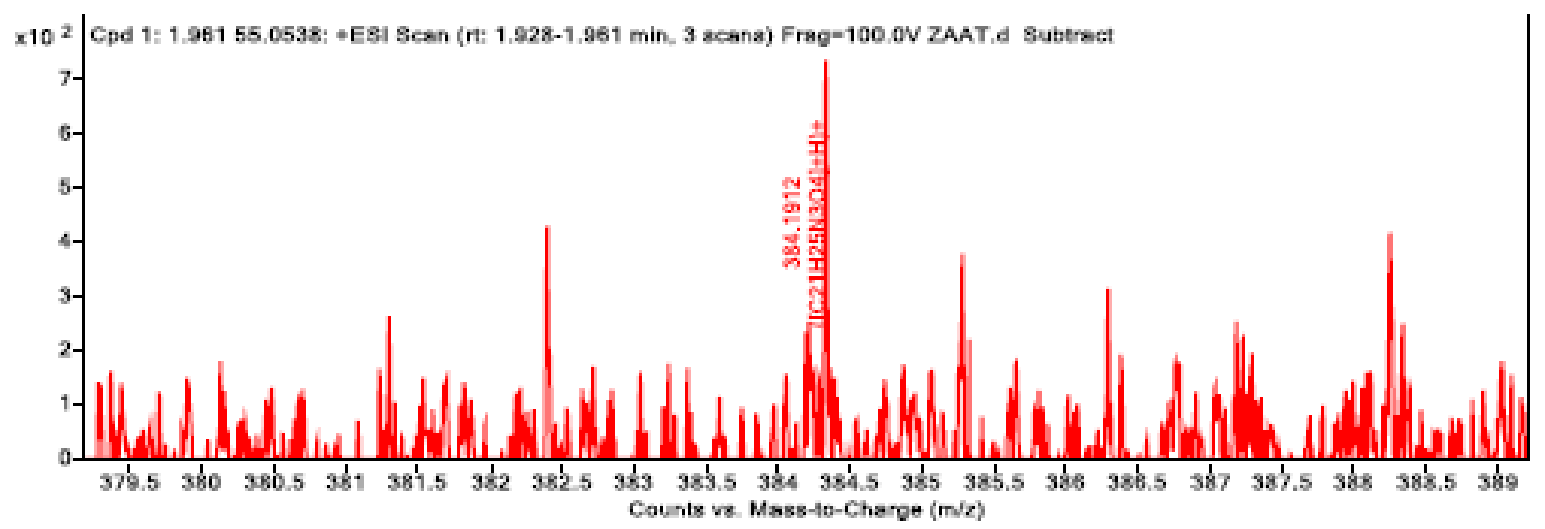


${ }^{1} \mathrm{H}$ NMR (400 MHz, DMSO- $\left.d_{6}\right)$ spectra of $\mathbf{6 c b}$

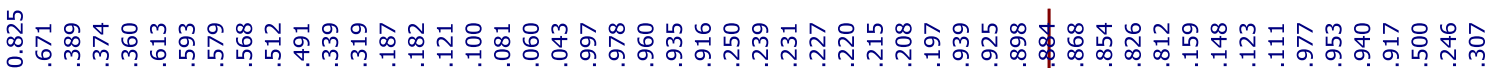

-

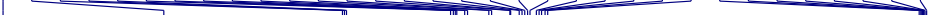<smiles>Cc1ccc(NC(=O)CNC(=O)[C@H](Cc2c[nH]c3ccccc23)NC(=O)OC(C)(C)C)cc1</smiles>

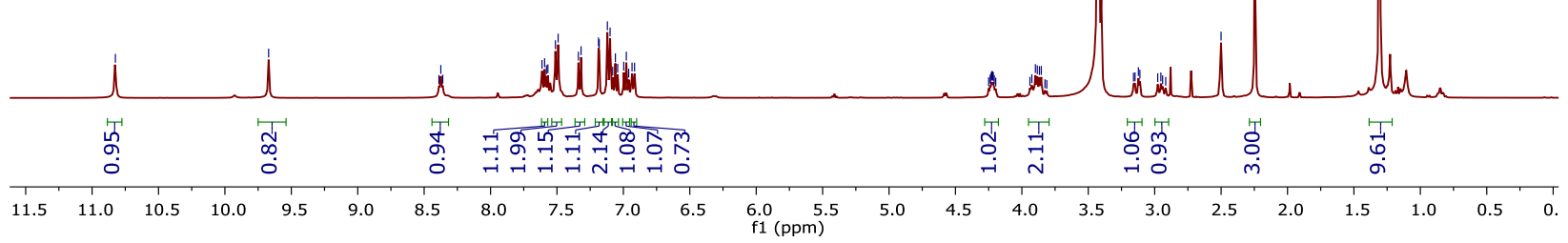

${ }^{13} \mathrm{C}\left\{{ }^{1} \mathrm{H}\right\}$ NMR $\left(101 \mathrm{MHz}, \mathrm{DMSO}-d_{6}\right)$ spectra of $\mathbf{6 c b}$

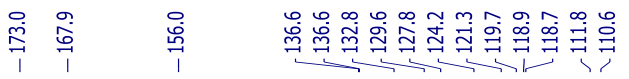

我<smiles>Cc1ccc(NC(=O)CNC(=O)C(Cc2c[nH]c3ccccc23)NC(=O)OC(C)(C)C)cc1</smiles>

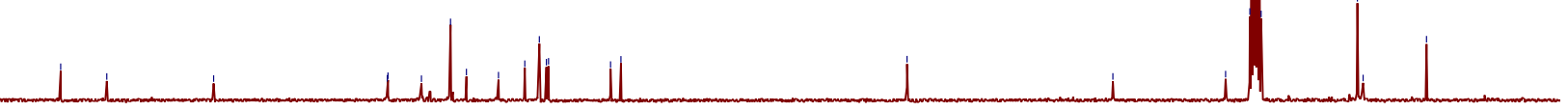

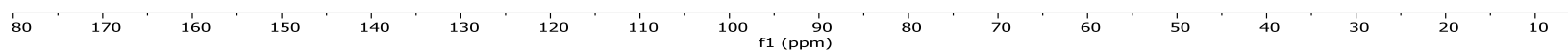




\section{HRMS of $6 \mathrm{cb}$}

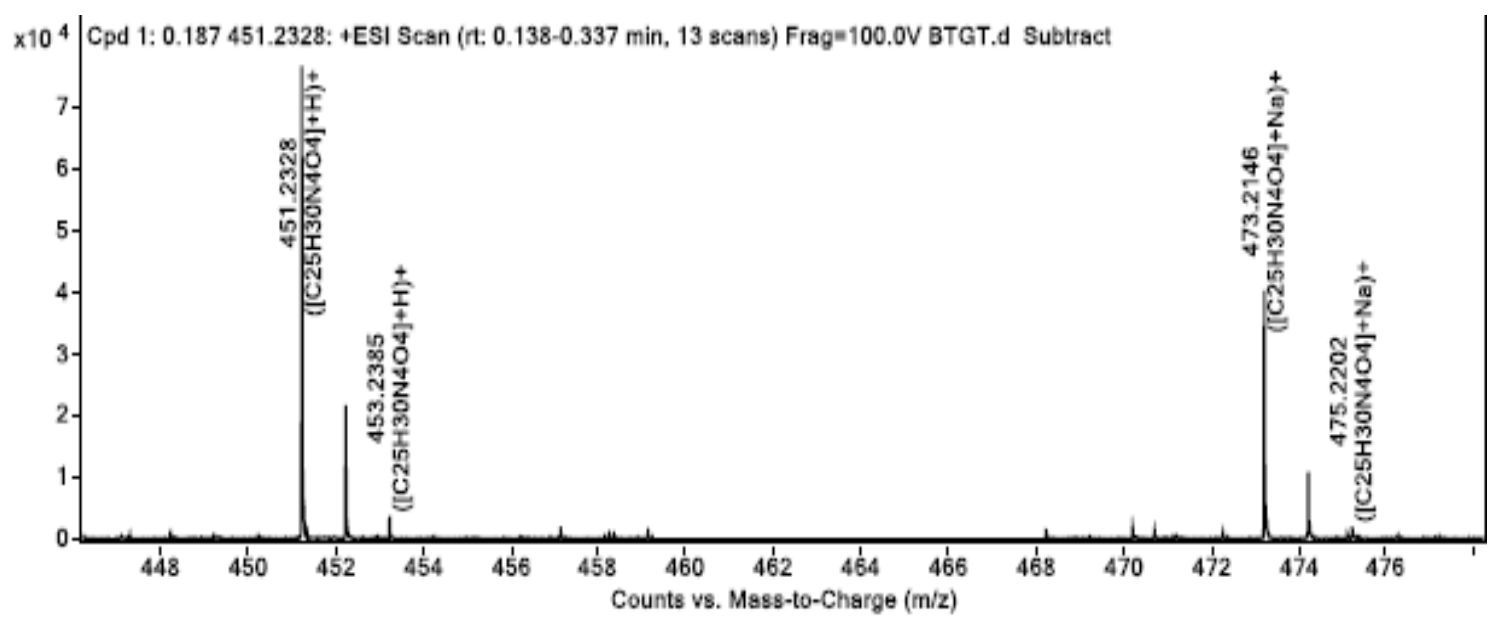

${ }^{1} \mathrm{H}$ NMR (400 MHz, DMSO-d $)$ spectra of $\mathbf{6 d b}$<smiles>Cc1ccc(NC(=O)CNC(=O)[C@H](CCC(=O)OCc2ccccc2)NC(=O)OC(C)(C)C)cc1</smiles>

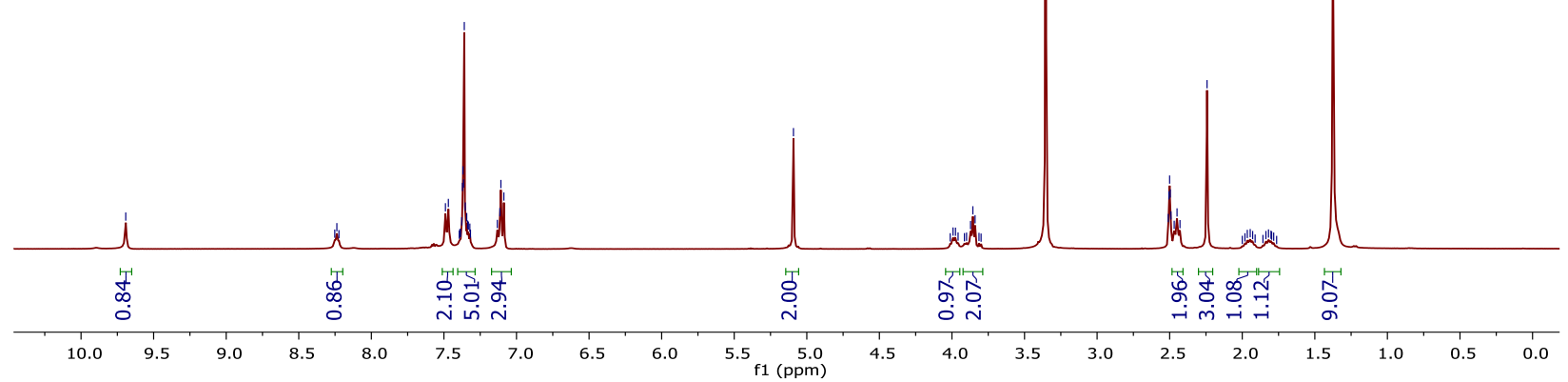


${ }^{13} \mathrm{C}\left\{{ }^{1} \mathrm{H}\right\}$ NMR (101 MHz, DMSO- $\left.d_{6}\right)$ spectra of $\mathbf{6 d b}$

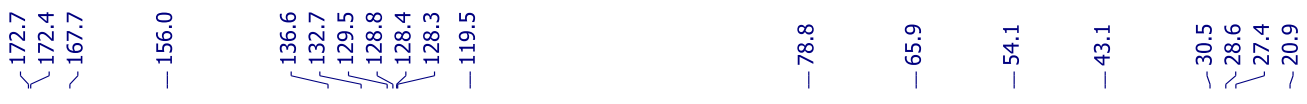<smiles>Cc1ccc(NC(=O)CNC(=O)[C@H](CCC(=O)OCc2ccccc2)NC(=O)OC(C)(C)C)cc1</smiles>
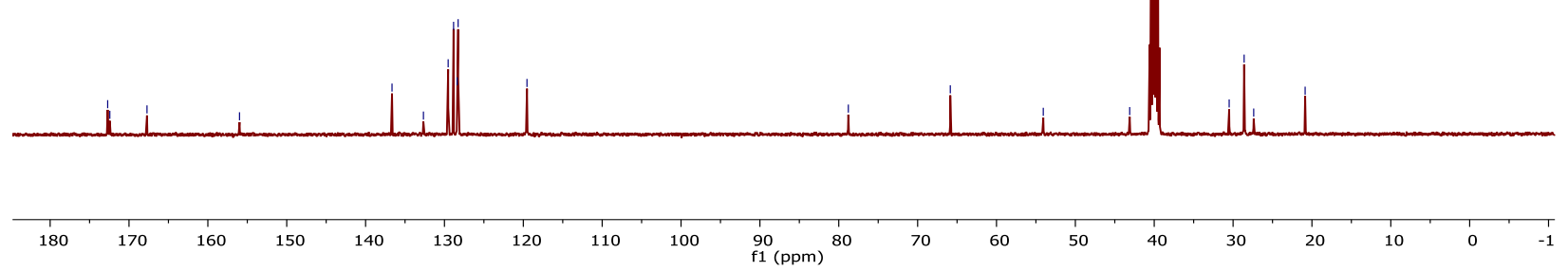

\section{HRMS of 6db}

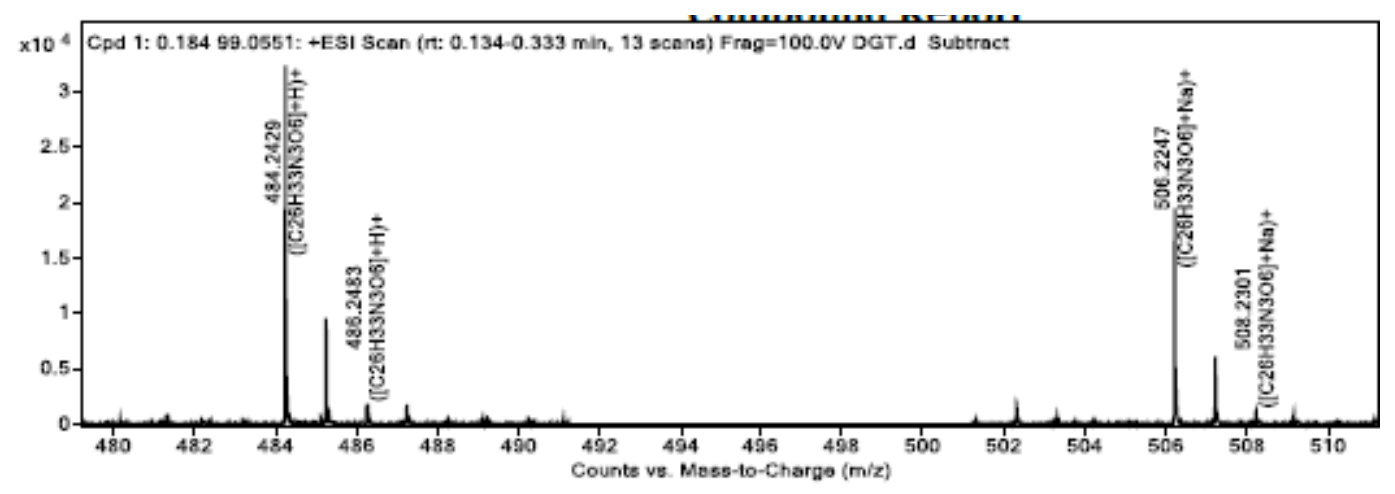


${ }^{1} \mathrm{H}$ NMR (400 MHz, Chloroform- $d$ ) spectra of $6 \mathbf{d c}$

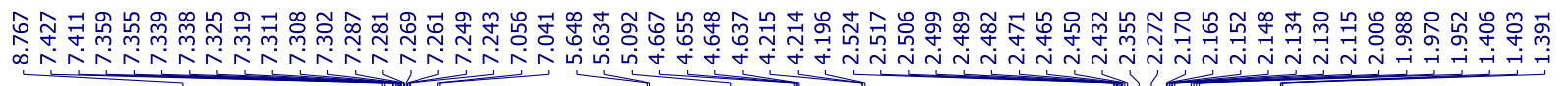<smiles>Cc1ccc(NC(=O)C(C)NC(=O)[C@H](CCC(=O)OCc2ccccc2)NC(=O)OC(C)(C)C)cc1</smiles>

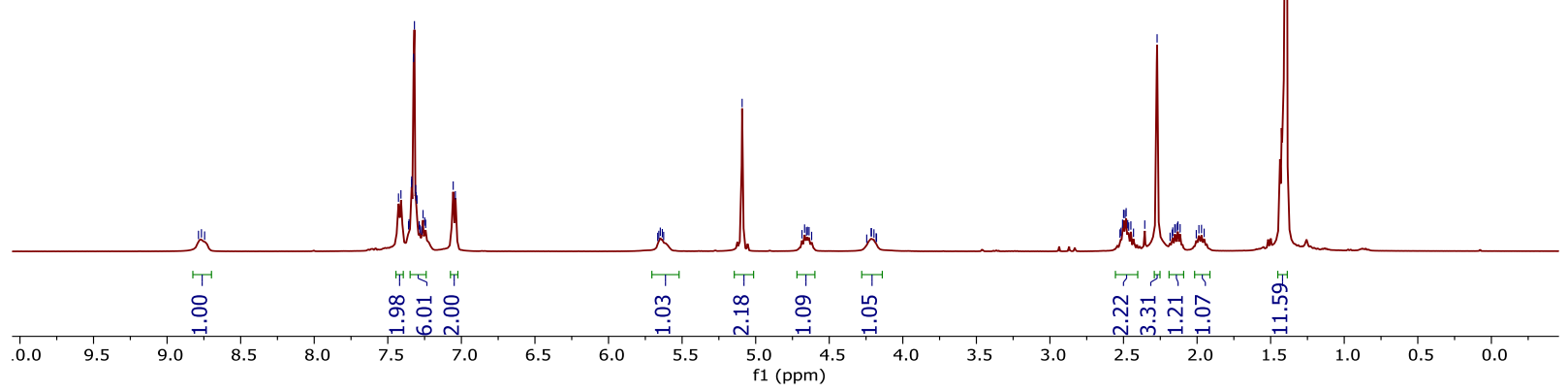

${ }^{13} \mathrm{C}\left\{{ }^{1} \mathrm{H}\right\}$ NMR (101 MHz, Chloroform- $d$ ) spectra of $6 \mathbf{d c}$

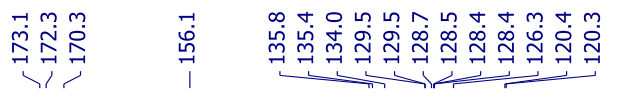

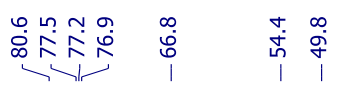

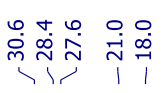<smiles>Cc1ccc(NC(=O)[C@H](C)NC(=O)[C@H](CCC(=O)OCc2ccccc2)NC(=O)OC(C)(C)C)cc1</smiles>
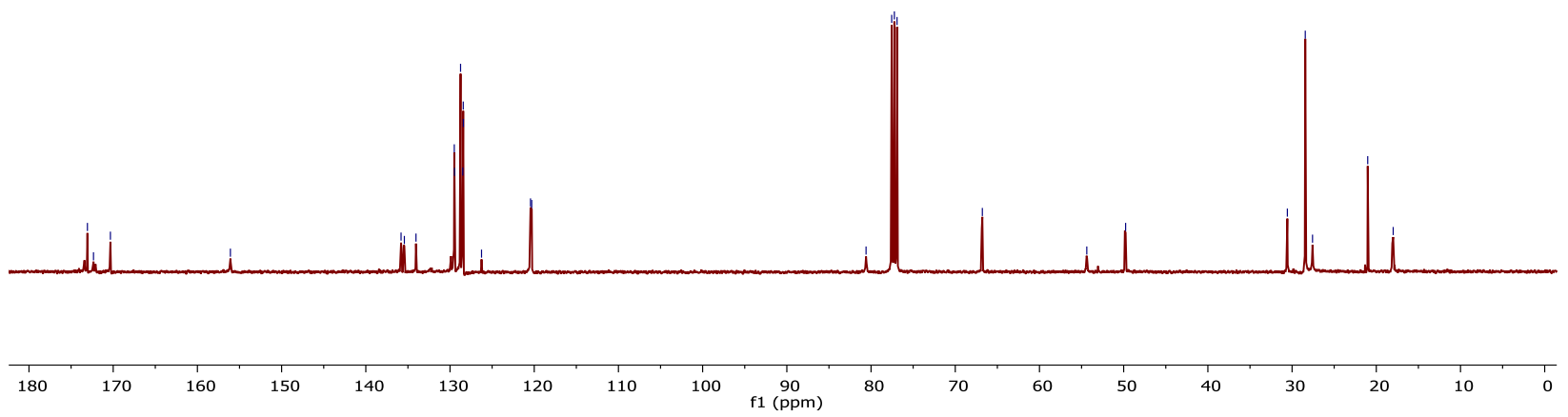

S49 


\section{HRMS of 6dc}

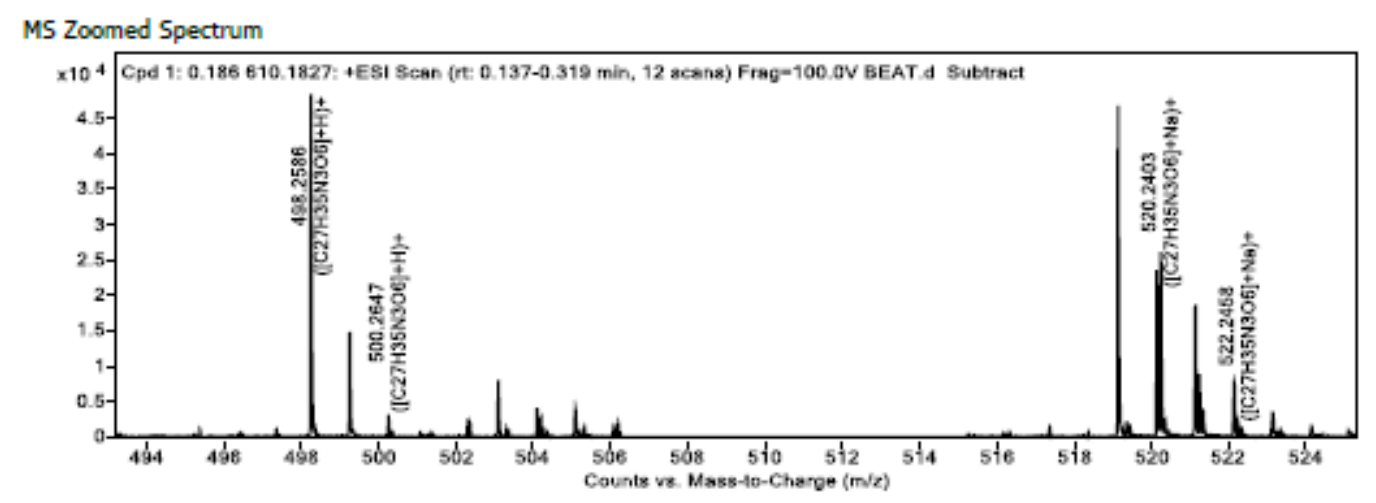

${ }^{1} \mathrm{H}$ NMR (400 MHz, DMSO- $d_{6}$ ) spectra of $\mathbf{6 e b}$

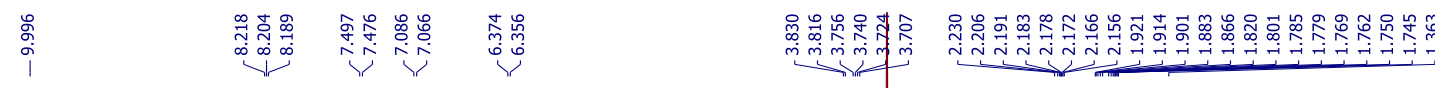<smiles>Cc1ccc(NC(=O)CNC(=O)C(CCC(=O)O)NC(=O)OC(C)(C)C)cc1</smiles>

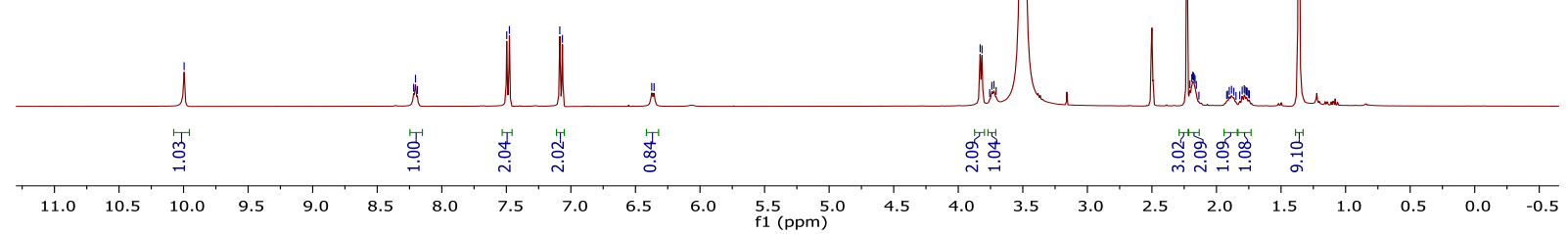




\section{HRMS of $\mathbf{6 e b}$}

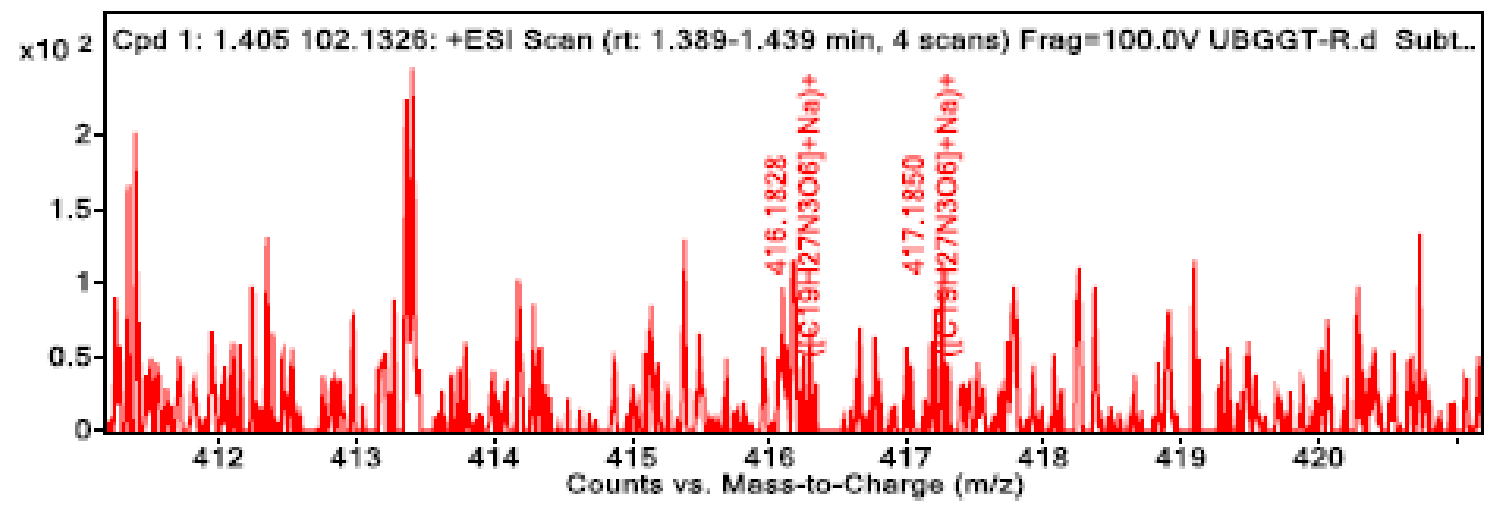

${ }^{1} \mathrm{H}$ NMR (400 MHz, DMSO- $d_{6}$ ) spectra of $\mathbf{6 f h}$

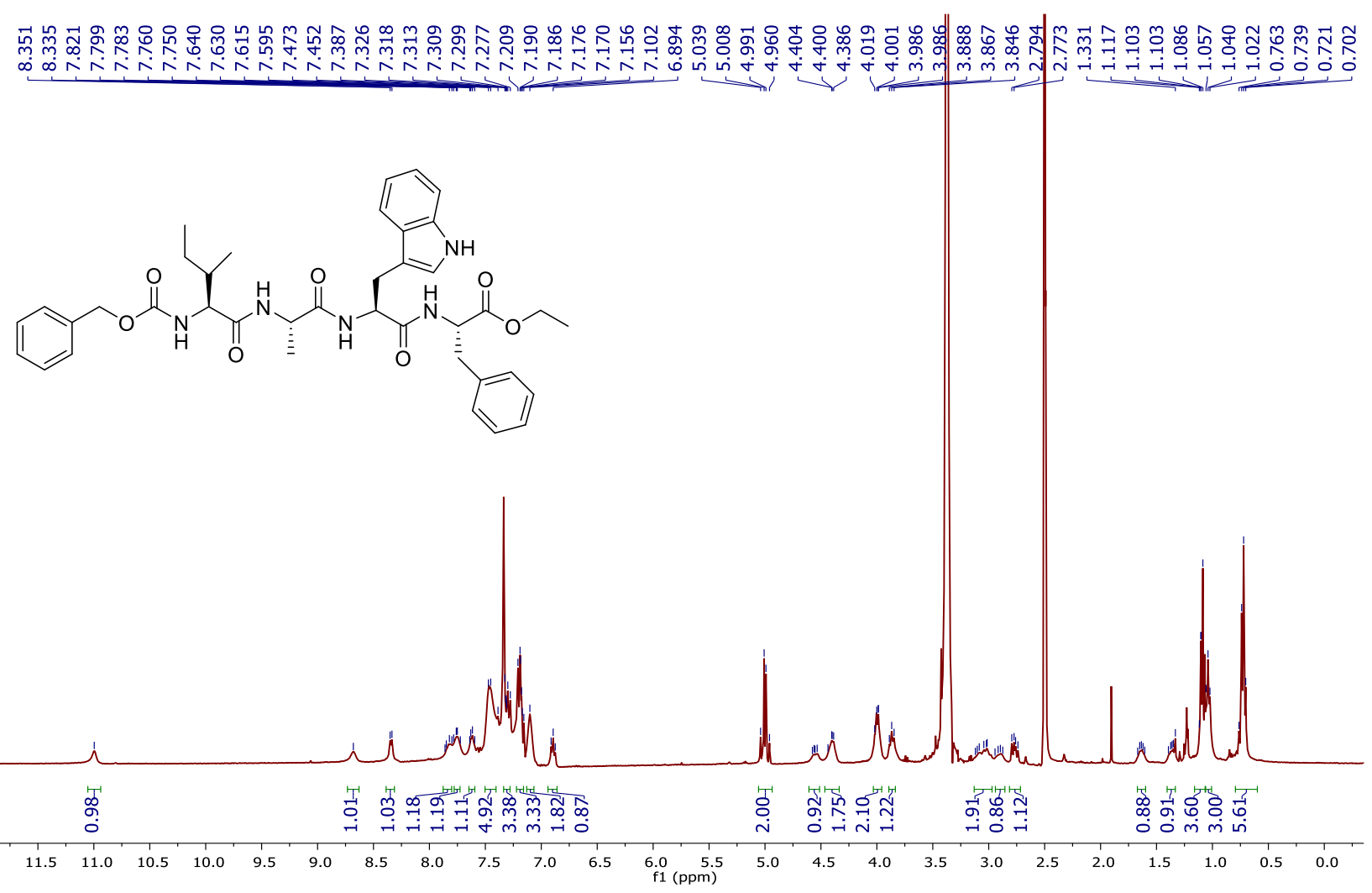


${ }^{13} \mathrm{C}\left\{{ }^{1} \mathrm{H}\right\}$ NMR (101 MHz, DMSO- $\left.d_{6}\right)$ spectra of $\mathbf{6 f h}$

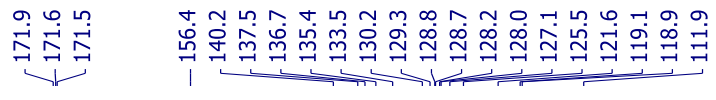

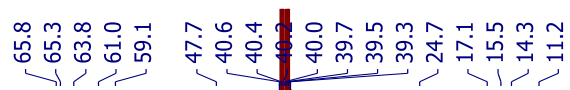

> $>$
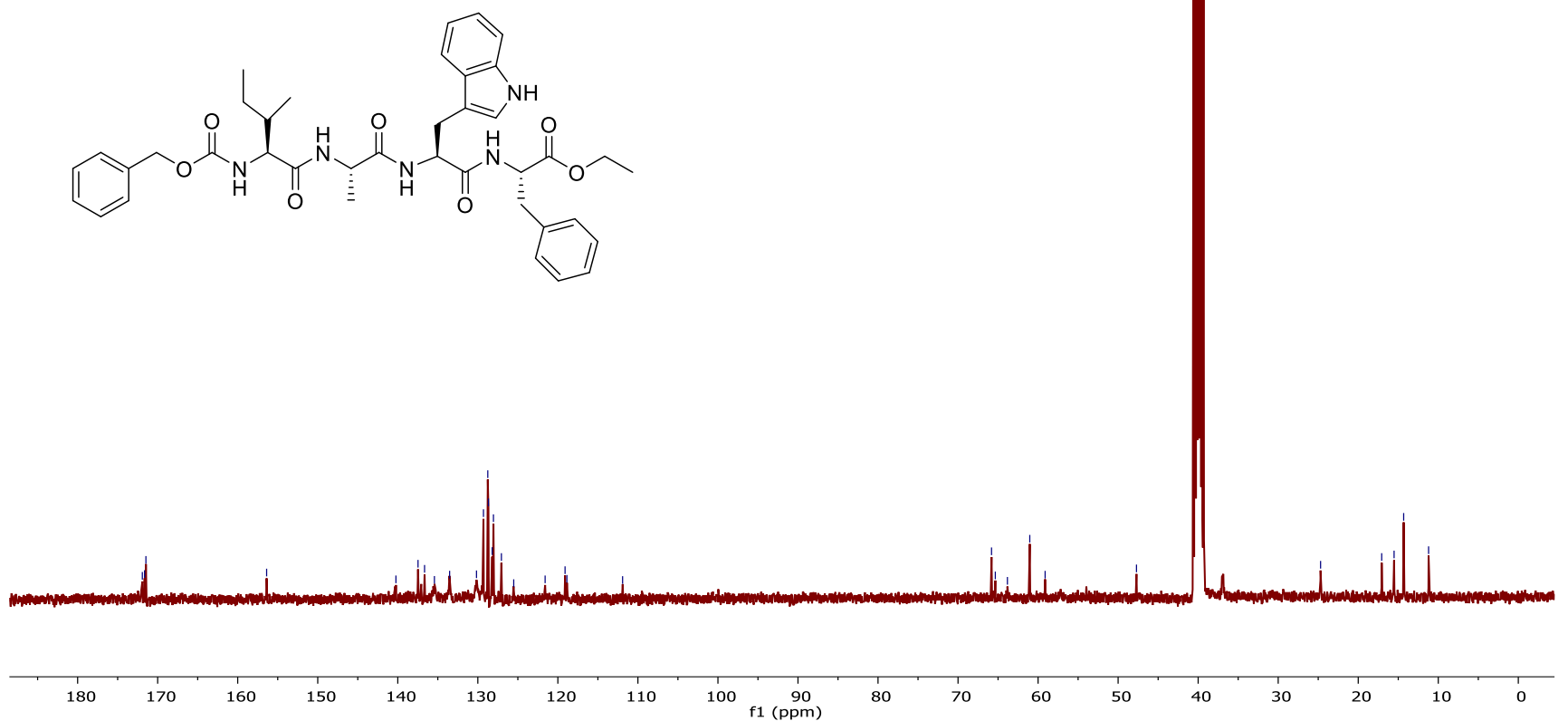

HRMS of $6 \mathrm{fh}$

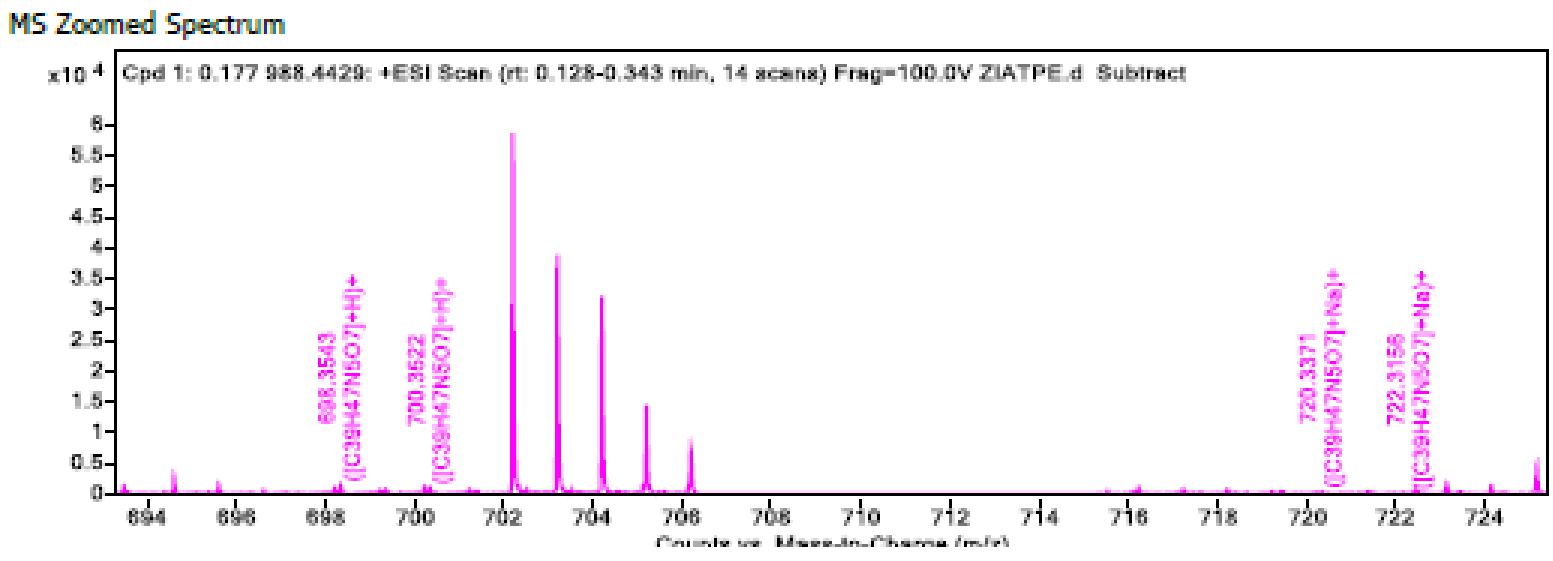




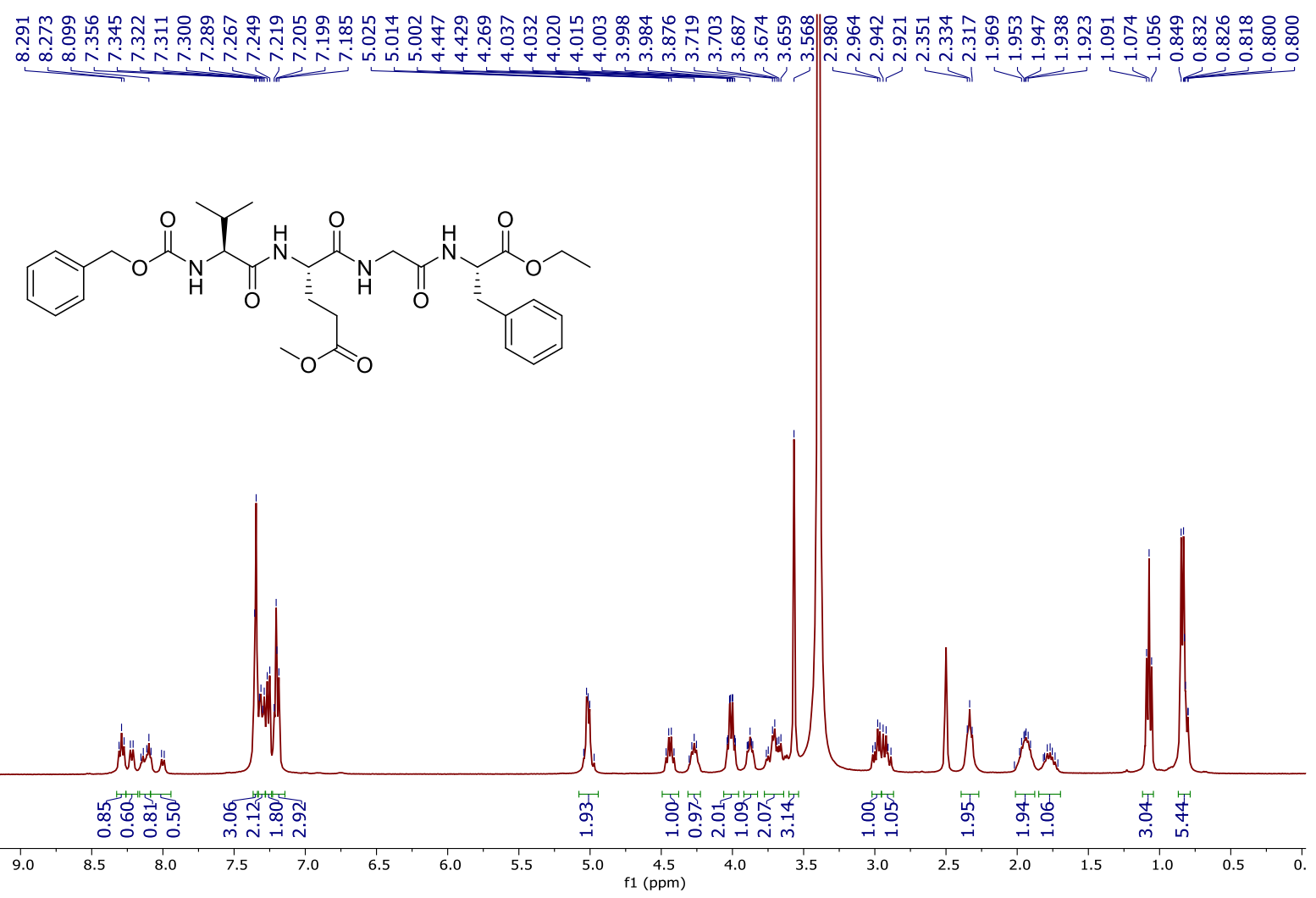

${ }^{13} \mathrm{C}\left\{{ }^{1} \mathrm{H}\right\}$ NMR (101 MHz, DMSO- $\left.d_{6}\right)$ spectra of $\mathbf{6 g i}$

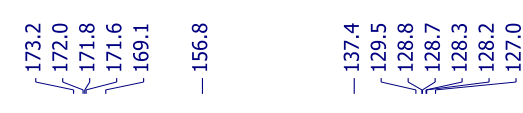

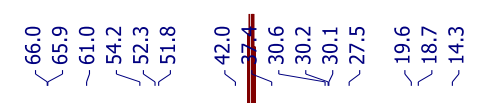<smiles>CCOC(=O)[C@H](Cc1ccccc1)NC(=O)CNC(=O)[C@H](CCC(=O)OC)NC(=O)C(NC(=O)OCc1ccccc1)C(C)C</smiles>

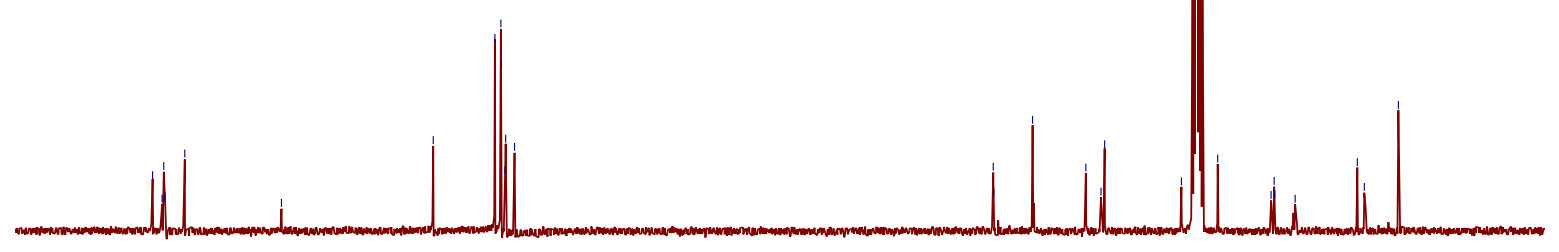




\section{HRMS of 6gi}

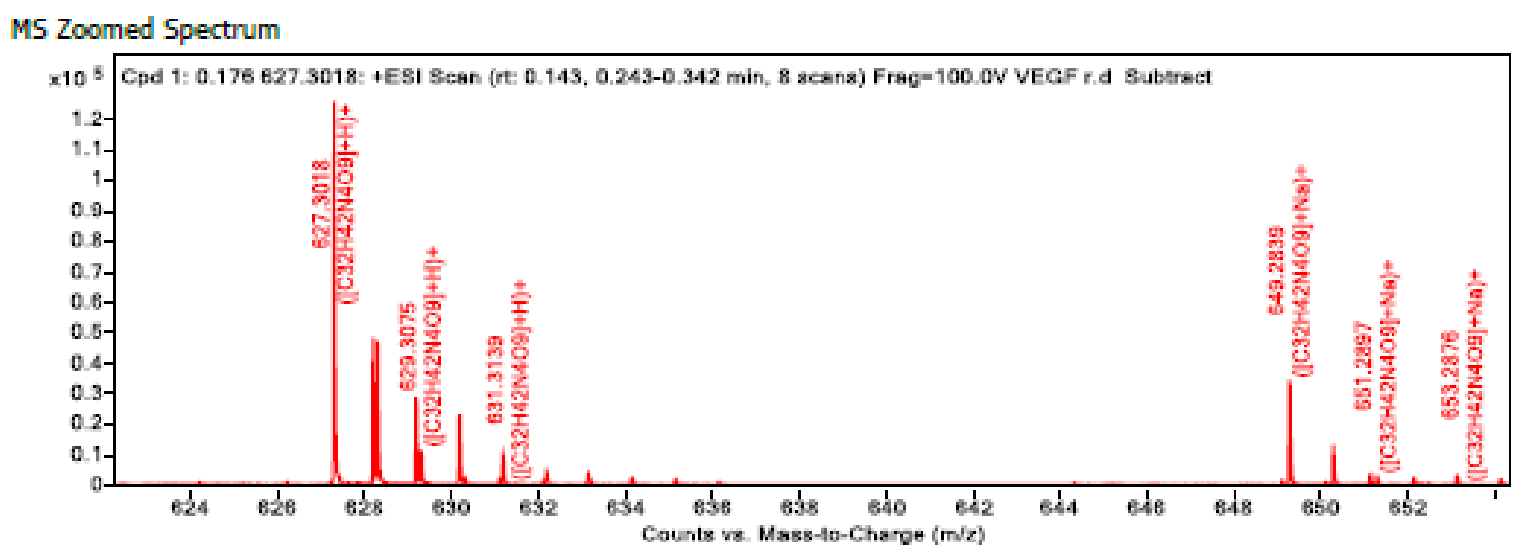

${ }^{1} \mathrm{H}$ NMR (400 MHz, DMSO- $\left.d_{6}\right)$ spectra of $6 \mathbf{h a}$

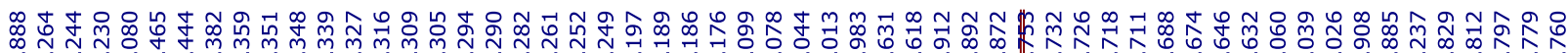
向<smiles>CCC(C)C(NC(=O)OCc1ccccc1)C(=O)NCC(=O)NCC(=O)N[C@@H](Cc1ccccc1)C(=O)Nc1ccc(C)cc1</smiles>

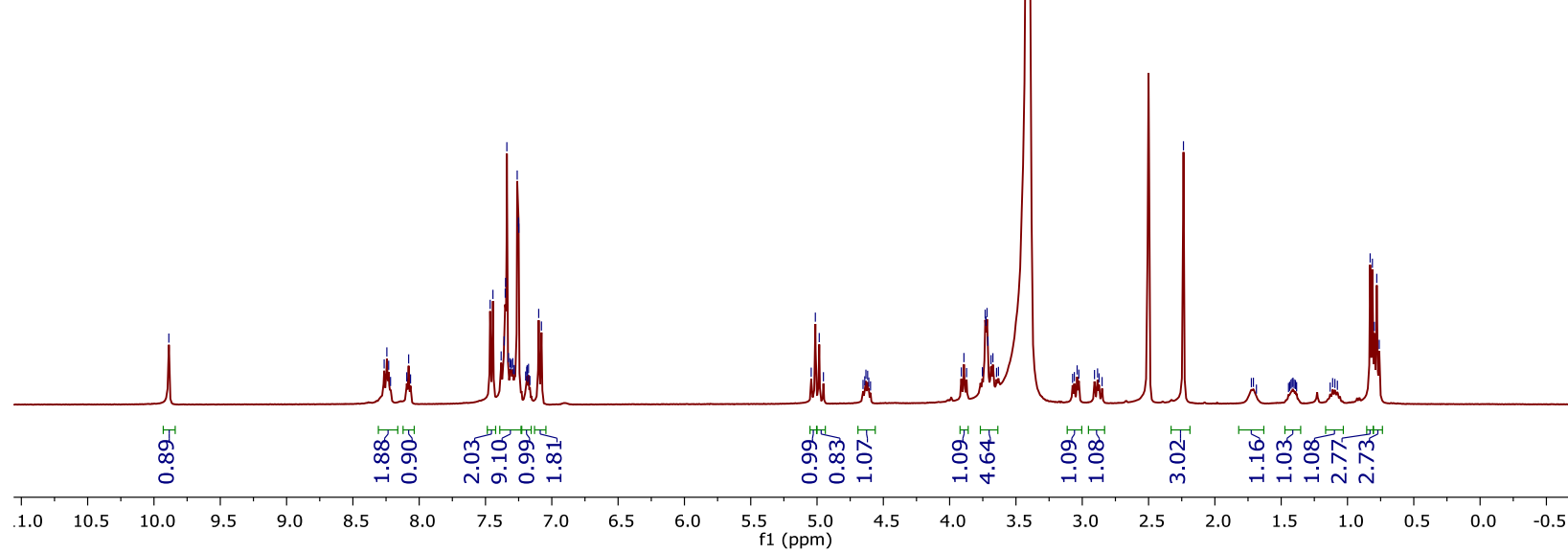


${ }^{13} \mathrm{C}\left\{{ }^{1} \mathrm{H}\right\}$ NMR (101 MHz, DMSO- $d_{6}$ ) spectra of 6ha

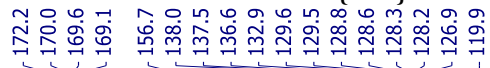

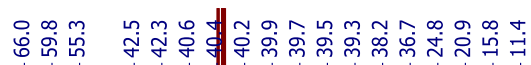

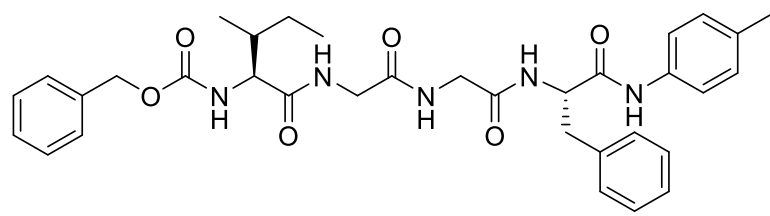
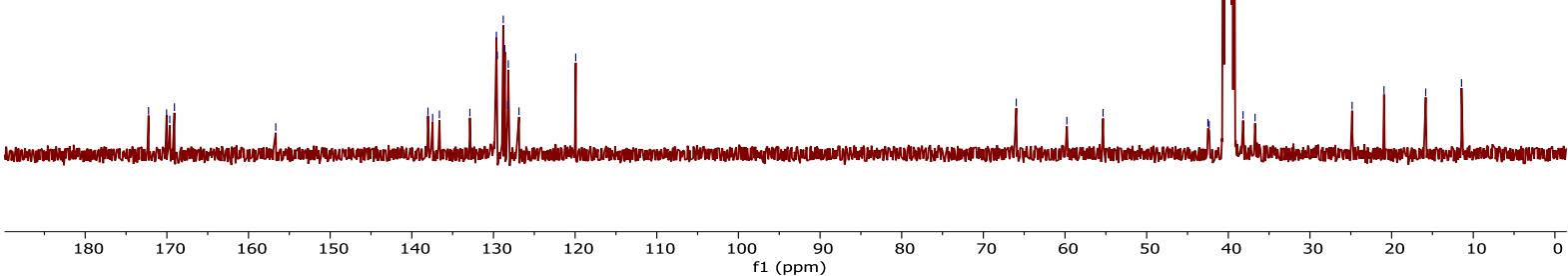

\section{HRMS of $6 \mathrm{ha}$}

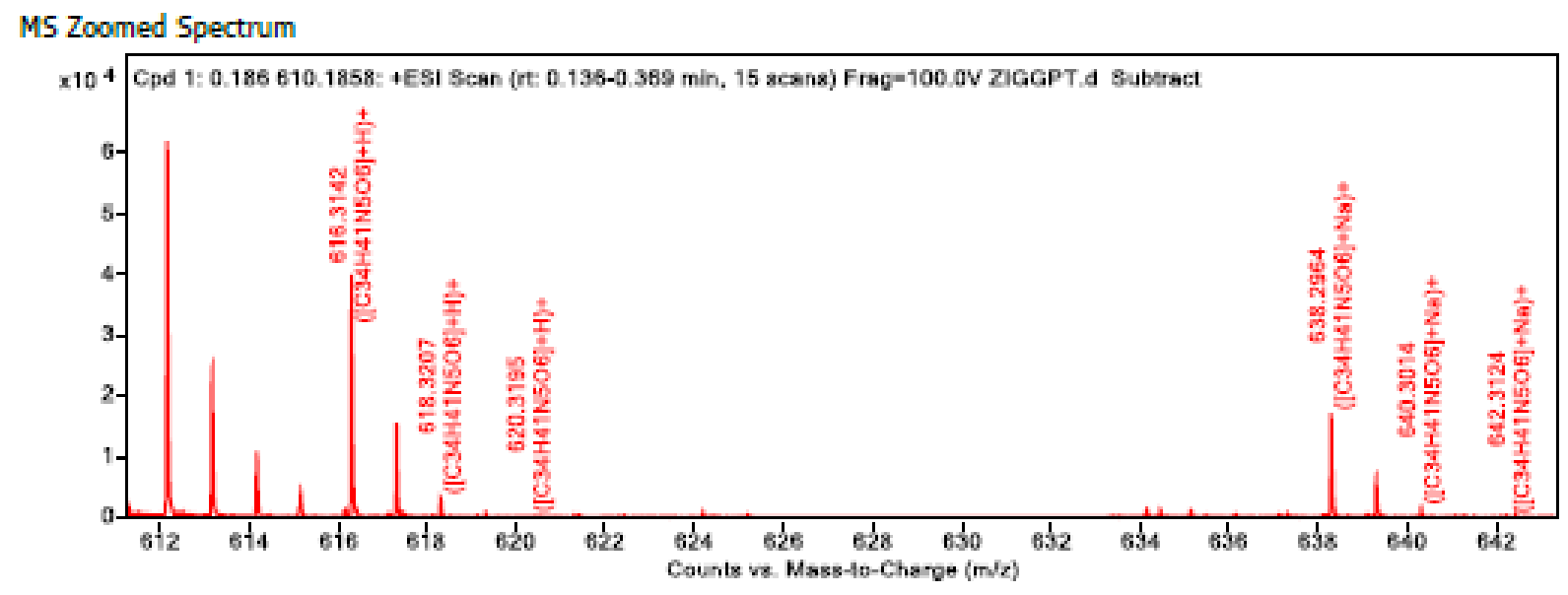


${ }^{1} \mathrm{H}$ NMR (400 MHz, DMSO-d ${ }_{6}$ ) spectra of $\mathbf{6 h \mathbf { h }}$

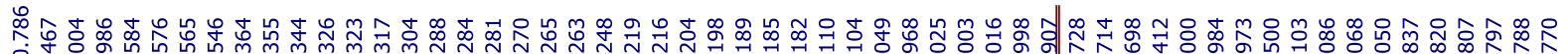
定<smiles>CCOC(=O)C(Cc1ccccc1)NC(=O)C(Cc1c[nH]c2ccccc12)NC(=O)CNC(=O)CNC(=O)C(NC(=O)OCc1ccccc1)C(C)CC</smiles>

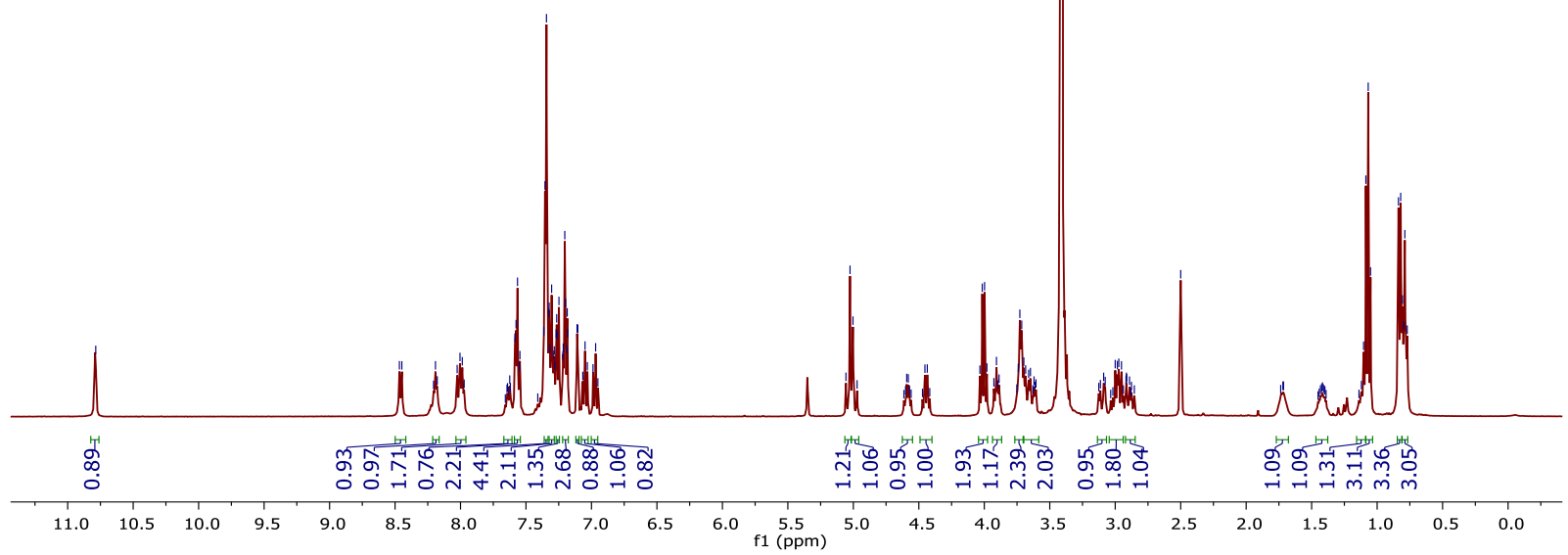

${ }^{13} \mathrm{C}\left\{{ }^{1} \mathrm{H}\right\}$ NMR (101 MHz, DMSO- $d_{6}$ ) spectra of $\mathbf{6 h h}$

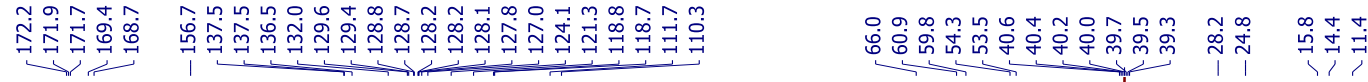<smiles>CCOC(=O)C(Cc1ccccc1)NC(=O)[C@H](Cc1c[nH]c2ccccc12)NC(=O)CNC(=O)CNC(=O)C(NC(=O)OCc1ccccc1)C(C)CC</smiles>
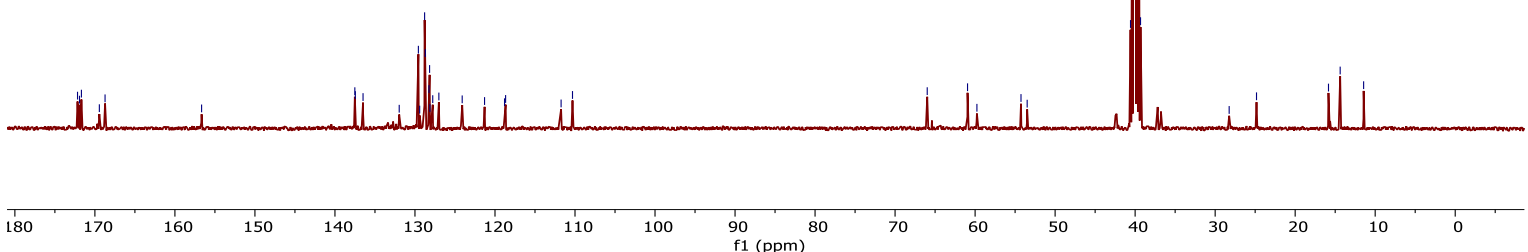


\section{HRMS of $6 \mathrm{hh}$}

\section{MS Zoomed Spectrum}

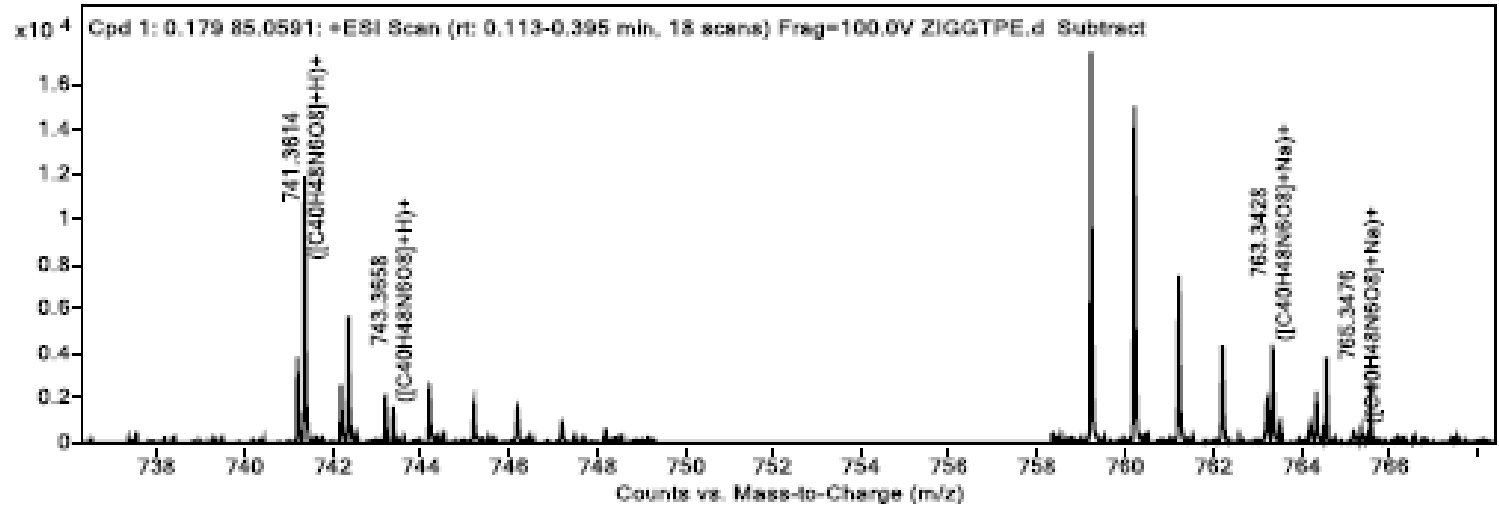

${ }^{1} \mathrm{H}$ NMR (400 MHz, Chloroform- $d$ ) spectra of $6 \mathbf{i}^{\prime} \mathbf{h}$

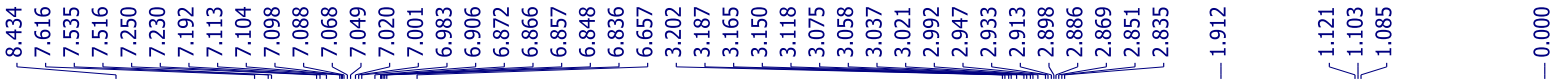<smiles>CCOC(=O)C(Cc1ccccc1)NC(=O)C(Cc1c[nH]c2ccccc12)NC(=O)CNC(=O)CN</smiles>

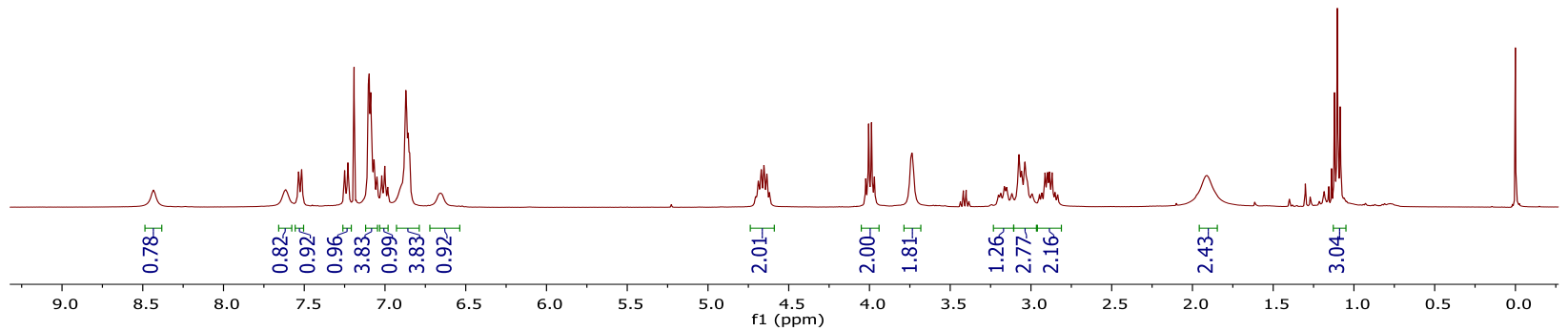


${ }^{13} \mathrm{C}\left\{{ }^{1} \mathrm{H}\right\}$ NMR (101 MHz, Chloroform- $d$ ) spectra of $\mathbf{6} \mathbf{i}^{\prime} \mathbf{h}$

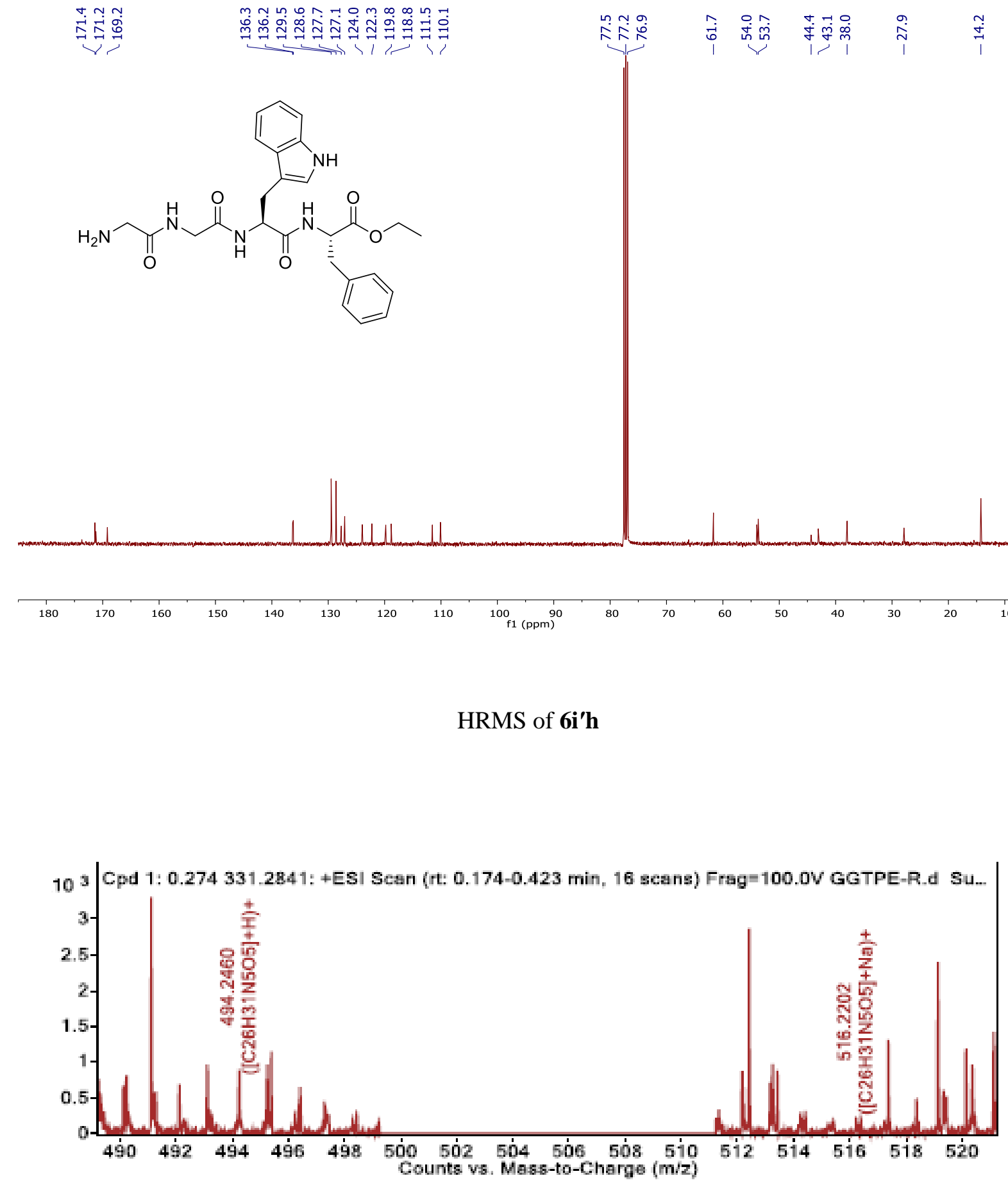


4. Comparison of ${ }^{1} \mathrm{H}$ NMR of Cbz-L-Ala-L-Ala- $p$-tol (6bc) with Cbz-L-Ala-DL-Ala- $p$-tol

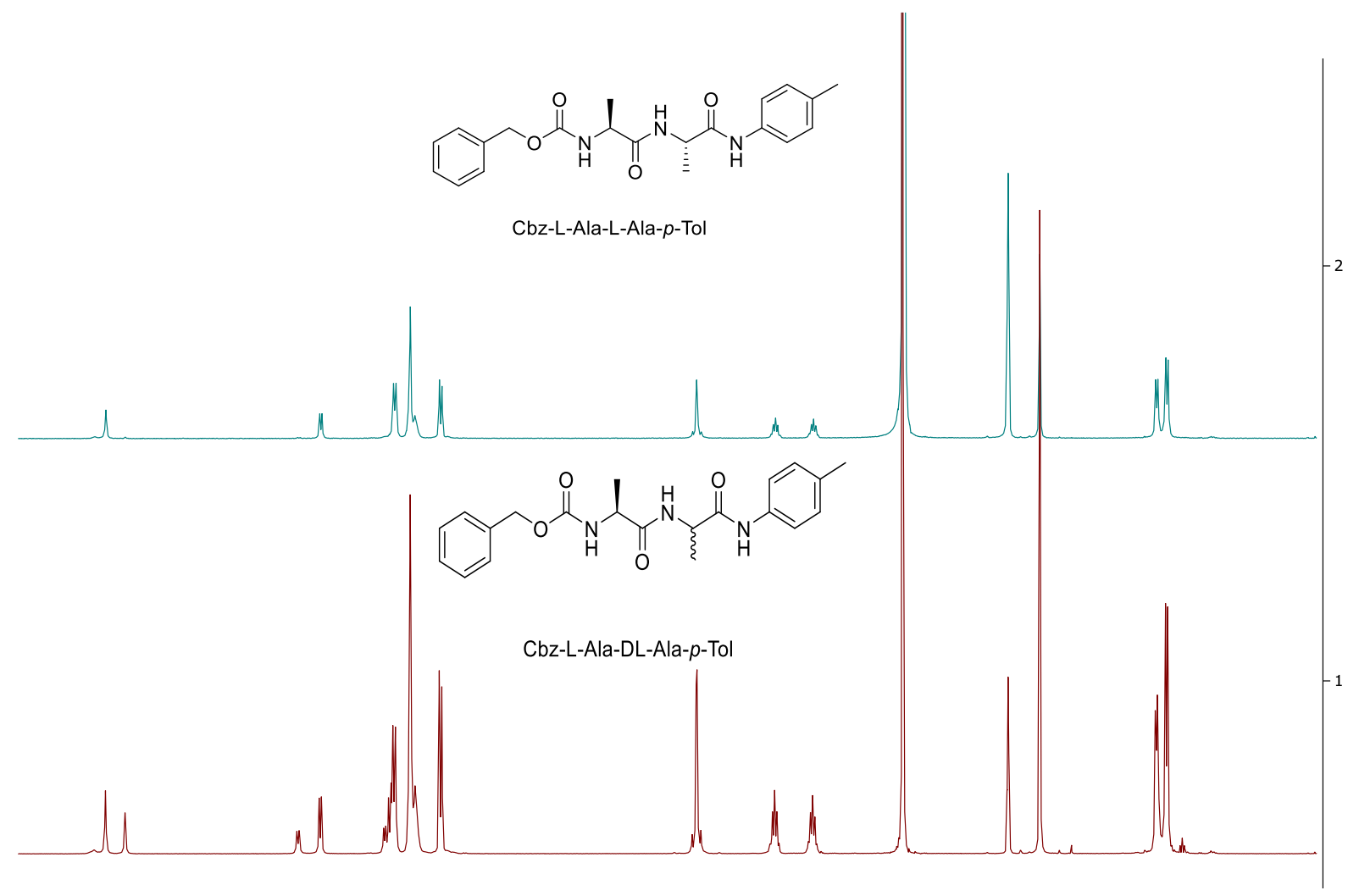

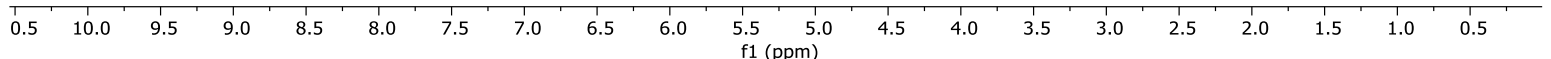




\section{Computational data: coordinates, imaginary frequency and energy data.}

Computational data of Figure 1:

Compound ID : 4a

Calculation : OPT + FREQ, Basis Set : TZVP, Functional : BP86

Imaginary Frequency $=0$

Dipole Moment $=5.04$ debye

$\mathrm{EE}=-0.15210221$ Hartree

$\begin{array}{lrrr}\mathrm{C} & 8.6545000000 & 1.3988000000 & -1.5936000000 \\ \mathrm{C} & 9.5419000000 & 0.5440000000 & -0.9481000000 \\ \mathrm{C} & 9.1356000000 & -0.1529000000 & 0.1851000000 \\ \mathrm{C} & 7.8437000000 & 0.0040000000 & 0.6747000000 \\ \mathrm{C} & 6.9523000000 & 0.8615000000 & 0.0292000000 \\ \mathrm{C} & 7.3613000000 & 1.5580000000 & -1.1083000000 \\ \mathrm{C} & 5.5624000000 & 1.0439000000 & 0.5643000000 \\ \mathrm{O} & 4.7727000000 & 0.0005000000 & -0.0182000000 \\ \mathrm{C} & 3.4558000000 & -0.0564000000 & 0.3299000000 \\ \mathrm{~N} & 2.8691000000 & -1.2428000000 & -0.1904000000 \\ \mathrm{O} & 2.9237000000 & 0.7721000000 & 1.0522000000 \\ \mathrm{C} & 1.4023000000 & -1.2405000000 & -0.4034000000 \\ \mathrm{C} & 0.9005000000 & -2.6711000000 & -0.4314000000 \\ \mathrm{O} & -0.2142000000 & -3.1195000000 & 0.2145000000 \\ \mathrm{O} & 1.4055000000 & -3.6153000000 & -1.0032000000 \\ \mathrm{C} & -1.0865000000 & -2.2149000000 & 0.8822000000 \\ \mathrm{C} & -2.4831000000 & -2.3516000000 & 0.3266000000 \\ \mathrm{C} & -3.2192000000 & -1.2689000000 & -0.1666000000 \\ \mathrm{C} & -4.5232000000 & -1.5031000000 & -0.5991000000 \\ \mathrm{C} & -5.0916000000 & -2.7702000000 & -0.5680000000 \\ \mathrm{C} & -4.3479000000 & -3.8429000000 & -0.0900000000 \\ \mathrm{C} & -3.0525000000 & -3.6300000000 & 0.3608000000 \\ \mathrm{P} & -2.5708000000 & 0.4534000000 & -0.3292000000 \\ \mathrm{C} & -3.8304000000 & 1.4001000000 & -1.2417000000 \\ \mathrm{C} & -2.6255000000 & 1.0990000000 & 1.3696000000 \\ \mathrm{C} & -4.9654000000 & 1.9358000000 & -0.6395000000 \\ \mathrm{C} & -5.8815000000 & 2.6774000000 & -1.3780000000 \\ \mathrm{C} & -5.6662000000 & 2.8915000000 & -2.7359000000 \\ \mathrm{C} & -4.5336000000 & 2.3646000000 & -3.3469000000 \\ \mathrm{C} & -3.6219000000 & 1.6237000000 & -2.5999000000 \\ \mathrm{C} & -1.6372000000 & 1.9904000000 & 1.7763000000 \\ \mathrm{C} & -1.6396000000 & 2.5118000000 & 3.0673000000 \\ \mathrm{C} & -2.6363000000 & 2.1418000000 & 3.9636000000 \\ \mathrm{C} & -3.6253000000 & 1.2463000000 & 3.5678000000 \\ \mathrm{C} & -3.6146000000 & 0.7293000000 & 2.2767000000 \\ \mathrm{O} & -1.2290000000 & 0.5090000000 & -0.9603000000 \\ & & & \end{array}$




$\begin{array}{cccc}\mathrm{H} & 8.9733000000 & 1.9465000000 & -2.4865000000 \\ \mathrm{H} & 10.5595000000 & 0.4191000000 & -1.3323000000 \\ \mathrm{H} & 9.8330000000 & -0.8274000000 & 0.6925000000 \\ \mathrm{H} & 7.5234000000 & -0.5473000000 & 1.5661000000 \\ \mathrm{H} & 6.6626000000 & 2.2300000000 & -1.6196000000 \\ \mathrm{H} & 5.5372000000 & 0.9601000000 & 1.6677000000 \\ \mathrm{H} & 5.1421000000 & 2.0293000000 & 0.2857000000 \\ \mathrm{H} & 3.3677000000 & -1.6733000000 & -0.9412000000 \\ \mathrm{H} & 1.1066000000 & -0.7421000000 & -1.3540000000 \\ \mathrm{H} & 0.9038000000 & -0.6581000000 & 0.4074000000 \\ \mathrm{H} & -0.7238000000 & -1.1637000000 & 0.8537000000 \\ \mathrm{H} & -1.0707000000 & -2.5490000000 & 1.9366000000 \\ \mathrm{H} & -5.1201000000 & -0.6624000000 & -0.9813000000 \\ \mathrm{H} & -4.7801000000 & -4.8488000000 & -0.0670000000 \\ \mathrm{H} & -2.4646000000 & -4.4745000000 & 0.7413000000 \\ \mathrm{H} & -5.1330000000 & 1.7776000000 & 0.4349000000 \\ \mathrm{H} & -6.7690000000 & 3.0943000000 & -0.8908000000 \\ \mathrm{H} & -6.3850000000 & 3.4753000000 & -3.3202000000 \\ \mathrm{H} & -4.3571000000 & 2.5340000000 & -4.4143000000 \\ \mathrm{H} & -2.7228000000 & 1.2186000000 & -3.0836000000 \\ \mathrm{H} & -0.8413000000 & 2.2757000000 & 1.0747000000 \\ \mathrm{H} & -0.8539000000 & 3.2094000000 & 3.3765000000 \\ \mathrm{H} & -2.6412000000 & 2.5503000000 & 4.9796000000 \\ \mathrm{H} & -4.4083000000 & 0.9471000000 & 4.2722000000 \\ \mathrm{H} & -4.3861000000 & 0.0098000000 & 1.9710000000\end{array}$

Compound ID : 5a

Calculation : OPT + FREQ, Basis Set : TZVP, Functional : BP86

Imaginary Frequency $=0$

Dipole Moment $=2.29$ debye

$\mathrm{EE}=-0.0026998038$ Hartree

$\begin{array}{lrrr}\mathrm{N} & -1.3116000000 & -1.4213000000 & -0.9701000000 \\ \mathrm{C} & -1.1740000000 & -0.2262000000 & -0.1000000000 \\ \mathrm{C} & 0.2679000000 & 0.2856000000 & -0.2547000000 \\ \mathrm{~N} & 1.2378000000 & -0.3546000000 & 0.5753000000 \\ \mathrm{O} & 0.5819000000 & 1.2083000000 & -0.9876000000 \\ \mathrm{C} & 2.6589000000 & -0.2169000000 & 0.3500000000 \\ \mathrm{C} & 3.2604000000 & 1.0460000000 & 0.3411000000 \\ \mathrm{C} & 4.6384000000 & 1.1505000000 & 0.1976000000 \\ \mathrm{C} & 5.4302000000 & 0.0086000000 & 0.0722000000 \\ \mathrm{C} & 4.8250000000 & -1.2492000000 & 0.0866000000 \\ \mathrm{C} & 3.4489000000 & -1.3670000000 & 0.2229000000\end{array}$




$\begin{array}{lrrr}\mathrm{C} & 6.9045000000 & 0.1289000000 & -0.0629000000 \\ \mathrm{C} & -2.1887000000 & 0.8876000000 & -0.3977000000 \\ \mathrm{C} & -3.5836000000 & 0.4538000000 & -0.0804000000 \\ \mathrm{C} & -4.0000000000 & 0.3561000000 & 1.2491000000 \\ \mathrm{C} & -5.2992000000 & -0.0365000000 & 1.5474000000 \\ \mathrm{C} & -6.1921000000 & -0.3317000000 & 0.5219000000 \\ \mathrm{C} & -5.7832000000 & -0.2318000000 & -0.8032000000 \\ \mathrm{C} & -4.4833000000 & 0.1597000000 & -1.1057000000 \\ \mathrm{H} & -2.2200000000 & -1.8151000000 & -0.8370000000 \\ \mathrm{H} & -1.2096000000 & -1.1552000000 & -1.9279000000 \\ \mathrm{H} & -1.3411000000 & -0.5744000000 & 0.9525000000 \\ \mathrm{H} & 0.9849000000 & -1.2874000000 & 0.8245000000 \\ \mathrm{H} & 2.6556000000 & 1.9562000000 & 0.4316000000 \\ \mathrm{H} & 5.1071000000 & 2.1412000000 & 0.1827000000 \\ \mathrm{H} & 5.4405000000 & -2.1503000000 & -0.0142000000 \\ \mathrm{H} & 2.9958000000 & -2.3660000000 & 0.2312000000 \\ \mathrm{H} & 7.1934000000 & 1.0640000000 & -0.5607000000 \\ \mathrm{H} & 7.3817000000 & 0.1201000000 & 0.9267000000 \\ \mathrm{H} & 7.3303000000 & -0.7009000000 & -0.6424000000 \\ \mathrm{H} & -2.1110000000 & 1.2144000000 & -1.4556000000 \\ \mathrm{H} & -1.9380000000 & 1.7826000000 & 0.2067000000 \\ \mathrm{H} & -3.2987000000 & 0.5946000000 & 2.0572000000 \\ \mathrm{H} & -5.6205000000 & -0.1108000000 & 2.5915000000 \\ \mathrm{H} & -7.2159000000 & -0.6395000000 & 0.7583000000 \\ \mathrm{H} & -6.4856000000 & -0.4594000000 & -1.6118000000 \\ \mathrm{H} & -4.1689000000 & 0.2454000000 & -2.1524000000\end{array}$

Compound ID : I

Calculation : OPT + FREQ, Basis Set : TZVP, Functional : BP86

Imaginary Frequency $=1$ (TS)

Dipole Moment $=6.76$ debye

$\mathrm{EE}=-0.028389893$ hatree

$\begin{array}{lrrr}\mathrm{C} & -6.6218000000 & -4.0027000000 & 1.5178000000 \\ \mathrm{C} & -5.8629000000 & -3.2126000000 & 0.4862000000 \\ \mathrm{C} & -4.7922000000 & -3.7925000000 & -0.1918000000 \\ \mathrm{C} & -4.0780000000 & -3.0668000000 & -1.1323000000 \\ \mathrm{C} & -4.4345000000 & -1.7516000000 & -1.4078000000 \\ \mathrm{~N} & -3.7172000000 & -1.0372000000 & -2.4480000000 \\ \mathrm{C} & -2.7217000000 & -0.0091000000 & -2.2641000000 \\ \mathrm{O} & -2.0369000000 & 0.2047000000 & -3.2606000000 \\ \mathrm{C} & -2.5935000000 & 0.7793000000 & -0.9711000000 \\ \mathrm{C} & -3.1426000000 & 2.1992000000 & -1.2176000000 \\ \mathrm{C} & -2.2297000000 & 3.2680000000 & -1.7504000000 \\ \mathrm{C} & -2.2283000000 & 4.5167000000 & -1.1275000000 \\ \mathrm{C} & -1.4560000000 & 5.5565000000 & -1.6236000000 \\ \mathrm{C} & -0.6710000000 & 5.3632000000 & -2.7503000000\end{array}$




\begin{tabular}{|c|c|c|c|}
\hline $\mathrm{C}$ & -0.6591000000 & 4.1238000000 & -3.3750000000 \\
\hline $\mathrm{C}$ & -1.4296000000 & 3.0776000000 & -2.8854000000 \\
\hline $\mathrm{N}$ & -1.2213000000 & 0.5562000000 & -0.5360000000 \\
\hline $\mathrm{P}$ & -0.5950000000 & 0.7430000000 & 0.9880000000 \\
\hline $\mathrm{C}$ & -1.4353000000 & 1.9176000000 & 2.1030000000 \\
\hline $\mathrm{C}$ & -2.7555000000 & 1.6909000000 & 2.4913000000 \\
\hline $\mathrm{C}$ & -3.4241000000 & 2.5998000000 & 3.2955000000 \\
\hline $\mathrm{C}$ & -2.7804000000 & 3.7518000000 & 3.7242000000 \\
\hline $\mathrm{C}$ & -1.4688000000 & 3.9913000000 & 3.3439000000 \\
\hline $\mathrm{C}$ & -0.8040000000 & 3.0792000000 & 2.5379000000 \\
\hline $\mathrm{C}$ & 1.1196000000 & 1.3189000000 & 0.8090000000 \\
\hline $\mathrm{C}$ & 2.1689000000 & 0.9336000000 & 1.6423000000 \\
\hline $\mathrm{C}$ & 3.4543000000 & 1.4068000000 & 1.4357000000 \\
\hline $\mathrm{C}$ & 3.7148000000 & 2.2734000000 & 0.3851000000 \\
\hline $\mathrm{C}$ & 2.6833000000 & 2.6598000000 & -0.4560000000 \\
\hline $\mathrm{C}$ & 1.3972000000 & 2.1841000000 & -0.2438000000 \\
\hline $\mathrm{C}$ & -0.4880000000 & -0.8424000000 & 1.9540000000 \\
\hline $\mathrm{C}$ & -0.4978000000 & -2.1635000000 & 1.5190000000 \\
\hline $\mathrm{C}$ & -0.7388000000 & -2.6316000000 & 0.1073000000 \\
\hline $\mathrm{O}$ & 0.3661000000 & -2.2590000000 & -0.7144000000 \\
\hline $\mathrm{C}$ & 0.1843000000 & -2.3935000000 & -2.0534000000 \\
\hline $\mathrm{O}$ & -0.7254000000 & -3.0913000000 & -2.4507000000 \\
\hline $\mathrm{C}$ & 1.1574000000 & -1.6232000000 & -2.9035000000 \\
\hline $\mathrm{N}$ & 2.3816000000 & -1.1939000000 & -2.2412000000 \\
\hline $\mathrm{C}$ & 3.4082000000 & -2.0978000000 & -1.9909000000 \\
\hline $\mathrm{O}$ & 3.4859000000 & -3.2429000000 & -2.3939000000 \\
\hline $\mathrm{O}$ & 4.3847000000 & -1.4801000000 & -1.2731000000 \\
\hline $\mathrm{C}$ & 5.5663000000 & -2.2372000000 & -0.9308000000 \\
\hline $\mathrm{C}$ & 6.4594000000 & -1.3233000000 & -0.1379000000 \\
\hline $\mathrm{C}$ & 7.0445000000 & -0.2151000000 & -0.7402000000 \\
\hline $\mathrm{C}$ & 7.8819000000 & 0.6129000000 & -0.0086000000 \\
\hline $\mathrm{C}$ & 8.1427000000 & 0.3364000000 & 1.3257000000 \\
\hline $\mathrm{C}$ & 7.5629000000 & -0.7690000000 & 1.9283000000 \\
\hline $\mathrm{C}$ & 6.7204000000 & -1.5970000000 & 1.2002000000 \\
\hline $\mathrm{C}$ & -0.3486000000 & -3.2020000000 & 2.4460000000 \\
\hline $\mathrm{C}$ & -0.2003000000 & -2.9543000000 & 3.7950000000 \\
\hline $\mathrm{C}$ & -0.2020000000 & -1.6433000000 & 4.2455000000 \\
\hline $\mathrm{C}$ & -0.3417000000 & -0.6173000000 & 3.3327000000 \\
\hline $\mathrm{C}$ & -5.5067000000 & -1.1673000000 & -0.7465000000 \\
\hline $\mathrm{C}$ & -6.2132000000 & -1.8963000000 & 0.1997000000 \\
\hline $\mathrm{H}$ & -7.5991000000 & -3.5694000000 & 1.7404000000 \\
\hline $\mathrm{H}$ & -6.7886000000 & -5.0330000000 & 1.1946000000 \\
\hline $\mathrm{H}$ & -6.0619000000 & -4.0432000000 & 2.4566000000 \\
\hline $\mathrm{H}$ & -4.5192000000 & -4.8249000000 & 0.0184000000 \\
\hline $\mathrm{H}$ & -3.2393000000 & -3.5349000000 & -1.6547000000 \\
\hline $\mathrm{H}$ & -3.5568000000 & -1.6355000000 & -3.2310000000 \\
\hline
\end{tabular}




$\begin{array}{lrrr}\mathrm{H} & -3.2653000000 & 0.3349000000 & -0.1951000000 \\ \mathrm{H} & -4.0031000000 & 2.1438000000 & -1.9074000000 \\ \mathrm{H} & -3.5745000000 & 2.5394000000 & -0.2587000000 \\ \mathrm{H} & -2.8442000000 & 4.6823000000 & -0.2455000000 \\ \mathrm{H} & -1.4692000000 & 6.5257000000 & -1.1307000000 \\ \mathrm{H} & -0.0702000000 & 6.1801000000 & -3.1428000000 \\ \mathrm{H} & -0.0476000000 & 3.9697000000 & -4.2611000000 \\ \mathrm{H} & -1.3940000000 & 2.0998000000 & -3.3874000000 \\ \mathrm{H} & -3.2724000000 & 0.7891000000 & 2.1605000000 \\ \mathrm{H} & -4.4541000000 & 2.4112000000 & 3.5887000000 \\ \mathrm{H} & -3.3042000000 & 4.4662000000 & 4.3550000000 \\ \mathrm{H} & -0.9621000000 & 4.8941000000 & 3.6762000000 \\ \mathrm{H} & 0.2248000000 & 3.2733000000 & 2.2311000000 \\ \mathrm{H} & 1.9838000000 & 0.2441000000 & 2.4654000000 \\ \mathrm{H} & 4.2619000000 & 1.0896000000 & 2.0908000000 \\ \mathrm{H} & 4.7246000000 & 2.6407000000 & 0.2186000000 \\ \mathrm{H} & 2.8783000000 & 3.3392000000 & -1.2827000000 \\ \mathrm{H} & 0.5992000000 & 2.4876000000 & -0.9262000000 \\ \mathrm{H} & -1.7015000000 & -2.2420000000 & -0.2673000000 \\ \mathrm{H} & -0.8121000000 & -3.7318000000 & 0.0827000000 \\ \mathrm{H} & 0.6325000000 & -0.7210000000 & -3.2590000000 \\ \mathrm{H} & 1.4216000000 & -2.2102000000 & -3.7960000000 \\ \mathrm{H} & 2.3580000000 & -0.3238000000 & -1.7660000000 \\ \mathrm{H} & 6.0805000000 & -2.5753000000 & -1.8424000000 \\ \mathrm{H} & 5.2820000000 & -3.1291000000 & -0.3541000000 \\ \mathrm{H} & 6.8430000000 & 0.0005000000 & -1.7873000000 \\ \mathrm{H} & 8.3377000000 & 1.4781000000 & -0.4841000000 \\ \mathrm{H} & 8.8025000000 & 0.9843000000 & 1.8979000000 \\ \mathrm{H} & 7.7689000000 & -0.9879000000 & 2.9733000000 \\ \mathrm{H} & 6.2654000000 & -2.4632000000 & 1.6771000000 \\ \mathrm{H} & -0.3524000000 & -4.2328000000 & 2.0909000000 \\ \mathrm{H} & -0.0869000000 & -3.7764000000 & 4.4974000000 \\ \mathrm{H} & -0.0925000000 & -1.4275000000 & 5.3053000000 \\ \mathrm{H} & -0.3471000000 & 0.4090000000 & 3.7061000000 \\ \mathrm{H} & -5.8034000000 & -0.1438000000 & -0.9705000000 \\ & -7.0533000000 & -1.4371000000 & 0.7170000000\end{array}$

Compound ID : II

Calculation : OPT + FREQ, Basis Set : TZVP, Functional : BP86

Imaginary Frequency $=1$ (TS)

Dipole Moment $=14.022$ debye

$\begin{array}{llll}\text { C } & -6.3287940000 & 5.1693060000 & -2.2797660000 \\ \text { C } & -5.4074010000 & 4.0649220000 & -1.8537960000 \\ \text { C } & -4.3882280000 & 4.3290020000 & -0.9270760000 \\ \text { C } & -3.5435920000 & 3.3025620000 & -0.4955690000\end{array}$




\begin{tabular}{|c|c|c|c|}
\hline & -3.7011420000 & 2.0022130000 & \\
\hline 1 & -2.9048510000 & 0.9392830000 & \\
\hline & -2.0652730000 & 0.2723750000 & \\
\hline ) & -1.8971200000 & 0.7659150000 & \\
\hline & -1.3962590000 & -1.0770680000 & \\
\hline & -2.4593610000 & -2.0181410000 & \\
\hline & -3.6459540000 & -2.2291250000 & \\
\hline 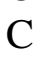 & -3.4692190000 & -2.7668250000 & \\
\hline $\mathrm{C}$ & -4.5640540000 & -2.9376890000 & \\
\hline & -5.8476670000 & -2.5970900000 & \\
\hline ( & -6.0407040000 & 4130000 & \\
\hline & 5960000 & 50000 & \\
\hline $\mathrm{N}$ & 90000 & 70000 & \\
\hline & 0.79 & -2.43 & 0.5699480 \\
\hline $\mathrm{C}$ & 970000 & 1950000 & 2.2278330 \\
\hline C & 0.31 & 20000 & \\
\hline $\mathrm{C}$ & 70000 & 9990000 & 4.6 \\
\hline & -1.23 & 2060000 & \\
\hline $\mathrm{C}$ & 50000 & 520000 & 3.6142260 \\
\hline ( & -1.0 & 50000 & \\
\hline $\mathrm{C}$ & 300000 & -2.2105140000 & 1.68162800 \\
\hline C & 2.523 & & \\
\hline $\mathrm{C}$ & 3.62 & $-0.9^{\prime}$ & \\
\hline $\mathrm{C}$ & 30000 & 5680000 & \\
\hline $\mathrm{C}$ & 4.6 & -2.9 & \\
\hline & 3.48 & 20000 & \\
\hline $\mathrm{C}$ & 2.27 & -2.3 & -0.71781 \\
\hline C & 3.05 & 490000 & -0.92979 \\
\hline $\mathrm{C}$ & 2.90 & 90000 & -0.212925 \\
\hline $\mathrm{O}$ & 1.7 & 110000 & \\
\hline $\mathrm{C}$ & 0.5 & 940000 & \\
\hline $\mathrm{O}$ & 0.7 & 70000 & -1.431078000 \\
\hline $\mathrm{C}$ & -0.19 & 0980000 & 0.529364000 \\
\hline $\mathrm{N}$ & 0.6 & 10000 & 1.290492000 \\
\hline $\mathrm{C}$ & 1.62 & 280000 & 0.461912000 \\
\hline $\mathrm{O}$ & 310000 & 3.9783760000 & -0.439924000 \\
\hline $\mathrm{O}$ & 2.97 & 51700000 & 0.767928000 \\
\hline $\mathrm{C}$ & 3.82 & 3.6747020000 & -0.221653000 \\
\hline $\mathrm{C}$ & 9060000 & 3.2207670000 & -0.030372000 \\
\hline $\mathrm{C}$ & 6.21 & 1670000 & -0.988943000 \\
\hline $\mathrm{C}$ & 0290000 & 3.1572640000 & -0.860373000 \\
\hline $\mathrm{C}$ & 7.9175230000 & 2.3686850000 & 0.227936000 \\
\hline $\mathrm{C}$ & 6.9735440000 & 2.0068910000 & 1.191391000 \\
\hline $\mathrm{C}$ & 5.6468960000 & 2.4304470000 & \\
\hline $\mathrm{C}$ & 4.1118930000 & -1.0985510000 & \\
\hline $\mathrm{C}$ & 4.3669970000 & -2.1975340000 & -2.65040700 \\
\hline
\end{tabular}




\begin{tabular}{|c|c|c|c|}
\hline & .5690980000 & -3.3705650000 & \\
\hline & 2.5807140000 & -3.3723190000 & -1.6276650000 \\
\hline & -4.7198220000 & 1.7361180000 & -1.9217290000 \\
\hline & -5.5640360000 & 2.7625800000 & -2.3545870000 \\
\hline & -6.8614420000 & 5.5721450000 & \\
\hline & -5.7416390000 & 5.9849390000 & \\
\hline & -7.0851120000 & 4.8106200000 & \\
\hline & -4.2610550000 & 5.3263890000 & 3873000 \\
\hline & -2.7857690000 & & \\
\hline & -3.5755330000 & 0.2691390000 & -0.0 \\
\hline & 140000 & 77340000 & \\
\hline & -2.8102550000 & 776800000 & \\
\hline & -1.9980030000 & -2.9 & \\
\hline & -2.4872720000 & 5760000 & \\
\hline & 0650000 & 364440000 & -4.6 \\
\hline I & -6.69 & -2.7 & \\
\hline & -7.0 & -1.8 & -1.5 \\
\hline & -5.1 & & \\
\hline & 1.09 & -3.5 & \\
\hline & 0.34 & -2.9 & \\
\hline H & -1.70 & -1.3 & \\
\hline & -2.39 & & \\
\hline H & -1.44 & -0.5 & 7040000 \\
\hline & 1.73 & -0.4 & 4990000 \\
\hline $\mathrm{H}$ & 3.67 & -0.1 & 5870000 \\
\hline & 5.56 & -1.7 & 3.9 \\
\hline $\mathrm{H}$ & 5.44 & -3.6 & \\
\hline & 3.53 & -3.9 & 4070000 \\
\hline & & & \\
\hline $\mathrm{H}$ & 3.76 & & \\
\hline $\mathrm{H}$ & -0.75 & 2.2511390000 & 31770000 \\
\hline $\mathrm{H}$ & -0.96 & & 1420000 \\
\hline & 1.15 & & \\
\hline $\mathrm{H}$ & $3.78 \mathrm{C}$ & 4.7 & -0.1 \\
\hline & 3.506 & 3.356393000 & -1.2 \\
\hline $\mathrm{H}$ & 5.930 & 4.18 & 22450000 \\
\hline $\mathrm{H}$ & $8.26 \mathrm{C}$ & 3.4363790000 & -1.6068700000 \\
\hline & 8.943 & & \\
\hline $\mathrm{H}$ & 7.2695130000 & 1.3966480000 & 2.0351990000 \\
\hline $\mathrm{H}$ & 4.939 & 2.1344180000 & 1.8320890000 \\
\hline $\mathrm{H}$ & 4.7340240000 & -0.2148730000 & -1.9902700000 \\
\hline & 5.191 & -2.1342320000 & -3.3624050000 \\
\hline $\mathrm{H}$ & 4.0707180000 & -4.0818130000 & -3.1991910000 \\
\hline & 2.0713290000 & -4.3037910000 & -1.6096920000 \\
\hline $\mathrm{H}$ & -4.8678730000 & 0.7314960000 & -2.2960540000 \\
\hline & -6.3490100000 & 2.5363940000 & -3.0654740000 \\
\hline
\end{tabular}




$\begin{array}{lrrr}\mathrm{N} & 0.1706050000 & -4.2399030000 & 0.3939600000 \\ \mathrm{C} & 1.3462930000 & -5.1433420000 & 0.7245300000 \\ \mathrm{C} & 1.2072160000 & -6.6594890000 & 0.4453300000 \\ \mathrm{C} & -0.8623470000 & -4.7640690000 & 1.4122180000 \\ \mathrm{C} & -2.2843440000 & -4.2125870000 & 1.5983660000 \\ \mathrm{C} & -0.2482410000 & -4.5097850000 & -1.0403520000 \\ \mathrm{C} & -1.3810040000 & -5.5231710000 & -1.3221650000 \\ \mathrm{H} & 0.4040120000 & -1.2163500000 & -1.3473910000 \\ \mathrm{H} & -1.1710750000 & -6.5256200000 & -0.9037870000 \\ \mathrm{H} & 2.2520560000 & -4.8741060000 & 0.1913410000 \\ \mathrm{H} & 1.6274830000 & -5.0459590000 & 1.7948240000 \\ \mathrm{H} & 0.3663540000 & -7.1211020000 & 0.9977150000 \\ \mathrm{H} & 2.1301870000 & -7.1691800000 & 0.7951240000 \\ \mathrm{H} & 1.1196310000 & -6.8754120000 & -0.6376720000 \\ \mathrm{H} & -1.0766280000 & -5.8298080000 & 1.2080960000 \\ \mathrm{H} & -0.4409610000 & -4.8582790000 & 2.4261650000 \\ \mathrm{H} & -2.7874680000 & -4.8231550000 & 2.3791050000 \\ \mathrm{H} & -2.3256150000 & -3.1936560000 & 1.9867530000 \\ \mathrm{H} & -2.9164630000 & -4.3304190000 & 0.7021390000 \\ \mathrm{H} & 0.5944520000 & -4.9006110000 & -1.6358770000 \\ \mathrm{H} & -0.4940740000 & -3.5764000000 & -1.5657630000 \\ \mathrm{H} & -1.4730070000 & -5.6513570000 & -2.4216710000 \\ \mathrm{H} & -2.3660680000 & -5.1702140000 & -0.9741910000\end{array}$

Compound ID : III

Calculation : OPT + FREQ, Basis Set : TZVP, Functional : BP86

Imaginary Frequency $=1$ (TS)

Dipole Moment $=11.87$ debye

$\mathrm{EE}=-0.04626794$ hatree

$\begin{array}{lrrr}\mathrm{C} & 8.3182000000 & 2.6897000000 & 1.1205000000 \\ \mathrm{C} & 8.6847000000 & 2.0850000000 & -0.0773000000 \\ \mathrm{C} & 7.8508000000 & 1.1368000000 & -0.6615000000 \\ \mathrm{C} & 6.6520000000 & 0.7915000000 & -0.0487000000 \\ \mathrm{C} & 6.2832000000 & 1.3964000000 & 1.1535000000 \\ \mathrm{C} & 7.1189000000 & 2.3488000000 & 1.7365000000 \\ \mathrm{C} & 4.9980000000 & 1.0095000000 & 1.8222000000 \\ \mathrm{O} & 3.9734000000 & 1.7962000000 & 1.1982000000 \\ \mathrm{C} & 2.6856000000 & 1.5189000000 & 1.5286000000 \\ \mathrm{~N} & 1.7975000000 & 2.4079000000 & 0.8549000000 \\ \mathrm{O} & 2.3723000000 & 0.6670000000 & 2.3492000000 \\ \mathrm{C} & 0.3758000000 & 2.0199000000 & 0.7684000000 \\ \mathrm{C} & 0.0820000000 & 1.0422000000 & -0.3748000000 \\ \mathrm{O} & 0.4222000000 & 1.2830000000 & -1.5128000000 \\ \mathrm{~N} & -0.5842000000 & -0.2283000000 & -0.1004000000\end{array}$




\begin{tabular}{|c|c|c|c|}
\hline & -1.8998000000 & -0.1715000000 & \\
\hline & -2.8529000000 & 0.8903000000 & 0.0215000000 \\
\hline & -3.0091000000 & 2.1447000000 & 0.6933000000 \\
\hline & -3.5391000000 & 0.6542000000 & -0.9547000000 \\
\hline & -3.3017000000 & 3.3567000000 & \\
\hline & -4.5497000000 & 3.5300000000 & 67000000 \\
\hline & -4.8340000000 & 4.7257000000 & -1.2966000000 \\
\hline & -3.8896000000 & 5.7515000000 & 54000000 \\
\hline & -2.6448000000 & 5.5709000000 & -0.7389000000 \\
\hline & -2.3462000000 & 4.3798000000 & -0.0937000000 \\
\hline C & 71000000 & 194000000 & 3000000 \\
\hline & 75000000 & -1.8000000000 & -0.88 \\
\hline $\mathrm{C}$ & 70000000 & 41000000 & \\
\hline E & -2.1554000000 & -2.1355000000 & -2.7948000000 \\
\hline C & 5000000 & -2.7752000000 & -3.2035000000 \\
\hline C & 2000000 & 5000000 & -2.3 \\
\hline C & 2000000 & 0000000 & -1.1 \\
\hline & -2.2 & 9000000 & \\
\hline C & -1.5 & 53000000 & -3.8086000000 \\
\hline $\mathrm{O}$ & -0.7 & -1.7 & \\
\hline $\mathrm{C}$ & -1.7 & -0.03 & \\
\hline $\mathrm{C}$ & -2.9 & -0.45 & \\
\hline $\mathrm{C}$ & -3.7 & & \\
\hline C & 000000 & 5000000 & 440000000 \\
\hline $\mathrm{C}$ & -5.1 & -1.3 & \\
\hline C & 000000 & -2.2 & 3.6 \\
\hline $\mathrm{C}$ & -3.2 & -1.8 & 2.9002000 \\
\hline Y & 0.40 & -2.8 & \\
\hline $\mathrm{C}$ & 0.5 & -4.2 & \\
\hline $\mathrm{C}$ & 1.0893000000 & & \\
\hline $\mathrm{C}$ & 1.4079000000 & & 15000000 \\
\hline $\mathrm{C}$ & 000000 & -3.3 & 2.7039000000 \\
\hline $\mathrm{C}$ & 000000 & -2.46 & 1.7667000000 \\
\hline $\mathrm{C}$ & 1.38 & -1.5 & -1.73 \\
\hline $\mathrm{C}$ & 2.51 & 15000000 & -0.9578000 \\
\hline $\mathrm{C}$ & 2000000 & 14000000 & -1.5419000000 \\
\hline $\mathrm{C}$ & 3.9014000000 & -1.3689000000 & -2.9189000 \\
\hline $\mathrm{C}$ & 000000 & -1.6768000000 & -3.6837000 \\
\hline $\mathrm{C}$ & 1.5221000000 & -1.7867000000 & -3.1009000000 \\
\hline $\mathrm{H}$ & 000000 & 73000000 & 1.5796000000 \\
\hline $\mathrm{H}$ & 9.6289000000 & 2.3560000000 & -0.5612000000 \\
\hline $\mathrm{H}$ & & & -1.6061000000 \\
\hline $\mathrm{H}$ & 5.9879000000 & 0.0445000000 & -0.512400000 \\
\hline $\mathrm{H}$ & & 2.8288000000 & 2.678700000 \\
\hline $\mathrm{H}$ & 4.7831000000 & -0.0687000000 & 1.6840000000 \\
\hline 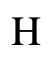 & 5.0111000000 & 1.2239000000 & 2.9075000000 \\
\hline
\end{tabular}




$\begin{array}{lrrr}\mathrm{H} & 2.1513000000 & 2.7968000000 & 0.0051000000 \\ \mathrm{H} & 0.0463000000 & 1.6254000000 & 1.7569000000 \\ \mathrm{H} & -0.1949000000 & 2.9656000000 & 0.5935000000 \\ \mathrm{H} & -2.3750000000 & -1.1720000000 & 0.4266000000 \\ \mathrm{H} & -2.3160000000 & 2.2895000000 & 1.4033000000 \\ \mathrm{H} & -5.2968000000 & 2.7273000000 & -0.6269000000 \\ \mathrm{H} & -5.8104000000 & 4.8617000000 & -1.7761000000 \\ \mathrm{H} & -1.8981000000 & 6.3721000000 & -0.7780000000 \\ \mathrm{H} & -1.3542000000 & 4.2443000000 & 0.3669000000 \\ \mathrm{H} & -3.8962000000 & 6.9485000000 & -3.1108000000 \\ \mathrm{H} & -5.27550000000 & 7.8733000000 & -1.6171000000 \\ \mathrm{H} & -3.7497000000 & -2.5282000000 & -4.1940000000 \\ \mathrm{H} & -4.9054000000 & -4.1812000000 & -2.7282000000 \\ \mathrm{H} & -3.9629000000 & -4.6962000000 & -0.4776000000 \\ \mathrm{H} & -1.8880000000 & -3.6238000000 & 0.2646000000 \\ \mathrm{H} & -2.3929000000 & -0.6044000000 & -4.3002000000 \\ \mathrm{H} & -1.0059000000 & -0.3673000000 & -3.2035000000 \\ \mathrm{H} & -1.4641000000 & 1.0137000000 & 2.4252000000 \\ \mathrm{H} & -0.8535000000 & -0.6431000000 & 2.4901000000 \\ \mathrm{H} & -3.4377000000 & 1.4730000000 & 3.6366000000 \\ \mathrm{H} & -5.4121000000 & 0.6879000000 & 4.9116000000 \\ \mathrm{H} & -6.0327000000 & -1.7208000000 & 4.8999000000 \\ \mathrm{H} & -4.6627000000 & -3.3474000000 & 3.6099000000 \\ \mathrm{H} & -2.6807000000 & -2.5745000000 & 2.3363000000 \\ \mathrm{H} & 0.3477000000 & -4.5677000000 & -0.8673000000 \\ \mathrm{H} & 1.2321000000 & -6.1776000000 & 0.7953000000 \\ \mathrm{H} & 1.7996000000 & -5.4095000000 & 3.0975000000 \\ \mathrm{H} & 1.4900000000 & -3.0138000000 & 3.7098000000 \\ \mathrm{H} & 0.6200000000 & -1.4042000000 & 2.0619000000 \\ \mathrm{H} & 2.4138000000 & -1.1592000000 & 0.1297000000 \\ \mathrm{H} & 4.6353000000 & -0.9142000000 & -0.9289000000 \\ \mathrm{H} & 4.8895000000 & -1.2741000000 & -3.3814000000 \\ & 2.8849000000 & -1.8259000000 & -4.7659000000 \\ & 0.6859590100 & -1.9579199400 & -3.7626794000\end{array}$

Compound ID : $\mathbf{2} \mathbf{a}^{\prime}$

Calculation : OPT + FREQ, Basis Set : TZVP, Functional : BP86

Imaginary Frequency $=0$

Dipole Moment $=3.43$ debye

$\mathrm{EE}=-0.012082767$ hatree

$\begin{array}{rrrr}\mathrm{O} & 0.8705000000 & 0.0967000000 & 2.7960000000 \\ \mathrm{C} & 1.1606000000 & -0.9613000000 & 1.9119000000 \\ \mathrm{C} & 2.0442000000 & -0.5229000000 & 0.7699000000 \\ \mathrm{C} & 1.5675000000 & -0.1134000000 & -0.4782000000\end{array}$




$\begin{array}{lrrr}\mathrm{C} & 2.5047000000 & 0.2796000000 & -1.4362000000 \\ \mathrm{C} & 3.8704000000 & 0.2717000000 & -1.1803000000 \\ \mathrm{C} & 4.3331000000 & -0.1382000000 & 0.0644000000 \\ \mathrm{C} & 3.4199000000 & -0.5327000000 & 1.0313000000 \\ \mathrm{P} & -0.1703000000 & 0.0334000000 & -1.1386000000 \\ \mathrm{O} & -0.2032000000 & 0.0496000000 & -2.6194000000 \\ \mathrm{C} & -0.8386000000 & 1.5424000000 & -0.3743000000 \\ \mathrm{C} & -0.6793000000 & 1.8726000000 & 0.9688000000 \\ \mathrm{C} & -1.2183000000 & 3.0518000000 & 1.4755000000 \\ \mathrm{C} & -1.9217000000 & 3.9142000000 & 0.6419000000 \\ \mathrm{C} & -2.0836000000 & 3.5936000000 & -0.7016000000 \\ \mathrm{C} & -1.5434000000 & 2.4131000000 & -1.2023000000 \\ \mathrm{C} & -1.1200000000 & -1.3635000000 & -0.4526000000 \\ \mathrm{C} & -0.9008000000 & -2.6281000000 & -0.9921000000 \\ \mathrm{C} & -1.6410000000 & -3.7245000000 & -0.5594000000 \\ \mathrm{C} & -2.6155000000 & -3.5608000000 & 0.4193000000 \\ \mathrm{C} & -2.8458000000 & -2.3005000000 & 0.9613000000 \\ \mathrm{C} & -2.0998000000 & -1.2104000000 & 0.5240000000 \\ \mathrm{H} & 1.7000000000 & 0.4541000000 & 3.0916000000 \\ \mathrm{H} & 1.6270000000 & -1.8011000000 & 2.4650000000 \\ \mathrm{H} & 0.1588000000 & -1.2959000000 & 1.5838000000 \\ \mathrm{H} & 2.1581000000 & 0.6000000000 & -2.4301000000 \\ \mathrm{H} & 4.5775000000 & 0.5850000000 & -1.9555000000 \\ \mathrm{H} & 5.4062000000 & -0.1513000000 & 0.2804000000 \\ \mathrm{H} & 3.7840000000 & -0.8621000000 & 2.0122000000 \\ \mathrm{H} & -0.1145000000 & 1.2012000000 & 1.6540000000 \\ \mathrm{H} & -1.0865000000 & 3.2991000000 & 2.5340000000 \\ \mathrm{H} & -2.3451000000 & 4.8417000000 & 1.0408000000 \\ \mathrm{H} & -2.6342000000 & 4.2686000000 & -1.3648000000 \\ \mathrm{H} & -1.6698000000 & 2.1667000000 & -2.2656000000 \\ \mathrm{H} & -0.1443000000 & -2.7588000000 & -1.7762000000 \\ \mathrm{H} & -1.4599000000 & -4.7135000000 & -0.9930000000 \\ \mathrm{H} & -3.2017000000 & -4.4205000000 & 0.7602000000 \\ & -3.6140000000 & -2.1653000000 & 1.7296000000 \\ & -2.2807000000 & -0.2147000000 & 0.9539000000\end{array}$

Compound ID : 6aa

Calculation : OPT + FREQ, Basis Set : TZVP, Functional : BP86

Imaginary Frequency $=0$

Dipole Moment $=2.24$ debye

$\mathrm{EE}=-0.1455841$ hatree

$\begin{array}{lrrr}\mathrm{C} & 9.5303000000 & 0.4120000000 & 0.9386000000 \\ \mathrm{C} & 9.9128000000 & 1.0528000000 & -0.2353000000 \\ \mathrm{C} & 9.2146000000 & 0.8171000000 & -1.4150000000 \\ \mathrm{C} & 8.1346000000 & -0.0586000000 & -1.4235000000\end{array}$




\begin{tabular}{|c|c|c|c|}
\hline $\mathrm{C}$ & 7.7498000000 & -0.7029000000 & -0.2473000000 \\
\hline $\mathrm{C}$ & 8.4505000000 & -0.4641000000 & 0.9352000000 \\
\hline $\mathrm{C}$ & 6.5946000000 & -1.6601000000 & -0.2573000000 \\
\hline $\mathrm{O}$ & 5.4231000000 & -0.8687000000 & -0.0211000000 \\
\hline $\mathrm{C}$ & 4.2264000000 & -1.5178000000 & 0.0005000000 \\
\hline $\mathrm{N}$ & 3.1604000000 & -0.5762000000 & 0.0787000000 \\
\hline $\mathrm{O}$ & 4.1195000000 & -2.7300000000 & -0.1043000000 \\
\hline $\mathrm{C}$ & 1.8484000000 & -1.1105000000 & 0.5188000000 \\
\hline $\mathrm{C}$ & 0.8243000000 & 0.0141000000 & 0.6138000000 \\
\hline $\mathrm{O}$ & 1.0447000000 & 1.0777000000 & 1.1742000000 \\
\hline $\mathrm{N}$ & -0.4200000000 & -0.1926000000 & -0.0397000000 \\
\hline $\mathrm{C}$ & -1.6049000000 & 0.6488000000 & 0.2992000000 \\
\hline $\mathrm{C}$ & -2.8017000000 & -0.2686000000 & 0.6193000000 \\
\hline $\mathrm{N}$ & -3.4933000000 & -0.8536000000 & -0.4854000000 \\
\hline $\mathrm{O}$ & -3.0933000000 & -0.5634000000 & 1.7654000000 \\
\hline $\mathrm{C}$ & -4.8126000000 & -1.4302000000 & -0.3400000000 \\
\hline $\mathrm{C}$ & -5.0147000000 & -2.5313000000 & 0.4974000000 \\
\hline $\mathrm{C}$ & -6.2728000000 & -3.1171000000 & 0.5686000000 \\
\hline $\mathrm{C}$ & -7.3329000000 & -2.6215000000 & -0.1913000000 \\
\hline $\mathrm{C}$ & -7.1225000000 & -1.5249000000 & -1.0295000000 \\
\hline $\mathrm{C}$ & -5.8725000000 & -0.9268000000 & -1.1049000000 \\
\hline $\mathrm{C}$ & -8.6777000000 & -3.2453000000 & -0.0987000000 \\
\hline $\mathrm{C}$ & -1.8845000000 & 1.6261000000 & -0.8563000000 \\
\hline $\mathrm{C}$ & -2.8055000000 & 2.7263000000 & -0.4355000000 \\
\hline $\mathrm{C}$ & -4.1086000000 & 2.7894000000 & -0.9300000000 \\
\hline $\mathrm{C}$ & -4.9513000000 & 3.8276000000 & -0.5481000000 \\
\hline $\mathrm{C}$ & -4.4987000000 & 4.8072000000 & 0.3287000000 \\
\hline $\mathrm{C}$ & -3.1991000000 & 4.7493000000 & 0.8222000000 \\
\hline $\mathrm{C}$ & -2.3529000000 & 3.7147000000 & 0.4414000000 \\
\hline $\mathrm{H}$ & 10.0789000000 & 0.5979000000 & 1.8678000000 \\
\hline $\mathrm{H}$ & 10.7630000000 & 1.7427000000 & -0.2305000000 \\
\hline $\mathrm{H}$ & 9.5145000000 & 1.3221000000 & -2.3391000000 \\
\hline $\mathrm{H}$ & 7.5838000000 & -0.2423000000 & -2.3530000000 \\
\hline $\mathrm{H}$ & 8.1494000000 & -0.9668000000 & 1.8613000000 \\
\hline $\mathrm{H}$ & 6.5043000000 & -2.1803000000 & -1.2301000000 \\
\hline $\mathrm{H}$ & 6.6915000000 & -2.4276000000 & 0.5344000000 \\
\hline $\mathrm{H}$ & 3.3980000000 & 0.2964000000 & 0.5043000000 \\
\hline $\mathrm{H}$ & 1.5528000000 & -1.8953000000 & -0.2094000000 \\
\hline $\mathrm{H}$ & 1.9101000000 & -1.6134000000 & 1.5086000000 \\
\hline $\mathrm{H}$ & -0.6339000000 & -1.1467000000 & -0.2361000000 \\
\hline $\mathrm{H}$ & -1.3845000000 & 1.2594000000 & 1.2171000000 \\
\hline $\mathrm{H}$ & -3.4111000000 & -0.2952000000 & -1.3151000000 \\
\hline $\mathrm{H}$ & -4.1960000000 & -2.9283000000 & 1.1092000000 \\
\hline $\mathrm{H}$ & -6.4324000000 & -3.9779000000 & 1.2282000000 \\
\hline $\mathrm{H}$ & -7.9508000000 & -1.1321000000 & -1.6302000000 \\
\hline $\mathrm{H}$ & -5.7292000000 & -0.0620000000 & -1.7658000000 \\
\hline
\end{tabular}




$\begin{array}{lrrr}\text { H } & -9.2399000000 & -3.1453000000 & -1.0366000000 \\ \mathrm{H} & -9.2680000000 & -2.7647000000 & 0.6937000000 \\ \mathrm{H} & -8.6156000000 & -4.3156000000 & 0.1388000000 \\ \mathrm{H} & -0.9318000000 & 2.0763000000 & -1.2038000000 \\ \mathrm{H} & -2.2996000000 & 1.0882000000 & -1.7381000000 \\ \mathrm{H} & -4.4738000000 & 2.0211000000 & -1.6230000000 \\ \mathrm{H} & -5.9734000000 & 3.8715000000 & -0.9384000000 \\ \mathrm{H} & -5.1638000000 & 5.6229000000 & 0.6302000000 \\ \mathrm{H} & -2.8403000000 & 5.5202000000 & 1.5123000000 \\ \mathrm{H} & -1.3270000000 & 3.6723000000 & 0.8279000000\end{array}$

Table 1.

\begin{tabular}{lrrrrr}
\hline Entry & dG (kcal/mol) & dG (Hatree) & $\begin{array}{c}\text { dH } \\
\text { (kcal/mol) }\end{array}$ & dH(Hatree) & $\begin{array}{c}\text { Zero-point E } \\
\text { correction } \\
\text { (Hatree) }\end{array}$ \\
\hline 4a & 459.17 & 0.812505 & 578.39 & 0.921721 & 0.475771 \\
5a & 350.09 & 0.651878 & 387.09 & 0.616864 & 0.292100 \\
I & 877.09 & 1.397738 & 968.65 & 1.54364 & 0.760226 \\
II & 563.99 & 0.898788 & 639.77 & 1.019552 & 0.973231 \\
IV & 928.02 & 1.47889 & 1016.60 & 1.620046 & 0.761600 \\
2a' & 380.20 & 0.60588 & 389.43 & 0.6206 & 0.360550 \\
6aa & 463.33 & 0.738368 & 531.71 & 0.84734 & 0.48 \\
\hline
\end{tabular}

Computational data of Figure 2:

Compound ID : $\mathbf{5 b}$

Calculation : OPT + FREQ, Basis Set : TZVP, Functional : BP86

Imaginary Frequency $=0$

Dipole Moment $=3.25$ debye

$\mathrm{EE}=0.082565$ hatree

$\begin{array}{lrrr}\mathrm{C} & 2.8600000000 & 0.9154000000 & 0.6853000000 \\ \mathrm{C} & 1.5143000000 & 0.2036000000 & 0.5303000000 \\ \mathrm{~N} & 0.3499000000 & 1.0056000000 & 0.6737000000 \\ \mathrm{O} & 1.4358000000 & -1.0066000000 & 0.3739000000 \\ \mathrm{C} & -0.9716000000 & 0.5305000000 & 0.3284000000 \\ \mathrm{C} & -1.4914000000 & -0.6242000000 & 0.9217000000 \\ \mathrm{C} & -2.7898000000 & -1.0224000000 & 0.6276000000 \\ \mathrm{C} & -3.5829000000 & -0.2772000000 & -0.2455000000 \\ \mathrm{C} & -3.0595000000 & 0.8774000000 & -0.8305000000 \\ \mathrm{C} & -1.7622000000 & 1.2828000000 & -0.5499000000 \\ \mathrm{C} & -4.9755000000 & -0.7006000000 & -0.5410000000 \\ \mathrm{~N} & 3.7765000000 & 0.6506000000 & -0.4279000000 \\ \mathrm{~N} & 4.2761000000 & -0.4969000000 & -0.6395000000 \\ \mathrm{~N} & 4.8379000000 & -1.4110000000 & -0.9775000000\end{array}$




$\begin{array}{crrr}\mathrm{H} & 2.7747000000 & 2.0185000000 & 0.6487000000 \\ \mathrm{H} & 3.2982000000 & 0.6555000000 & 1.6719000000 \\ \mathrm{H} & 0.4912000000 & 1.9602000000 & 0.4209000000 \\ \mathrm{H} & -0.8823000000 & -1.2278000000 & 1.6051000000 \\ \mathrm{H} & -3.1935000000 & -1.9322000000 & 1.0868000000 \\ \mathrm{H} & -3.6762000000 & 1.4661000000 & -1.5192000000 \\ \mathrm{H} & -1.3730000000 & 2.1938000000 & -1.0210000000 \\ \mathrm{H} & -5.0942000000 & -1.7901000000 & -0.4713000000 \\ \mathrm{H} & -5.2897000000 & -0.3962000000 & -1.5481000000 \\ \mathrm{H} & -5.6738000000 & -0.2455000000 & 0.1750000000\end{array}$

Compound ID : 4d

Calculation : OPT + FREQ, Basis Set : TZVP, Functional : BP86

Imaginary Frequency $=0$

Dipole Moment $=3.06$ debye

$\mathrm{EE}=-0.226438$ hatree

$\begin{array}{lrrr}\mathrm{C} & -3.8942000000 & 2.5821000000 & -2.2038000000 \\ \mathrm{C} & -2.5162000000 & 2.9085000000 & -1.6238000000 \\ \mathrm{O} & -2.0153000000 & 1.8453000000 & -0.7751000000 \\ \mathrm{C} & -1.8918000000 & 0.5702000000 & -1.2285000000 \\ \mathrm{~N} & -1.7079000000 & -0.2722000000 & -0.0858000000 \\ \mathrm{O} & -1.9755000000 & 0.2233000000 & -2.3945000000 \\ \mathrm{C} & -1.4478000000 & -1.7273000000 & -0.2518000000 \\ \mathrm{C} & -0.1919000000 & -2.0534000000 & -1.0760000000 \\ \mathrm{O} & -0.0342000000 & -2.9630000000 & -1.8626000000 \\ \mathrm{C} & -2.6873000000 & -2.4534000000 & -0.7977000000 \\ \mathrm{C} & -1.5235000000 & 3.2571000000 & -2.7338000000 \\ \mathrm{C} & -2.6340000000 & 4.0871000000 & -0.6452000000 \\ \mathrm{C} & -3.7240000000 & -2.7595000000 & 0.2738000000 \\ \mathrm{C} & -4.4023000000 & -1.5377000000 & 0.8593000000 \\ \mathrm{O} & -4.3998000000 & -1.2027000000 & 2.0281000000 \\ \mathrm{O} & -5.0794000000 & -0.7963000000 & -0.0647000000 \\ \mathrm{C} & -5.7209000000 & 0.4155000000 & 0.3212000000 \\ \mathrm{C} & -7.1770000000 & 0.1934000000 & 0.6102000000 \\ \mathrm{C} & -7.6174000000 & 0.0538000000 & 1.9265000000 \\ \mathrm{C} & -8.9693000000 & -0.1369000000 & 2.1905000000 \\ \mathrm{C} & -9.8846000000 & -0.1897000000 & 1.1445000000 \\ \mathrm{C} & -9.4473000000 & -0.0526000000 & -0.1689000000 \\ \mathrm{C} & -8.0966000000 & 0.1387000000 & -0.4375000000 \\ \mathrm{O} & 0.8398000000 & -1.2034000000 & -0.7972000000 \\ \mathrm{C} & 2.0942000000 & -1.3317000000 & -1.4614000000 \\ \mathrm{C} & 3.1061000000 & -2.0520000000 & -0.6105000000 \\ \mathrm{C} & 4.2044000000 & -1.3790000000 & -0.0693000000 \\ \mathrm{C} & 5.1255000000 & -2.0837000000 & 0.6977000000 \\ \mathrm{C} & 4.9710000000 & -3.4463000000 & 0.9286000000\end{array}$




\begin{tabular}{|c|c|c|c|}
\hline $\mathrm{C}$ & 3.8845000000 & -4.1179000000 & 0.3797000000 \\
\hline $\mathrm{C}$ & 2.9588000000 & -3.4240000000 & -0.3901000000 \\
\hline $\mathrm{P}$ & 4.4497000000 & 0.4477000000 & -0.4340000000 \\
\hline $\mathrm{C}$ & 3.8569000000 & 1.2434000000 & 1.1338000000 \\
\hline $\mathrm{C}$ & 6.2931000000 & 0.6223000000 & -0.3101000000 \\
\hline $\mathrm{C}$ & 3.3288000000 & 2.5173000000 & 0.9593000000 \\
\hline $\mathrm{C}$ & 2.8241000000 & 3.2203000000 & 2.0493000000 \\
\hline $\mathrm{C}$ & 2.8461000000 & 2.6446000000 & 3.3160000000 \\
\hline $\mathrm{C}$ & 3.3727000000 & 1.3688000000 & 3.4910000000 \\
\hline $\mathrm{C}$ & 3.8793000000 & 0.6702000000 & 2.3987000000 \\
\hline $\mathrm{C}$ & 6.9579000000 & 0.5706000000 & -1.5298000000 \\
\hline $\mathrm{C}$ & 8.3389000000 & 0.7355000000 & -1.5809000000 \\
\hline $\mathrm{C}$ & 9.0548000000 & 0.9565000000 & -0.4081000000 \\
\hline $\mathrm{C}$ & 8.3898000000 & 1.0111000000 & 0.8127000000 \\
\hline $\mathrm{C}$ & 7.0090000000 & 0.8418000000 & 0.8600000000 \\
\hline $\mathrm{H}$ & -3.8348000000 & 1.8558000000 & -3.0256000000 \\
\hline $\mathrm{H}$ & -4.3639000000 & 3.4902000000 & -2.6032000000 \\
\hline $\mathrm{H}$ & -4.5737000000 & 2.1633000000 & -1.4409000000 \\
\hline $\mathrm{H}$ & -1.1482000000 & 0.1323000000 & 0.6354000000 \\
\hline $\mathrm{H}$ & -1.2371000000 & -2.1086000000 & 0.7820000000 \\
\hline $\mathrm{H}$ & -2.3758000000 & -3.4199000000 & -1.2466000000 \\
\hline $\mathrm{H}$ & -3.1486000000 & -1.8830000000 & -1.6302000000 \\
\hline $\mathrm{H}$ & -1.4439000000 & 2.4596000000 & -3.4846000000 \\
\hline $\mathrm{H}$ & -0.5179000000 & 3.4400000000 & -2.3332000000 \\
\hline $\mathrm{H}$ & -1.8439000000 & 4.1683000000 & -3.2558000000 \\
\hline $\mathrm{H}$ & -3.3470000000 & 3.8752000000 & 0.1628000000 \\
\hline $\mathrm{H}$ & -2.9844000000 & 4.9853000000 & -1.1702000000 \\
\hline $\mathrm{H}$ & -1.6690000000 & 4.3307000000 & -0.1811000000 \\
\hline $\mathrm{H}$ & -3.2654000000 & -3.3419000000 & 1.0995000000 \\
\hline $\mathrm{H}$ & -4.5092000000 & -3.4130000000 & -0.1579000000 \\
\hline $\mathrm{H}$ & -5.5670000000 & 1.0396000000 & -0.5863000000 \\
\hline $\mathrm{H}$ & -5.2135000000 & 0.9184000000 & 1.1675000000 \\
\hline $\mathrm{H}$ & -6.8937000000 & 0.0889000000 & 2.7503000000 \\
\hline $\mathrm{H}$ & -9.3122000000 & -0.2468000000 & 3.2245000000 \\
\hline $\mathrm{H}$ & -10.9486000000 & -0.3398000000 & 1.3545000000 \\
\hline $\mathrm{H}$ & -10.1668000000 & -0.0958000000 & -0.9930000000 \\
\hline $\mathrm{H}$ & -7.7522000000 & 0.2445000000 & -1.4725000000 \\
\hline $\mathrm{H}$ & 2.3800000000 & -0.2680000000 & -1.6003000000 \\
\hline $\mathrm{H}$ & 2.0093000000 & -1.8107000000 & -2.4560000000 \\
\hline $\mathrm{H}$ & 5.9934000000 & -1.5557000000 & 1.1180000000 \\
\hline $\mathrm{H}$ & 5.7046000000 & -3.9875000000 & 1.5349000000 \\
\hline $\mathrm{H}$ & 3.7592000000 & -5.1925000000 & 0.5488000000 \\
\hline $\mathrm{H}$ & 2.1116000000 & -3.9594000000 & -0.8362000000 \\
\hline $\mathrm{H}$ & 3.3121000000 & 2.9625000000 & -0.0429000000 \\
\hline $\mathrm{H}$ & 2.4092000000 & 4.2239000000 & 1.9103000000 \\
\hline $\mathrm{H}$ & 2.4477000000 & 3.1950000000 & 4.1747000000 \\
\hline
\end{tabular}




$\begin{array}{cccc}\mathrm{H} & 3.3881000000 & 0.9137000000 & 4.4867000000 \\ \mathrm{H} & 4.2902000000 & -0.3397000000 & 2.5321000000 \\ \mathrm{H} & 6.3869000000 & 0.4001000000 & -2.4504000000 \\ \mathrm{H} & 8.8611000000 & 0.6927000000 & -2.5422000000 \\ \mathrm{H} & 10.1410000000 & 1.0886000000 & -0.4463000000 \\ \mathrm{H} & 8.9514000000 & 1.1876000000 & 1.7358000000 \\ \mathrm{H} & 6.4791000000 & 0.8881000000 & 1.8214000000\end{array}$

Compound ID : $\mathbf{4 d 5 b}$

Calculation : OPT + FREQ, Basis Set : TZVP, Functional : BP86

Imaginary Frequency $=0$

Dipole Moment $=7.82$ debye

$\mathrm{EE}=-0.244916$ hatree

$\begin{array}{lrrr}\mathrm{C} & 0.7413000000 & 7.1430000000 & 3.6462000000 \\ \mathrm{C} & 1.2347000000 & 6.2907000000 & 2.5342000000 \\ \mathrm{C} & 0.6626000000 & 5.0394000000 & 2.2994000000 \\ \mathrm{C} & 1.1331000000 & 4.2323000000 & 1.2733000000 \\ \mathrm{C} & 2.1860000000 & 4.6750000000 & 0.4601000000 \\ \mathrm{~N} & 2.6065000000 & 3.8600000000 & -0.6549000000 \\ \mathrm{C} & 3.9585000000 & 3.4227000000 & -0.8067000000 \\ \mathrm{O} & 4.8784000000 & 4.0608000000 & -0.3149000000 \\ \mathrm{C} & 4.1828000000 & 2.1784000000 & -1.6735000000 \\ \mathrm{~N} & 3.4160000000 & 1.0849000000 & -1.1121000000 \\ \mathrm{P} & 4.0348000000 & -0.4250000000 & -0.9892000000 \\ \mathrm{C} & 3.7361000000 & -1.4617000000 & -2.4485000000 \\ \mathrm{C} & 3.6252000000 & -2.8462000000 & -2.3537000000 \\ \mathrm{C} & 3.3851000000 & -3.6186000000 & -3.4851000000 \\ \mathrm{C} & 3.2524000000 & -3.0055000000 & -4.7272000000 \\ \mathrm{C} & 3.3592000000 & -1.6228000000 & -4.8310000000 \\ \mathrm{C} & 3.5994000000 & -0.8563000000 & -3.6940000000 \\ \mathrm{C} & 5.8251000000 & -0.5363000000 & -0.6772000000 \\ \mathrm{C} & 6.6190000000 & -1.5206000000 & -1.2597000000 \\ \mathrm{C} & 7.9878000000 & -1.5658000000 & -1.0188000000 \\ \mathrm{C} & 8.5768000000 & -0.6191000000 & -0.1860000000 \\ \mathrm{C} & 7.7924000000 & 0.3669000000 & 0.4025000000 \\ \mathrm{C} & 6.4224000000 & 0.4054000000 & 0.1573000000 \\ \mathrm{C} & 3.1966000000 & -1.3010000000 & 0.3821000000 \\ \mathrm{C} & 1.8630000000 & -1.1240000000 & 0.7756000000 \\ \mathrm{C} & 0.9412000000 & -0.1896000000 & 0.0348000000 \\ \mathrm{O} & -0.4429000000 & -0.3705000000 & 0.3114000000 \\ \mathrm{C} & -1.1309000000 & -1.3978000000 & -0.2687000000 \\ \mathrm{O} & -0.5817000000 & -2.1260000000 & -1.0684000000 \\ \mathrm{C} & -2.6039000000 & -1.4932000000 & 0.1595000000 \\ \mathrm{C} & -3.4442000000 & -0.6913000000 & -0.8472000000 \\ \mathrm{C} & -4.9179000000 & -1.0555000000 & -0.7598000000 \\ & & & \end{array}$




\begin{tabular}{|c|c|c|c|}
\hline $\mathrm{C}$ & -5.7541000000 & -0.2757000000 & -1.7504000000 \\
\hline $\mathrm{O}$ & -5.4654000000 & 0.7478000000 & -2.3374000000 \\
\hline $\mathrm{O}$ & -6.9691000000 & -0.8658000000 & -1.9664000000 \\
\hline $\mathrm{C}$ & -7.9368000000 & -0.2296000000 & -2.7942000000 \\
\hline $\mathrm{C}$ & -8.7715000000 & 0.7581000000 & -2.0311000000 \\
\hline $\mathrm{C}$ & -9.7888000000 & 0.3077000000 & -1.1890000000 \\
\hline $\mathrm{C}$ & -10.5744000000 & 1.2213000000 & -0.4955000000 \\
\hline $\mathrm{C}$ & -10.3494000000 & 2.5864000000 & -0.6410000000 \\
\hline $\mathrm{C}$ & -9.3361000000 & 3.0377000000 & -1.4800000000 \\
\hline $\mathrm{C}$ & -8.5468000000 & 2.1273000000 & -2.1746000000 \\
\hline $\mathrm{N}$ & -2.8844000000 & -1.0185000000 & 1.5385000000 \\
\hline $\mathrm{C}$ & -2.4695000000 & -1.7568000000 & 2.6895000000 \\
\hline $\mathrm{O}$ & -2.4305000000 & -1.2443000000 & 3.7965000000 \\
\hline $\mathrm{O}$ & -2.2007000000 & -3.0607000000 & 2.4181000000 \\
\hline $\mathrm{C}$ & -2.0579000000 & -4.0441000000 & 3.4766000000 \\
\hline $\mathrm{C}$ & -0.8487000000 & -3.7680000000 & 4.3715000000 \\
\hline $\mathrm{C}$ & -1.8282000000 & -5.3418000000 & 2.6868000000 \\
\hline $\mathrm{C}$ & -3.3342000000 & -4.1648000000 & 4.3106000000 \\
\hline $\mathrm{C}$ & 1.3806000000 & -1.8225000000 & 1.8872000000 \\
\hline $\mathrm{C}$ & 2.1821000000 & -2.7219000000 & 2.5759000000 \\
\hline $\mathrm{C}$ & 3.4966000000 & -2.9204000000 & 2.1729000000 \\
\hline $\mathrm{C}$ & 3.9892000000 & -2.2048000000 & 1.0889000000 \\
\hline $\mathrm{C}$ & 2.7584000000 & 5.9312000000 & 0.6884000000 \\
\hline $\mathrm{C}$ & 2.2829000000 & 6.7269000000 & 1.7232000000 \\
\hline $\mathrm{H}$ & 1.2930000000 & 6.9240000000 & 4.5708000000 \\
\hline $\mathrm{H}$ & -0.3243000000 & 6.9724000000 & 3.8494000000 \\
\hline $\mathrm{H}$ & 0.8709000000 & 8.2116000000 & 3.4297000000 \\
\hline $\mathrm{H}$ & -0.1620000000 & 4.6909000000 & 2.9312000000 \\
\hline $\mathrm{H}$ & 0.6733000000 & 3.2487000000 & 1.1086000000 \\
\hline $\mathrm{H}$ & 1.9443000000 & 3.1373000000 & -0.8486000000 \\
\hline $\mathrm{H}$ & 5.2711000000 & 1.9621000000 & -1.7476000000 \\
\hline $\mathrm{H}$ & 3.8299000000 & 2.3887000000 & -2.7065000000 \\
\hline $\mathrm{H}$ & 3.7143000000 & -3.3286000000 & -1.3706000000 \\
\hline $\mathrm{H}$ & 3.2929000000 & -4.7061000000 & -3.3967000000 \\
\hline $\mathrm{H}$ & 3.0593000000 & -3.6101000000 & -5.6196000000 \\
\hline $\mathrm{H}$ & 3.2497000000 & -1.1358000000 & -5.8057000000 \\
\hline $\mathrm{H}$ & 3.6714000000 & 0.2364000000 & -3.7827000000 \\
\hline $\mathrm{H}$ & 6.1555000000 & -2.2667000000 & -1.9205000000 \\
\hline $\mathrm{H}$ & 8.6007000000 & -2.3437000000 & -1.4857000000 \\
\hline $\mathrm{H}$ & 9.6550000000 & -0.6497000000 & 0.0034000000 \\
\hline $\mathrm{H}$ & 8.2507000000 & 1.1180000000 & 1.0551000000 \\
\hline $\mathrm{H}$ & 5.8148000000 & 1.1940000000 & 0.6223000000 \\
\hline $\mathrm{H}$ & 1.1274000000 & -0.2257000000 & -1.0569000000 \\
\hline $\mathrm{H}$ & 1.0727000000 & 0.8545000000 & 0.3845000000 \\
\hline $\mathrm{H}$ & -2.8988000000 & -2.5735000000 & 0.1063000000 \\
\hline $\mathrm{H}$ & -3.0728000000 & -0.8971000000 & -1.8721000000 \\
\hline
\end{tabular}




$\begin{array}{cccc}\mathrm{H} & -3.3077000000 & 0.4005000000 & -0.6980000000 \\ \mathrm{H} & -5.0547000000 & -2.1446000000 & -0.9236000000 \\ \mathrm{H} & -5.3157000000 & -0.8505000000 & 0.2554000000 \\ \mathrm{H} & -8.5356000000 & -1.1011000000 & -3.1157000000 \\ \mathrm{H} & -7.4784000000 & 0.2300000000 & -3.6909000000 \\ \mathrm{H} & -9.9667000000 & -0.7676000000 & -1.0749000000 \\ \mathrm{H} & -11.3712000000 & 0.8650000000 & 0.1653000000 \\ \mathrm{H} & -10.9693000000 & 3.3048000000 & -0.0949000000 \\ \mathrm{H} & -9.1566000000 & 4.1119000000 & -1.5938000000 \\ \mathrm{H} & -7.7419000000 & 2.4832000000 & -2.8295000000 \\ \mathrm{H} & -2.7639000000 & -0.0320000000 & 1.6457000000 \\ \mathrm{H} & 0.0727000000 & -3.5977000000 & 3.7871000000 \\ \mathrm{H} & -0.6648000000 & -4.6277000000 & 5.0295000000 \\ \mathrm{H} & -0.9992000000 & -2.8909000000 & 5.0151000000 \\ \mathrm{H} & -0.9207000000 & -5.2891000000 & 2.0705000000 \\ \mathrm{H} & -2.6702000000 & -5.5689000000 & 2.0193000000 \\ \mathrm{H} & -1.7135000000 & -6.1899000000 & 3.3744000000 \\ \mathrm{H} & -3.5109000000 & -3.2741000000 & 4.9283000000 \\ \mathrm{H} & -3.2630000000 & -5.0250000000 & 4.9892000000 \\ \mathrm{H} & -4.2190000000 & -4.3145000000 & 3.6779000000 \\ \mathrm{H} & 0.3493000000 & -1.6725000000 & 2.2340000000 \\ \mathrm{H} & 1.7560000000 & -3.2736000000 & 3.4284000000 \\ \mathrm{H} & 4.1394000000 & -3.6285000000 & 2.7056000000 \\ \mathrm{H} & 5.0352000000 & -2.3544000000 & 0.7835000000 \\ \mathrm{H} & 3.5885000000 & 6.2882000000 & 0.0671000000 \\ \mathrm{H} & 2.7353000000 & 7.7089000000 & 1.9021000000\end{array}$

Compound ID : A1-7m-TS

Calculation : OPT + FREQ, Basis Set : TZVP, Functional : BP86 Imaginary Frequency $=1(\mathrm{TS})$

Dipole Moment $=9.2$ debye

$\mathrm{E}=-0.242899$ hatree

$\begin{array}{lrrr}\mathrm{C} & -5.7823000000 & -4.0719000000 & -0.8383000000 \\ \mathrm{C} & -4.2454000000 & -4.0719000000 & -0.8383000000 \\ \mathrm{O} & -3.9303000000 & -2.6587000000 & -0.8383000000 \\ \mathrm{C} & -2.6480000000 & -2.2087000000 & -0.9376000000 \\ \mathrm{~N} & -2.7081000000 & -0.8159000000 & -1.0835000000 \\ \mathrm{O} & -1.6333000000 & -2.8918000000 & -0.9178000000 \\ \mathrm{C} & -1.5034000000 & 0.0446000000 & -1.1472000000 \\ \mathrm{C} & -1.6161000000 & 1.1769000000 & -0.1144000000 \\ \mathrm{O} & -1.5941000000 & 2.3715000000 & -0.3235000000 \\ \mathrm{C} & -1.3538000000 & 0.6267000000 & -2.5622000000 \\ \mathrm{C} & -3.7319000000 & -4.7630000000 & 0.4255000000 \\ \mathrm{C} & -3.7339000000 & -4.7781000000 & -2.0936000000 \\ \mathrm{C} & -0.7806000000 & -0.4021000000 & -3.5278000000\end{array}$




\begin{tabular}{|c|c|c|c|}
\hline $\mathrm{C}$ & -0.8736000000 & 0.1110000000 & -4.9490000000 \\
\hline $\mathrm{O}$ & -0.0326000000 & 0.7153000000 & -5.5799000000 \\
\hline $\mathrm{O}$ & -2.0684000000 & -0.2035000000 & -5.5419000000 \\
\hline $\mathrm{C}$ & -2.3682000000 & 0.2870000000 & -6.8427000000 \\
\hline $\mathrm{C}$ & -2.9836000000 & 1.6569000000 & -6.8026000000 \\
\hline $\mathrm{C}$ & -2.2846000000 & 2.7556000000 & -7.3021000000 \\
\hline $\mathrm{C}$ & -2.8692000000 & 4.0176000000 & -7.2851000000 \\
\hline $\mathrm{C}$ & -4.1488000000 & 4.1882000000 & -6.7684000000 \\
\hline $\mathrm{C}$ & -4.8468000000 & 3.0943000000 & -6.2669000000 \\
\hline $\mathrm{C}$ & -4.2676000000 & 1.8309000000 & -6.2847000000 \\
\hline $\mathrm{O}$ & -1.7956000000 & 0.7235000000 & 1.1691000000 \\
\hline $\mathrm{C}$ & -1.8730000000 & 1.6923000000 & 2.2101000000 \\
\hline $\mathrm{C}$ & -1.4421000000 & 1.1876000000 & 3.5690000000 \\
\hline $\mathrm{C}$ & -0.4095000000 & 0.3183000000 & 3.9324000000 \\
\hline $\mathrm{C}$ & -0.2325000000 & 0.0264000000 & 5.2921000000 \\
\hline $\mathrm{C}$ & -1.0084000000 & 0.5971000000 & 6.2856000000 \\
\hline $\mathrm{C}$ & -2.0068000000 & 1.4981000000 & 5.9267000000 \\
\hline $\mathrm{C}$ & -2.2141000000 & 1.7772000000 & 4.5895000000 \\
\hline $\mathrm{N}$ & 1.1810000000 & 0.2204000000 & 1.5335000000 \\
\hline $\mathrm{C}$ & 1.8231000000 & -0.4950000000 & 0.4486000000 \\
\hline $\mathrm{C}$ & 2.7989000000 & 0.4247000000 & -0.2959000000 \\
\hline $\mathrm{N}$ & 3.7957000000 & 1.0650000000 & 0.4825000000 \\
\hline $\mathrm{O}$ & 2.7651000000 & 0.5542000000 & -1.5118000000 \\
\hline $\mathrm{C}$ & 4.7293000000 & 2.0718000000 & 0.0792000000 \\
\hline $\mathrm{C}$ & 5.3276000000 & 2.8357000000 & 1.0980000000 \\
\hline $\mathrm{C}$ & 6.2851000000 & 3.7871000000 & 0.7863000000 \\
\hline $\mathrm{C}$ & 6.6656000000 & 4.0024000000 & -0.5399000000 \\
\hline $\mathrm{C}$ & 6.0725000000 & 3.2444000000 & -1.5475000000 \\
\hline $\mathrm{C}$ & 5.1132000000 & 2.2837000000 & -1.2496000000 \\
\hline $\mathrm{C}$ & 7.6806000000 & 5.0378000000 & -0.8610000000 \\
\hline$P$ & 0.8327000000 & -0.5776000000 & 2.9243000000 \\
\hline $\mathrm{C}$ & 0.2322000000 & -2.2931000000 & 2.8186000000 \\
\hline $\mathrm{C}$ & 0.9345000000 & -3.2165000000 & 2.0476000000 \\
\hline $\mathrm{C}$ & 0.4849000000 & -4.5263000000 & 1.9185000000 \\
\hline $\mathrm{C}$ & -0.6820000000 & -4.9249000000 & 2.5626000000 \\
\hline $\mathrm{C}$ & -1.3906000000 & -4.0113000000 & 3.3358000000 \\
\hline $\mathrm{C}$ & -0.9314000000 & -2.7040000000 & 3.4609000000 \\
\hline $\mathrm{C}$ & 2.3366000000 & -0.6701000000 & 3.9689000000 \\
\hline $\mathrm{C}$ & 2.7705000000 & -1.8411000000 & 4.5813000000 \\
\hline $\mathrm{C}$ & 3.9578000000 & -1.8658000000 & 5.3057000000 \\
\hline $\mathrm{C}$ & 4.7252000000 & -0.7116000000 & 5.4213000000 \\
\hline $\mathrm{C}$ & 4.3004000000 & 0.4642000000 & 4.8116000000 \\
\hline $\mathrm{C}$ & 3.1101000000 & 0.4815000000 & 4.0912000000 \\
\hline $\mathrm{H}$ & -6.1631000000 & -5.1016000000 & -0.8405000000 \\
\hline $\mathrm{H}$ & -6.1905000000 & -3.5657000000 & -1.7231000000 \\
\hline $\mathrm{H}$ & -6.1906000000 & -3.5698000000 & 0.0488000000 \\
\hline
\end{tabular}




\begin{tabular}{|c|c|c|c|}
\hline & -3.5871000000 & -0.3453000000 & \\
\hline & -0.5735000000 & -0.5520000000 & -0.9024000000 \\
\hline & -2.3276000000 & 1.0106000000 & -2.9298000000 \\
\hline & -0.6786000000 & 1.5077000000 & -2.5293000000 \\
\hline & -4.0360000000 & -4.2267000000 & 1.3350000000 \\
\hline & -2.6344000000 & -4.8403000000 & 0.4452000000 \\
\hline & -4.1347000000 & -5.7821000000 & 0.4914000000 \\
\hline & -4.1354000000 & -5.7989000000 & -2.1429000000 \\
\hline & -4.0450000000 & -4.2547000000 & -3.0071000000 \\
\hline & -2.6388000000 & -4.8565000000 & 5000000 \\
\hline & 2000000 & 1000000 & 3000000 \\
\hline & 6000000 & -1.3716000000 & 3000000 \\
\hline & 4000000 & & \\
\hline & 6000000 & 81000000 & 891000000 \\
\hline & -1.2 & & 00000 \\
\hline & -2.31 & 4.8 & 00000 \\
\hline & -4.60 & 5.18 & 0000 \\
\hline & -5.8 & 3.2 & 0000 \\
\hline & 000000 & 7000000 & 000000 \\
\hline & -2.9 & & 000000 \\
\hline & -1.2 & 2.6 & 0000 \\
\hline & 0.5 & -0.6 & 0000 \\
\hline & -0.8 & & 0000 \\
\hline & -2.6 & & 0000 \\
\hline & -3.0 & & 000 \\
\hline & 2.37 & -1.41 & 0000 \\
\hline & 1.0 & -0.8 & 0000 \\
\hline & 3.64 & 1.02 & 0000 \\
\hline $\mathrm{H}$ & 5.0 & 2.6 & 0000 \\
\hline & 6.72 & 4.3 & 0000 \\
\hline & 6.36 & 3.40 & 00000 \\
\hline & 4.66 & 1.70 & 00000 \\
\hline $\mathrm{H}$ & 8.02 & 4.97 & 00000 \\
\hline $\mathrm{H}$ & 7.26 & 6.0 & 00000 \\
\hline $\mathrm{H}$ & 8.56 & 4.94 & 00000 \\
\hline $\mathrm{H}$ & 1.849 & -2.9010000000 & 1.5198000000 \\
\hline $\mathrm{H}$ & 1.04 & 77000000 & 00000 \\
\hline $\mathrm{H}$ & -1.0465000000 & -5.9520000000 & 2.4530000000 \\
\hline $\mathrm{H}$ & -2.31 & 58000000 & 5000000 \\
\hline $\mathrm{H}$ & 6000000 & -1.9850000000 & 4.0680000000 \\
\hline $\mathrm{H}$ & 2.1713000000 & -2.7565000000 & 4.4827000000 \\
\hline & 4.2887000000 & -2.7943000000 & 5.7825000000 \\
\hline $\mathrm{H}$ & 5.6618000000 & -0.7293000000 & 5.9885000000 \\
\hline & 4.9024000000 & 1.3760000000 & 4.8907000000 \\
\hline & 2.7787000000 & 1.4156000000 & 3.6165000000 \\
\hline
\end{tabular}


Compound ID : B1-11m-TS

Calculation : OPT + FREQ, Basis Set : TZVP, Functional : BP86

Imaginary Frequency $=1(\mathrm{TS})$

Dipole Moment $=7.25$ debye

$\mathrm{E}=-0.243578$ hatree

$\begin{array}{lrrr}\mathrm{C} & -6.7244000000 & 0.1718000000 & -1.5291000000 \\ \mathrm{C} & -6.4779000000 & -1.2652000000 & -1.9979000000 \\ \mathrm{O} & -5.5900000000 & -1.3243000000 & -3.133900000 \\ \mathrm{C} & -4.2743000000 & -0.9970000000 & -3.1089000000 \\ \mathrm{~N} & -3.5802000000 & -0.4214000000 & -1.9908000000 \\ \mathrm{O} & -3.7903000000 & -1.1843000000 & -4.2119000000 \\ \mathrm{C} & -2.0908000000 & -0.3898000000 & -2.0496000000 \\ \mathrm{C} & -1.4675000000 & -1.4832000000 & -1.1750000000 \\ \mathrm{O} & -2.0660000000 & -2.3305000000 & -0.5369000000 \\ \mathrm{C} & -1.5755000000 & 1.0144000000 & -1.6997000000 \\ \mathrm{C} & -6.0078000000 & -2.1522000000 & -0.8421000000 \\ \mathrm{C} & -7.7834000000 & -1.8394000000 & -2.5744000000 \\ \mathrm{~N} & 3.1083000000 & -0.0837000000 & -1.1240000000 \\ \mathrm{C} & 3.9618000000 & 0.7991000000 & -1.8808000000 \\ \mathrm{C} & 3.7614000000 & 2.2357000000 & -1.3757000000 \\ \mathrm{~N} & 2.6313000000 & 2.9396000000 & -1.8726000000 \\ \mathrm{O} & 4.5420000000 & 2.7724000000 & -0.5942000000 \\ \mathrm{C} & 2.1355000000 & 4.1447000000 & -1.2481000000 \\ \mathrm{C} & 0.8061000000 & 4.2023000000 & -0.8107000000 \\ \mathrm{C} & 0.2985000000 & 5.3838000000 & -0.2883000000 \\ \mathrm{C} & 1.1067000000 & 6.5171000000 & -0.1872000000 \\ \mathrm{C} & 2.4335000000 & 6.4520000000 & -0.6147000000 \\ \mathrm{C} & 2.9502000000 & 5.2777000000 & -1.1473000000 \\ \mathrm{C} & -1.6398000000 & 1.4092000000 & -0.2324000000 \\ \mathrm{C} & -3.0206000000 & 1.8585000000 & 0.1886000000 \\ \mathrm{O} & -3.5542000000 & 1.0632000000 & 1.1601000000 \\ \mathrm{O} & -3.6549000000 & 2.8111000000 & -0.2195000000 \\ \mathrm{C} & -4.8940000000 & 1.3157000000 & 1.5914000000 \\ \mathrm{C} & -5.1589000000 & 0.3651000000 & 2.7214000000 \\ \mathrm{C} & -5.2331000000 & -1.0070000000 & 2.4787000000 \\ \mathrm{C} & -5.4888000000 & -1.8862000000 & 3.5244000000 \\ \mathrm{C} & -5.6760000000 & -1.3998000000 & 4.8143000000 \\ \mathrm{C} & -5.6041000000 & -0.0325000000 & 5.0583000000 \\ \mathrm{C} & -5.3428000000 & 0.8505000000 & 4.0161000000 \\ \mathrm{C} & 0.5553000000 & 7.7836000000 & 0.3588000000 \\ \mathrm{O} & -0.1099000000 & -1.4711000000 & -1.2101000000 \\ \mathrm{C} & 0.6558000000 & -2.4249000000 & -0.4827000000 \\ \mathrm{C} & 1.0466000000 & -1.9369000000 & 0.8909000000 \\ \mathrm{C} & 0.0891000000 & -2.0367000000 & 1.9062000000\end{array}$




\begin{tabular}{|c|c|c|c|}
\hline & 0.3767000000 & -1.6270000000 & \\
\hline & 1.6365000000 & -1.1199000000 & 3.4967000000 \\
\hline & 2.5921000000 & -1.0324000000 & 2.4920000000 \\
\hline & 2.3208000000 & -1.4369000000 & 1.1859000000 \\
\hline & 3.6733000000 & -1.1591000000 & -0.0226000000 \\
\hline & 5.1803000000 & -0.7698000000 & 1000000 \\
\hline & 5.6651000000 & 0.5336000000 & 3000000 \\
\hline 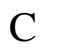 & 6.8187000000 & 0.8084000000 & 000000 \\
\hline $\mathrm{C}$ & 7.4981000000 & -0.2126000000 & 2.389 \\
\hline & 7.0230000000 & 34000000 & 2.306 \\
\hline E & 5.8721000000 & 0000000 & 1.576 \\
\hline & 4.0357000000 & $-2.7 \mathrm{C}$ & -0.75 \\
\hline $\mathrm{C}$ & 4.56590000 & -2.8 & \\
\hline $\mathrm{C}$ & 4.87820000 & -4.0 & -2.63 \\
\hline $\mathrm{C}$ & 4.6616000000 & -5.2 & -1.93 \\
\hline $\mathrm{C}$ & 4.12940 & -5.2 & \\
\hline C & 3.818 & -3.9 & -0.0 \\
\hline $\mathrm{H}$ & -7.66 & & \\
\hline $\mathrm{H}$ & -6.80 & 0.8 & -2.3 \\
\hline $\mathrm{H}$ & -5.93 & & \\
\hline $\mathrm{H}$ & -3.94530 & -0.7 & \\
\hline I & -1.76840 & & \\
\hline $\mathrm{H}$ & -0.51240 & & -2.0 \\
\hline $\mathrm{H}$ & -2.11490 & 33000000 & -2.3 \\
\hline $\mathrm{H}$ & -5.666 & -3.1 & -1.1 \\
\hline & -6.83070 & -2.3 & \\
\hline $\mathrm{H}$ & -5.1816 & -1.6 & -0.2 \\
\hline I & -8.5678 & -1.8 & \\
\hline $\mathrm{H}$ & & & \\
\hline $\mathrm{H}$ & -7.64730 & -2.8651000000 & \\
\hline $\mathrm{H}$ & 5.041100 & 0.5363000000 & -1.84 \\
\hline $\mathrm{H}$ & 3.6495 & 0.7 & -2.9 \\
\hline $\mathrm{H}$ & 1.89490000 & 2.33 & -2.10 \\
\hline $\mathrm{H}$ & 0.14970 & 3.3 & -0.8 \\
\hline I & -0.7443000000 & 52000000 & \\
\hline $\mathrm{H}$ & 3.0749000000 & 8000000 & -0.53 \\
\hline H & 3.9971000000 & 5.2431000000 & -1.47 \\
\hline & -1.2677000000 & 0.58 & \\
\hline $\mathrm{H}$ & -0.9421000000 & 2.2633000000 & -0.0624000000 \\
\hline $\mathrm{H}$ & -5.0116000000 & 2.3712000000 & 1.9037000000 \\
\hline $\mathrm{H}$ & -5.5833000000 & 1.1272000000 & 0.7368000000 \\
\hline & -5.0866000000 & -1.38 & \\
\hline $\mathrm{H}$ & -5.5428000000 & -2.9625000000 & 3.330600000 \\
\hline & & -2.0931000000 & \\
\hline $\mathrm{H}$ & -5.7513000000 & 0.3510000000 & 6.073400000 \\
\hline & -5.2813000000 & 1.9271000000 & 4.213300000 \\
\hline
\end{tabular}




$\begin{array}{rrrr}\mathrm{H} & -0.2417000000 & 7.5996000000 & 1.0914000000 \\ \mathrm{H} & 1.3289000000 & 8.3855000000 & 0.8538000000 \\ \mathrm{H} & 0.1275000000 & 8.3941000000 & -0.4483000000 \\ \mathrm{H} & 0.1516000000 & -3.4087000000 & -0.4141000000 \\ \mathrm{H} & 1.5380000000 & -2.5259000000 & -1.1450000000 \\ \mathrm{H} & -0.9037000000 & -2.4473000000 & 1.6798000000 \\ \mathrm{H} & -0.3864000000 & -1.7034000000 & 3.9832000000 \\ \mathrm{H} & 1.8756000000 & -0.7901000000 & 4.5131000000 \\ \mathrm{H} & 3.5863000000 & -0.6312000000 & 2.7376000000 \\ \mathrm{H} & 5.1641000000 & 1.3838000000 & 0.4777000000 \\ \mathrm{H} & 7.1889000000 & 1.8382000000 & 1.7921000000 \\ \mathrm{H} & 8.4044000000 & 0.0065000000 & 2.9636000000 \\ \mathrm{H} & 7.5547000000 & -2.3303000000 & 2.8133000000 \\ \mathrm{H} & 5.5055000000 & -2.8215000000 & 1.5078000000 \\ \mathrm{H} & 4.7322000000 & -1.9061000000 & -2.5945000000 \\ \mathrm{H} & 5.2927000000 & -4.0846000000 & -3.6457000000 \\ \mathrm{H} & 4.9069000000 & -6.2057000000 & -2.4021000000 \\ \mathrm{H} & 3.9539000000 & -6.1375000000 & -0.1039000000 \\ \mathrm{H} & 3.3871000000 & -3.9578000000 & 0.9422000000\end{array}$

Compound ID : A2-7m

Calculation : OPT + FREQ, Basis Set : TZVP, Functional : BP86

Imaginary Frequency $=0$

Dipole Moment $=9.82$ debye

$\mathrm{EE}=-0.288331$ hatree

$\begin{array}{lrrr}\mathrm{C} & -9.6580000000 & 2.0426000000 & -2.0450000000 \\ \mathrm{C} & -8.1780000000 & 2.0426000000 & -2.0450000000 \\ \mathrm{C} & -7.4863000000 & 3.2559000000 & -2.0450000000 \\ \mathrm{C} & -6.1029000000 & 3.2715000000 & -2.0549000000 \\ \mathrm{C} & -5.3936000000 & 2.0596000000 & -2.0905000000 \\ \mathrm{~N} & -3.9588000000 & 2.1336000000 & -2.1652000000 \\ \mathrm{C} & -3.0328000000 & 1.2214000000 & -1.6034000000 \\ \mathrm{O} & -3.4230000000 & 0.3250000000 & -0.8729000000 \\ \mathrm{C} & -1.5719000000 & 1.4828000000 & -1.9795000000 \\ \mathrm{~N} & -0.6159000000 & 0.8070000000 & -1.0528000000 \\ \mathrm{C} & -0.3743000000 & -0.6198000000 & -1.3928000000 \\ \mathrm{O} & -0.5752000000 & -0.9663000000 & -2.5332000000 \\ \mathrm{C} & -0.0351000000 & -1.6058000000 & -0.2559000000 \\ \mathrm{C} & 1.4352000000 & -2.0540000000 & -0.3693000000 \\ \mathrm{C} & 1.9246000000 & -2.6302000000 & 0.9483000000 \\ \mathrm{C} & 3.4213000000 & -2.8246000000 & 1.0093000000 \\ \mathrm{O} & 4.2479000000 & -2.7493000000 & 0.1278000000 \\ \mathrm{O} & 3.8184000000 & -3.1625000000 & 2.2824000000 \\ \mathrm{C} & 5.1573000000 & -3.5521000000 & 2.5380000000 \\ \mathrm{C} & 6.1584000000 & -2.4373000000 & 2.6112000000\end{array}$




\begin{tabular}{|c|c|c|c|}
\hline $\mathrm{C}$ & 7.3839000000 & -2.5702000000 & 1.9544000000 \\
\hline $\mathrm{C}$ & 8.3564000000 & -1.5844000000 & 2.0867000000 \\
\hline $\mathrm{C}$ & 8.1162000000 & -0.4585000000 & 2.8690000000 \\
\hline $\mathrm{C}$ & 6.8937000000 & -0.3212000000 & 3.5170000000 \\
\hline $\mathrm{C}$ & 5.9165000000 & -1.3008000000 & 3.3831000000 \\
\hline $\mathrm{N}$ & -0.9658000000 & -2.7587000000 & -0.3954000000 \\
\hline $\mathrm{C}$ & -1.4011000000 & -3.4756000000 & 0.7399000000 \\
\hline $\mathrm{O}$ & -1.1693000000 & -3.2483000000 & 1.9214000000 \\
\hline $\mathrm{O}$ & -2.2634000000 & -4.4365000000 & 0.3014000000 \\
\hline $\mathrm{C}$ & -2.9728000000 & -5.2835000000 & 1.2395000000 \\
\hline $\mathrm{C}$ & -1.9924000000 & -6.2040000000 & 1.9886000000 \\
\hline $\mathrm{C}$ & -3.8360000000 & -4.4778000000 & 2.2229000000 \\
\hline $\mathrm{C}$ & -3.8857000000 & -6.1131000000 & 0.3232000000 \\
\hline $\mathrm{P}$ & 0.4209000000 & 2.0350000000 & -0.2606000000 \\
\hline $\mathrm{C}$ & -0.8867000000 & 2.9603000000 & 0.5986000000 \\
\hline $\mathrm{C}$ & -1.4938000000 & 2.3568000000 & 1.6995000000 \\
\hline $\mathrm{C}$ & -2.4946000000 & 2.9997000000 & 2.4174000000 \\
\hline $\mathrm{C}$ & -2.9291000000 & 4.2631000000 & 2.0311000000 \\
\hline $\mathrm{C}$ & -2.3701000000 & 4.8579000000 & 0.9062000000 \\
\hline $\mathrm{C}$ & -1.3674000000 & 4.2058000000 & 0.1958000000 \\
\hline $\mathrm{C}$ & 1.4793000000 & 1.4083000000 & 1.0149000000 \\
\hline $\mathrm{C}$ & 2.8000000000 & 1.8630000000 & 1.0304000000 \\
\hline $\mathrm{C}$ & 3.6736000000 & 1.4809000000 & 2.0447000000 \\
\hline $\mathrm{C}$ & 3.2344000000 & 0.6345000000 & 3.0589000000 \\
\hline $\mathrm{C}$ & 1.9264000000 & 0.1576000000 & 3.0447000000 \\
\hline $\mathrm{C}$ & 1.0638000000 & 0.5334000000 & 2.0208000000 \\
\hline $\mathrm{C}$ & 1.3224000000 & 2.9592000000 & -1.4336000000 \\
\hline $\mathrm{C}$ & 2.1236000000 & 2.2859000000 & -2.3616000000 \\
\hline $\mathrm{C}$ & 2.6123000000 & 0.7984000000 & -2.1964000000 \\
\hline $\mathrm{O}$ & 2.5831000000 & 0.0809000000 & -3.2557000000 \\
\hline $\mathrm{C}$ & 2.6408000000 & 2.9913000000 & -3.4665000000 \\
\hline $\mathrm{C}$ & 2.4889000000 & 4.3570000000 & -3.5660000000 \\
\hline $\mathrm{C}$ & 1.8385000000 & 5.0579000000 & -2.5375000000 \\
\hline $\mathrm{C}$ & 1.2590000000 & 4.3626000000 & -1.4995000000 \\
\hline $\mathrm{C}$ & -6.0846000000 & 0.8441000000 & -2.0821000000 \\
\hline $\mathrm{C}$ & -7.4715000000 & 0.8433000000 & -2.0479000000 \\
\hline $\mathrm{H}$ & -10.0428000000 & 2.2973000000 & -3.0391000000 \\
\hline $\mathrm{H}$ & -10.0667000000 & 1.0634000000 & -1.7629000000 \\
\hline $\mathrm{H}$ & -10.0403000000 & 2.7846000000 & -1.3332000000 \\
\hline $\mathrm{H}$ & -8.0433000000 & 4.1984000000 & -2.0213000000 \\
\hline $\mathrm{H}$ & -5.5771000000 & 4.2320000000 & -2.0462000000 \\
\hline $\mathrm{H}$ & -3.6117000000 & 2.8296000000 & -2.7866000000 \\
\hline $\mathrm{H}$ & -1.4144000000 & 1.1424000000 & -3.0303000000 \\
\hline $\mathrm{H}$ & -1.3557000000 & 2.5755000000 & -1.9725000000 \\
\hline $\mathrm{H}$ & -0.2315000000 & -1.1276000000 & 0.7443000000 \\
\hline $\mathrm{H}$ & 2.0730000000 & -1.1941000000 & -0.6974000000 \\
\hline
\end{tabular}




\begin{tabular}{|c|c|c|c|}
\hline $\mathrm{H}$ & 1.5692000000 & -2.8081000000 & -1.1910000000 \\
\hline & 1.6096000000 & -1.9790000000 & 1.7934000000 \\
\hline & 1.4480000000 & -3.6142000000 & 1.1388000000 \\
\hline & 5.4728000000 & -4.3377000000 & 1.8243000000 \\
\hline & 5.0430000000 & -4.0074000000 & 3.5378000000 \\
\hline & 7.5759000000 & -3.4498000000 & 1.3280000000 \\
\hline & 9.3113000000 & -1.6905000000 & 1.5625000000 \\
\hline & 8.8759000000 & 0.3260000000 & 2.9558000000 \\
\hline & 6.6955000000 & 0.5614000000 & 4.1365000000 \\
\hline & 4.9375000000 & -1.1578000000 & 3.8691000000 \\
\hline$\vec{H}$ & -0.8814000000 & -3.2635000000 & -1.2616000000 \\
\hline $\boldsymbol{H}$ & -0.9415000000 & -5.9406000000 & \\
\hline $\mathbf{H}$ & -2.1536000000 & -6.1487000000 & 3.0752000000 \\
\hline $\mathrm{H}$ & -2.1137000000 & -7.2537000000 & \\
\hline $\mathrm{H}$ & -4.8657000000 & -4.8582000000 & 2.2554000000 \\
\hline $\mathrm{H}$ & -3.4361000000 & -4.5368000000 & \\
\hline $\mathrm{H}$ & -3.8991000000 & -3.4136000000 & 1.956400000 \\
\hline $\mathrm{H}$ & -4.6715000000 & -5.4922000000 & \\
\hline $\mathrm{H}$ & -3.3327000000 & -6.5911000000 & -0.4959000000 \\
\hline $\mathrm{H}$ & -4.3761000000 & -6.9092000000 & 0.898300000 \\
\hline $\mathrm{H}$ & -1.1957000000 & 1.3413000000 & 2.0089000000 \\
\hline $\mathrm{H}$ & -2.9470000000 & 2.5011000000 & 3.2834000000 \\
\hline $\mathrm{H}$ & -3.7113000000 & 4.7750000000 & 2.60 \\
\hline $\mathrm{H}$ & -2.7143000000 & 5.8448000000 & 0.5704000000 \\
\hline $\mathrm{H}$ & -0.9629000000 & 4.7008000000 & -0.7004000000 \\
\hline $\mathrm{H}$ & 3.1659000000 & 2.5052000000 & 0.2138000000 \\
\hline $\mathrm{H}$ & 4.7093000000 & 1.8360000000 & \\
\hline $\mathrm{H}$ & 3.9275000000 & 0.3173000000 & 3.8563000000 \\
\hline $\mathrm{H}$ & 1.5813000000 & -0.5285000000 & 3.8280000000 \\
\hline $\mathrm{H}$ & 0.0379000000 & 0.1113000000 & 1.9982000000 \\
\hline $\mathrm{H}$ & 1.9688000000 & 0.4048000000 & -1.3232000000 \\
\hline $\mathrm{H}$ & 3.6182000000 & 0.9465000000 & -1.7213000000 \\
\hline $\mathrm{H}$ & 3.1752000000 & 2.4085000000 & -4.2335000000 \\
\hline $\mathrm{H}$ & 2.8908000000 & 4.9034000000 & -4.4251000000 \\
\hline $\mathrm{H}$ & 1.7925000000 & 6.1512000000 & -2.5669000000 \\
\hline $\mathrm{H}$ & 0.7592000000 & 4.9269000000 & -0.7016000000 \\
\hline $\mathrm{H}$ & -5.5513000000 & -0.1134000000 & -2.0942000000 \\
\hline $\mathrm{H}$ & -8.0089000000 & -0.1111000000 & -2.0250000000 \\
\hline $\mathrm{C}$ & 0.6660000000 & -4.0743000000 & -3.1698000000 \\
\hline $\mathrm{H}$ & 1.3630000000 & -4.9028000000 & -3.2198000000 \\
\hline $\mathrm{H}$ & 1.1917000000 & -3.1577000000 & -2.880800000 \\
\hline $\mathrm{H}$ & -0.1056000000 & -4.2855000000 & -2.429500000 \\
\hline $\mathrm{H}$ & 0.2012000000 & -3.9250000000 & -4.138400000 \\
\hline
\end{tabular}

Compound ID : B2-11m

Calculation : OPT + FREQ, Basis Set : TZVP, Functional : BP86 


\begin{tabular}{|c|c|c|c|}
\hline \multicolumn{4}{|c|}{$\begin{array}{l}\text { Imaginary Frequency }=0 \\
\text { Dipole Moment }=5.75 \text { debye } \\
E=-0.190721 \text { hatree }\end{array}$} \\
\hline & 4.4242000000 & 7.9527000000 & -0.1893000000 \\
\hline & 3.8594000000 & 6.5803000000 & -0.1304000000 \\
\hline & 4.5538000000 & 5.5605000000 & 0.5225000000 \\
\hline & 4.0345000000 & 4.2748000000 & 0.5713000000 \\
\hline & 2.8040000000 & 3.9930000000 & -0.0394000000 \\
\hline $\mathrm{N}$ & 2.3226000000 & 2.6356000000 & -0.0281000000 \\
\hline & 1.0023000000 & 2.2541000000 & 0.3008000000 \\
\hline $\mathrm{O}$ & 0.0911000000 & 3.0715000000 & 0.3209000000 \\
\hline $\mathrm{C}$ & 0.7536000000 & 0.7768000000 & 0.6220000000 \\
\hline $\mathrm{N}$ & & -0.15 & -0.4791000000 \\
\hline$P$ & 1.6533000000 & -1.785 & 00000 \\
\hline $\mathrm{C}$ & 1.5402000000 & -2.90530000 & 701000000 \\
\hline $\mathrm{C}$ & & -2.7085 & 44000000 \\
\hline $\mathrm{C}$ & 2.2149000000 & -3.5465000000 & -3.5997000000 \\
\hline $\mathrm{C}$ & 1.2881000000 & -4.5856000000 & -3.5848000000 \\
\hline $\mathrm{C}$ & 0.4853000000 & 000000 & 8000000 \\
\hline $\mathrm{C}$ & 0.6125000000 & -3.94 & 5000000 \\
\hline $\mathrm{C}$ & 3.4669000000 & -1.6014000000 & 0.5254000000 \\
\hline $\mathrm{C}$ & 3.8270000000 & -0.6030000000 & 1.4277000000 \\
\hline $\mathrm{C}$ & 5.1580000000 & -0.429 & 00000 \\
\hline $\mathrm{C}$ & 6.1401000000 & -1.264 & 00000 \\
\hline $\mathrm{C}$ & & -2.26 & 00000 \\
\hline $\mathrm{C}$ & 4.4524000000 & -2.43 & 0000 \\
\hline $\mathrm{C}$ & 000000 & -2.68 & 0000 \\
\hline $\mathrm{C}$ & 1.6829000000 & -3.5 & 00000 \\
\hline $\mathrm{C}$ & & & 0000 \\
\hline $\mathrm{C}$ & -0.0158000000 & -4.2395000000 & 0000 \\
\hline $\mathrm{C}$ & -0.9345000000 & -3.4286000000 & 000000 \\
\hline $\mathrm{C}$ & -0.5513000000 & -2.65 & 00000 \\
\hline $\mathrm{C}$ & -1.6349000000 & 95000000 & 00000 \\
\hline $\mathrm{O}$ & -2.2918000000 & -2.66 & 7000000 \\
\hline $\mathrm{C}$ & -3.0276000000 & -2.1668000000 & 45000000 \\
\hline $\mathrm{O}$ & -3.4343000000 & -3.1235000000 & -1.2413000000 \\
\hline $\mathrm{C}$ & -3.3321000000 & -0.7068000000 & -0.9764000000 \\
\hline $\mathrm{C}$ & -2.0856000000 & 0.1723000000 & -1.1393000000 \\
\hline $\mathrm{C}$ & -1.0192000000 & -0.4750000000 & -2.0098000000 \\
\hline $\mathrm{C}$ & 0.3569000000 & 0.1245000000 & -1.7494000000 \\
\hline $\mathrm{O}$ & 0.9433000000 & 0.7999000000 & -2.5656000000 \\
\hline $\mathrm{N}$ & -4.2742000000 & -0.1813000000 & 0.0487000000 \\
\hline $\mathrm{C}$ & -5.2824000000 & 0.7452000000 & -0.3701000000 \\
\hline $\mathrm{O}$ & -5.7133000000 & 0.8677000000 & -1.5034000000 \\
\hline $\mathrm{O}$ & -5.7139000000 & 1.4126000000 & 0.7301000000 \\
\hline $\mathrm{C}$ & -6.8498000000 & 2.3144000000 & 0.6828000000 \\
\hline
\end{tabular}




\begin{tabular}{|c|c|c|c|}
\hline & -6.5776000000 & 3.5207000000 & \\
\hline & -8.1321000000 & 1.5947000000 & 0.2636000000 \\
\hline & -6.9680000000 & 2.7749000000 & 2.1435000000 \\
\hline & 2.1091000000 & 5.0093000000 & -0.7042000000 \\
\hline & 2.6375000000 & 6.2938000000 & -0.7396000000 \\
\hline & 3.6362000000 & 8.7160000000 & -0.2366000000 \\
\hline & 5.0522000000 & 8.0704000000 & -1.0832000000 \\
\hline & 5.0496000000 & 8.1750000000 & 0.6854000000 \\
\hline & 5.5153000000 & & 5000000 \\
\hline & 4.6023000000 & 3.4889000000 & \\
\hline & 3.0241000000 & 000000 & 1000000 \\
\hline & -0.3103000000 & 5000000 & 4000000 \\
\hline & 1.3964000000 & 000000 & \\
\hline & 3.0652000000 & 3000000 & -2.5 \\
\hline & 2.8431000000 & 8000000 & -4.4 \\
\hline $\mathrm{H}$ & 3000000 & -5.2 & \\
\hline & -0.2516000000 & -5.5 & -2.4 \\
\hline & -0.02 & -4.0 & \\
\hline & 6000000 & & \\
\hline & 9000000 & & \\
\hline H & 7.1877000000 & & \\
\hline & 6.5549000000 & & \\
\hline $\mathrm{H}$ & 74000000 & -3.2 & -0 . \\
\hline & 2.7259000000 & 0000000 & \\
\hline $\mathrm{H}$ & 2.03 & -4. & \\
\hline & 2000000 & -4.8 & \\
\hline $\mathrm{H}$ & -1.9 & -3. & \\
\hline & 0000000 & -1.5 & \\
\hline & & -0.8 & \\
\hline & -3.870400000 & -0.73 & \\
\hline $\mathrm{H}$ & -1.6606000000 & & \\
\hline & 0000000 & & 81400000 \\
\hline & 82000000 & 3000000 & \\
\hline H & -0.96 & -1.5 & -1.8 \\
\hline & -3.81 & & \\
\hline $\mathrm{H}$ & 6000000 & 3.9979000000 & 0.0236000000 \\
\hline & -6.55 & 3.2494000000 & \\
\hline & -7.36 & 4.2749000000 & -0.0860000000 \\
\hline $\mathrm{H}$ & -8.1184000000 & 1.3054000000 & -0.795800000 \\
\hline $\mathrm{H}$ & 95000000 & 3000000 & 0.411900000 \\
\hline . & -8.2989000000 & & 0.856600000 \\
\hline & -7.8048000000 & & \\
\hline $\mathrm{H}$ & -7.1509000000 & 1.9327000000 & 2.823900000 \\
\hline & -6.0589000000 & 3.2875000000 & \\
\hline $\mathrm{H}$ & 1.1481000000 & 4.8072000000 & -1.193100000 \\
\hline $\mathbf{H}$ & 2.0878000000 & 7.0897000000 & -1.255300000 \\
\hline
\end{tabular}


Compound ID : $\mathbf{6 d b} \mathbf{d}-\mathbf{7 m}$

Calculation : OPT + FREQ, Basis Set : TZVP, Functional : BP86

Imaginary Frequency $=0$

Dipole Moment $=2.75$ debye

$\mathrm{EE}=-0.345165$ hatree

$\begin{array}{lrrr}\mathrm{C} & 0.1399000000 & -6.1802000000 & -1.9614000000 \\ \mathrm{C} & 1.1991000000 & -5.2333000000 & -1.3959000000 \\ \mathrm{O} & 0.6011000000 & -4.0774000000 & -0.7515000000 \\ \mathrm{C} & -0.1686000000 & -4.1987000000 & 0.3566000000 \\ \mathrm{~N} & -0.4986000000 & -2.9247000000 & 0.9287000000 \\ \mathrm{O} & -0.5025000000 & -5.2472000000 & 0.8841000000 \\ \mathrm{C} & -0.7655000000 & -1.7512000000 & 0.0518000000 \\ \mathrm{C} & -0.1917000000 & -0.4575000000 & 0.6549000000 \\ \mathrm{O} & -0.4271000000 & 0.6365000000 & 0.1566000000 \\ \mathrm{~N} & 0.5938000000 & -0.5170000000 & 1.8371000000 \\ \mathrm{C} & 1.3262000000 & 0.6790000000 & 2.2933000000 \\ \mathrm{C} & 2.6441000000 & 0.9114000000 & 1.5547000000 \\ \mathrm{~N} & 3.0890000000 & 2.2636000000 & 1.4798000000 \\ \mathrm{O} & 3.3299000000 & -0.0022000000 & 1.1217000000 \\ \mathrm{C} & 4.1805000000 & 2.6741000000 & 0.6255000000 \\ \mathrm{C} & 5.4468000000 & 2.0958000000 & 0.7613000000 \\ \mathrm{C} & 6.4998000000 & 2.5562000000 & -0.0194000000 \\ \mathrm{C} & 6.3096000000 & 3.5957000000 & -0.9305000000 \\ \mathrm{C} & 5.0444000000 & 4.1716000000 & -1.0584000000 \\ \mathrm{C} & 3.9826000000 & 3.7169000000 & -0.2888000000 \\ \mathrm{C} & 7.4451000000 & 4.0941000000 & -1.7481000000 \\ \mathrm{C} & -2.2584000000 & -1.6066000000 & -0.2942000000 \\ \mathrm{C} & 2.1584000000 & -5.9742000000 & -0.4641000000 \\ \mathrm{C} & 1.9882000000 & -4.5911000000 & -2.5470000000 \\ \mathrm{C} & -3.2160000000 & -1.6109000000 & 0.8916000000 \\ \mathrm{C} & -3.4601000000 & -0.2315000000 & 1.4680000000 \\ \mathrm{O} & -3.1153000000 & 0.1767000000 & 2.5596000000 \\ \mathrm{O} & -4.1820000000 & 0.5734000000 & 0.6400000000 \\ \mathrm{C} & -4.3983000000 & 1.9325000000 & 1.0350000000 \\ \mathrm{C} & -5.0508000000 & 2.6081000000 & -0.1353000000 \\ \mathrm{C} & -6.4296000000 & 2.8191000000 & -0.1444000000 \\ \mathrm{C} & -7.0273000000 & 3.4619000000 & -1.2230000000 \\ \mathrm{C} & -6.2532000000 & 3.8917000000 & -2.2956000000 \\ \mathrm{C} & -4.8784000000 & 3.6792000000 & -2.2898000000 \\ \mathrm{H} & -4.2756000000 & 3.0394000000 & -1.2125000000 \\ & -0.3979000000 & -6.7217000000 & -1.1718000000 \\ \mathrm{H} & -0.6122000000 & -6.9311000000 & -2.6086000000 \\ \mathrm{H} & -1.2011000000 & -3.0028000000 & 1.6440000000\end{array}$




$\begin{array}{lrrr}\mathrm{H} & -0.2089000000 & -1.8811000000 & -0.9133000000 \\ \mathrm{H} & 1.1141000000 & -1.3606000000 & 1.9544000000 \\ \mathrm{H} & 1.5445000000 & 0.5315000000 & 3.3714000000 \\ \mathrm{H} & 0.6492000000 & 1.5565000000 & 2.2277000000 \\ \mathrm{H} & 2.3423000000 & 2.9252000000 & 1.4855000000 \\ \mathrm{H} & 5.6153000000 & 1.2735000000 & 1.4668000000 \\ \mathrm{H} & 4.8879000000 & 2.0972000000 & 0.0827000000 \\ \mathrm{H} & 2.9973000000 & 4.1845000000 & -0.4069000000 \\ \mathrm{H} & 8.1809000000 & 3.3042000000 & -1.9488000000 \\ \mathrm{H} & 7.9669000000 & 4.9064000000 & -1.2235000000 \\ \mathrm{H} & 7.1088000000 & 4.4884000000 & -2.7162000000 \\ \mathrm{H} & -2.5374000000 & -2.4426000000 & -0.9687000000 \\ \mathrm{H} & -2.3988000000 & -0.6859000000 & -0.8976000000 \\ \mathrm{H} & 1.6303000000 & -6.5087000000 & 0.3368000000 \\ \mathrm{H} & 2.8754000000 & -5.2888000000 & 0.0066000000 \\ \mathrm{H} & 2.7339000000 & -6.7189000000 & -1.0297000000 \\ \mathrm{H} & 1.3331000000 & -4.0375000000 & -3.2329000000 \\ \mathrm{H} & 2.5025000000 & -5.3643000000 & -3.1326000000 \\ \mathrm{H} & 2.7512000000 & -3.8919000000 & -2.1791000000 \\ \mathrm{H} & -2.8495000000 & -2.2814000000 & 1.7013000000 \\ \mathrm{H} & -4.1912000000 & -2.0331000000 & 0.5746000000 \\ \mathrm{H} & -3.4359000000 & 2.4177000000 & 1.2888000000 \\ \mathrm{H} & -5.0411000000 & 1.9520000000 & 1.9357000000 \\ \mathrm{H} & -7.0421000000 & 2.4787000000 & 0.6984000000 \\ \mathrm{H} & -8.1094000000 & 3.6282000000 & -1.2273000000 \\ \mathrm{H} & -6.7257000000 & 4.3969000000 & -3.1442000000 \\ \mathrm{H} & -4.2686000000 & 4.0160000000 & -3.1345000000 \\ & -3.1924000000 & 2.8699000000 & -1.2073000000\end{array}$

Compound ID : 6db'-11m

Calculation : OPT + FREQ, Basis Set : TZVP, Functional : BP86

Imaginary Frequency $=0$

Dipole Moment $=2.16$ debye

$\mathrm{EE}=-0.327090$ hatree

$\begin{array}{lrrc}\mathrm{C} & 0.8400000000 & 6.6914000000 & 1.1172000000 \\ \mathrm{C} & -0.6424000000 & 6.4207000000 & 0.8555000000 \\ \mathrm{O} & -0.8375000000 & 5.2419000000 & 0.0334000000 \\ \mathrm{C} & -0.4747000000 & 4.0046000000 & 0.4556000000 \\ \mathrm{~N} & -0.9416000000 & 3.0533000000 & -0.5039000000 \\ \mathrm{O} & 0.0879000000 & 3.7392000000 & 1.5055000000 \\ \mathrm{C} & -0.3387000000 & 1.6935000000 & -0.4951000000 \\ \mathrm{C} & 0.9722000000 & 1.6470000000 & -1.2929000000 \\ \mathrm{O} & 1.3013000000 & 2.3984000000 & -2.1906000000 \\ \mathrm{~N} & -4.3200000000 & -0.1387000000 & -1.8345000000\end{array}$




\begin{tabular}{|c|c|c|c|}
\hline $\mathrm{C}$ & -5.4472000000 & -0.9842000000 & -2.2689000000 \\
\hline $\mathrm{C}$ & -6.6750000000 & -0.8834000000 & -1.3641000000 \\
\hline $\mathrm{N}$ & -7.5585000000 & -2.0011000000 & -1.3817000000 \\
\hline $\mathrm{O}$ & -6.9426000000 & 0.1220000000 & -0.7234000000 \\
\hline $\mathrm{C}$ & -8.6247000000 & -2.1603000000 & -0.4181000000 \\
\hline $\mathrm{C}$ & -9.6143000000 & -1.1810000000 & -0.2848000000 \\
\hline $\mathrm{C}$ & -10.6699000000 & -1.3864000000 & 0.5950000000 \\
\hline $\mathrm{C}$ & -10.7566000000 & -2.5623000000 & 1.3410000000 \\
\hline $\mathrm{C}$ & -9.7702000000 & -3.5396000000 & 1.1968000000 \\
\hline $\mathrm{C}$ & -8.7075000000 & -3.3443000000 & 0.3258000000 \\
\hline $\mathrm{C}$ & -11.8805000000 & -2.7674000000 & 2.2902000000 \\
\hline $\mathrm{C}$ & -1.3230000000 & 0.6873000000 & -1.1114000000 \\
\hline $\mathrm{C}$ & -1.4245000000 & 6.3380000000 & 2.1671000000 \\
\hline $\mathrm{C}$ & -1.2242000000 & 7.5380000000 & -0.0239000000 \\
\hline $\mathrm{C}$ & -2.5031000000 & 0.4234000000 & -0.1875000000 \\
\hline $\mathrm{C}$ & -3.4416000000 & -0.5997000000 & -0.8016000000 \\
\hline $\mathrm{O}$ & -3.4315000000 & -1.7856000000 & -0.5109000000 \\
\hline $\mathrm{O}$ & 1.7781000000 & 0.6235000000 & -0.9050000000 \\
\hline $\mathrm{C}$ & 3.0280000000 & 0.3896000000 & -1.5527000000 \\
\hline $\mathrm{C}$ & 4.1829000000 & 0.8758000000 & -0.7153000000 \\
\hline $\mathrm{C}$ & 5.1959000000 & 0.0106000000 & -0.2854000000 \\
\hline $\mathrm{C}$ & 6.2319000000 & 0.5416000000 & 0.4799000000 \\
\hline $\mathrm{C}$ & 6.2830000000 & 1.8896000000 & 0.8132000000 \\
\hline $\mathrm{C}$ & 5.2807000000 & 2.7435000000 & 0.3688000000 \\
\hline $\mathrm{C}$ & 4.2382000000 & 2.2359000000 & -0.3948000000 \\
\hline $\mathrm{O}$ & 5.0819000000 & -2.0751000000 & -2.1472000000 \\
\hline $\mathrm{P}$ & 5.2774000000 & -1.7912000000 & -0.7091000000 \\
\hline $\mathrm{C}$ & 6.8857000000 & -2.3834000000 & -0.0766000000 \\
\hline $\mathrm{C}$ & 7.9230000000 & -2.5648000000 & -0.9869000000 \\
\hline $\mathrm{C}$ & 9.1617000000 & -3.0426000000 & -0.5675000000 \\
\hline $\mathrm{C}$ & 9.3714000000 & -3.3455000000 & 0.7733000000 \\
\hline $\mathrm{C}$ & 8.3396000000 & -3.1718000000 & 1.6905000000 \\
\hline $\mathrm{C}$ & 7.1055000000 & -2.6939000000 & 1.2625000000 \\
\hline $\mathrm{C}$ & 4.0498000000 & -2.5599000000 & 0.3913000000 \\
\hline $\mathrm{C}$ & 3.7371000000 & -2.0371000000 & 1.6427000000 \\
\hline $\mathrm{C}$ & 2.7937000000 & -2.6554000000 & 2.4568000000 \\
\hline $\mathrm{C}$ & 2.1513000000 & -3.8095000000 & 2.0196000000 \\
\hline $\mathrm{C}$ & 2.4541000000 & -4.3380000000 & 0.7694000000 \\
\hline $\mathrm{C}$ & 3.3998000000 & -3.7129000000 & -0.0386000000 \\
\hline $\mathrm{H}$ & 1.2809000000 & 5.9574000000 & 1.8047000000 \\
\hline $\mathrm{H}$ & 0.9688000000 & 7.6829000000 & 1.5710000000 \\
\hline $\mathrm{H}$ & 1.4248000000 & 6.6737000000 & 0.1882000000 \\
\hline $\mathrm{H}$ & -1.0711000000 & 3.4093000000 & -1.4293000000 \\
\hline $\mathrm{H}$ & -0.1333000000 & 1.4112000000 & 0.5710000000 \\
\hline $\mathrm{H}$ & -4.5740000000 & 0.8218000000 & -1.7353000000 \\
\hline $\mathrm{H}$ & -5.0887000000 & -2.0298000000 & -2.3693000000 \\
\hline
\end{tabular}




$\begin{array}{cccc}\mathrm{H} & -5.7289000000 & -0.6476000000 & -3.2881000000 \\ \mathrm{H} & -7.1006000000 & -2.8633000000 & -1.5871000000 \\ \mathrm{H} & -9.5601000000 & -0.2468000000 & -0.8563000000 \\ \mathrm{H} & -11.4426000000 & -0.6164000000 & 0.7022000000 \\ \mathrm{H} & -9.8341000000 & -4.4671000000 & 1.7772000000 \\ \mathrm{H} & -7.9437000000 & -4.1260000000 & 0.2299000000 \\ \mathrm{H} & -12.7897000000 & -2.2441000000 & 1.9656000000 \\ \mathrm{H} & -11.6154000000 & -2.3834000000 & 3.2850000000 \\ \mathrm{H} & -12.1313000000 & -3.8306000000 & 2.4025000000 \\ \mathrm{H} & -0.7887000000 & -0.2650000000 & -1.3073000000 \\ \mathrm{H} & -1.6765000000 & 1.0432000000 & -2.1011000000 \\ \mathrm{H} & -1.0000000000 & 5.5971000000 & 2.8576000000 \\ \mathrm{H} & -2.4758000000 & 6.0720000000 & 1.9955000000 \\ \mathrm{H} & -1.4078000000 & 7.3092000000 & 2.6790000000 \\ \mathrm{H} & -0.6950000000 & 7.6203000000 & -0.9826000000 \\ \mathrm{H} & -1.1386000000 & 8.5065000000 & 0.4858000000 \\ \mathrm{H} & -2.2872000000 & 7.3725000000 & -0.2442000000 \\ \mathrm{H} & -3.0480000000 & 1.3632000000 & 0.0371000000 \\ \mathrm{H} & -2.1425000000 & 0.0489000000 & 0.7928000000 \\ \mathrm{H} & 3.0850000000 & 0.8228000000 & -2.5706000000 \\ \mathrm{H} & 3.0173000000 & -0.7140000000 & -1.6422000000 \\ \mathrm{H} & 7.0346000000 & -0.1228000000 & 0.8312000000 \\ \mathrm{H} & 7.1096000000 & 2.2762000000 & 1.4181000000 \\ \mathrm{H} & 5.3116000000 & 3.8094000000 & 0.6172000000 \\ \mathrm{H} & 3.4531000000 & 2.9123000000 & -0.7537000000 \\ \mathrm{H} & 7.7572000000 & -2.3373000000 & -2.0486000000 \\ \mathrm{H} & 9.9689000000 & -3.1821000000 & -1.2941000000 \\ \mathrm{H} & 10.3440000000 & -3.7222000000 & 1.1063000000 \\ \mathrm{H} & 8.4977000000 & -3.4135000000 & 2.7466000000 \\ \mathrm{H} & 6.2884000000 & -2.5671000000 & 1.9863000000 \\ \mathrm{H} & 4.2312000000 & -1.1166000000 & 1.9817000000 \\ \mathrm{H} & 2.5535000000 & -2.2315000000 & 3.4373000000 \\ \mathrm{H} & 1.4060000000 & -4.2975000000 & 2.6565000000 \\ & 1.9466000000 & -5.2428000000 & 0.4190000000 \\ & 3.6303000000 & -4.1268000000 & -1.0292000000\end{array}$

Table 2.

\begin{tabular}{lllll} 
dG $(\mathrm{kcal} / \mathrm{mol})$ & dG (Hatree) & dH & dH(Hatree) & Zero-point E \\
\hline
\end{tabular}




\begin{tabular}{cccccc}
\hline Entry & & & (kcal/mol) & & $\begin{array}{c}\text { correction } \\
\text { (Hatree) }\end{array}$ \\
\hline $\mathbf{5 b}$ & 254.73 & 0.405944 & 291.58 & 0.46466 & 0.18372 \\
$\mathbf{4 d}$ & 628.22 & 1.001126 & 711.69 & 1.13415 & 0.658 \\
& & & $\mathbf{1 0 0 3 . 2 7}$ & $\mathbf{1 . 5 9 8 8 1}$ & \\
$\mathbf{4 d 5 b}$ & 824.52 & 1.313948 & 929.11 & 1.48063 & 0.83405 \\
& & & & & \\
$\mathbf{A 1 - 7 m - T S}$ & 825.67 & 1.315794 & 930.96 & 1.48358 & 0.83447 \\
$\mathbf{B 1 - 1 1 m - T S}$ & 833.93 & 1.328944 & 932.24 & 1.48561 & 0.83662 \\
& & & & & \\
$\mathbf{A 2 - 7 m}$ & 698.79 & 1.113590 & 800.28 & 1.275325 & 0.752906 \\
$\mathbf{B 2 - 1 1 m}$ & 718.74 & 1.145384 & 804.49 & 1.28204 & 0.71232 \\
& & & & & \\
$\mathbf{6 d b - 7 m}$ & 425.74 & 0.678463 & 502.01 & 0.8 & 0.55363 \\
$\mathbf{6 d b} \mathbf{- 1 1 m}$ & 641.93 & 1.022983 & 739.11 & 1.17785 & 0.72676 \\
\hline
\end{tabular}

